DOC.20041012.0002

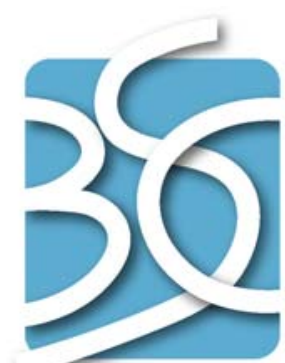

BECHTEL SAIC COMPANYLC
QA: QA

ANL-NBS-HS-000034 REV 02

October 2004

NOTICE OF OPEN CHANGED DOCUMENTS - THIS DOCUMENT IS IMPACTED BY THE LISTED CHANGE DOCUMENTS AND CANNOT BE USED WITHOUT THEM.

1) ACN-001, DATED 02/10/2005

\title{
Water-Level Data Analysis for the Saturated Zone Site-Scale Flow and Transport Model
}

Prepared for:

U.S. Department of Energy

Office of Civilian Radioactive Waste Management

Office of Repository Development

1551 Hillshire Drive

Las Vegas, Nevada 89134-6321

Prepared by:

Bechtel SAIC Company, LLC

1180 Town Center Drive

Las Vegas, Nevada 89144

Under Contract Number

DE-AC28-01RW12101 


\section{DISCLAIMER}

This report was prepared as an account of work sponsored by an agency of the United States Government. Neither the United States Government nor any agency thereof, nor any of their employees, nor any of their contractors, subcontractors or their employees, makes any warranty, express or implied, or assumes any legal liability or responsibility for the accuracy, completeness, or any third party's use or the results of such use of any information, apparatus, product, or process disclosed, or represents that its use would not infringe privately owned rights. Reference herein to any specific commercial product, process, or service by trade name, trademark, manufacturer, or otherwise, does not necessarily constitute or imply its endorsement, recommendation, or favoring by the United States Government or any agency thereof or its contractors or subcontractors. The views and opinions of authors expressed herein do not necessarily state or reflect those of the United States Government or any agency thereof. 
QA: QA

Water-Level Data Analysis for the Saturated Zone Site-Scale Flow and Transport Model

ANL-NBS-HS-000034 REV 02

October 2004 


\section{OCRWM}

\section{Scientific Analysis Signature Page/ Change History}

\section{Scientific Anglysls Title}

Water-Level Dara Analysis for the Saturated Zone Sitc-Scale Flow and Transport Model

3. Dl (including Revision Number)

ANL-NBS-HS-000034 REV 02

\begin{tabular}{l|l}
\hline $\begin{array}{l}\text { 4. Total Appendices } \\
\text { Three (3) }\end{array}$ & $\begin{array}{l}\text { 5. Number of Pages in Each Appondix } \\
\text { A-38, B-12. C-10 }\end{array}$ \\
\hline
\end{tabular}

Three (3)

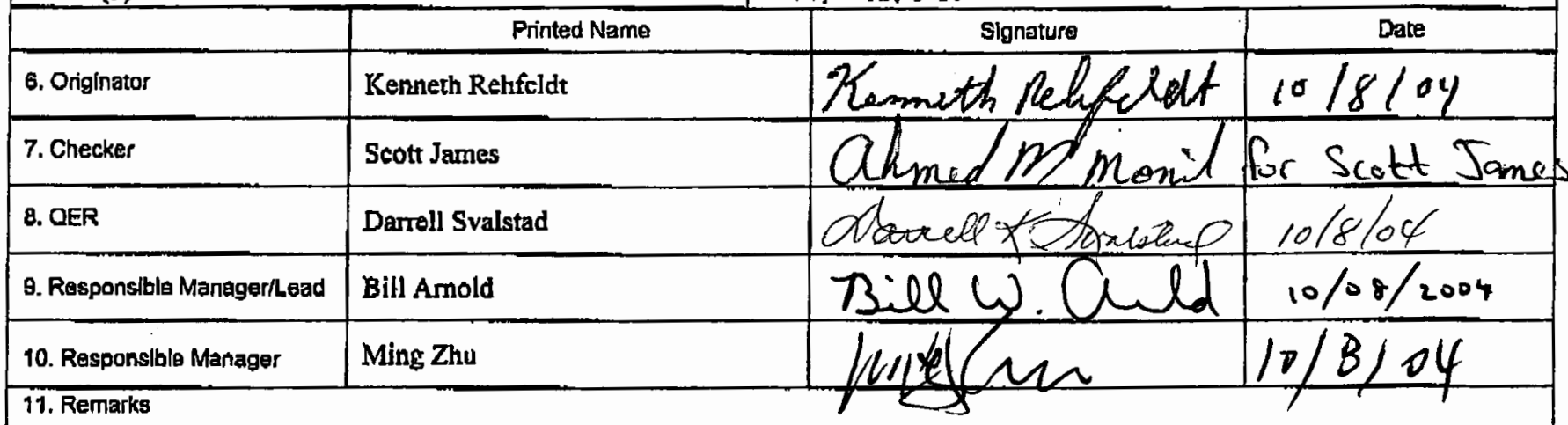

Errata to Rev 01 identified in DC Tracking numbers 33545 and 38658 have been incorporated in Rev 02.

\begin{tabular}{|c|c|}
\hline & Change History \\
\hline 12. Revision No. & 13. Description of Change \\
\hline REV 00 & Initial issue \\
\hline REV 00/1CN 01 & $\begin{array}{l}\text { ICN to replace record accessioned as number MOL.20000609.0111 with Data Tracking Numbers and } \\
\text { reviso assumption text concerning this matter (Section 5.3). Update Section } 8.2 \text { to reflect current } \\
\text { procedures, and update Sections } 8.1 \text { and } 8.4 \text { to reflect current DIRS references. }\end{array}$ \\
\hline REV 01 & $\begin{array}{l}\text { This revision updates the water-level data with dara from Nye Counry Early Warning Drilling Program } \\
\text { wells and Well USW WT-24. This report also includes a discussion of vertical bead differences in the } \\
\text { Yucca Mountain area, and a poientiometric-surface map that represents an altemate conceptual model } \\
\text { from that presented in Rev00/1CN } 01 \text { of water levels north of Yucea Mountain. Additional text } \\
\text { changes were made for the purpose of clarification or editorial correction. } \\
\text { Note: Changes were too extensive to denote by a black vertical line in the margins. }\end{array}$ \\
\hline
\end{tabular}


REV 02

This revision builds upon REV 01 to add enhanced discussion of uncertainty in the potentiometricsurface map. New water-level data was examined and the anticipated impacts to the potentiometric surface are noted. However, no changes were made to the map provided to the flow model for calibration. The map and analysis were not updated with more recent data because it was shown that updating would not make significant changes to the map or the calibration targets. Errata as identified in CR 1667 (DC Tracking Number 38658, DOC.20040303.0006) and TER-02-0087 (DC Tracking Number 33545, MOL.20020917.0136) were incorporated. The revision adds four new sections, Section 4-2 addresses the Yucca Mountain Review Criteria, Section 6-1 provides objectives of the analysis, Section 6-2 addressing the Features, Events, and Processes (FEPs), and Section 7.2 describes the incorporation of the YMRP acceptance criteria. The potentiometric-surface map and mean waterlevel altitude remain the same as USGS (2004 [DIRS 168473]). Corrected outputs were supplied to output DTNs GS010908312332.002 and GS010908312332.003. Two entries were corrected, one in Table A-2 for the number of data points for Well NC-EWDP-3S probe 2 and the other to Table 6-4 for the vertical hydraulic head difference for Wells NC-EWDP-2D and NE-EWDP-2DB. The entire scientific analysis documentation was revised. Changes were too extensive to use Step 5.6e1) per APSIII.9Q/Rev.1/ICN 7. 


\section{CONTENTS}

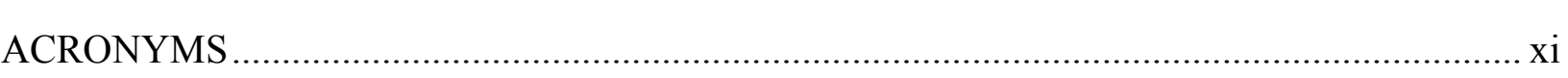

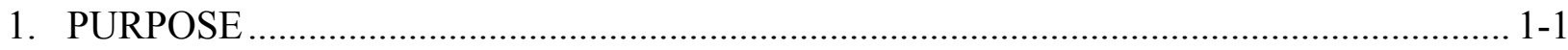

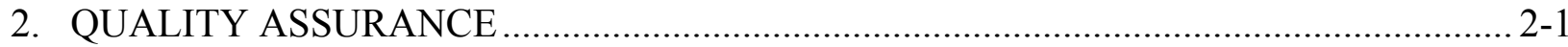

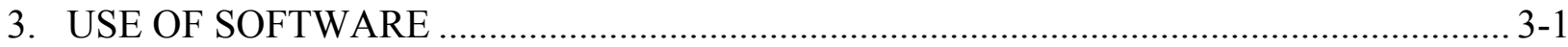

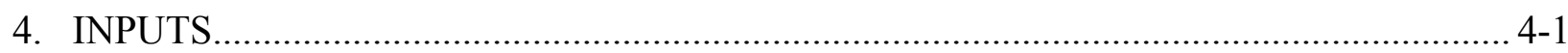

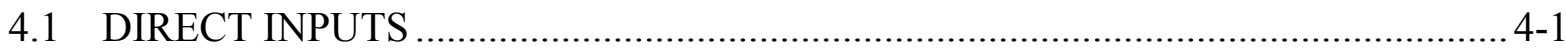

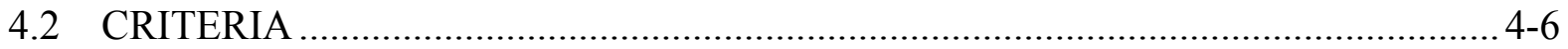

4.3 CODES, STANDARDS, AND REGULATIONS .................................................. 4-7

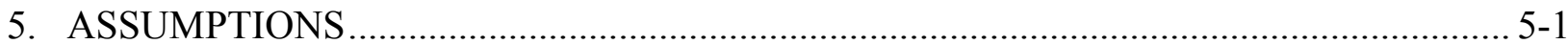

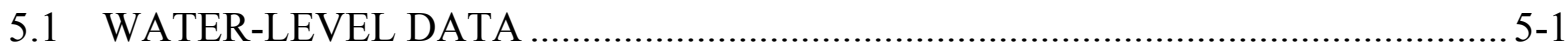

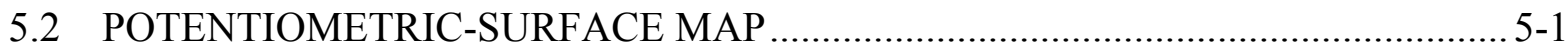

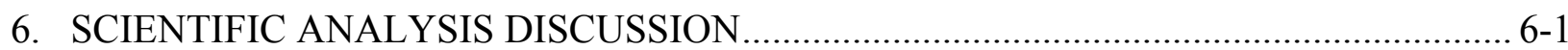

6.1 OBJECTIVES OF THE ANALYSIS .................................................................

6.2 FEATURES, EVENTS, AND PROCESSES FOR THIS ANALYSIS REPORT ....... 6-1

6.2.1 Scientific Analysis Assumptions ........................................................... 6-2

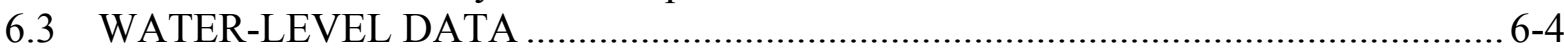

6.3.1 Qualification of Unqualified Data ................................................................. 6-9

6.3.2 Vertical Head Differences............................................................................. 6-9

6.3.3 Key Technical Issues (KTI) Related to Potentiometric Surface and Vertical

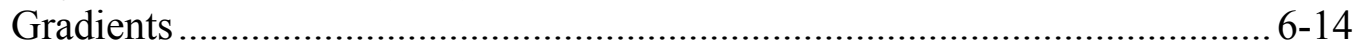

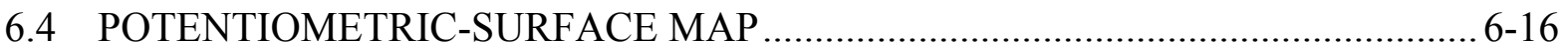

6.5 UNCERTAINTY IN THE POTENTIOMETRIC-SURFACE ELEVATION MAP.. 6-22

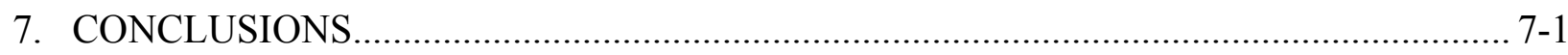

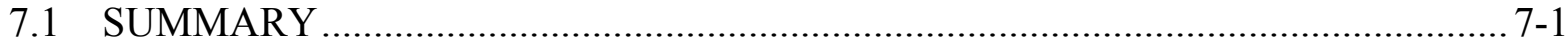

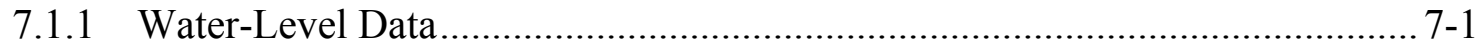

7.1.2 Potentiometric-Surface Map ……............................................................

7.2 INCORPORATION OF THE YMRP ACCEPTANCE CRITERIA ............................ 7-3

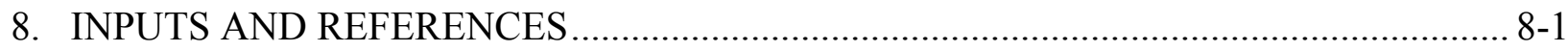

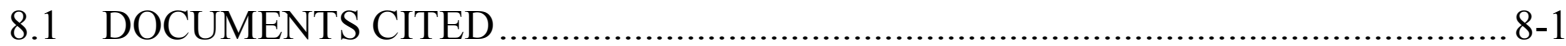

8.2 STANDARDS, REGULATIONS, AND PROCEDURES ………………................ 8-5

8.3 SOURCE DATA, LISTED BY DATA TRACKING NUMBER …............................ 8-5

8.4 OUTPUT DATA, LISTED BY DATA TRACKING NUMBER ……...................... 8-9

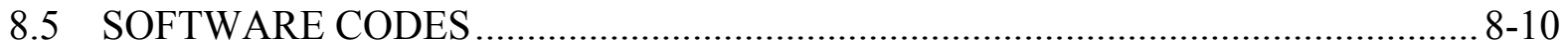

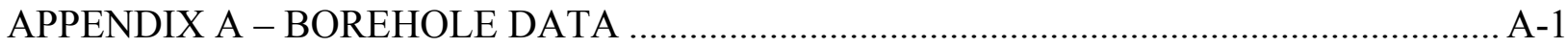

APPENDIX B - QUALIFICATION OF WATER-LEVEL DATA........................................ B-1

APPENDIX C - DATA QUALIFICATION PLANS ……………………............................ C-1 


\section{INTENTIONALLY LEFT BLANK}




\section{FIGURES}

Page

1-1. Location Map of the Study Area and Associated Geographic Features .......................... 1-2

1-2. Location of Boreholes Used to Characterize the Potentiometric Surface in the Yucca

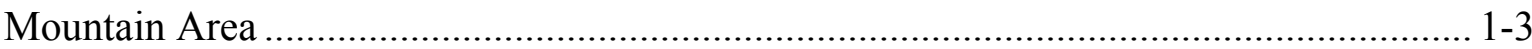

1-3. Generalized Flow of Information Among Reports Pertaining to Flow and Transport in the SZ $1-6$

6-1. 2001 Potentiometric-Surface Map, Assuming Perched Conditions North of Yucca Mountain, in the SZ Site-Scale Flow and Transport Model Area ................................. 6-18

6-2. Revised Potentiometric-Surface Map Showing Possible Changes After Including EWDP Phases III and IV Wells

6-3. 2000 Potentiometric Surface used in the SZ Site-Scale Flow Model........................... 6-38

6-4. Plan-View Schematic Showing Boreholes, Contoured Water Table (Elevations in Meters), UZ Model Boundary, Repository Outline, ESF, and ECRB Cross-Drift. 


\section{INTENTIONALLY LEFT BLANK}




\section{TABLES}

Page

3-1. Software Used to Support Analysis Activity .......................................................... 3-1

4-1. Direct Input Data Sources ....................................................................................... 4-4

4-2. Project Requirements Addressed in This Report ............................................................ 4-7

6-1. Features, Events, and Processes Included in TSPA-LA and Relevant to This Report ...... 6-2

6-2. Indirect Input Data Sources ................................................................................... 6-5

6-3. Comparison of Water-Level Values through 1995 to Average Values in 1999 and

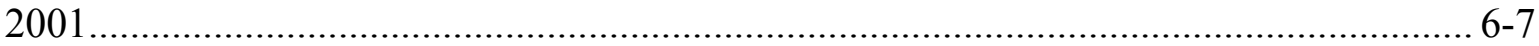

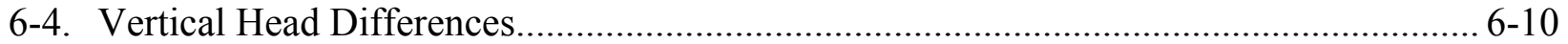

6-5. Water Levels for the Time Period of January 2003 to August 2003 Used for Assessment of New Data ............................................................................................ 6-27

6-5. Water Levels for the Time Period of January 2003 to August 2003 Used for Assessment of New Data (Continued) ....................................................................... 6-28

6-6. Summary of Potential Uncertainties Impacting the Potentiometric-Surface Map........... 6-34 


\section{INTENTIONALLY LEFT BLANK}




\section{ACRONYMS}

ACM alternative conceptual model

AIN

additional information needed

DOE

U.S. Department of Energy

DTN

data tracking number

EWDP Early Warning Drilling Program

GIS Geographic Information System

HFM hydrogeologic framework model

ICN interim change notice

ID identification

IRAN impact review action notice

LHG large hydraulic gradient

NRC U.S. Nuclear Regulatory Commission

NWIS National Water Information System

OCRWM Office of Civilian Radioactive Waste Management

ORD Office of Repository Development

PI principal investigator

QA quality assurance

QARD Quality Assurance Requirements and Description

SC safety category

SZ saturated zone

TDMS Technical Data Management System

TSPA total system performance assessment

TWP technical work plan

USFIC unsaturated and saturated zone flow under isothermal conditions

USGS United States Geological Survey

UTM Universal Transverse Mercator

UZ unsaturated zone

YMP Yucca Mountain Project 


\section{INTENTIONALLY LEFT BLANK}




\section{PURPOSE}

This report is an updated analysis of water-level data performed to provide the Saturated Zone Site-Scale Flow Model (BSC 2004 [DIRS 170037]) (referred to as the saturated zone (SZ) site-scale flow model or site-scale SZ flow model in this report) with the configuration of the potentiometric surface, target water-level data, and hydraulic gradients for calibration of groundwater flow models. This report also contains an expanded discussion of uncertainty in the potentiometric-surface map. The analysis of the potentiometric data presented in Revision 00 of this report (USGS 2001 [DIRS 154625]) provides the configuration of the potentiometric surface, target heads, and hydraulic gradients for the calibration of the SZ site-scale flow model (BSC 2004 [DIRS 170037]). Revision 01 of this report (USGS 2004 [DIRS 168473]) used updated water-level data for selected wells through the year 2000 as the basis for estimating water-level altitudes and the potentiometric surface in the SZ site-scale flow and transport model domain based on an alternative interpretation of perched water conditions. That revision developed computer files containing:

- Water-level data within the model area (DTN: GS010908312332.002)

- A table of known vertical head differences (DTN: GS010908312332.003)

- A potentiometric-surface map (DTN: GS010608312332.001) using an alternative concept from that presented by USGS (2001 [DIRS 154625]) for the area north of Yucca Mountain.

The updated water-level data presented in USGS (2004 [DIRS 168473]) include data obtained from the Nye County Early Warning Drilling Program (EWDP) Phases I and II and data from Borehole USW WT-24. This document is based on Revision 01 (USGS 2004 [DIRS 168473]) and expands the discussion of uncertainty in the potentiometric-surface map. This uncertainty assessment includes an analysis of the impact of more recent water-level data and the impact of adding data from the EWDP Phases III and IV wells.

In addition to being utilized by the SZ site-scale flow model, the water-level data and potentiometric-surface map contained within this report will be available to other government agencies and water users for groundwater management purposes. The potentiometric surface defines an upper boundary of the site-scale flow model and provides information useful to estimation of the magnitude and direction of lateral groundwater flow within the flow system. Therefore, the analysis documented in this revision is important to SZ flow and transport calculations in support of total system performance assessment (TSPA).

The source data associated with this analysis include water-level data from boreholes within, and from one borehole (UE-25 J-11) adjacent to, the SZ site-scale flow and transport model area. The SZ site-scale flow and transport model area (Figure 1-1) is between a Universal Transverse Mercator (UTM) Easting of 533,340 $\mathrm{m}$ and 563,340 $\mathrm{m}$ and a UTM Northing of 4,046,782 $\mathrm{m}$ and 4,091,782 m (Zone 11, North American Datum 1927). The following types of information were gathered: Borehole site name/identification (ID), location, land-surface altitude, water-level altitude, data source, reliability of data, minimum and maximum water levels (range), and open 
interval monitored with the associated water-level altitude and type. Figure 1-2 is a map of the locations and names of the wells used in the creation of the potentiometric-surface map.

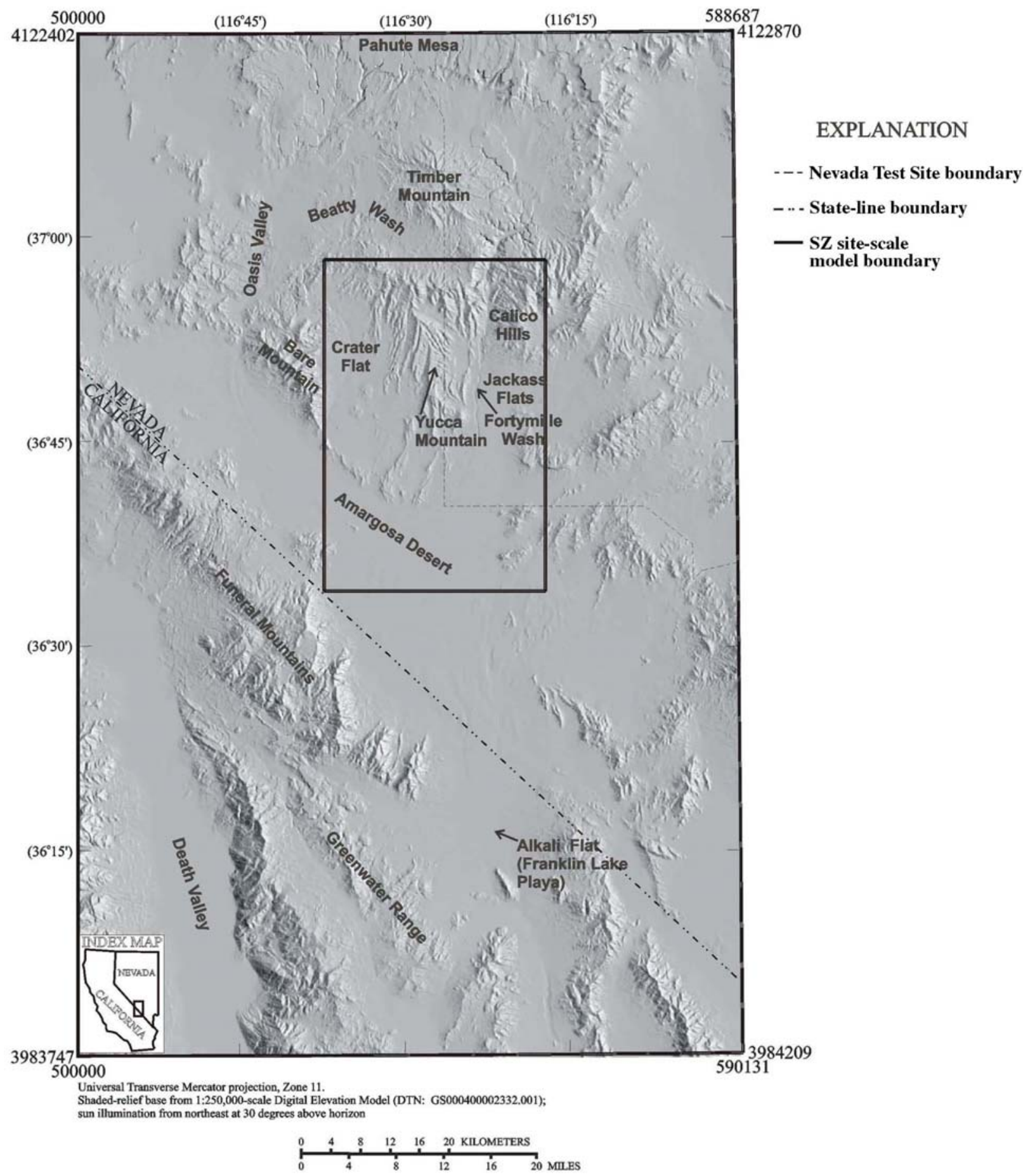

Source: USGS (2001 [DIRS 154625], Figure 1-1).

Figure 1-1. Location Map of the Study Area and Associated Geographic Features 


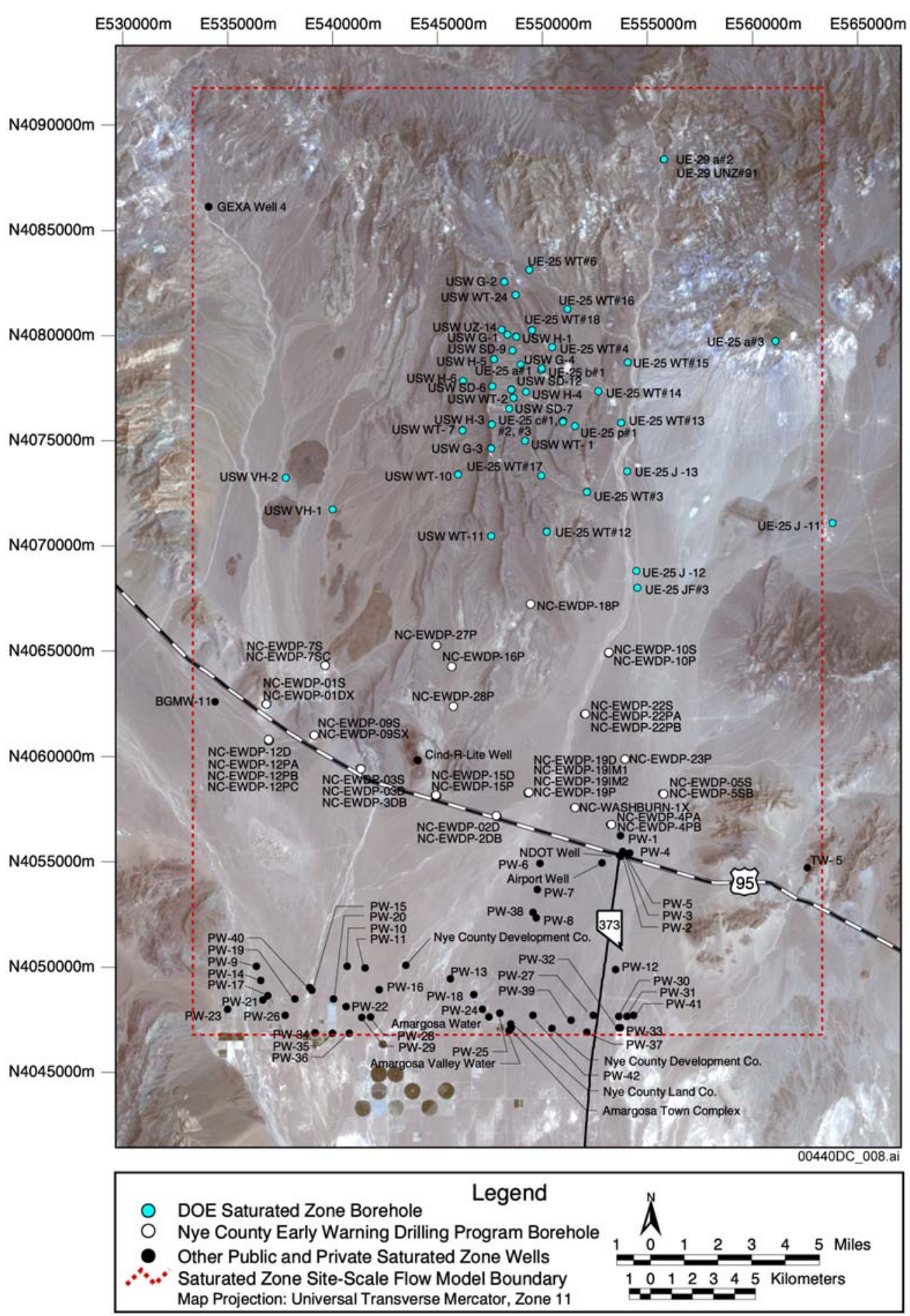

Sources: GS010608312331.001 (Output of this analysis), DTN: MO0103COV01031.000 [DIRS 155271], DTN: MO0107COV01057.000 [DIRS 157194], DTN: MO0203GSC02034.000 [DIRS 168375], DTN: MO0206GSC02074.000 [DIRS 168378], DTN: 0307GSC03094.000 [DIRS 170556].

Figure 1-2. Location of Boreholes Used to Characterize the Potentiometric Surface in the Yucca Mountain Area 
This report was conducted under Technical Work Plan for: Natural System - Saturated Zone Analysis and Model Report Integration (BSC 2004 [DIRS 171421]). The Technical Work Plan was prepared in accordance with AP-2.27Q, Planning for Science Activities. No deviations from the Technical Work Plan were necessary to complete this revision of this report.

The scope of the potentiometric-surface analysis includes:

- Compilation of water-level data within the SZ site-scale flow model area through 2003. Included are (1) data from the original analysis (USGS 2001 [DIRS 154625]) with end dates from 1952 to 1999 , depending on availability of data at the time, (2) data from the alternative interpretation conducted in 2001 (USGS 2004 [DIRS 168473]) with end dates in 2000 for the Nye County wells available at the time, (3) data from Yucca Mountain site wells through 2001 to assess temporal variability of water levels, and (4) data from the Nye County wells through 2003 to assess the impact of the new well locations.

- Removal of duplicate measurements and sites.

- Tabulation of measurement precision, where known.

- Assessment of the general reliability of the data.

- Tabulation of the range in water levels for use in uncertainty analyses.

- Documentation of the applicable use of water levels (potentiometric surface development and/or SZ site-scale flow model calibration).

- Generation of the potentiometric-surface map representative of the early 1990s (the time period of the regional-scale SZ flow model (D'Agnese et al. 1997 [DIRS 100131]) that is used to provide boundary conditions to the SZ site-scale flow model (BSC 2004 [DIRS 170037]).

- Generation of a table of known vertical head differences within the SZ site-scale flow model area. These head differences provide additional calibration targets for the model.

- The data used to create the potentiometric surface map is taken from USGS (2001 [DIRS 154625]) with end dates in 1995 or 1996. An assessment is made of the impact of more recent data in the vicinity of the Yucca Mountain repository collected in 1999, 2001, and 2003. The data for those wells is taken from USGS (2001 [DIRS 154625]) with end dates in 1995 or 1996 . This assessment is intended to demonstrate that the more recent data does not alter the interpretation using the data from USGS (2001 [DIRS 154625]).

- Assessment of water-level uncertainty, discussion of the impacts of the uncertainty on the potentiometric surface, and comparison of the potentiometric surface from this analysis with surfaces generated by other analyses. 
In this analysis, the water-level data are used to generate a single representative potentiometric surface (Figure 6-1) for the SZ site-scale flow and transport model domain. This revision of the potentiometric surface represents an alternative concept from that presented in Revision 00 of this report (USGS 2001 [DIRS 154625]) of the northern part of Yucca Mountain, which has been termed the "large hydraulic gradient (LHG) area" (Ervin et al. 1994 [DIRS 100633], p. 7). The current concept assumes that water levels in Boreholes USW G-2 and UE-25 WT \#6 represent perched conditions, and are not representative of the regional potentiometric surface. The regional potentiometric surface is thus located below the water levels measured in Boreholes USW G-2 and UE-25 WT\#6.

Recent geochemical analyses indicate that the perched water alternative hypothesis utilized in this document is consistent with observed geochemical parameters (Paces et al 2002 [DIRS 158817], pp. 760 to 762; BSC 2004 [DIRS 170037], Appendix A).

\section{Limitations}

The target water levels identified via this analysis are applicable to the time period in the early 1990s. Predevelopment water levels or expected water levels resulting from future climates are not addressed in this analysis. The potentiometric surface represents the top of the SZ only. The data were insufficient to define potentiometric surfaces deep within the SZ such as at the top of the carbonate aquifer or deep within the volcanic units.

\section{Role of the Potentiometric-Surface Analysis in SZ Flow and Transport}

Figure 1-3 shows the relationship of this report to other model reports that also pertain to flow and transport in the SZ. Figure 1-3 also shows the flow of key information among the $\mathrm{SZ}$ reports. It should be noted that Figure 1-3 does not contain a complete representation of the data and parameter inputs and outputs of all SZ reports, nor does it show inputs external to this suite of SZ reports. This water-level analysis is one of several analyses that provide input to the SZ site-scale flow model. The potentiometric-surface analysis provides the calibration target water levels for flow model calibration, an assessment of expected hydraulic gradients, and the configuration of the potentiometric surface that would be expected to be reproduced by the sitescale groundwater flow model. Specifically, the 2000 potentiometric surface described in USGS (2001 [DIRS 154625]) was used in the development of the SZ site-scale flow model and the 2001 potentiometric surface described in USGS (2004 [DIRS 168473]) and in this report is used in the evaluation of an alternative conceptual model of the SZ site-scale flow model. 

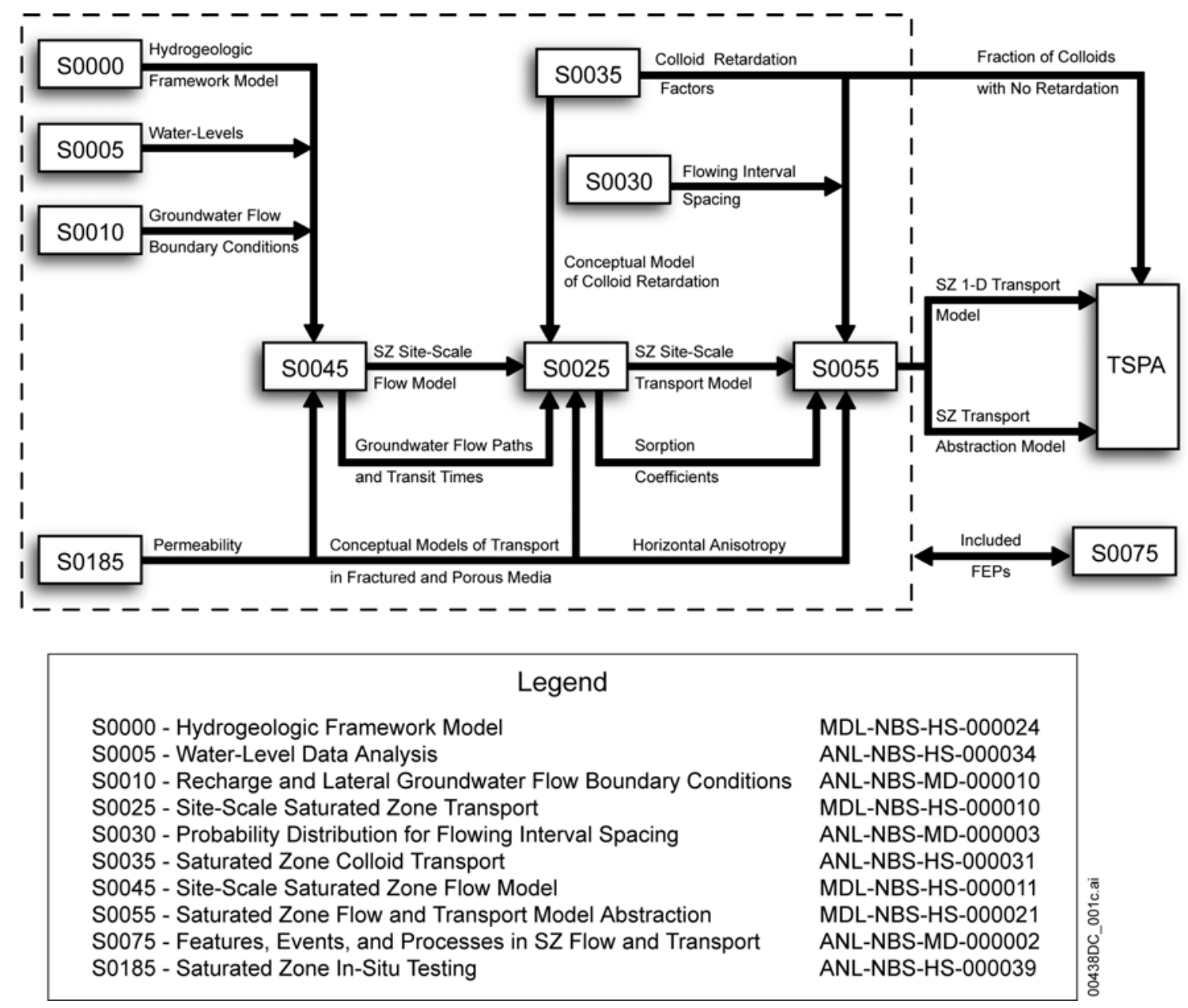

NOTE: This figure is a simplified representation of the flow of information among SZ reports. See the DIRS report of each report for a complete listing of data and parameter inputs. This figure does not show inputs external to this suite of SZ reports.

Figure 1-3. Generalized Flow of Information Among Reports Pertaining to Flow and Transport in the SZ 


\section{QUALITY ASSURANCE}

The scientific analyses documented in this report were evaluated in accordance with AP-2.27Q, Planning for Science Activities, and AP-SIII.9Q, Scientific Analyses, and were determined in the Technical Work Plan (TWP) (BSC 2004 [DIRS 171421], Section 8.1) to be quality-affecting and subject to the requirements of the U.S. Department of Energy (DOE), Office of Civilian Radioactive Waste Management (OCRWM) Quality Assurance Requirements and Description (DOE 2004 [DIRS 171539]). Accordingly, efforts to develop this report have been conducted in accordance with the Office of Repository Development (ORD) quality assurance (QA) program using approved procedures identified in the TWP for: Natural System - Saturated Zone Analysis Model Report Integration (BSC 2004 [DIRS 171421]). The work presented in this revision was documented in accordance with AP-SIII.9Q.

This analysis report provides a hydrologic property of a category of the natural barrier system (the rock and alluvial material below and down gradient from the repository) that is important to the demonstration of compliance with the post-closure performance objectives prescribed in 10 CFR 63.113. Therefore, it is classified on the Q-List (BSC 2004 [DIRS 168361]) as "SC" (Safety Category), reflecting its importance to waste isolation, as defined in AP-2.22Q, Classification Analyses and Maintenance of the Q-List. The report contributes to the analysis data used to support postclosure performance assessment; the conclusions do not directly impact preclosure engineering features important to safety, as defined in AP-2.22Q.

The majority of this analysis was performed as part of Revision 01 of this report (USGS 2004 [DIRS 168473]) and is documented in a scientific notebook (Tucci 2001 [DIRS 155410]). Methods to control electronically managed data are documented in a scientific notebook (Tucci 2001 [DIRS 155410], pp. 8 and 77). Work performed on this revision was in accordance with requirements for electronic data as defined in the TWP (BSC 2004 [DIRS 171421]). No deviations from the TWP were necessary to complete this work. 


\section{INTENTIONALLY LEFT BLANK}




\section{USE OF SOFTWARE}

The water-level data were compiled and the potentiometric surface was constructed using a Geographic Information System (GIS), spreadsheet, and digitizing software. All of the software used in this analysis is controlled under LP-SI.11Q-BSC, Software Management, but is considered exempt for application in this analysis. Exempt is defined to mean that the software shall be controlled under the Software Configuration Management (SCM) system, but are not required to be qualified or documented under the procedure, except as noted in LP-SI.11Q-BSC, Section 2.1. Two justifications are provided for exempting the software based on LP-SI.11Q-BSC, Sections 2.1.2 and 2.1.6. First, the software is being used for visual display and graphical representation of data. Second, the calculations use standard functions of commercial off-the-shelf software programs. These calculations are not dependent on the software program used. Table 3-1 lists software used in this analysis that is considered qualified software in the centralized baseline.

Table 3-1. Software Used to Support Analysis Activity

\begin{tabular}{|c|c|c|l|l|}
\hline $\begin{array}{c}\text { Software } \\
\text { Name }\end{array}$ & Version & $\begin{array}{c}\text { Software Tracking } \\
\text { Number }\end{array}$ & $\begin{array}{c}\text { Computer Platform, } \\
\text { Operating System, Compiler }\end{array}$ & \multicolumn{1}{c|}{ Description } \\
\hline EarthVision & 5.1 & STN: 10174-5.1-00 & $\begin{array}{l}\text { Silicon Graphics (SGI) Octane } \\
\text { running IRIX 6.5 Operating } \\
\text { System } \\
\text { CPU ID\#: barcode 700800 } \\
\text { Location: Las Vegas, NV, YMP } \\
\text { Project Offices }\end{array}$ & $\begin{array}{l}\text { Coordinate transformation } \\
\text { Dynamic Graphics (2000 } \\
\text { [DIRS 167994]) }\end{array}$ \\
\hline
\end{tabular}

NOTE: $\mathrm{CPU}=$ central processing unit, STN = Software Tracking Number.

EarthVision Version 5.1 (STN: 10174-5.1-00) (Dynamic Graphics 2000 [DIRS 167994]) was used to transform coordinates from their original system to the UTM system used in this report. The application was within the range of use which is the set of real earth coordinates. The software was selected for use because it is known to project participants and is well respected in the scientific community. The coordinate transformation is a standard function of EarthVision and the application in this report is within the range of validation of the software. The location information is rounded to the nearest meter and any applications requiring more precise locations should not use the results in this analysis.

ARCINFO Version 7.2.1 (STN: 10033 7.2.1-01) (USGS 2000 [DIRS 148304]), published by Environmental Systems Research Institute, Inc., was used for plotting and visualization of analysis results. The range of validation for the ARCINFO application is the set of real numbers that define earth coordinate systems and elevations. Although this software is considered qualified in the centralized baseline, it is not qualified for use on SUN computers. The application of this software on a SUN computer is acceptable for this analysis because it is used in a way that exempts it per provisions in LP-SI.11Q-BSC, Sections 2.1.2 and 2.1.6. This commercial off-the-shelf software is available for both personal computers (with Microsoft Windows operating systems) and workstations (with UNIX operating systems, including those from SUN). Only standard functions of this commercial off-the-shelf were used to perform plotting and visualization. The potentiometric surface shown on Figure 6-1 was hand-contoured, precluding the need for contouring software. 
ARCINFO Version 8.0.2 (exempt per LP-SI.11Q-BSC, Software Management) was used to create a graphical display in Postscript format from files output from ARCINFO version 7.2.1. This software application performed no other function than to create the visual/graphical display in other file formats, and so was exempt according to LP-SI.11Q-BSC, Section 2.1.2.

CorelDraw 9, Version 9.337 (exempt per LP-SI.11Q-BSC) was used to create a graphical display in other graphics formats from the Postscript format generated by ARCINFO. This software application performed no other function than to create the visual/graphical display in other file formats, and so was exempt according to LP-SI.11Q-BSC, Section 2.1.2.

Microsoft Excel 97 SR2 (exempt per LP-SI.11Q-BSC) was used to compile water-level data, and to compute average water levels using the "AVERAGE" function of the software. Conversion of feet to meters was often done within Excel, by using the equation (meters $=$ feet $* 0.3048$ ). These simple conversions were spot checked with hand calculations. The original Excel spreadsheets (inputs) were obtained from the Technical Data Management System (TDMS) (see Table 4-1). The calculated average water levels (outputs) are listed in Appendix A, Table A-1, and are contained in DTN: GS010908312332.002.

AutoCAD Map 2000, release 4 (exempt per LP-SI.11Q-BSC) was used to digitize the hand-drawn potentiometric contours presented on Figure 6-1. The output digitized contours were used as input to ARCINFO for plotting. The final plots were visually compared with the hand-drawn contours.

Use of the software is documented in a scientific notebook (Tucci 2001 [DIRS 155410], pp. 6, 7, $8,18,58,70$ and 77). For the software above not included in Table 3-1, the computer platform and operating system were not recorded. Personal communication with Patrick Tucci in 2004 indicated that the computers used to do this work were replaced, but to the best of their knowledge, Intergraph TD-225 computers with Windows 2000 operating systems were used.

No model was used to support this analysis. The potentiometric-surface analysis does not require modeling to generate the final products. All that is needed is algebraic manipulation of depth to water data and surface elevation data. Simple contouring of the plotted data forms the bulk of the analysis. No calibration or fitting of data as would be expected for a modeling analysis is needed for this report.

Example calculations provided in the discussion of uncertainty were performed with a hand calculator and are fully documented in this report. 


\section{INPUTS}

\subsection{DIRECT INPUTS}

The data used to construct the potentiometric surface and to define water levels in selected boreholes for flow-model calibration were developed from measurements of water levels in boreholes throughout and adjacent to the SZ site-scale flow and transport model domain, through the year 2003. Included is (1) data from the original analysis (USGS 2001 [DIRS 154625]) with end dates from 1952 to 1999 , depending on availability of data at the time, (2) data from the alternative interpretation conducted in 2001 (USGS 2004 [DIRS 168473]) with end dates in 2000 for the Nye County wells available at the time, (3) data from Yucca Mountain site wells through 2001 to assess temporal variability of water levels, and (4) data from the Nye County wells through 2003 to assess the impact of the new well locations. The data from items (1) and (2) are the basis for the potentiometric-surface map presented in this report. New water-level data in items (3) and (4) are examined in the uncertainty section to assess the possible impacts to the potentiometric surface. The data used to construct the potentiometric-surface map, together with assessments of their accuracy and reliability, are presented in Tables A-1 through A-8 of Appendix A. In general, these water-level measurements represent the configuration of the potentiometric surface in the upper part of the SZ, and no additional control is available from springs or other surface-water occurrences. For some of the wells, the open intervals are long and the measured water levels may not be representative of the upper part of the aquifer. The uncertainty associated with these long-open-interval wells is discussed in Section 6.5.

These data are considered to be appropriate for their intended use in defining the upper boundary and determining lateral hydraulic gradients for the shallow portion of the SZ site-scale flow model. Some water levels not used in constructing the shallow potentiometric surface were obtained from deeper parts of the groundwater flow system and these data provide information on vertical hydraulic gradients that are important for calibration of the SZ site-scale flow model. Additional justification for the appropriateness of the data is documented in:

- Section 5.1 (Assumptions - Water-Level Data), which addresses assumptions concerning the use of the data

- Section 6.3 (Analysis-Water-Level Data), which discusses the analysis of the water-level data

- Section 6.5 (Analysis - Uncertainty in the Potentiometric-Surface Elevation Map), which describes and quantifies the various possible sources of uncertainty in the water levels and how those uncertainties may impact the potentiometric-surface map

- Section 7 (Conclusions), which summarizes the three products of this report; water-level data, vertical head differences, and the potentiometric-surface map.

Water-level data as defined above for the SZ site-scale flow and transport model area were evaluated; however, some data were considered invalid. Data that were identified as invalid by the United States Geological Survey (USGS) (2001 [DIRS 154625]) are not used in this revised analysis. Data that were considered for use in this analysis and documented in (USGS 2004 
[DIRS 168473]) were evaluated for validity and documented in a scientific notebook (Tucci 2001 [DIRS 155410]). Reasons for considering a water level invalid include short-term temporal fluctuations caused by seismic events, pumpage, equipment failure, or data collected prior to well development. Based on observations of water-level data at Yucca Mountain, water levels generally fluctuate less than $1 \mathrm{~m}$, and more often, less than $0.5 \mathrm{~m}$ (Graves et al. 1997 [DIRS 101046], Table 2). Savard (2001 [DIRS 165604]) presents an analysis of water-level data in the Yucca Mountain region through 1999 and showed that in only two cases is a temporal trend in water level observed. The temporal stability of the observed water levels is further confirmed in Table 6-3 where water levels, collected as recently as 2001, are compared with average values through 1995. Exceptions occur if a nearby well is pumped or if water levels are affected by seismic events. Most of the data that were considered invalid in this analysis consisted of data obtained before the well was developed, or were a result of known or suspected equipment failures. For example, water-level data are obtained from transducers set in discrete zones isolated by packers in Wells NC-EWDP-1S, NC-EWDP-3S, and NC-EWDP-9SX. Transducers are known to be subject to drift, failure, or electronic problems. Packers occasionally will fail to seal properly, causing an incomplete isolation of a zone, or can leak and fail as suggested by Savard (2001 [DIRS 165604], p. 55) for the lower interval of Borehole USW H-3. Transducer data were considered to be invalid if they indicated either slow drifting of water levels or sharp spikes. Such phenomena could be valid, but they would have to occur in more than one zone in a well and in more than one well to be considered valid. Such corresponding changes in other zones or wells rarely occurred, so that the anomalous data were considered invalid.

Water-level data collected in 1999 and 2001 in existing or new wells were also examined to determine if temporal trends in water levels impact the potentiometric surface. This assessment is presented in Section 6.3 as part of the discussion of uncertainty. The potentiometric-surface map was created without using the data from seven Nye County EWDP wells or well clusters south of Yucca Mountain but north of U.S. Highway 95. These seven wells, or well clusters (NC-EWDP-18P, NC-EWDP-27P, NC-EWDP-16P, NC-EWDP-10, NC-EWDP-28P, NC-EWDP-22, and NC-EWDP-23P) occupy a large region between the relatively high well density near the Yucca Mountain repository and the wells along U.S. Highway 95 (Figure 1-2). The water-level data from the seven Nye County wells collected in 2003 were used as an independent data set to assess the uncertainty associated with interpolation of water levels between widely spaced water-level measurement locations.

Groundwater flow may be affected by faults, which may act as barriers and/or conduits for flow. The locations of major faults in the SZ site-scale flow and transport model area were used to help guide the placement of potentiometric contours according to their assumed effect on groundwater flow (see Section 5.2). Fault locations and traces (Figure 6-1; DTN: GS991208314221.001 [DIRS 145263]) were used as direct input data during construction of the potentiometric-surface map. The geologic map in DTN: GS991208314221.001 was superseded by DTN: GS010908314221.001 [DIRS 162874], which among other things, modified the location of the U.S. Highway 95 fault. The new location of the U.S. Highway 95 fault (see RPC Pkg ID MOY-000619-04-03, Accession \# MOL.20020416.0184, Plate 1) will not lead to significant differences in the water-level contours presented on Figure 6-1. The contours are drawn to honor measured water-level data; the fault locations are used to guide the placement of contours. The data density from the Nye County wells is sufficient to constrain the location of contours around 
the U.S. Highway 95 fault, thus the new fault location will not significantly alter the contour locations. Per AP-SIII.9Q, Scientific Analyses, the superceded data must be demonstrated to be suitable for the intended use in this document. The input source for the original and superseding DTNs is the U.S. Geologic Survey in both cases. The USGS has a long history of creating geologic maps and is considered a reliable source for geologic maps. Individual scientists with the USGS are qualified geologists, thus both products were created by qualified personnel. Both data sets are suitable for this analysis and the superceding DTN will not alter contours because of constraints imposed by measured water levels. Therefore, the superceded DTN: GS991208314221.001 is suitable for use in the report.

One of the water-level data sets used in this analysis, DTN: GS950108312312.001 [DIRS 150993] is superseded by DTN: GS950808312312.008 [DIRS 171370]. The former DTN is justified for use in this report for two reasons: (1) the superceding DTN corrects only one data point, the water level from Borehole USW H-1 Tube 3 for December 20, 1994, and (2) the difference in water level on that date will not impact the results as presented in this report. From Appendix A, Table A-2, the number of data points for Borehole USW H-1 Tube 3 is 108. The difference in water level for Borehole USW H-1 Tube 3 on 12/20/1994 between the two DTNs is $0.23 \mathrm{~m}$. The mean water level presented in Table A-1 would change by an amount equal to $0.23 \mathrm{~m} / 108$ data points, or $0.002 \mathrm{~m}$. The mean water level is reported to the nearest tenth of a meter, so the use of superseded DTN: GS950108312312.001 [DIRS 150993] is justified for use in this document.

Another data set used in this analysis was superseded, but is suitable for use in this report. The DTN: MO0107COV01057.000 [DIRS 157194] has been superseded by DTN: MO0401COV03168.000 [DIRS 168534]. An Impact Review Action Notice (MOL.20040304.0206) documents there is no impact to the potentiometric-surface map. On a map such as Figure 6-1, 1 inch represents more than 6,000 meters of distance. A small difference in the lateral coordinate value would be a very small change on the figure. Consider a coordinate difference of $100 \mathrm{~m}$. The map portrays features at a scale of $6,000 \mathrm{~m}$ to $1 \mathrm{inch}$; a coordinate difference of $100 \mathrm{~m}$ produces an error of less than $0.02 \mathrm{inch}$. Moving any data point this small amount on the map will not significantly change the contours. These small errors in coordinates lead to acceptably small errors, thus justifying use of the superceded data throughout this analysis. Per AP.SIII-9Q, the data in the superceded DTN has been demonstrated to be suitable for the intended use in this document. As noted above, the changes to the DTN do not impact the generated maps. The superceded data is suitable for the intended use of portraying water-level measurement locations on Figures 6-1 and 6-2. The superceding data set DTN: MO0401COV03168.000 [DIRS 168534] is used to provide updated coordinate locations in Appendix A.

Specific direct input data sets, and associated DTNs, are listed in Table 4-1. The direct data are used to calculate the water-level elevation or provide data presented in one or more of the tables throughout the document. The qualification status of these input sources can be found in the Document Input Reference System (DIRS). 
Table 4-1. Direct Input Data Sources

\begin{tabular}{|c|c|}
\hline Data Description & Data Tracking Number \\
\hline Water-Level Measurements at UE-25 C \#2 and C \#3, 1989 & $\begin{array}{l}\text { GS000408312312.001 } \\
\text { [DIRS 148696] }\end{array}$ \\
\hline $\begin{array}{l}\text { Water-Level Altitude Data from the Periodic Network, January } 1999 \text { through } \\
\text { March } 1999\end{array}$ & $\begin{array}{l}\text { GS000608312312.003 } \\
\text { [DIRS 155278] }\end{array}$ \\
\hline Revised Water-Level Altitude Data from the Periodic Network, First Quarter 1995 & $\begin{array}{l}\text { GS000608312312.004 } \\
\text { [DIRS 150724] }\end{array}$ \\
\hline Water density as a function of temperature & Weast (1985) \\
\hline CRC Handbook of Chemistry and Physics, 66th Edition, Page F-10 & [DIRS 111561] \\
\hline Water-Level Altitude Data, 1993 & $\begin{array}{l}\text { GS000708312312.005 } \\
\text { [DIRS 150945] }\end{array}$ \\
\hline $\begin{array}{l}\text { Ground-Water Altitudes from Manual Depth-to-Water Measurements at Various } \\
\text { Boreholes November } 1998 \text { through December } 1999\end{array}$ & $\begin{array}{l}\text { GS000808312312.007 } \\
\text { [DIRS 155270] }\end{array}$ \\
\hline $\begin{array}{l}\text { Geohydrology of Rocks Penetrated by Test Well UE-25P\#1 (UE-25P\#1), Yucca } \\
\text { Mountain Area, Nye County, Nevada - Table S97274_001 }\end{array}$ & $\begin{array}{l}\text { GS920408312314.009 } \\
\text { Table S97274_001 } \\
\text { [DIRS 148168] } \\
\text { See Appendix B for the } \\
\text { justification of the use of } \\
\text { data }\end{array}$ \\
\hline $\begin{array}{l}\text { Geohydrologic Data and Test Results from Well J-13, Nevada Test Site, Nye } \\
\text { County, Nevada - Table 97276_013 }\end{array}$ & $\begin{array}{l}\text { GS930408312132.007 } \\
\text { Table S97276_013 } \\
\text { [DIRS 129625] } \\
\text { See Appendix B for the } \\
\text { justification of the use of } \\
\text { data }\end{array}$ \\
\hline Water Levels in the Yucca Mountain Area, Nevada, 1990-91 & $\begin{array}{l}\text { GS930408312312.015 } \\
\text { [DIRS 148665] }\end{array}$ \\
\hline $\begin{array}{l}\text { Water Levels in Periodically Measured Wells in the Yucca Mountain Area, Nevada, } \\
\text { 1981-1987 }\end{array}$ & $\begin{array}{l}\text { GS931008312312.025 } \\
\text { [DIRS 148668] }\end{array}$ \\
\hline Water-Level Altitude Data from the Periodic Network, Fourth Quarter, 1994 & $\begin{array}{l}\text { GS950108312312.001 } \\
\text { [DIRS 150993] } \\
\text { Justified for use in this } \\
\text { document } \\
\text { See Section } 4.1\end{array}$ \\
\hline Potentiometric-Surface Map, 1993, Yucca Mountain and Vicinity, Nevada & $\begin{array}{l}\text { GS950508312312.005 } \\
\text { [DIRS 105068] }\end{array}$ \\
\hline $\begin{array}{l}28 \text { Water-Level Measurements from the Periodic Network, Third Quarter, } 1995 \\
(7 / 1 / 95 \text { - 9/30/95) }\end{array}$ & $\begin{array}{l}\text { GS960208312312.003 } \\
{[\text { DIRS 148672] }}\end{array}$ \\
\hline Analysis of Water-Level Data in the Yucca Mountain Area, Nevada, 1985-1995 & $\begin{array}{l}\text { GS960908312312.010 } \\
\text { [DIRS 105063] }\end{array}$ \\
\hline Water Level Altitude Data Collected at GEXA Well 4 and USW G-4 & $\begin{array}{l}\text { GS970600012847.001 } \\
\text { [DIRS 148646] }\end{array}$ \\
\hline Water Levels in the Yucca Mountain Area, Nevada, 1996 & $\begin{array}{l}\text { GS980308312312.004 } \\
\text { [DIRS 155272] }\end{array}$ \\
\hline Water-Level Altitude Data, April - June 1999 & $\begin{array}{l}\text { GS990908312312.005 } \\
\text { [DIRS 155269] }\end{array}$ \\
\hline Water-Level Data for Yucca Mountain Region and Amargosa Desert & $\begin{array}{l}\text { GS991100002330.001 } \\
\text { [DIRS 122818] }\end{array}$ \\
\hline
\end{tabular}


Water-Level Data Analysis for the Saturated Zone Site-Scale Flow and Transport Model

Table 4-1. Direct Input Data Sources (Continued)

\begin{tabular}{|c|c|}
\hline Data Description & Data Tracking Number \\
\hline Geologic Map of the Yucca Mountain Region & $\begin{array}{l}\text { GS991208314221.001 } \\
\text { [DIRS 145263] } \\
\text { Justified for use in this } \\
\text { document } \\
\text { See Section } 4.1\end{array}$ \\
\hline $\begin{array}{l}\text { Water-Table-Altitude Data for Well USW G-4, Yucca Mountain Area, Nye County, } \\
\text { Nevada }\end{array}$ & $\begin{array}{l}\text { MO0008WTRALTG } 4.000 \\
\text { [DIRS 155456] }\end{array}$ \\
\hline $\begin{array}{l}\text { Locations and Elevations for Selected Wells in the Yucca Mountain Region and } \\
\text { Amargosa Desert from the USGS NWIS Database }\end{array}$ & $\begin{array}{l}\text { MO0011ELLOCAMD.000 } \\
\text { [DIRS 153274] }\end{array}$ \\
\hline Coverage: BORES3Q & $\begin{array}{l}\text { MO0103COV01031.000 } \\
\text { [DIRS 155271] }\end{array}$ \\
\hline Coverage: NCEWDPS. Submittal date: 07/18/2001 & $\begin{array}{l}\text { MO0107COV01057.000 } \\
\text { [DIRS 157194] } \\
\text { Justified for use in this } \\
\text { document } \\
\text { See Section } 4.1\end{array}$ \\
\hline Coverage: NCEWDPS: Submittal date: 01/27/2004 & $\begin{array}{l}\text { MO0401COV03168.000 } \\
\text { [DIRS 168534] }\end{array}$ \\
\hline Water-Level Data From Westbay Instrumented Borehole NC-EWDP-1S & $\begin{array}{l}\text { MO0111DQRWLNYE.002 } \\
\text { [DIRS 157172] }\end{array}$ \\
\hline Water-Level Data From Westbay Instrumented Borehole NC-EWDP-3S & $\begin{array}{l}\text { MO0111DQRWLNYE.003 } \\
\text { [DIRS 157173] }\end{array}$ \\
\hline Water-Level Data From Westbay Instrumented Borehole NC-EWDP-9SX & $\begin{array}{l}\text { MO0111DQRWLNYE.004 } \\
\text { [DIRS 157174] }\end{array}$ \\
\hline Well Completion Diagram For Borehole NC-EWDP-4PA & $\begin{array}{l}\text { MO0112DQRWLNYE.005 } \\
\text { [DIRS 157175] }\end{array}$ \\
\hline Well Completion Diagram For Borehole NC-EWDP-4PB & $\begin{array}{l}\text { MO0112DQRWLNYE.006 } \\
\text { [DIRS 157176] }\end{array}$ \\
\hline Well Completion Diagram For Borehole NC-EWDP-7S & $\begin{array}{l}\text { MO0112DQRWLNYE.007 } \\
\text { [DIRS 157177] }\end{array}$ \\
\hline Well Completion Diagram For Borehole NC-EWDP-5SB & $\begin{array}{l}\text { MO0112DQRWLNYE.008 } \\
\text { [DIRS 157178] }\end{array}$ \\
\hline Well Completion Diagram For Borehole NC-EWDP-15P & $\begin{array}{l}\text { MO0112DQRWLNYE.009 } \\
\text { [DIRS 157179] }\end{array}$ \\
\hline Well Completion Diagram For Borehole NC-EWDP-12PA & $\begin{array}{l}\text { MO0112DQRWLNYE.010 } \\
\text { [DIRS 157180] }\end{array}$ \\
\hline Well Completion Diagram For Borehole NC-EWDP-9SX & $\begin{array}{l}\text { MO0112DQRWLNYE.011 } \\
\text { [DIRS 157181] }\end{array}$ \\
\hline Well Completion Diagram For Borehole NC-EWDP-12PB & $\begin{array}{l}\text { MO0112DQRWLNYE.012 } \\
\text { [DIRS 157182] }\end{array}$ \\
\hline Well Completion Diagram For Borehole NC-EWDP-12PC & $\begin{array}{l}\text { MO0112DQRWLNYE.013 } \\
\text { [DIRS 157183] }\end{array}$ \\
\hline Well Completion Diagram For Borehole NC-EWDP-19P & $\begin{array}{l}\text { MO0112DQRWLNYE.014 } \\
\text { [DIRS 157184] }\end{array}$ \\
\hline Well Completion Diagram For Borehole NC-EWDP-3S & $\begin{array}{l}\text { MO0112DQRWLNYE.015 } \\
\text { [DIRS 157200] }\end{array}$ \\
\hline Well Completion Diagram For Borehole NC-EWDP-3D & $\begin{array}{l}\text { MO0112DQRWLNYE.016 } \\
\text { [DIRS 157185] }\end{array}$ \\
\hline
\end{tabular}


Table 4-1. Direct Input Data Sources (Continued)

\begin{tabular}{|l|l|}
\hline \multicolumn{1}{|c|}{ Data Description } & \multicolumn{1}{|c|}{ Data Tracking Number } \\
\hline Well Completion Diagram For Borehole NC-EWDP-1DX & $\begin{array}{l}\text { MO0112DQRWLNYE.017 } \\
\text { [DIRS 157186] }\end{array}$ \\
\hline Well Completion Diagram For Borehole NC-EWDP-19D & $\begin{array}{l}\text { MO0112DQRWLNYE.018 } \\
\text { [DIRS 157187] }\end{array}$ \\
\hline $\begin{array}{l}\text { Multi-Level Monitoring Port Depths In Nye County Boreholes NC-EWDP-1S, -3S, } \\
\text { and -9SX }\end{array}$ & $\begin{array}{l}\text { MO0112DQRWLNYE.019 } \\
\text { [DIRS 157188] }\end{array}$ \\
\hline Water-Level Depth Data For Nye County Boreholes NC-EWDP-2D and -2DB & $\begin{array}{l}\text { MO0112DQRWLNYE.020 } \\
\text { [DIRS 157199] }\end{array}$ \\
\hline Multilevel Piezometer Casing Log For Borehole NC-EWDP-9SX & $\begin{array}{l}\text { MO0112DQRWLNYE.021 } \\
\text { [DIRS 157189] }\end{array}$ \\
\hline Multilevel Piezometer Casing Log For Borehole NC-EWDP-3S & $\begin{array}{l}\text { MO0112DQRWLNYE.022 } \\
\text { [DIRS 157190] }\end{array}$ \\
\hline Multilevel Piezometer Casing Log For Borehole NC-EWDP-1S & $\begin{array}{l}\text { MO0112DQRWLNYE.023 } \\
\text { [DIRS 157191] }\end{array}$ \\
\hline $\begin{array}{l}\text { Ground-Water Altitudes from Manual Depth-to-Water Measurements at Various } \\
\text { Boreholes January through June 2001 }\end{array}$ & $\begin{array}{l}\text { GS010808312312.003 } \\
\text { [DIRS 168536] }\end{array}$ \\
\hline EWDP Phase I Manual Water-level Measurements & $\begin{array}{l}\text { MO0112DQRWLNYE.024 } \\
\text { [DIRS 157192] }\end{array}$ \\
\hline EWDP Phase II Manual Water-level Measurements & $\begin{array}{l}\text { MO0112DQRWLNYE.025 } \\
\text { [DIRS 157193] }\end{array}$ \\
\hline USW SD-7 Structure Logs (48.5' to 2675.1') & $\begin{array}{l}\text { TM0000SD7SUPER.001 } \\
\text { [DIRS 168540] }\end{array}$ \\
\hline USW SD-9 Structure Logs (53.6' to 2223.1') & $\begin{array}{l}\text { TM0000SD9SUPER.002 } \\
\text { [DIRS 168542] }\end{array}$ \\
\hline USW SD-12 Structure Logs (5.3' to 2166.3) & $\begin{array}{l}\text { TM000SD12SUPER.003 } \\
\text { [DIRS 168543] }\end{array}$ \\
\hline
\end{tabular}

The locations of boreholes, the water-level altitudes in these boreholes, and the potentiometric contours are shown on Figure 6-1. The borehole data are provided in Appendix A.

\subsection{CRITERIA}

The general requirements to be satisfied by the TSPA-LA are stated in 10 CFR 63.114 (10 CFR 63 [DIRS 156605]). Technical requirements to be satisfied by the TSPA-LA are identified in the Yucca Mountain Project Requirements Document (Canori and Leitner 2003 [DIRS 166275], Section 3). The pertinent project requirement for this report is number PRD-002/T-015, titled Requirements for Performance Assessment.

The acceptance criteria that will be used by the Nuclear Regulatory Commission (NRC) to determine whether the technical requirements that have been met are identified in the Yucca Mountain Review Plan, Final Report (YMRP; NRC 2003 [DIRS 163274]). The acceptance criteria for this report corresponding to project requirement PRD-002/T-015 are summarized in Table 4-2. In no case does the analysis presented in this report completely address the YMRP acceptance criteria. The analysis does provide results necessary for other model reports that further address the YMRP acceptance criteria. 
Table 4-2. Regulatory Requirements Addressed in This Report

\begin{tabular}{|c|c|c|}
\hline $\begin{array}{l}10 \text { CFR } 63 \\
\text { Citation }\end{array}$ & $\begin{array}{l}\text { YMRP Acceptance } \\
\text { Criteria }^{\mathrm{a}}\end{array}$ & YMRP Acceptance Criteria Definition ${ }^{a}$ \\
\hline $63.114(\mathrm{a}-\mathrm{c}),(\mathrm{g})$ & 2.2.1.3.8 AC1(2) & $\begin{array}{l}\text { The description of the aspects of hydrology, geology, geochemistry, } \\
\text { design features, physical phenomena, and couplings, that may affect } \\
\text { flow paths in the SZ, is adequate. Conditions and assumptions in the } \\
\text { abstraction of flow paths in the SZ are readily identified, and consistent } \\
\text { with the body of data presented in the description. }\end{array}$ \\
\hline $63.114(a-c),(g)$ & 2.2.1.3.8 AC1(4) & $\begin{array}{l}\text { Boundary and initial conditions used in the TSPA abstraction of flow } \\
\text { paths in the SZ are propagated throughout its abstraction approaches. } \\
\text { For example, abstractions are based on initial and boundary conditions } \\
\text { consistent with site-scale modeling and regional models of the Death } \\
\text { Valley groundwater flow system. }\end{array}$ \\
\hline $63.114(e)$ & 2.2.1.3.8 AC1(5) & $\begin{array}{l}\text { Sufficient data and technical bases to assess the degree to which FEPs } \\
\text { have been included in this abstraction are provided. }\end{array}$ \\
\hline $63.114(a),(g)$ & 2.2.1.3.8 AC1(6) & $\begin{array}{l}\text { Flow paths in the SZ are adequately delineated, considering natural site } \\
\text { conditions. }\end{array}$ \\
\hline $63.114(\mathrm{a}-\mathrm{c}),(\mathrm{e}-\mathrm{g})$ & 2.2.1.3.8 AC1(10) & $\begin{array}{l}\text { Guidance in NUREG-1297 and NUREG-1298, or other acceptable } \\
\text { approaches for peer review and data qualification is followed. }\end{array}$ \\
\hline $63.114(a),(b)$ & 2.2.1.3.8 AC2(1) & $\begin{array}{l}\text { Geological, hydrological, and geochemical values used in the LA to } \\
\text { evaluate flow paths in the SZ are adequately justified. Adequate } \\
\text { descriptions of how the data were used, interpreted, and appropriately } \\
\text { synthesized into the parameters are provided. }\end{array}$ \\
\hline $63.114(a),(b)$ & 2.2.1.3.8 AC2(2) & $\begin{array}{l}\text { Sufficient data have been collected on the natural system to establish } \\
\text { initial and boundary conditions for the abstraction of flow paths in the } \\
\text { SZ. }\end{array}$ \\
\hline 63.114(a),(b),(g) & 2.2.1.3.8 AC2(3) & $\begin{array}{l}\text { Data on the geology, hydrology, and geochemistry of the SZ used in the } \\
\text { TSPA abstraction are based on appropriate techniques. These } \\
\text { techniques may include laboratory experiments, site-specific field } \\
\text { measurements, natural analogue research, and process-level modeling } \\
\text { studies. As appropriate, sensitivity or uncertainty analyses, used to } \\
\text { support the DOE TSPA abstraction, are adequate to determine the } \\
\text { possible need for additional data. }\end{array}$ \\
\hline
\end{tabular}

a NRC 2003 [DIRS 163274].

\subsection{CODES, STANDARDS, AND REGULATIONS}

No codes, standards, or regulations other than those identified in the Project Requirements Document (Canori and Leitner 2003 [DIRS 166275], Table 2-3) and determined to be applicable (Table 4-2) as identified in the TWP (BSC 2004 [DIRS 171421], Section 3.0) were used in this analysis. The applicable requirements in 10 CFR Part 63 [DIRS 156605] are discussed in Section 4.2 above. 


\section{INTENTIONALLY LEFT BLANK}




\section{ASSUMPTIONS}

\subsection{WATER-LEVEL DATA}

In the analysis presented in this report, several assumptions are made. These assumptions follow established hydrologic practices for the determination of water-level altitudes to be used in the construction of a potentiometric-surface map and require no further confirmation.

- Assumption: Where measurement location (vertical) is unknown, the midpoint of the open interval or applicable packed-off interval is representative of the measurement location for SZ site-scale flow modeling purposes. Most of the water levels used in this analysis are composite data for a long open or screened interval. In open boreholes, the midpoint can be calculated as the mean of the altitude of the bottom of the borehole and the altitude of the maximum water-level altitude. In packed-off intervals (screened intervals), the midpoint is calculated as the mean of the altitude of the bottom of the packed-off interval and the altitude of the top of the packed-off interval. Because this method is used for all boreholes that contributed water-level altitude data for this analysis, it provides a means for standardizing SZ measurement locations (Appendix A, Table A-5). This assumption is used throughout the revision.

- Assumption: Water levels in Boreholes USW G-2, UE-25 WT \#6, and NC-EWDP-7S are perched. Water levels in Boreholes USW G-2 and UE-25 WT \#6, in the northern part of Yucca Mountain are assumed to represent perched conditions for the alternate concept of the large-hydraulic gradient area presented in this analysis. Czarnecki et al. (1997 [DIRS 141643], p. 27) present several lines of evidence to support this assumption. Water levels in Borehole NC-EWDP-7S in southern Crater Flat are also assumed to represent perched conditions. The water levels for Borhole NC-EWDP-7S are anomalously high (Figure 6-1; Appendix A, Table A-1), and no other hydrogeologic explanation other than perched conditions is plausible to explain such high water levels for this location. This assumption is used in Section 6.2.

- Assumption: Water levels in Borehole USW WT-24 at approximately $840 \mathrm{~m}$ above sea level represent the regional potentiometric level. During drilling, a water-bearing fracture was encountered at about $760 \mathrm{~m}$ below land surface, and water rose in the borehole to an altitude of about $840 \mathrm{~m}$ above sea level. Drilling continued for another $104 \mathrm{~m}$ below the water-bearing fracture without any significant change in the water level (Graves 2001 [DIRS 155942]). Because the potentiometric level persisted as the borehole was considerably deepened, and because the water level remained relatively stable after completion of the well, the $840 \mathrm{~m}$ level is assumed to represent the regional potentiometric level and not a perched level. This assumption is used in Section 6.2.

\subsection{POTENTIOMETRIC-SURFACE MAP}

- Assumption: Faults may act as barriers and/or conduits for groundwater flow. The concept used in the placement of potentiometric contours (Section 6.4, Figure 6-1) is that groundwater flow across a fault or fault zone is impeded by the fault, and groundwater flow parallel to a fault or fault zone is not impeded. The concept that faults 
may act as barriers to or conduits for groundwater flow is pointed out by Freeze and Cherry (1979 [DIRS 101173], p. 474). Within the SZ underlying Yucca Mountain, this assumption is further justified by the observations of Luckey et al. (1996 [DIRS 100465], pp. 25 and 56) that: (1) the Solitario Canyon fault along the west side of Yucca Mountain is an apparent barrier to flow from west to east across the fault and (2) where fault zones are intersected by boreholes, these zones act as conduits for flow and produce much of the water entering the boreholes (Luckey et al. 1996, [DIRS 100465], p. 37). 


\section{SCIENTIFIC ANALYSIS DISCUSSION}

The water-level data and the resulting potentiometric-surface map provide important technical input for the development of the SZ site-scale flow model. The input data for this analysis are considered direct input as outlined in AP-3.15Q, Managing Technical Product Input. The data from the Nye County EWDP wells (Phases III and IV) used to assess uncertainty in the potentiometric gradient south of Well ER-25 J-12 did not provide direct information in the development of the results or conclusions and are considered indirect input in this analysis. The Nye County data provide an independent data set for assessment of the impact of new data (and by analogy the impact of the uneven spatial distribution of data).

\subsection{OBJECTIVES OF THE ANALYSIS}

The potentiometric surface is regarded as the top of the SZ. It defines the upper elevation where radionuclides enter the $\mathrm{SZ}$ and begin moving downgradient to the accessible environment. The potentiometric-surface analysis provides important information for the SZ site scale flow model. First, the potentiometric-surface analysis provides an assessment of the hydraulic gradient in both horizontal and vertical directions. The gradient information aids in the development of the conceptual model of groundwater flow. The water-level data, in conjunction with the geologic and hydraulic property data, help to assess expected properties of the hydrostratigraphic features of the flow system including the expected role of faults. Second, the water-level calibration targets are defined in this analysis and characterized by the corresponding open interval (top and bottom elevation) and average hydraulic head value. These hydraulic head values in three-dimensional space are the water-level targets for calibration of the SZ site-scale flow model.

\subsection{FEATURES, EVENTS, AND PROCESSES FOR THIS ANALYSIS REPORT}

As stipulated in Technical Work Plan For: Natural System - Saturated Zone Analysis Model Report Integration (BSC 2004 [DIRS 171421]) this report addresses the SZ FEPs pertaining to water-level data analysis that are included for TSPA-LA (Table 6-1). SZ FEPs that were excluded for TSPA-LA are described in Features, Events, and Processes in SZ Flow and Transport (BSC 2004 [DIRS 170013]). Table 6-1 provides a list of FEPs that are relevant to this analysis in accordance with their assignment in the LA FEP list (DTN: MO0407SEPFEPLA.000 [DIRS 170760]). Specific reference to the various sections within this document where issues related to each FEP are addressed is provided in the table. The detailed discussion of these FEPs and their implementation in TSPA-LA are documented in the Features, Events, and Processes in SZ Flow and Transport (BSC 2004 [DIRS 170013]). 
Table 6-1. Features, Events, and Processes Included in TSPA-LA and Relevant to This Report

\begin{tabular}{|c|c|c|c|}
\hline FEP No. & FEP Name & $\begin{array}{l}\text { Sections Where Disposition } \\
\text { is Supported }\end{array}$ & $\begin{array}{l}\text { FEP Topic Addressed in Other SZ } \\
\text { Analysis or Model Reports }\end{array}$ \\
\hline 1.3.07.02.0A & $\begin{array}{l}\text { Water table rise } \\
\text { affects SZ }\end{array}$ & Section 6.4 & $\begin{array}{l}\text { Upstream Feeds }^{a}-\text { N/A } \\
\text { Corroborating }^{b}-\text { BSC } 2004 \text { [DIRS } \\
\text { 170036] }\end{array}$ \\
\hline 1.4.07.01.0A & $\begin{array}{l}\text { Water management } \\
\text { activities }\end{array}$ & Section 6.4 & $\begin{array}{l}\text { Upstream Feeds }{ }^{\mathrm{a}}-\mathrm{N} / \mathrm{A} \\
\text { Corroborating }^{\mathrm{b}}-\mathrm{BSC} 2004 \text { [DIRS } \\
\text { 170042] }\end{array}$ \\
\hline 1.4.07.02.0A & Wells & $\begin{array}{l}\text { Sections 1, 6.3, 6.4, 6.5, } \\
\text { Appendix A }\end{array}$ & $\begin{array}{l}\text { Upstream Feeds }{ }^{a}-N / A \\
\text { Corroborating }^{b}-\text { BSC } 2004 \text { [DIRS } \\
\text { 170042] }\end{array}$ \\
\hline
\end{tabular}

${ }^{a}$ Aspects of the SZ FEP screening position adopted in this report are a result of SZ analyses performed in a directly upstream SZ model or analyses. N/A indicates there are no upstream feeds.

${ }^{\mathrm{b}}$ Corroborating-SZ analysis or model report that indirectly supports the FEP topic.

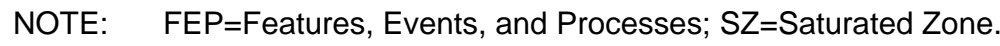

FEP 1.3.07.02.0A, Water table rise affects SZ is only partially addressed in this report. The water-level analysis provides the current state that is used by other analyses to assess the water table rise from future climate changes. FEP 1.4.07.01.0A, Water management activities, are not directly quantifiable, but are implicitly incorporated in the calibrated heads of the SZ flow model. As the potentiometric-surface analysis provides the heads for the calibration, this work indirectly supports this FEP. FEP 1.4.07.02.0A, Wells, is partially addressed in this report by presenting the locations of known water extraction wells (drinking water wells or agricultural wells) and the current water-level surface resulting from both natural conditions and the wells.

\subsubsection{Scientific Analysis Assumptions}

As part of the analysis of the data, a number of assumptions are necessary to complete the analysis. These assumptions are based primarily on accepted and established hydrologic practice. In each case, the justification for the assumption is provided.

- Assumption: Averaging water levels from the 1980s to 1990 s and up to 2000 provide water-level altitudes representative of conditions that existed in early 1990s. The SZ site-scale flow model (BSC 2004 [DIRS 170037]) uses groundwater fluxes from the Death Valley regional groundwater flow model (hereafter referred to as the regional model) (D'Agnese et al. 1997 [DIRS 100131], pp. 48 to 49) as calibration targets for lateral flux boundary conditions. The simulated fluxes in the regional model represent average, assumed steady-state conditions from the early 1990s, not conditions for a specific year. Therefore, the water levels used to construct the potentiometric-surface for the site-scale model must, to the extent allowed by data availability, represent conditions consistent with the regional model that used water-level data (altitudes) representing the early 1990s. Water levels in boreholes located at Yucca Mountain generally have not fluctuated by more than one meter during the time period from 1985 until 1995 (Graves et al. 1997 [DIRS 101046], Table 2). Savard (2001 [DIRS 165604]) extended the analysis through 1999 and showed that in nearly all cases, no temporal trend in water level is apparent. See Section 6.3 for a discussion of exceptions to this observation and the reasons why these exceptions are not important. Some of the 
boreholes used in this analysis had no or very few water-level measurements taken during the 1980s and 1990s (Appendix A, Tables A-2 and A-4). For boreholes in this category, all available water-level altitudes, with the exception of anomalous ones noted in Appendix A, were used to calculate the mean water-level altitude (Appendix A, Table A-1). This is particularly so for boreholes located in the Amargosa Valley and Amargosa Desert (Figure 6-1). Boreholes listed in Appendix A, Table A-2 as unreliable ("U") or assumed perched ("P") are shown on Figure 6-1, but were not used to construct the potentiometric surface. This assumption is used in Section 6.1 and Appendix A, Table A-1.

- Assumption: Mean water-level altitudes, even when influenced by groundwater withdrawal, represent water-level altitudes consistent with groundwater fluxes used in the SZ site-scale flow model. Some boreholes in the model area are pumped for commercial and domestic water supplies. Water-level altitudes in these and adjacent boreholes could be influenced by the effects of pumping. This condition is prevalent in the southern part of the SZ site-scale flow model area in the region of domestic and agricultural wells. The pumpage in the agricultural areas has been occurring over a long period of time (D'Agnese et al. 1997 [DIRS 100131], pp. 49 to 50), thus the potentiometric surface in that region has had a long period of time to respond. The present water levels are representative of the long-term pumping condition. The mean water-level altitude, calculated by averaging available data, provides a datum point that is representative of the potentiometric surface for the time period representing the early 1990s. The rationale is that average-annual pumping values were used in the regional model (D'Agnese et al. 1997 [DIRS 100131], pp. 48 to 49) and average water levels will be consistent with the simulated conditions in the regional model. This assumption is used in Sections 6.3 and 6.4 of this revision.

- Assumption: It is assumed that the water level from the uppermost open interval from each borehole at each site, except those boreholes listed in Assumption 2 in Section 5.1, represents the potentiometric surface of the uppermost part of the SZ (i.e., the water table). Most boreholes have only one water-level measurement interval; however, several boreholes have two or more isolated measurement intervals (Appendix A, Table A-5). In most boreholes, the uppermost interval is where the water was first encountered in the borehole (excluding perched-water zones, where identified). The significance of this assumption is that the resulting configuration of the potentiometric surface helps to determine the magnitude and direction of lateral groundwater flow in the upper SZ, which is important in the evaluation of potential radionuclide transport downgradient from the repository. As noted in Section 6.3.1, the vertical hydraulic head differences observed in the model area are largest when associated with the underlying carbonate aquifer or with the deeper portions of multiple completions (Wells USW H-1, USW H-3, USW H-6, and UE-25 p\#1). The upper portions of wells, even with multiple completions (see USW H-1) show small differences in water levels. These observations justify the use of the uppermost completion as representative of the water table because the observed water-level differences are small in the upper portion of the aquifer. This assumption is used in Section 6.4. 
- Assumption: Borehole locations used in this analysis, from DTN: MO0011ELLOCAMD.000 [DIRS 153274], are sufficiently accurate for the intended purpose. Borehole coordinates and altitudes from YMP (Yucca Mountain Site Characterization Project) boreholes in DTN: MO0011ELLOCAMD.000 [DIRS 153274] and in DTN: MO0107COV01057.000 [DIRS 157194], which only contains YMP boreholes, were compared. Differences in the northing and easting coordinates of as much as $82 \mathrm{~m}$ were observed by comparing the two DTNs. As explained in Section 4.1, these small differences in coordinate location produce acceptably small errors and either set of coordinates is justified for use in this analysis. The altitudes of the boreholes in the two data sets were identical. Borehole altitude is the most critical component of the borehole location used for calculating the water-level altitude.

\subsection{WATER-LEVEL DATA}

The water-level data for the potentiometric-surface analysis were compiled from project data sources and the USGS National Water Information System (NWIS) water-level data. The majority of the data used in this analysis was collected on or before 1996 as presented in USGS (2001 [DIRS 154625]). This data set was updated in USGS (2004 [DIRS 168473]) using new (collected through December 2000) information from the Nye County EWDP boreholes (Phases I and II), data from Borehole USW WT-24, and from geologic mapping within the model area (DTN: GS991208314221.001 [DIRS 145263]). Water-level information and analyses used as direct input were compiled from the data sources listed in Table 4-1 of this revision. The results were assembled in tabular format for use as input to the SZ site-scale flow model (see Appendix A). Other data were used to support this analysis and are considered indirect input. The indirect input data are identified in Table 6-2. The indirect data support the analysis, discussion, or visualization of results, but are not used to generate results applied directly in the calibration of the Site-Scale Saturated Zone Flow Model (BSC 2004 [DIRS 170037]). The indirect input includes water levels measured through 2001 from Yucca Mountain wells and the new Nye County water-level data (Phases III and IV) collected through 2003 that are used in the discussion of uncertainty. One source of uncertainty in the potentiometric-surface map is the interpolation of contours between unevenly distributed data points. To assess this uncertainty, an independent data set, not used to create the original map was needed. The data from Nye County Phases III and IV wells are withheld from the creation of the potentiometric surface to provide the independent data necessary to assess the uncertainty. 
Table 6-2. Indirect Input Data Sources

\begin{tabular}{|l|l|}
\hline \multicolumn{1}{|c|}{ Data Description } & \multicolumn{1}{|c|}{ Data Tracking Number } \\
\hline $\begin{array}{l}\text { As-Built Survey of Nye County Early Warning Drilling Program (EWDP) Phase III } \\
\text { Boreholes NC-EWDP-10S, NC-EWDP-18P, and NC-EWDP-22S - Partial Phase III } \\
\text { List }\end{array}$ & $\begin{array}{l}\text { MO0203GSC02034.000 } \\
\text { [DIRS 168375] }\end{array}$ \\
\hline $\begin{array}{l}\text { As-Built Survey of Nye County Early Warning Drilling Program (EWDP) Phase III } \\
\text { Boreholes, Second Set }\end{array}$ & $\begin{array}{l}\text { MO0206GSC02074.000 } \\
\text { [DIRS 168378] }\end{array}$ \\
\hline $\begin{array}{l}\text { As-Built Survey Of Nye County Early Warning Drilling Program Phase IV Boreholes } \\
\text { EWDP-16P, EWDP-27P \& EWDP-28P }\end{array}$ & $\begin{array}{l}\text { MO0307GSC03094.000 } \\
\text { [DIRS 170556] }\end{array}$ \\
\hline Digital Elevation Models Death Valley East Scale 1:250,000 & $\begin{array}{l}\text { GS0000400002332.001 } \\
\text { [DIRS 148924] }\end{array}$ \\
\hline $\begin{array}{l}\text { Manual Water-Level Data for EWDP Phase I Wells from 05/02-08/03, Phase II } \\
\text { Wells from 05/02-08/03, Phase III Wells from 11/02-08/03, and Phase IV Wells } \\
\text { from 01/03-08/03 }\end{array}$ & $\begin{array}{l}\text { MO0405NYE05819.215 } \\
\text { [DIRS 170539] }\end{array}$ \\
\hline
\end{tabular}

Water-level information used in this analysis was derived from a variety of sources. The large areal extent of the SZ site-scale flow and transport model, and the long history of water-level data collection in this area, have resulted in similar (or in some cases duplicate) water-level information being contained in multiple data sources. If more than one site ID was found in NWIS for the same borehole, the site ID with the most measurements was used for calculating the average water-level altitude for the time period of interest providing that the data were of equal quality to the data excluded. If location, land-surface altitude, or depth-to-water for a borehole were not available, the site was not used. NWIS data are stored as depth-to-water measurements, not as water-level altitude, and in English units as opposed to metric units. Conversion from depth-to-water to water-level altitude was accomplished by subtracting the depth-to-water measurement from the land-surface altitude of the measurement location (the borehole and surface altitude). The resulting water-level altitude was converted from feet to meters by using the equation (meters $=$ feet $* 0.3048$ ).

Borehole information was examined to see if water levels potentially represented perched-water conditions. Professional judgment was used to determine whether water-level altitudes represented perched-water conditions, based on the following criteria: proximity to cold-water springs, proximity to recharge areas, steep or anomalous potentiometric-surface slope, anomalous water-level altitudes, statistical water-level variability, water chemistry, pumping history, and hydrographs (O'Brien 1998 [DIRS 106918]). Potential perched-water levels identified during this analysis were flagged and identified as "suspected perched" or "assumed perched" (Appendix A, Tables A-2 and A-8). To prove perched-water occurrence unequivocally requires demonstrating partial saturation beneath a suspected perched-water body. Unfortunately, partial saturation could not be proved or disproved unequivocally with the available data for the boreholes in question, USW G-2, USW G-1, UE-29 a \#2, UE-25 a \#3, USW UZ-N91, UE-25 WT-18, UE-25 WT-6 (O’Brien 1998 [DIRS 106918]), and NC-EWDP-7S (DTN: MO0112DQRWLNYE.007 [DIRS 157177]). The boreholes in question were either drilled using a water-based circulating fluid (altering the ambient moisture distribution) or were only completed a few tens of meters into the first zone of saturation (not deep enough to reach the partial SZ). In this analysis, only water levels for Wells USW G-2, NC-EWDP-7S, and UE-25 WT \#6 are assumed to represent perched water areas because the water levels in those 
three wells depart from water levels in other nearby wells (see Section 5.1, Assumption 2). Water levels in the remaining wells are consistent with the alternative concept of the LHG presented in this report and with the water level in Borehole USW WT-24.

The data incorporated into the potentiometric-surface map for this revision are the same as in USGS (2004 [DIRS 168473]). Most data used in this report are from the period of 1985 to 1995 as reported in USGS (2001 [DIRS 154625]). Exceptions such as the Nye County well data from 1999 to 2000 are noted in Table A-4 of Appendix A. New data collected after 1995 were examined and determined to not impact the final product beyond the range of uncertainty presented in Section 6.5. Savard (2001 [DIRS 165604]) presents analysis of water levels in selected wells in 1999 and yearly average water levels in the same wells from 1985 to 1999 . As a general rule, water levels after 1995 do not fall outside the range of values from previous years. Most of the wells in the study area have long-term hydrograph records that show little water-level fluctuation over time. Several exceptions are noted. First, the most recent water levels in Wells USW H-3 upper interval and USW H-3 lower interval are now similar, but were historically different by almost $20 \mathrm{~m}$. It is suggested (Savard 2001 [DIRS 165604], p. 55) that the packer in Well USW H-3 failed and allowed the water levels in the two intervals to equilibrate. Therefore, the recent water levels in Well USW H-3 are not considered reliable. Another case where water levels have changed is in Well USW H-1, tubes 1 and 2. From 1985 to 1999 , water levels in Well USW H-1 tube 1 have risen almost 1 m, while in Well USW H-1 tube 2, the water level has fallen about $1 \mathrm{~m}$ over that same period (Savard 2000 [DIRS 165604], pp. 48 to 53). As presented in Table A-5, tubes 1 and 2 of Well USW H-1 are the deep completions and are not used in mapping the shallow potentiometric surface. Thus, the changes in water level in these two tubes will not impact the potentiometric-surface map. The rise in water level in tube 1 might increase the vertical water-level difference presented in Table 6-4 by about $1 \mathrm{~m}$, but this is only about a 2-percent change in the vertical head difference at location Well USW H-1 and is not considered significant because it will not alter any conclusions or analyses.

An assessment corroborating the stability of the water levels is presented in Table 6-3. Columns 3, 6, and 7 of Table 6-3 contain the same average, minimum, and maximum water levels as presented in Table A-1. Column 4 contains average water levels from the 6-month period from January 3, 2001 to June 26, 2001 (calculated from the data in DTN: GS010808312312.003 [DIRS 168536]). Column 5 is the average water-level data for the 12-month period of November 24, 1998, to December 22, 1999, (calculated as the mean of the data in DTN: GS000808312312.007 [DIRS 155270]). Compared with the average data through 1995, the more recent water levels fall within the previous minimum and maximum water levels with several exceptions. For two of the wells, USW G-2 and UE-25 WT \#16, the newer data fall outside the minimum or maximum value by about $0.2 \mathrm{~m}$. A single data point with this small difference will change the average water level by less than $0.02 \mathrm{~m}$. Another well with changes in water levels is USW H-1 tube 1 for 2001. According to Savard (2001 [DIRS 165604], Figure 39), the water levels in this well showed a trend of increasing average annual water levels from 1985 through 1999. The average water level in 2001 is slightly below any other year and may indicate a problem with the isolation of the interval or merely a long-term trend. Water levels in Well USW H-1 tube 2, also showed lower water levels in 2001 than in 1999. This decrease in water levels is consistent with the decreasing water trend noted by Savard (2001 [DIRS 165604], Figure 41). The recent water levels in Well USW H-3, upper and lower, may 
not be reliable as noted above due to possible packer failure. The impact of these trends in water levels will be discussed in Section 6.5. For nearly all the water levels, averaging the new data with the data used in this analysis would not have significantly altered the calculated average water level of the contours.

Table 6-3. Comparison of Water-Level Values through 1995 to Average Values in 1999 and 2001

\begin{tabular}{|c|c|c|c|c|c|c|c|}
\hline \multirow[b]{2}{*}{ USGS Site ID } & \multirow[b]{2}{*}{$\begin{array}{c}\text { Site } \\
\text { Name }\end{array}$} & \multirow{2}{*}{$\begin{array}{c}\text { Mean } \\
\text { Water-level } \\
\text { Altitude } \\
\text { through } \\
1995 \\
\text { (meters) } \\
\end{array}$} & \multirow{2}{*}{$\begin{array}{c}\text { Mean } \\
\text { Water-level } \\
\text { Altitude } \\
01 / 03 / 2001 \text { to } \\
06 / 26 / 2001 \\
\text { (meters) }\end{array}$} & \multirow{2}{*}{$\begin{array}{c}\text { Mean } \\
\text { Water-level } \\
\text { Altitude } \\
\text { 11/24/1998 } \\
\text { to } \\
\text { 12/22/1999 } \\
\text { (meters) }\end{array}$} & \multicolumn{2}{|c|}{$\begin{array}{c}\text { Water Levels } \\
\text { through } \\
1995 \text { (meters above } \\
\text { sea level) }\end{array}$} & \multirow[b]{2}{*}{ Source } \\
\hline & & & & & Minimum & Maximum & \\
\hline 365629116222602 & $\begin{array}{l}\text { UE-29 } \\
\text { a \#2 }\end{array}$ & 1187.7 & 1186.38 & 1186.58 & 1186.2 & 1191.3 & $\begin{array}{c}\text { APPENDIX A } \\
\text { GS000808312312.007 } \\
\text { GS010808312312.003 }\end{array}$ \\
\hline 365340116264601 & $\begin{array}{l}\text { UE-25 } \\
\text { WT \#6 }\end{array}$ & 1034.6 & 1034.9 & 1035.03 & 1033.3 & 1036.1 & $\begin{array}{c}\text { APPENDIX A } \\
\text { GS000808312312.007 } \\
\text { GS010808312312.003 }\end{array}$ \\
\hline 365322116273501 & $\begin{array}{l}\text { USW } \\
\text { G-2 }\end{array}$ & 1020.2 & 1019.69 & 1019.43 & 1019.6 & 1020.6 & $\begin{array}{c}\text { APPENDIX A } \\
\text { GS000808312312.007 } \\
\text { GS010808312312.003 }\end{array}$ \\
\hline 365239116253401 & \begin{tabular}{|l|} 
UE-25 \\
WT \#16
\end{tabular} & 738.3 & 738.83 & 738.36 & 737.8 & 738.6 & $\begin{array}{c}\text { APPENDIX A } \\
\text { GS000808312312.007 } \\
\text { GS010808312312.003 }\end{array}$ \\
\hline 365140116260301 & $\begin{array}{l}\text { UE-25 } \\
\text { WT \#4 }\end{array}$ & 730.8 & 730.92 & 730.83 & 730.3 & 731.2 & $\begin{array}{c}\text { APPENDIX A } \\
\text { GS000808312312.007 } \\
\text { GS010808312312.003 }\end{array}$ \\
\hline 365116116233801 & \begin{tabular}{|c|} 
UE-25 \\
WT \#15
\end{tabular} & 729.2 & 729.39 & 729.31 & 729 & 729.4 & $\begin{array}{c}\text { APPENDIX A } \\
\text { GS000808312312.007 } \\
\text { GS010808312312.003 }\end{array}$ \\
\hline 365032116243501 & \begin{tabular}{|l|} 
UE-25 \\
WT $\# 14$
\end{tabular} & 729.7 & 729.75 & 729.7 & 729.3 & 730 & $\begin{array}{c}\text { APPENDIX A } \\
\text { GS000808312312.007 } \\
\text { GS010808312312.003 }\end{array}$ \\
\hline 365023116271801 & $\begin{array}{l}\text { USW } \\
\text { WT-2 }\end{array}$ & 730.6 & 730.64 & 730.59 & 730.1 & 730.8 & $\begin{array}{c}\text { APPENDIX A } \\
\text { GS000808312312.007 } \\
\text { GS010808312312.003 } \\
\end{array}$ \\
\hline 364947116254401 & $\begin{array}{l}\text { UE-25 } \\
\text { C \#2 }\end{array}$ & 730.2 & 730.11 & 730.09 & 729.9 & 730.6 & $\begin{array}{c}\text { APPENDIX A } \\
\text { GS000808312312.007 } \\
\text { GS010808312312.003 }\end{array}$ \\
\hline 364945116235001 & \begin{tabular}{|l|} 
UE-25 \\
WT \#13
\end{tabular} & 729.1 & 729.3 & 729.23 & 728.5 & 729.4 & $\begin{array}{c}\text { APPENDIX A } \\
\text { GS000808312312.007 } \\
\text { GS010808312312.003 }\end{array}$ \\
\hline 364933116285701 & $\begin{array}{l}\text { USW } \\
\text { WT- } 7\end{array}$ & 775.8 & 776.03 & 775.95 & 775.5 & 776 & $\begin{array}{c}\text { APPENDIX A } \\
\text { GS000808312312.007 } \\
\text { GS010808312312.003 }\end{array}$ \\
\hline 364916116265601 & $\begin{array}{l}\text { USW } \\
\text { WT- } 1\end{array}$ & 730.4 & 730.42 & 730.32 & 730 & 730.5 & $\begin{array}{c}\text { APPENDIX A } \\
\text { GS000808312312.007 } \\
\text { GS010808312312.003 }\end{array}$ \\
\hline 364828116234001 & $\begin{array}{c}\text { UE-25 } \\
\mathrm{J}-13\end{array}$ & 728.4 & Not provided & 728.44 & 728.3 & 728.7 & $\begin{array}{c}\text { APPENDIX A } \\
\text { GS000808312312.007 }\end{array}$ \\
\hline 364825116290501 & $\begin{array}{c}\text { USW } \\
\text { WT-10 }\end{array}$ & 776 & 776.14 & 776.1 & 775.6 & 776.2 & $\begin{array}{c}\text { APPENDIX A } \\
\text { GS000808312312.007 } \\
\text { GS010808312312.003 }\end{array}$ \\
\hline 364822116262601 & \begin{tabular}{|l} 
UE-25 \\
WT \#17
\end{tabular} & 729.7 & 729.78 & 729.68 & 729.5 & 729.8 & $\begin{array}{c}\text { APPENDIX A } \\
\text { GS000808312312.007 } \\
\text { GS010808312312.003 }\end{array}$ \\
\hline
\end{tabular}


Water-Level Data Analysis for the Saturated Zone Site-Scale Flow and Transport Model

Table 6-3. Comparison of Water-level Values through 1995 to Average Values in 1999 and 2001 (Continued)

\begin{tabular}{|c|c|c|c|c|c|c|c|}
\hline \multirow[b]{2}{*}{ USGS Site ID } & \multirow{2}{*}{$\begin{array}{c}\text { Site } \\
\text { Name }\end{array}$} & \multirow{2}{*}{$\begin{array}{c}\text { Mean } \\
\text { Water-level } \\
\text { Altitude } \\
\text { through } \\
1995 \\
\text { (meters) } \\
\end{array}$} & \multirow{2}{*}{$\begin{array}{c}\text { Mean } \\
\text { Water-level } \\
\text { Altitude } \\
01 / 03 / 2001 \text { to } \\
06 / 26 / 2001 \\
\text { (meters) } \\
\end{array}$} & \multirow{2}{*}{$\begin{array}{c}\text { Mean } \\
\text { Water-level } \\
\text { Altitude } \\
\text { 11/24/1998 } \\
\text { to } \\
\text { 12/22/1999 } \\
\text { (meters) } \\
\end{array}$} & \multicolumn{2}{|c|}{$\begin{array}{c}\text { Water Levels } \\
\text { through } \\
1995 \text { (meters above } \\
\text { sea level) }\end{array}$} & \multirow[b]{2}{*}{ Source } \\
\hline & & & & & Minimum & Maximum & \\
\hline 364757116245801 & $\begin{array}{l}\text { UE-25 } \\
\text { WT \#3 }\end{array}$ & 729.6 & 729.78 & 729.69 & 729.4 & 729.9 & $\begin{array}{c}\text { APPENDIX A } \\
\text { GS000808312312.007 } \\
\text { GS010808312312.003 }\end{array}$ \\
\hline 364732116330701 & $\begin{array}{l}\text { USW } \\
\text { VH-1 }\end{array}$ & 779.4 & 779.48 & 779.49 & 779.3 & 779.6 & $\begin{array}{c}\text { APPENDIX A } \\
\text { GS000808312312.007 } \\
\text { GS010808312312.003 }\end{array}$ \\
\hline 364656116261601 & \begin{tabular}{|c|} 
UE-25 \\
WT $\# 12$
\end{tabular} & 729.5 & 729.54 & 729.45 & 729.1 & 729.6 & $\begin{array}{c}\text { APPENDIX A } \\
\text { GS000808312312.007 } \\
\text { GS010808312312.003 }\end{array}$ \\
\hline 364554116232400 & $\begin{array}{l}\mathrm{UE}-25 \\
\mathrm{~J}-12\end{array}$ & 727.9 & Not provided & 727.97 & 727.8 & 728.2 & $\begin{array}{c}\text { APPENDIX A } \\
\text { GS000808312312.007 }\end{array}$ \\
\hline 365157116271202 & $\begin{array}{c}\text { USW H-1 } \\
\text { tube } 1\end{array}$ & 785.5 & 784.51 & 786 & 785 & 786.1 & $\begin{array}{c}\text { APPENDIX A } \\
\text { GS000808312312.007 } \\
\text { GS010808312312.003 }\end{array}$ \\
\hline 365157116271203 & $\begin{array}{c}\text { USW H-1 } \\
\text { tube } 2\end{array}$ & 736 & 734.42 & 735.03 & 735.7 & 736.3 & $\begin{array}{c}\text { APPENDIX A } \\
\text { GS000808312312.007 } \\
\text { GS010808312312.003 }\end{array}$ \\
\hline 365157116271204 & $\left|\begin{array}{c}\text { USW H-1 } \\
\text { tube } 3\end{array}\right|$ & 730.6 & 730.62 & 730.69 & 730.4 & 730.8 & $\begin{array}{c}\text { APPENDIX A } \\
\text { GS000808312312.007 } \\
\text { GS010808312312.003 }\end{array}$ \\
\hline 365157116271205 & 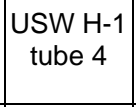 & 730.8 & 730.88 & 730.82 & 730.5 & 731 & $\begin{array}{c}\text { APPENDIX A } \\
\text { GS000808312312.007 } \\
\text { GS010808312312.003 }\end{array}$ \\
\hline 365122116275502 & $\begin{array}{c}\text { USW H-5 } \\
\text { upper }\end{array}$ & 775.5 & 775.52 & 775.48 & 775 & 775.7 & $\begin{array}{c}\text { APPENDIX A } \\
\text { GS000808312312.007 } \\
\text { GS010808312312.003 }\end{array}$ \\
\hline 365122116275503 & $\begin{array}{c}\text { USW H-5 } \\
\text { lower }\end{array}$ & 775.6 & 775.91 & 775.84 & 775 & 775.9 & $\begin{array}{c}\text { APPENDIX A } \\
\text { GS000808312312.007 } \\
\text { GS010808312312.003 }\end{array}$ \\
\hline 365049116285502 & $\left|\begin{array}{c}\text { USW H-6 } \\
\text { upper }\end{array}\right|$ & 776 & 776.15 & 776.1 & 775.8 & 776.2 & $\begin{array}{c}\text { APPENDIX A } \\
\text { GS000808312312.007 } \\
\text { GS010808312312.003 }\end{array}$ \\
\hline 365049116285505 & $\begin{array}{c}\text { USW H-6 } \\
\text { lower }\end{array}$ & 775.9 & 776.02 & 775.98 & 775.7 & 776.1 & $\begin{array}{c}\text { APPENDIX A } \\
\text { GS000808312312.007 } \\
\text { GS010808312312.003 }\end{array}$ \\
\hline 365032116265402 & $\begin{array}{c}\text { USW H-4 } \\
\text { upper }\end{array}$ & 730.4 & Not provided & 730.4 & 730.2 & 730.5 & $\begin{array}{c}\text { APPENDIX A } \\
\text { GS000808312312.007 }\end{array}$ \\
\hline 365032116265403 & $\begin{array}{c}\text { USW H-4 } \\
\text { lower }\end{array}$ & 730.5 & Not provided & 730.48 & 730.2 & 730.8 & $\begin{array}{c}\text { APPENDIX A } \\
\text { GS000808312312.007 }\end{array}$ \\
\hline 364942116280002 & $\left|\begin{array}{c}\text { USW H-3 } \\
\text { upper }\end{array}\right|$ & 731.5 & 732.06 & 731.86 & 731.1 & 731.9 & $\begin{array}{c}\text { APPENDIX A } \\
\text { GS000808312312.007 } \\
\text { GS010808312312.003 }\end{array}$ \\
\hline 364942116280003 & $\begin{array}{l}\text { USW H-3 } \\
\text { lower }\end{array}$ & 755.9 & 732.24 & 732.09 & 747.4 & 760.3 & $\begin{array}{c}\text { APPENDIX A } \\
\text { GS000808312312.007 } \\
\text { GS010808312312.003 } \\
\end{array}$ \\
\hline 364938116252102 & $\begin{array}{l}\text { UE-25 } \\
\text { p \#1 } \\
\text { (Lwr } \\
\text { Intrvl) }\end{array}$ & 752.4 & 753.13 & 752.93 & 751.9 & 752.7 & $\begin{array}{c}\text { APPENDIX A } \\
\text { GS000808312312.007 } \\
\text { GS010808312312.003 }\end{array}$ \\
\hline
\end{tabular}


Table 6-3. Comparison of Water-level Values through 1995 to Average Values in 1999 and 2001 (Continued)

\begin{tabular}{|c|c|c|c|c|c|c|c|}
\hline \multirow[b]{2}{*}{ USGS Site ID } & \multirow[b]{2}{*}{$\begin{array}{c}\text { Site } \\
\text { Name }\end{array}$} & \multirow{2}{*}{$\begin{array}{c}\text { Mean } \\
\text { Water-level } \\
\text { Altitude } \\
\text { through } \\
1995 \\
\text { (meters) } \\
\end{array}$} & \multirow{2}{*}{$\begin{array}{c}\text { Mean } \\
\text { Water-level } \\
\text { Altitude } \\
01 / 03 / 2001 \text { to } \\
06 / 26 / 2001 \\
\text { (meters) } \\
\end{array}$} & \multirow{2}{*}{$\begin{array}{c}\text { Mean } \\
\text { Water-level } \\
\text { Altitude } \\
11 / 24 / 1998 \\
\text { to } \\
12 / 22 / 1999 \\
\text { (meters) } \\
\end{array}$} & \multicolumn{2}{|c|}{$\begin{array}{c}\text { Water Levels } \\
\text { through } \\
1995 \text { (meters above } \\
\text { sea level) }\end{array}$} & \multirow[b]{2}{*}{ Source } \\
\hline & & & & & Minimum & Maximum & \\
\hline 365301116271301 & $\begin{array}{c}\text { USW } \\
\text { WT-24 }\end{array}$ & 840.1 & 840.61 & 840.29 & 839.7 & 840.7 & $\begin{array}{c}\text { APPENDIX A } \\
\text { GS000808312312.007 } \\
\text { GS010808312312.003 }\end{array}$ \\
\hline 364706116170601 & $\begin{array}{l}\text { UE-25 } \\
\mathrm{J}-11\end{array}$ & 732.2 & Not provided & 732.26 & 732.1 & 732.4 & $\begin{array}{c}\text { APPENDIX A } \\
\text { GS000808312312.007 }\end{array}$ \\
\hline 365624116222901 & $\begin{array}{l}\text { USW } \\
\text { UZ-N91 }\end{array}$ & 1186.7 & 1187.03 & 1187.26 & 1185.6 & 1191.3 & $\begin{array}{c}\text { APPENDIX A } \\
\text { GS000808312312.007 } \\
\text { GS010808312312.003 }\end{array}$ \\
\hline
\end{tabular}

Revision 01 of this report used the data from EWDP Phases I and II. Some additional Nye county wells (Phases III and IV) were drilled and unqualified water levels are currently available (DTN: MO0405NYE05819.215 [DIRS 170539]). The new wells (NC-EWDP Wells 10S, 10P, 16P, 18P, 19IM1, 19IM2, 22PA, 22PB, 22S, 23P, 27P, and 28P) occupy eight locations located in the vicinity of Fortymile Wash north of U.S. U.S. Highway 95 (as shown on Figure 1-2). The data from these wells are addressed in Section 6.5.

\subsubsection{Qualification of Unqualified Data}

Two data sets were qualified for intended use within this document. The data are measured water levels in Wells UE-25 p\#1 and UE-25 J-13. Both data sets are qualified using procedure AP-SIII.2Q and documented in Appendices B and C.

\subsubsection{Vertical Head Differences}

Within the SZ site-scale flow and transport model area, 17 boreholes monitor, or have historically monitored, water levels in more than one vertical interval (Table 6-4). The water-level data in Table 6-4 are compiled for the purpose of assessing vertical hydraulic head. The time frame of data in Table 6-4 is limited to ensure a direct comparison of the water levels in the different intervals. The data in Appendix A represent long-term average water levels used to construct the potentiometric-surface map. For some locations, the water-level data in Table 6-4 do not match the data presented in Appendix A. In most cases, the vertical measurements were made during a short period of time when access to multiple zones within the borehole was available (the water supply Well UE-25 J-13 is an example). The data in Appendix A and Table 6-4 are justified for their respective purposes and do not need to be the same.

The pattern of water-level differences identified for single years in Table 6-4 is repeated over longer periods of time as discussed by Savard (2001 [DIRS 165604], pp. 50 to 67) for Boreholes USW H-1, USW H-3, USW H-4, USW H-5, and USW H-6. The data for the Nye County EWDP wells are the same in Table 6-4 and Appendix A. Water-level data from these boreholes allow for the calculation of the difference in potentiometric heads at each monitored interval. 
Both upward (head increases with depth) and downward (head decreases with depth) vertical gradients have been observed. Fewer downward gradients (5) are observed than upward gradients (12). Upward vertical head differences range from $0.1 \mathrm{~m}$ to almost $55 \mathrm{~m}$, and downward vertical head differences range from 0.5 to $38 \mathrm{~m}$. The monitored intervals were selected to either monitor water levels between different geologic units or between different permeable intervals within the same geologic unit.

Table 6-4. Vertical Head Differences

\begin{tabular}{|c|c|c|c|c|c|}
\hline Well & 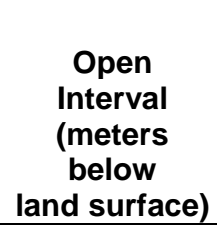 & $\begin{array}{c}\text { Potentiometric } \\
\text { Level } \\
\text { (meters above } \\
\text { sea level) }\end{array}$ & $\begin{array}{c}\text { Head } \\
\text { Difference } \\
\text { deepest- } \\
\text { shallowest } \\
\text { intervals } \\
\text { (meters) }\end{array}$ & Source Data & Remarks \\
\hline USW $\mathrm{H}-1$ tube 4 & $573-673$ & 730.94 & 54.7 & GS930408312312.015 & $\begin{array}{l}1991 \text { mean level } \\
\text { (Luckey et al. } 1996 \\
\text { [DIRS 100465], } \\
\text { Table 3) }\end{array}$ \\
\hline USW H-1 tube 3 & $716-765$ & 730.75 & & GS930408312312.015 & $\begin{array}{l}1991 \text { mean level } \\
\text { (Luckey et al. } 1996 \\
\text { [DIRS 100465], } \\
\text { Table 3) }\end{array}$ \\
\hline USW H-1 tube 2 & $1097--1123$ & 736.06 & & GS930408312312.015 & $\begin{array}{l}1991 \text { mean level } \\
\text { (Luckey et al. } 1996 \\
\text { [DIRS 100465], } \\
\text { Table 3) }\end{array}$ \\
\hline USW $\mathrm{H}-1$ tube 1 & 1783-1814 & 785.58 & & GS930408312312.015 & $\begin{array}{l}1991 \text { mean level } \\
\text { (Luckey et al. } 1996 \\
\text { [DIRS 100465], } \\
\text { Table 3) }\end{array}$ \\
\hline USW H-3 upper & $762-1114$ & 731.19 & 28.9 & GS980308312312.004 & $\begin{array}{l}1996 \text { mean level } \\
\text { (Graves } 1998 \\
\text { [DIRS 155411], p. 59) }\end{array}$ \\
\hline USW H-3 lower & 1114-1219 & 760.07 & & GS980308312312.004 & $\begin{array}{l}1996 \text { mean level } \\
\text { (Graves } 1998 \\
\text { [DIRS 155411], p. 59 }\end{array}$ \\
\hline USW H-4 upper & 525-1188 & 730.49 & 0.1 & GS930408312312.015 & $\begin{array}{l}1991 \text { mean level } \\
\text { (Luckey et al. } 1996 \\
\text { [DIRS 100465], } \\
\text { Table 3) }\end{array}$ \\
\hline USW H-4 lower & 1188-1219 & 730.56 & & GS930408312312.015 & $\begin{array}{l}1991 \text { mean level, } \\
\text { (Luckey et al. } 1996 \\
\text { [DIRS 100465], } \\
\text { Table 3) }\end{array}$ \\
\hline USW H-5 upper & 708-1091 & 775.43 & 0.2 & GS930408312312.015 & $\begin{array}{l}1991 \text { mean level, } \\
\text { (Luckey et al. } 1996 \\
\text { [DIRS 100465], } \\
\text { Table 3) }\end{array}$ \\
\hline USW H-5 lower & $1091-1219$ & 775.65 & & GS930408312312.015 & $\begin{array}{l}1991 \text { mean level, } \\
\text { (Luckey et al. } 1996 \\
\text { [DIRS 100465], } \\
\text { Table 3) }\end{array}$ \\
\hline
\end{tabular}


Table 6-4. Vertical Head Differences (Continued)

\begin{tabular}{|c|c|c|c|c|c|}
\hline Well & 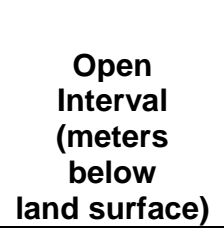 & $\begin{array}{c}\text { Potentiometric } \\
\text { Level } \\
\text { (meters above } \\
\text { sea level) }\end{array}$ & $\begin{array}{c}\text { Head } \\
\text { Difference } \\
\text { deepest- } \\
\text { shallowest } \\
\text { intervals } \\
\text { (meters) }\end{array}$ & Source Data & Remarks \\
\hline USW H-6 upper & $533-752$ & 775.99 & 2.2 & GS930408312312.015 & $\begin{array}{l}1991 \text { mean level, } \\
\text { (Luckey et al. } 1996 \\
\text { [DIRS 100465], } \\
\text { Table 3) }\end{array}$ \\
\hline USW H-6 lower & $752-1220$ & 775.91 & & GS930408312312.015 & $\begin{array}{l}1991 \text { mean level, } \\
\text { (Luckey et al. } 1996 \\
\text { [DIRS 100465], } \\
\text { Table 3) }\end{array}$ \\
\hline USW H-6 & $1193-1220$ & 778.18 & & GS931008312312.025 & $\begin{array}{l}\text { 1/84-5/84 mean level, } \\
\text { (Luckey et al. } 1996 \\
\text { [DIRS 100465], } \\
\text { Table 3) }\end{array}$ \\
\hline UE-25 b \#1 upper & 488-1199 & 730.71 & -1.0 & GS930408312312.015 & $\begin{array}{l}1991 \text { mean level, } \\
\text { (Luckey et al. } 1996 \\
\text { [DIRS 100465], } \\
\text { Table 3) }\end{array}$ \\
\hline UE-25 b \#1 lower & $1199-1220$ & 729.69 & & GS930408312312.015 & $\begin{array}{l}1991 \text { mean level, } \\
\text { (Luckey et al. } 1996 \\
\text { [DIRS 100465], } \\
\text { Table 3) }\end{array}$ \\
\hline $\begin{array}{l}\text { UE-25 } p \text { \#1 } \\
\text { (volcanic) }\end{array}$ & $384-500$ & 729.90 & 21.4 & $\begin{array}{l}\text { GS920408312314.009 } \\
\text { Table S97274_001 }\end{array}$ & $\begin{array}{l}\text { Uppermost interval } \\
\text { value (Craig and } \\
\text { Robinson 1984 } \\
\text { [DIRS 101040], } \\
\text { Table 2) }\end{array}$ \\
\hline $\begin{array}{l}\text { UE-25 } p \text { \#1 } \\
\text { (carbonate) }\end{array}$ & 1297-1805 & 751.26 & & $\begin{array}{l}\text { GS920408312314.009 } \\
\text { Table S97274_001 }\end{array}$ & \begin{tabular}{|l} 
Average of last 12 \\
entries (Craig and \\
Robinson 1984 \\
[DIRS 101040], \\
Table 2) \\
\end{tabular} \\
\hline UE-25 c \#3 & $692-753$ & 730.22 & 0.4 & GS930408312312.015 & $\begin{array}{l}1990 \text { mean level } \\
\text { (Luckey et al. } 1996 \\
\text { [DIRS 100465], } \\
\text { Table 3) }\end{array}$ \\
\hline UE-25 c \#3 & $753-914$ & 730.64 & & GS930408312312.015 & $\begin{array}{l}1990 \text { mean level } \\
\text { (Luckey et al. } 1996 \\
\text { [DIRS 100465], } \\
\text { Table 3) }\end{array}$ \\
\hline & $615-747$ & 730.3 & -0.5 & MO0008WTRALTG4.000 & $\begin{array}{l}\text { Luckey et al. } 1996 \\
\text { [DIRS 100465], Table } 3\end{array}$ \\
\hline USW G-4 & $747-915$ & 729.8 & & MO0008WTRALTG4.000 & $\begin{array}{l}\text { Luckey et al. } 1996 \\
\text { [DIRS 100465], Table } 3\end{array}$ \\
\hline UE-25 J-13 upper & $282-451$ & 728.8 & -0.8 & $\begin{array}{l}\text { GS930408312132.007 } \\
\text { Table S97276_013 }\end{array}$ & $\begin{array}{l}\text { Thordarson 1983 } \\
\text { [DIRS 101057], } \\
\text { Table 10 }\end{array}$ \\
\hline UE-25 J-13 & $471-502$ & 728.9 & & $\begin{array}{l}\text { GS930408312132.007 } \\
\text { Table S97276_013 }\end{array}$ & $\begin{array}{l}\text { Thordarson } 1983 \\
\text { [DIRS 101057], } \\
\text { Table 10 }\end{array}$ \\
\hline
\end{tabular}


Table 6-4. Vertical Head Differences (Continued)

\begin{tabular}{|c|c|c|c|c|c|}
\hline Well & 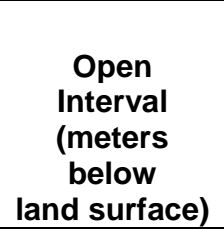 & \begin{tabular}{|c} 
Potentiometric \\
Level \\
(meters above \\
sea level)
\end{tabular} & $\begin{array}{c}\text { Head } \\
\text { Difference } \\
\text { deepest- } \\
\text { shallowest } \\
\text { intervals } \\
\text { (meters) }\end{array}$ & Source Data & Remarks \\
\hline UE-25 J-13 & $585-646$ & 728.9 & & $\begin{array}{l}\text { GS930408312132.007 } \\
\text { Table S97276_013 }\end{array}$ & \begin{tabular}{|l} 
Thordarson 1983 \\
[DIRS 101057], \\
Table 10
\end{tabular} \\
\hline UE-25 J-13 & 820-1063 & 728.0 & & $\begin{array}{l}\text { GS930408312132.007 } \\
\text { Table S97276_013 }\end{array}$ & \begin{tabular}{|l} 
Thordarson 1983 \\
[DIRS 101057], \\
Table 10
\end{tabular} \\
\hline $\begin{array}{l}\text { NC-EWDP-1DX } \\
\text { (shallow) }\end{array}$ & WT-419 & 786.8 & -38.0 & $\begin{array}{l}\text { MO0112DQRWLNYE.017 } \\
\text { MO0112DQRWLNYE.024 }\end{array}$ & $5 / 99-2 / 00$ \\
\hline $\begin{array}{l}\text { NC-EWDP-1DX } \\
\text { (deep) }\end{array}$ & $658-683$ & 748.8 & & $\begin{array}{l}\text { MO0112DQRWLNYE.024 } \\
\text { MO0112DQRWLNYE.017 }\end{array}$ & 8/99-2/00 \\
\hline $\begin{array}{l}\text { NC-EWDP-2D } \\
\text { (volcanic) }\end{array}$ & WT-493 & 706.1 & 7.2 & $\begin{array}{l}\text { MO0112DQRWLNYE.020 } \\
\text { MO0112DQRWLNYE.024 }\end{array}$ & $1 / 99$ \\
\hline $\begin{array}{l}\text { NC-EWDP-2DB } \\
\text { (carbonate) }\end{array}$ & $820-937$ & 713.3 & & $\begin{array}{l}\text { MO0112DQRWLNYE.020 } \\
\text { MO0112DQRWLNYE.025 }\end{array}$ & $11 / 15 / 00-11 / 22 / 00$ \\
\hline $\begin{array}{l}\text { NC-EWDP-3S } \\
\text { probe } 2\end{array}$ & $103-129$ & 719.8 & -1.5 & $\begin{array}{l}\text { MO0112DQRWLNYE.015 } \\
\text { MO0112DQRWLNYE.019 } \\
\text { MO0111DQRWLNYE.003 } \\
\text { MO0112DQRWLNYE.022 }\end{array}$ & 5/06/99-12/06/00 \\
\hline $\begin{array}{l}\text { NC-EWDP-3S } \\
\text { probe } 3\end{array}$ & $145-168$ & 719.4 & & $\begin{array}{l}\text { MO0112DQRWLNYE.015 } \\
\text { MO0112DQRWLNYE.019 } \\
\text { MO0111DQRWLNYE.003 } \\
\text { MO0112DQRWLNYE.022 }\end{array}$ & 5/06/99-12/06/00 \\
\hline NC-EWDP-3D & WT-762 & 718.3 & & $\begin{array}{l}\text { MO0112DQRWLNYE.024 } \\
\text { MO0112DQRWLNYE.016 }\end{array}$ & 3/99-8/99 \\
\hline NC-EWDP-4PA & $124-148$ & 717.9 & 5.7 & $\begin{array}{l}\text { MO0112DQRWLNYE.025 } \\
\text { MO0112DQRWLNYE.005 }\end{array}$ & $1 / 13 / 00-10 / 26 / 00$ \\
\hline NC-EWDP-4PB & $225-256$ & 723.6 & & $\begin{array}{l}\text { MO0112DQRWLNYE.025 } \\
\text { MO0112DQRWLNYE.006 }\end{array}$ & $1 / 21 / 00-10 / 26 / 00$ \\
\hline $\begin{array}{l}\text { NC-EWDP-9SX } \\
\text { probe } 1\end{array}$ & $27-37$ & 766.7 & 0.1 & $\begin{array}{l}\text { MO0112DQRWLNYE.011 } \\
\text { MO0112DQRWLNYE.019 } \\
\text { MO0111DQRWLNYE.004 } \\
\text { MO0112DQRWLNYE.021 }\end{array}$ & 5/13/99-12/06/00 \\
\hline $\begin{array}{l}\text { NC-EWDP-9SX } \\
\text { probe } 2\end{array}$ & $43-49$ & 767.3 & & $\begin{array}{l}\text { MO0112DQRWLNYE.011 } \\
\text { MO0112DQRWLNYE.019 } \\
\text { MO0111DQRWLNYE.004 } \\
\text { MO0112DQRWLNYE.021 }\end{array}$ & 5/13/99-12/06/00 \\
\hline $\begin{array}{l}\text { NC-EWDP-9SX } \\
\text { probe } 4\end{array}$ & 101-104 & 766.8 & & $\begin{array}{l}\text { MO0112DQRWLNYE.011 } \\
\text { MO0112DQRWLNYE.019 } \\
\text { MO0111DQRWLNYE.004 } \\
\text { MO0112DQRWLNYE.021 }\end{array}$ & 5/13/99-12/06/00 \\
\hline NC-EWDP-12PA & 99-117 & 722.9 & 2.2 & $\begin{array}{l}\text { MO0112DQRWLNYE.025 } \\
\text { MO0112DQRWLNYE.010 }\end{array}$ & $4 / 18 / 00-11 / 15 / 00$ \\
\hline NC-EWDP-12PB & 99-117 & 723.0 & & $\begin{array}{l}\text { MO0112DQRWLNYE.025 } \\
\text { MO0112DQRWLNYE.012 }\end{array}$ & $4 / 18 / 00-11 / 15 / 00$ \\
\hline NC-EWDP-12PC & $52-70$ & 720.7 & & $\begin{array}{l}\text { MO0112DQRWLNYE.025 } \\
\text { MO0112DQRWLNYE.013 }\end{array}$ & $4 / 27 / 00-11 / 15 / 00$ \\
\hline
\end{tabular}


Table 6-4. Vertical Head Differences (Continued)

\begin{tabular}{|c|c|c|c|c|c|}
\hline Well & $\begin{array}{c}\begin{array}{c}\text { Open } \\
\text { Interval } \\
\text { (meters } \\
\text { below }\end{array} \\
\text { land surface) }\end{array}$ & $\begin{array}{c}\text { Potentiometric } \\
\text { Level } \\
\text { (meters above } \\
\text { sea level) }\end{array}$ & $\begin{array}{c}\text { Head } \\
\text { Difference } \\
\text { deepest- } \\
\text { shallowest } \\
\text { intervals } \\
\text { (meters) }\end{array}$ & Source Data & Remarks \\
\hline NC-EWDP-19P & $109-140$ & 707.5 & 5.3 & $\begin{array}{l}\text { MO0112DQRWLNYE.025 } \\
\text { MO0112DQRWLNYE.014 }\end{array}$ & $3 / 13 / 00-6 / 17 / 00$ \\
\hline NC-EWDP-19D & $106-433$ & 712.8 & & $\begin{array}{l}\text { MO0112DQRWLNYE.025 } \\
\text { MO0112DQRWLNYE.018 }\end{array}$ & $6 / 14 / 00-6 / 22 / 00$ \\
\hline
\end{tabular}

Source: Output DTN: GS010908312332.003.

NOTE: Negative value indicates downward gradient.

Only two sites (UE-25 p\#1 and NC-EWDP-2D/2DB see Table 6-4) provide information on vertical gradients between volcanic rocks and the underlying Paleozoic carbonate rocks. At Well UE-25 p\#1, water levels currently are monitored only in the carbonate aquifer; however, water-level data were obtained from within the volcanic rocks as the borehole was drilled and tested (DTN: GS920408312314.009 [DIRS 148168]). At this site, water levels in the Paleozoic carbonate rocks are about $20 \mathrm{~m}$ higher than those in the overlying volcanic rocks. Borehole NC-EWDP-2DB penetrated Paleozoic carbonate rocks toward the bottom of the borehole (Spengler 2001 [DIRS 155823]). Water levels measured within that deep part of the borehole are about $8 \mathrm{~m}$ higher than levels measured in volcanic rocks penetrated by Borehole NC-EWDP-2D.

Water levels monitored within the lower part of the volcanic-rock sequence at Yucca Mountain also are significantly higher than levels monitored in the upper part of the volcanics. Boreholes USW H-1 (tube 1) and USW H-3 (lower interval) both monitor water levels in the lower part of the volcanic-rock sequence and upward gradients are observed at these boreholes with head differences of $54.7 \mathrm{~m}$ and $28.9 \mathrm{~m}$, respectively (Table 6-4). The gradient at Well USW H-3 is not precisely known, because the water levels in the lower interval had been continuously rising before the packer that separates the upper and lower intervals failed in 1996 (Graves 1998 [DIRS 155411], pp. 58 to 61; DTN: GS980308312312.004 [DIRS 155272]).

An upward gradient is also observed between the alluvial deposits monitored in Borehole NC-EWDP-19P and underlying volcanic rocks monitored in Borehole NC-EWDP-19D (Table 6-4). The vertical head difference at this site is $5.3 \mathrm{~m}$; however, levels reported for NC-EWDP-19D represent a composite water level for both alluvium and volcanics, so that the true head difference between those units is not completely known.

Downward gradients are observed within the SZ site-scale flow and transport model area (Table 6-4). The largest downward gradient is observed between the deep and shallow monitored intervals at Borehole NC-EWDP-1DX (head difference of $38 \mathrm{~m}$ ). The depth to water at this site is shallow $(17 \mathrm{~m})$ and within Tertiary spring deposits. Other downward gradients are much smaller in magnitude. Vertical downward gradients were also observed at Well NC-EWDP-7S (see Section 6.4). 
Five wells or well sites with more than two monitoring intervals (Table 6-4; Well USW H-1, Well USW H-6, Well UE-25 J-13, site NC-EWDP-3D/NC-EWDP-3S, and Well NC-EWDP-9SX) have head differences between the uppermost and lowermost monitored intervals and the intermediate intervals in which the gradients are both upwards and downwards. For example at Well USW H-1, the head difference between the uppermost (tube 4) and next lower interval (tube 3 ) is $-0.2 \mathrm{~m}$, indicating a small downward gradient; however, the head difference between tube 3 and the next lower interval (tube 2) is $5.3 \mathrm{~m}$, indicating an upward gradient.

Several reasons may be advanced to explain the small gradient reversals at some locations. First, small scale heterogeneity may be present that produces these small differences. The impact of these heterogeneities would be expected to be most important at small scales, but would be averaged out when viewed along the entire flow path of more than $18 \mathrm{~km}$. Second, the small reversals may be due simply to measurement errors. Section 6.5 presents an analysis of numerous sources of error that could impact the measured values. Small differences, especially on the order of a few tenths of a meter, require careful analysis to ensure that such differences are not the result of errors.

The confidence in the vertical hydraulic head differences is greatest for the locations with the largest head differences because these observed differences are much larger than any potential errors. As noted in Section 6.5, uncertainty in the water-level measurement may come from a variety of sources. As a result, there is less confidence in small head differences because the uncertainty in the water level may be larger than the head difference.

\subsubsection{Key Technical Issues (KTI) Related to Potentiometric Surface and Vertical Gradients}

Williams (2003 [DIRS 170977], Appendix C) provides a response to the additional information needed (AIN) request from the Nuclear Regulatory Commission (NRC) for KTI agreement

Unsaturated and Saturated Flow Under Isothermal Conditions (USFIC) 5.08. The wording of these agreements is:

\section{USFIC 5.08}

- Taking into account the Nye County Information, provide the updated potentiometric data and map for the regional aquifer, and an analysis of vertical hydraulic gradients within the site-scale model. DOE will provide an updated potentiometric map and supporting data for the uppermost aquifer in an update to the Water-Level Data Analysis for the Saturated Zone Site-Scale Flow and Transport Model report expected to be available in October 2001, subject to receipt of data from the Nye County program. Analysis of vertical hydraulic gradient will be addressed in the site-scale model and will be provided in the Calibration of the Site-Scale Saturated Zone Flow Model report expected to be available during FY 2002. 


\section{USFIC 5.08 AIN-1}

- Incorporate data for Well SD-6, which was drilled several years ago and provided key information about hydraulic heads close to the Solitario Canyon fault, into the analysis of water levels near Yucca Mountain and provide the analysis for NRC review. The same data given in tables in the water-level report for other Wells should be provided for SD-6.

- Provide a hydrogeologic interpretation for the high heads observed in Wells UZ-14 and H-5.

- Provide an updated hydrogeologic interpretation for groundwater elevations in Wells G-2 and WT\#6 (i.e., wells that define the LHG) based on newly available data from Well WT-24.

- Provide the basis for assuming that the water level in Well NC-EWDP-7S represents perched water.

\section{Data for Well SD-6}

The water-level information for Borehole USW SD-6 is provided in two DTNs: GS000808312312.007 [DIRS 155270] and GS001208312312.009 [DIRS 171433]. The three water-level elevations in those two DTNs range from 731.10 to $731.70 \mathrm{~m}$. A water level of $731.2 \mathrm{~m}$ was used as part of model validation in the calibration of the SZ site-scale flow model (BSC 2001 [DIRS 155974], pp. 48 to 51). This is a more direct use of Borehole USW SD-6 water-level data than is the incorporation of this information into the potentiometric-surface map (Figure 6-1). An argument presented by Williams (2003 [DIRS 170977]) is that the SD-6 data would not have changed the potentiometric-surface map. This can be seen by observing the location of USW SD-6 on Figure 1-2 and noting that the contours on Figure 6-1 would not have changed with the addition of the new wells. The exclusion of Borehole USE SD-6 is justified on the basis of no impact.

\section{High heads in Wells USW UZ-14 and USW H-5}

The high potentiometric level in Borehole USW H-5 has been attributed to the presence of a splay of the Solitario Canyon fault penetrated by the borehole (Ervin et al. 1994 [DIRS 100633], pp. 9 to 10). This splay is believed to be an extension of the hydrologic barrier to west-to-east groundwater flow from Crater Flat (related to the Solitario Canyon fault). The high heads in Well USW H-5 (about $775 \mathrm{~m}$ ) are related to heads in Crater Flat (ranging from 775 to $780 \mathrm{~m}$ ), and this borehole defines part of the moderate hydraulic gradient along the western edge of Yucca Mountain. Borehole USW UZ-14 is in a transition zone between the large and moderate hydraulic gradient areas, and the high potentiometric level (about $779 \mathrm{~m}$ ) is related to either of these areas. Rousseau et al. (1999 [DIRS 102097], p. 172) hypothesized that perched water in Borehole USW UZ-14 could be caused by a nearby projected growth fault that impedes percolation of water from the surface. This fault may also impede groundwater flow in the SZ. The high heads in Borehole USW UZ-14 also could be caused by the low-permeability rocks in the upper part of the SZ at that borehole. 
Update the hydrogeologic interpretation for water levels in Wells USW G-2 and UE-25 WT\#6 based on newly available data from Well USW WT-24

The discussion of the interpretation of water levels in Wells USW G-2, UE-25 WT\#6, and USW WT-24 is presented in Sections 5.1, 6.4 and 7.1.2.

\section{Basis for assuming that the water level in Well NC-EWDP-7S represents perched water}

The discussion of the perched water level in Well NC-EWDP-7S is presented at the end of Section 6.4.

\subsection{POTENTIOMETRIC-SURFACE MAP}

For SZ site-scale flow model construction purposes, the 2001 potentiometric-surface map (Figure 6-1) was created from the water-level data listed in USGS (2004 [DIRS 168473], Table I-1). Table I-1 of USGS (2004 [DIRS 168473]) uses the same input data as defined in Table A-1 of this report except that DTN: MO0107COV01057.000 [DIRS 157194] is used instead of DTN: MO0401COV01368.000 [DIRS 168534]. The differences in data from USGS (2004 [DIRS 168473]) and Table A-1 of this document are in the coordinate values due to revisions to a data set for the Nye County wells (see Section 4.1 for details). As explained in Section 4.1, the differences in coordinate values are acceptable because they do not impact the display of data in Figure 6-1. The 2001 potentiometric contours were hand-drawn by the USGS (2004 [DIRS 168473], Figure 6-1) rather than computer-generated as in revision 00 of this report (USGS 2001 [DIRS 154625], Figure 1-2). This hand contouring allowed the Principal Investigator (PI) to locate the contours based on professional hydrologic judgment and experience. Hand contouring and machine contouring are both accepted methods to generate water-level contour maps. Machine contours are easily reproducible but may suffer from odd extrapolations to areas of limited data and may generate physically unrealistic contours if allowed to honor all data values, regardless of associated errors in the values. Acceptable machine contouring requires careful oversight by a hydrologist to prevent unrealistic results. The careful oversight of the machine contours in the initial 2000 potentiometric contour map (USGS 2001 [DIRS 154625], Figure 1-2) is evident because the map does not exhibit odd behavior. To achieve this level of oversight, a hydrologist must apply judgment to constrain the contouring. Thus, professional judgment is a necessary component of water-level contouring regardless of how the final contours are generated (machine or hand). Hand contouring is considered equivalent to machine contouring for this analysis because both involve the use of professional judgment.

In accordance with the discussion in Section 6.2.1 (Assumption 3), the water-level altitude from the upper interval of each borehole was assumed to represent the potentiometric surface. Under this assumption, only water-level altitudes representing the uppermost aquifer system, typically the volcanic or alluvial system, were used to construct the map on Figure 6-1. Fault traces shown on Figure 6-1 represent the mapped surface expression of faults.

The distribution of water-level data and the complex geology in the SZ site-scale flow model area allow for various interpretations of the configuration of the potentiometric surface (Luckey et al. 1996 [DIRS 100465], pp. 21 to 26). Several potentiometric-surface maps 
(discussed in Section 6.5) have been developed that encompass Yucca Mountain and the surrounding vicinity, including the SZ site-scale flow and transport model area and the regional model area. Examination of other potentiometric-surface maps that fully or partially cover the SZ site-scale flow and transport model area reveals no major differences in the shape of potentiometric contour lines. This is not unexpected because similar, and in some cases the same, water-level data were used to create the potentiometric contour lines. By adhering to the rules that govern the construction of potentiometric contours, only a limited number of configurations of the water-level data are possible. The differences observed between existing potentiometric-surface maps and the one presented in this report can be attributed to map scale, potentiometric-contour intervals, changing concepts of potential perched water and the regional potentiometric surface in the LHG area, and more and newer water-level data. The potentiometric-surface map created for this report is an accurate interpretation based on the available water-level data through the year 1996 (the year 1998 for some wells in Amargosa Valley and the year 2000 for the EWDP wells), the geologic map of the Yucca Mountain region, the assumptions in Section 5, and the regional potentiometric surface.

The potentiometric analysis provides supplemental justification for several FEPs. The potentiometric surface provides a snapshot of current water-level conditions. Analyses to address water table rise caused by increased infiltration in the future will use the present analysis as the starting point. At the present time there are no significant water management structures such as dams, reservoirs, or canals in the vicinity of Yucca Mountain. The report has identified all the water-level observation points, including wells drilled for human consumption and agricultural use (Figure 1-2). The potentiometric-surface analysis identifies expected directions of groundwater flow from the repository to the accessible environment. The well locations presented in this report can be compared later to flow and transport model results to identify wells likely to be in the contaminant plume. There are no springs identified in the site-scale model area. The potentiometric surface is always below the land surface. Thus, there are no locations of natural discharge to the land surface in the site-scale model area.

This analysis differs from that reported in Revision 00 of this report (USGS 2001 [DIRS 154625], Figure 1-2) in several ways. The most significant difference is in the portrayal of the LHG area north of Yucca Mountain. The concept that water levels in Boreholes USW G-2 and UE-25 WT \#6 are considered to represent perched conditions (Assumption 2 in Section 5.1), is used to create the 2001 potentiometric surface in this revision. By not using the data from those two boreholes, the LHG is reduced from about 0.11 (Tucci and Burkhardt 1995 [DIRS 101060], p. 9) to between 0.06 to 0.07 , and the potentiometric contours are more widely spaced. Another significant difference is that potentiometric contours are no longer offset where they cross faults. Such offsets, which were shown in the 2000 map presented in Revision 00 of this report (USGS 2001 [DIRS 154625], Figure 1-2), would not be expected where the contours are perpendicular or nearly perpendicular to the fault trace. Direct evidence of offset, which would be provided by wells that straddle the fault, does not exist at Yucca Mountain. Faults were used, however, to help in the placement of contours that are oriented parallel or approximately parallel to faults. The concept used to represent the impact of faults on potentiometric contours is that groundwater flow across a fault (or fault zone) is impeded by the fault and that groundwater flow parallel to a fault is not impeded by the fault. The basis for this representation is discussed in Section 5.2. 


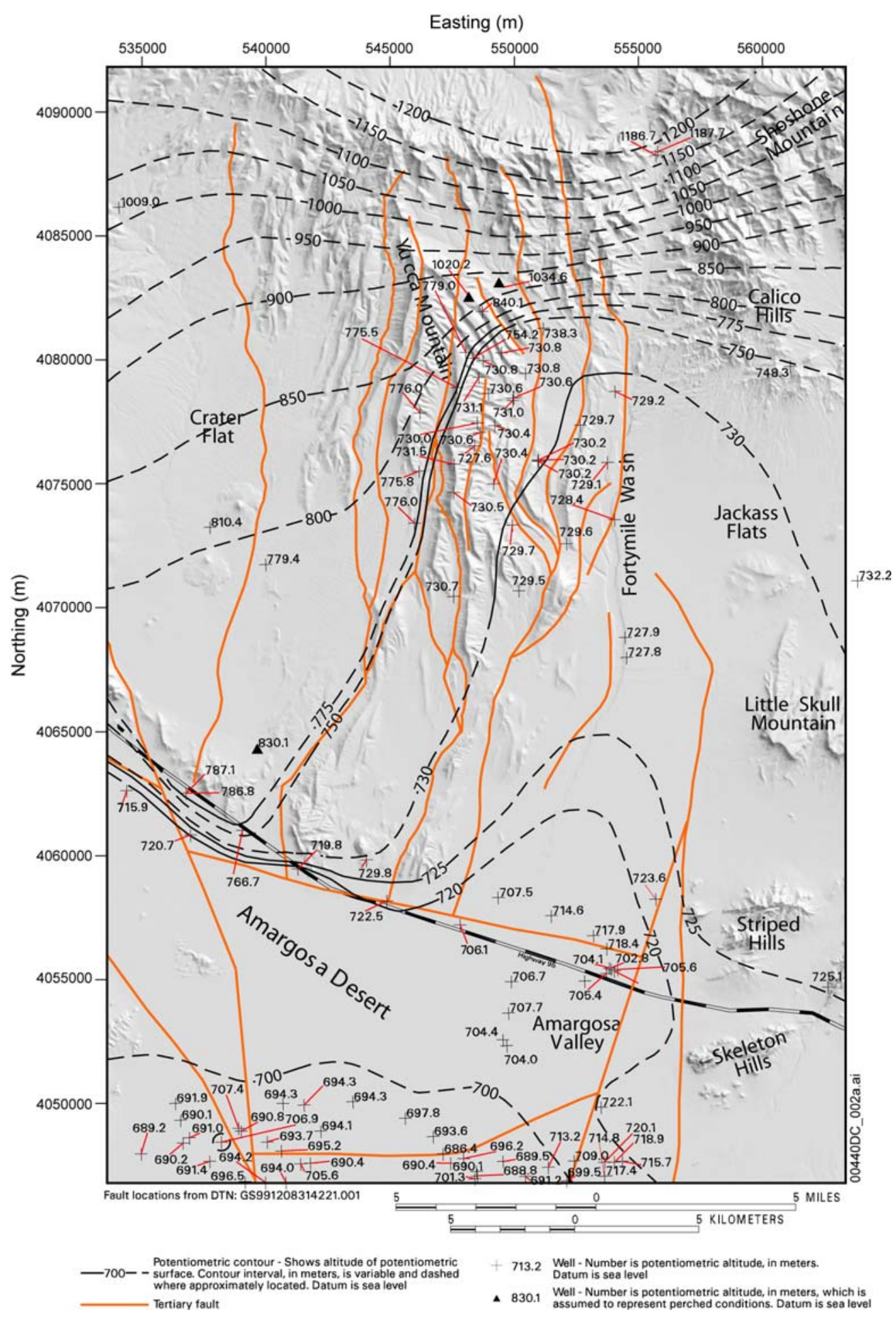

DTN: GS010608312332.001.

Source: USGS 2004 [DIRS 168473].

Figure 6-1. 2001 Potentiometric-Surface Map, Assuming Perched Conditions North of Yucca Mountain, in the SZ Site-Scale Flow and Transport Model Area 
The contour interval used in this analysis is somewhat different from Revision 00 of this report (USGS 2001 [DIRS 154625], Figure 1-2), which used a uniform contour interval of $25 \mathrm{~m}$ (USGS 2001 [DIRS 154625], Figure 1-2). The contour interval used on Figure 6-1 is variable, in which the interval is $50 \mathrm{~m}$ for contours greater than $800 \mathrm{~m}$, and $25 \mathrm{~m}$ for contours less than $800 \mathrm{~m}$. Two additional contours, 730 and 720 , are also included. The inclusion of those contours helps to visualize the effect of the fault along U.S. Highway 95 (Figure 6-1), south of Yucca Mountain, on the groundwater flow system.

Potentiometric data indicate a complex three-dimensional flow system. Luckey et al. (1996 [DIRS 100465], pp. 21 to 26, and 56) discuss different gradients and interpretations of the SZ site-scale flow system. Groundwater flow in the welded volcanic rocks occurs primarily in fractures and secondarily in the matrix of the rock (Ervin et al. 1994 [DIRS 100633], p. 8; Luckey et al. 1996 [DIRS 100465], pp. 17 to 21). Therefore, this flow system may result from the presence of faults and associated fracture zones occurring in the welded volcanic hydrogeologic units, rather than a system in which groundwater flow is through a porous medium. Depending upon where the potentiometric surface is located within the hydrostratigraphic sequence, it may be either confined or unconfined. Confined aquifers may exist where a relatively less permeable hydrogeologic unit, such as a clay bed or argillic volcanic unit, overlies a permeable hydrogeologic unit. An unconfined aquifer has no overlying relatively less permeable hydrogeologic unit, or if an overlying less permeable unit is present, the water level lies below the base of the less permeable unit.

Many of the boreholes used in this analysis only partially penetrate a single hydrogeologic unit. In boreholes that do penetrate more than one hydrogeologic unit, no attempt was made to distinguish water-level measurements associated with specific hydrogeologic units or fracture zones. The water-level altitudes in some boreholes represent composite heads from multiple hydrogeologic units and fractures zones. Generally, water levels in the uppermost SZ appear to represent a laterally continuous, well-connected aquifer system (Tucci and Burkhardt 1995 [DIRS 101060], p. 7). The impact on the potentiometric surface from boreholes that are open at different depth intervals and to different hydrogeologic units is discussed in relation to uncertainty in Section 6-5.

Some of the major faults in the region are thought to affect water levels (Ervin et al. 1994 [DIRS 100633], p. 9; Tucci and Burkhardt 1995 [DIRS 101060]; Luckey et al. 1996 [DIRS 100465], pp. 21 to 26; D’Agnese et al. 1997 [DIRS 100131]). As a result, several of these faults were selected to help interpret the water-level data used in the analysis (Figure 6-1). The selection was based on fault displacement of geologic units and extent of the fault, both laterally and vertically. The location of some of the major faults may explain the water-level altitudes in some of the boreholes and the resulting potentiometric-surface map. For example, an area termed the "moderate hydraulic gradient" is associated with the area adjacent to the Solitario Canyon fault, which is located along the west side of Yucca Mountain (approximately parallel to the 750-m and 775-m contours shown on Figure 6-1). Water-level altitudes to the west of the Solitario Canyon fault are more than $40 \mathrm{~m}$ higher than those to the east (Ervin et al. 1994 [DIRS 100633], pp. 7 to 9; Tucci and Burkhardt 1995 [DIRS 101060], pp. 7 to 9). A moderate-to-LHG area southwest of Yucca Mountain also appears to be related to a fault that is approximately parallel to U.S. Highway 95 (Figure 6-1). 
The potentiometric surface is characterized by four major regions that can be inferred from the potentiometric contours depicted on Figure 6-1:

- A small-gradient (0.0001 to 0.0004; Tucci and Burkhardt 1995 [DIRS 101060], p. 9) area below Yucca Mountain, to the east, and to the southeast of Yucca Mountain where water levels range from about 728 to $732 \mathrm{~m}$. Gradients in the Amargosa Desert, south of Yucca Mountain are also small (0.001 to 0.0004; water levels ranging from about $690 \mathrm{~m}$ to about $720 \mathrm{~m}$ )

- A moderate-gradient (0.02 to 0.04; Tucci and Burkhardt 1995 [DIRS 101060], p. 9) area to the west of Yucca Mountain, where water levels range from about $740 \mathrm{~m}$ to $800 \mathrm{~m}$

- A moderate- to large-gradient (0.01 to 0.05$)$ area southwest of Yucca Mountain (along U.S. Highway 95 near southern Crater Flat), where water levels range from $720 \mathrm{~m}$ to $775 \mathrm{~m}$

- A large-gradient (0.06 to 0.07) area north of Yucca Mountain, where water levels range from about $738 \mathrm{~m}$ to $1188 \mathrm{~m}$. This gradient assumes that water levels in Boreholes USW G-2 and UE-25 WT \#6 represent perched conditions. The hydraulic gradient in the LHG area north of Yucca Mountain previously had been reported as about 0.11 (Tucci and Burkhardt 1995 [DIRS 101060], p. 9).

The potentiometric surface presented in this analysis and in previously published reports generally implies a hydraulically, well-connected flow system within the uppermost SZ (Tucci and Burkhardt 1995 [DIRS 101060]) as discussed above.

A number of explanations have been proposed to explain the presence of the apparent LHG at the north end of Yucca Mountain. Explanations proposed for the LHG include:

- Faults that contain nontransmissive fault gouge (Czarnecki and Waddell 1984 [DIRS 101042], p. 19)

- Faults that juxtapose transmissive tuff against nontransmissive tuff (Czarnecki and Waddell 1984 [DIRS 101042], p. 19)

- The presence of a less fractured lithologic unit (Czarnecki and Waddell 1984 [DIRS 101042], p. 19)

- A change in the direction of the regional stress field and a resultant change in the intensity, interconnectedness, and orientation of open fractures on either side of the area with the LHG (Czarnecki and Waddell 1984 [DIRS 101042], p. 19)

- The apparent large gradient actually represents a disconnected, perched- or semi-perched- water body, so that the high water-level altitudes are caused by local hydraulic conditions and are not part of the regional SZ flow system (Ervin et al. 1994 [DIRS 100633]). 
Fridrich et al. (1994 [DIRS 100575]) suggest two hydrogeologic explanations for the LHG: (1) a highly permeable buried fault that drains water from the volcanic rock units into a deeper regional carbonate aquifer or (2) a buried fault that forms a "spill-way" in the volcanic rocks. Their second explanation, in effect, juxtaposes transmissive tuff against nontransmissive tuff, and is therefore the same as (2) above. On a regional basis, other LHG areas are associated with a contact in the Paleozoic rocks between clastic, confining unit rocks and the regional carbonate aquifer; however, the cause and nature of the LHG near Yucca Mountain is not evident. Another alternative explanation for the LHG is the presence of the Claim Canyon Caldera. The Claim Canyon Caldera is an area of extensive hydrothermal alteration that may result in a generalized reduction in permeability in the hydrogeologic units. Under this scenario, the edge of the Claim Canyon Caldera lies north of the Yucca Mountain repository and leads to the LHG where the caldera rocks have been hydrothermally altered.

There is no unequivocal explanation for the LHG. The presence of perched water at Boreholes USW G-2 and UE-25 WT\#6 has not been proven. As noted above, several explanations for the LHG have been proposed, some of which include perched water. The potentiometric-surface map presented in this report updates water levels in the region of the Nye County wells, but is an alternative interpretation of the LHG region as presented in USGS (2001 [DIRS 154625]). An expert elicitation panel (CRWMS 1998 [DIRS 100353], Section 3.2.2) concluded that the existence of the LHG or perched conditions does not impact the performance of Yucca Mountain. The panel suggested that to understand the cause, a borehole could be drilled and tested, which led to the drilling of Borehole USW WT-24. Drilling, testing, and monitoring of Borehole USW WT-24, (Graves 2001 [DIRS 155942]) indicated the existence of perched conditions and a regional water-table elevation of about $840 \mathrm{~m}$. After the water-bearing fracture was penetrated, the water level remained constant after the borehole was deepened by more than $100 \mathrm{~m}$, indicating the probability that the water level represents the regional potentiometric surface rather than another perched zone. However, because Borehole USW WT-24 is completed within the relatively low permeability Calico Hills Formation, as are Boreholes USW G-2 and UW-25 WT\#6, it cannot be ruled out that the 840-m water level in Borehole USW WT-24 could represent a second perched zone. Because the water encountered was from a fracture below a long interval of "dry rock," it may be more reasonable to conclude that the water level represents a regional potentiometric surface (connected by a network of water-bearing fractures within tight, dry rocks) rather than a second perched zone of saturated rocks. The perched and non-perched alternative interpretations were evaluated in the SZ site-scale flow model (BSC 2004 [DIRS 170037], Section 6.7) and found to yield similar flow fields.

The water level in Well NC-EWDP-7S is assumed to be perched in this report (see Section 5.1 and Table A-8). Water-level data in Well NE-EWDP-7SC located near Well NC-EWDP-7S provides an alternative interpretation of conditions at Well NC-EWDP-7S. The new water-level data from Nye County (DTN: MO0405NYE05819.215 [DIRS 170539]) for 2002 and 2003 indicates a downward hydraulic gradient with a water-level difference of about $8.5 \mathrm{~m}$ between zones 1 and 3, and a difference of nearly $34 \mathrm{~m}$ between zones 1 and 4 . Large downward gradients are observed between the deep and shallow monitored intervals at Borehole NC-EWDP-1DX (head difference of $38 \mathrm{~m}$ - see Table 6-4). The depth to water at both locations is anomalously shallow (7.7 $\mathrm{m}$ at $7 \mathrm{SC}$ and $16.9 \mathrm{~m}$ at $1 \mathrm{DX})$ and may represent locally perched conditions or the presence of a low permeability confining unit close to the surface that 
effectively impedes the downward migration of water to the more contiguous tuff and alluvium aquifers at greater depths.

The water level in the upper zone of Well NC-EWDP-7SC and in Well NC-EWDP-7S are about $830 \mathrm{~m}$, well above the measured water levels surrounding wells (see Figure 6-1). If these wells are not assumed perched, the impact to the potentiometric-surface map is a bull's eye contour at this location. This bull's eye could slightly modify the $775-\mathrm{m}$ and $750-\mathrm{m}$ contours nearby, but would not change the contours along the flow path from the repository to Fortymile Wash.

\subsection{UNCERTAINTY IN THE POTENTIOMETRIC-SURFACE ELEVATION MAP}

If any radionuclides escape from the Yucca Mountain repository and migrate to the SZ, they are likely to travel near the water table (within the upper several hundred meters of the SZ). There are several reasons this will occur: (1) there is a predominant upward hydraulic gradient along most of the predicted transport path, and (2) the most transmissive volcanic subunits of the Crater Flat formation (the Prow Pass and Bull Frog subunits) are located several hundred meters below the water table. The pathways near the water table were presented in the groundwater flow model (BSC 2004 [DIRS 170037], Figure 6-43) and indicate that flow paths will penetrate no more than about $300 \mathrm{~m}$ below the water table. An alternative model of the flow system yields flow paths that may be as deep as $600 \mathrm{~m}$ below the water table. The potentiometric-surface map presented on Figure 6-1 is intended to represent the potentiometric surface at or near the water table and is expected to be most representative of horizontal hydraulic gradients in the shallow portion of the SZ. The uncertainty in the potentiometric-surface map is directly related to the accuracy of the water-level measurements, other factors that influence water levels such as barometric fluctuations, and the underlying conceptual model that guides the contouring process.

Accuracy refers to the measurement of the water level and land surface elevation, both of which are needed to determine the water-level elevation. Other factors that may increase uncertainty refer to how well the measured water level represents the water levels near the top of the SZ. Several factors need to be considered: (1) water density variations, barometric and earth tide effects, and borehole deviation; (2) the vertical location of the open interval with respect to the shallow SZ; (3) temporal trends that would influence the final averaged water level; and (4) the extrapolation of contours lines to regions without measurements

\section{Measurement Accuracy}

The accuracy of the land surface elevation and the depth to water-level measurement are presented in Appendix A, Tables A-7 and A-8, respectively. The accuracy of the land surface (Table A-7) of the Nye County wells is listed as unknown. For the remainder of the wells, the accuracy of the land surface elevation for wells installed as part of the YMP is about $0.1 \mathrm{~m}$, although seven of the wells are given an unknown accuracy. For many of the private wells in the Amargosa Farms area, the accuracy of the land surface varies from $0.1 \mathrm{~m}$ to as large as $3.0 \mathrm{~m}$.

The accuracy of the depth to water-level measurements is presented in Table A-8. Savard (2001 [DIRS 165604], pp. 6 to 14) presents a discussion of the care that must be taken to minimize the measurement errors. The range in accuracy is from $0.01 \mathrm{ft}(0.003 \mathrm{~m})$ to the nearest foot $(0.3 \mathrm{~m})$. Excluding the Nye County wells, 13 measurement locations have a listed accuracy as unknown. 
Combining the uncertainty in the depth to water-level measurement and the land surface elevation, the accuracy of the water-level elevation ranges from $0.13 \mathrm{~m}$ to as large as $3.33 \mathrm{~m}$. The small measurement error cases generally apply to the wells drilled specifically for the YMP. The largest measurement errors occur in the Amargosa Farms area. The impact of this measurement error is assessed using the YMP wells in the vicinity of the repository where the combined error is generally about $0.13 \mathrm{~m}$. Even this small amount of measurement uncertainty could cause contour locations on Figure 6-1 to change. For example, one estimate of the hydraulic gradient in the vicinity of the repository is 0.00027 (based on water levels and coordinates of Wells USW G-4 and UE-25 WT \#13 given in Table A-1 of Appendix A). The horizontal hydraulic gradient is the water-level difference measured in two different wells divided by the horizontal distance between the two wells. Consider an example where the water-level measurement error of $0.13 \mathrm{~m}$ is the same in both wells (thus maintaining the gradient). The corresponding change in horizontal location of a particular potentiometric contour will be $480 \mathrm{~m}$, which is obtained by dividing the water-level change by the gradient $(0.13 \mathrm{~m} / 0.00027)$. If the error for each well is opposite in direction, the hydraulic gradient will change. In the example above, a $0.13 \mathrm{~m}$ error could increase the hydraulic gradient by 15 percent or decrease it by 21 percent. In this example, the larger uncertainty of $3.33 \mathrm{~m}$ was not used because it is not representative of the vast majority of well locations in the low gradient region.

The potential error in the contour location, due to measurement error, is inversely proportional to the hydraulic gradient. In a region of flat hydraulic gradient, the potential error is larger than in a region of steep hydraulic gradient. The impact of measurement error on the position of the potentiometric contour is also influenced by the number of nearby data points. A large change in the contour position (for example a nearly $1 \mathrm{~km}$ shift) is unlikely in a region where numerous water-level measurements are available to constrain the contour location because it is very unlikely that the measurement error in all the wells would be the same. However, in a portion of the study area where a contour location is based on one or two points, then the contour position is more uncertain. Fortunately, as can be seen on Figure 6-1, the region of shallow hydraulic gradient is also an area of numerous data points. Thus, it is reasonable to expect that measurement error will have a minor impact on the potentiometric contours on Figure 6-1.

\section{Other Factors}

The accuracy of measurement is only one of a number of factors that need to be considered. Even if measurements have zero error, the water levels may be influenced by various external factors such as barometric fluctuations and earth tides, water density variability, and borehole deviation. Fenelon (2000 [DIRS 160881], pp. 12 to 18) presents a careful discussion of these influences in the context of water levels measured on Pahute Mesa on the Nevada Test Site. In addition to these factors, the open interval and temporal trends in water levels also need to be considered.

\section{Barometric Fluctuations and Earth Tides}

Earth tides are diurnal fluctuations in water levels caused by the gravitation pull of the sun and moon. Barometric fluctuations are caused by successive weather systems that bring a variety of high and low pressure atmospheric conditions to an area. Both of these influences are most 
prominent in confined aquifers and are generally minimal (but not necessarily zero) in unconfined or water table wells. Fenelon (2000 [DIRS 160881], p. 14) presents evidence that the earth tide effect was about one order of magnitude smaller than the barometric effect in Well PM-2 on Pahute Mesa. In addition, there is no evidence that short-term variations in barometric pressure and earth tides cause long-term (multiyear) fluctuations in water level. For long-term records such as the data in this report that extend over a 10-year period, the averaging process is expected to remove the effect of barometric and earth tide fluctuations. However, for wells screened over a large thickness of the aquifer with a single data point, such as USW G-1 in Table A-2, the uncertainty in the measured value caused by barometric fluctuation increases. If the primary contributing interval in a well such as Well USW G-1 is a confined interval, then barometric fluctuations may increase uncertainty by an amount equal to the barometric efficiency of the well divided by the specific weight of water times the difference in barometric pressure at the time of measurement and the long-term average barometric pressure. The barometric efficiency of Well USW G-1 is unknown, but according to Freeze and Cherry (1979 [DIRS 101173], p. 234), the barometric efficiency typically falls in the range of 0.20 to 0.75 . If the barometric pressure on the day of measurement is 0.5 inches of mercury different from the long-term average barometric pressure, the error in water level could be as large as $0.13 \mathrm{~m}$ if the barometric efficiency is 0.75 . The deviation of barometric pressure of 0.5 inches of mercury falls within the range of barometric fluctuations as reported by the National Oceanic \& Atmospheric Administration for the Las Vegas Valley from 1937 to 2001 (http://www.wrh.noaa.gov/Lasvegas.climate/page34.html). The uncertainty for short duration water-level records due to barometric fluctuations is unlikely to be larger than $0.13 \mathrm{~m}$, which is similar in magnitude to the measurement error. As noted above, when other wells are nearby, the impact of uncertainty at one well location is diminished.

\section{Water Density (Water Temperature)}

Hydraulic head, as applied in most groundwater investigations, is the sum of an elevation head and a pressure head. The elevation head is usually defined as the elevation at the bottom of the open interval, referenced to mean sea level. The pressure head at the bottom of the open interval is the pressure divided by the specific gravity of water in the water column above. The specific gravity is defined as water density times the acceleration of gravity and it is typical to assume the acceleration of gravity is a constant. Fenelon (2000 [DIRS 160881], p. 11) notes that a number of factors may influence the density of water in the well including temperature, dissolved and suspended solids, nonaqueous phase liquids, compressibility of the water column, and gravity. Generally, temperature is the most important factor.

The comparison of hydraulic head in different boreholes may require consideration of differences in temperature of the water column. Consider an example with two wells, one screened over the first $50 \mathrm{~m}$ of an aquifer with a temperature of $30^{\circ} \mathrm{C}$ and a second screened over $500 \mathrm{~m}$ with temperature variation from $30^{\circ} \mathrm{C}$ at the water table to $40^{\circ} \mathrm{C}$ at the bottom. To compare the measured water levels, the water level in the deeper well needs to be corrected. Assuming the temperature distribution is linear with depth, the mean temperature in the deeper well is $35^{\circ} \mathrm{C}$. If the bottom of the deep well is the location of highest permeability, the full length of the water column is used for the correction. Fenelon (2000 [DIRS 160881], p. 12) provides an equation for correcting the water level by correcting the length of the water column above the bottom of the open interval. The corrected water column length is the product of the 
measured water column length times the ratio of the density at the measured temperature to the new or reference temperature. The water density at the reference temperature of $30^{\circ} \mathrm{C}$ is $0.99567 \mathrm{gm} / \mathrm{mL}$ and at the mean measured temperature of $35^{\circ} \mathrm{C}$ is $0.99406 \mathrm{gm} / \mathrm{mL}$ (Weast 1985 [DIRS 111561], p. F-10). Assuming the measured column length of $500 \mathrm{~m}$, the correction from a mean temperature of $35^{\circ} \mathrm{C}$ to a reference temperature of $30^{\circ} \mathrm{C}$ yields a new water column length of $499.19 \mathrm{~m}$. In this example, the deeper well water level would be lowered by about $0.8 \mathrm{~m}$ to be comparable to the shallow well. The magnitude of the correction is directly proportional to the length of the water column and the magnitude of the temperature differences. Longer columns and larger temperature differences result in larger corrections.

With respect to the potentiometric-surface map, the density changes as a result of temperature would be expected to be most important for wells with long open intervals such as USW G-1, USW G-2, and UE-25 J-13. A proper correction for density variation due to temperature requires a known temperature distribution and a clear idea of the interval, or intervals, that are most permeable and control the water level in the column. The density corrections may be complicated and can be difficult to quantify. This analysis points out the possible magnitude of error in a reported water level caused by temperature variations. This error may be important when comparing water levels measured in wells screened over different depth intervals. Five wells (USW G-1, USW G-3, USW H-4 upper, UE-25 J-13 and UE-25 b\#1 upper) have long open intervals that may contribute to larger errors in water level.

From the perspective of the vertical hydraulic head differences, the water levels from the deeper completion intervals are likely to exhibit larger uncertainties if the water levels are obtained as the depth to water in tubes open to the atmosphere and the completion interval. As noted in Table A-7 in Appendix A, most measurements in the vicinity of Yucca Mountain are made this way. If transducers are used to obtain pressure readings in sealed intervals, and pressure is measured directly, then the density correction is not needed. However, if the pressure is converted to hydraulic head, then temperature-dependent water density needs to be taken into account. This is the case for seven of the Nye County EWDP wells (see Table A-7).

\section{Borehole Deviation}

Boreholes are rarely perfectly vertical. If the borehole is not vertical, the measured depth to water is larger than the true vertical depth to water. This will result in a calculated water-level elevation that is too low. Fenelon (2000 [DIRS 160881], p. 10) found that typical borehole deviation corrections for wells on Pahute Mesa on the Nevada Test Site can range from 0 to $0.5 \mathrm{ft}(0.15 \mathrm{~m})$. Savard (2001 [DIRS 165604], pp. 11 to 12) calculated a larger range of 0.012 to $0.482 \mathrm{~m}$ for wells and boreholes associated with the YMP. Savard (2001 [DIRS 165604], p. 12) notes that borehole deviation corrections are performed on all wells for which gyroscopic surveys are available. Only Boreholes UE-25 J-11, UE-25 J-12, UE-25 J-13, UE-29 a \#1, and UE-29 UZN \#91 have no correction for borehole deviation. Borehole deviation does not appear to be a significant source of error in the potentiometric-surface map on Figure 6-1.

\section{Averaging Process}

The water-level elevation used in the potentiometric-surface analysis is the average of water-levels collected over a long period of time. The averaging process will generally average 
out the periodic processes such as barometric fluctuation and earth tides. However, care must be taken when averaging water-level records that may have a temporal trend. One way to examine temporal trends is to examine the standard deviation of the water-level measurements. Graves et al. (1997 [DIRS 101046], Table 2) present the mean and standard deviation of water-level measurements from 36 open intervals in 28 wells. With the exception of the lower interval of Well USW H-3, most of the wells show a relatively small standard deviation. The 95-percentile confidence interval for the mean water level is estimated using the relationship 1.96 times the standard deviation divided by the square root of the number of data points. The largest 95 percent confidence interval about the mean water level was $0.1 \mathrm{~m}$. The majority of wells had a 95 percent confidence interval of less than $0.03 \mathrm{~m}$. It appears that temporal trends in water levels do not contribute significant uncertainty to the potentiometric-surface map, if enough data are available to calculate a long-term average. However, for a single data point, greater uncertainty exists because an average value cannot be calculated. Savard (2001 [DIRS 165604]) presents annual average water-level values through 1999. In only two cases (not associated with packer failure) have temporal trends been noted. These were the deep completions in Well USW H-1 (tubes 1 and 2). All the shallow completions used to construct Figure 6-1 show small temporal fluctuations. Therefore, uncertainties caused by temporal variations are quite small.

\section{Data from New Wells}

To this point, the discussion has focused on sources of uncertainty related to existing wells and boreholes. Another key factor is the uneven distribution of water-level measuring points. In areas of the map (Figure 6-1) where data are sparse, the potentially large uncertainty in the potentiometric-surface contours is indicated by dashed contour lines. The uncertainty caused by interpolation (and often extrapolation) cannot be quantified unless new water-level observation wells are drilled. Fortunately, several new wells near the Fortymile Wash allow for an assessment of uncertainty along a portion of the flow path from the repository to the accessible environment.

On Figure 6-1, there is a large gap of nearly $10 \mathrm{~km}$ between observed water levels in Well UE-25 J-12 and the Nye County wells along U.S. Highway 95 (See Figure 1-2 for the well locations). Two contour lines $(720 \mathrm{~m}$ and $725 \mathrm{~m})$ are interpolated into that region. Twelve new wells were installed at eight locations as part of the Phases III and IV drilling for Nye County. The new well locations, land surface elevation, depth to water, and data source are presented in Table 6-5. The new well locations are shown on Figure 1-2. The associated new water levels are shown on Figure 6-2 along with the water-level contours from Figure 6-1 and an example of how those contours might change if the EWDP Phases III and IV data were added. Figure 6-2 is provided for use in this report only. It is not intended to be used by any downstream analyses and no output DTN has been generated. With the exception of Boreholes NC-EWDP-19M1 and NC-EWDP-19M2, the new wells fill gaps in the existing spatial distribution of data. The data from the Phases III and IV wells (DTN: MO0405NYE05819.215 [DIRS 170539]) is unqualified, but can be used to assess the magnitude of this uncertainty, at least in the vicinity of Fortymile Wash. Assessing the contours in Fortymile Wash, based on these new data, suggests the $720-\mathrm{m}$ and $725-\mathrm{m}$ contours in Fortymile Wash would be shifted to the south about $1.5 \mathrm{~km}$. This shift to the south will steepen the hydraulic gradient in the alluvium in the region of Fortymile Wash, just north of U.S. Highway 95 by about 30 percent (using the $725-\mathrm{m}$ contour 
and the Washburn Well to calculate the gradient), and reduce the gradient south of Well UE-25 $\mathrm{J}-12$ by about 50 percent (using Well $\mathrm{J}-12$ and the $725-\mathrm{m}$ contour).

Table 6-5. Water Levels for the Time Period of January 2003 to August 2003 Used for Assessment of New Data

\begin{tabular}{|c|c|c|c|c|c|c|}
\hline $\begin{array}{c}\begin{array}{c}\text { Date of Water } \\
\text { Level } \\
\text { Measurement }\end{array} \\
\end{array}$ & Site Name & $\begin{array}{c}\text { Easting } \\
\text { (UTM) }\end{array}$ & $\begin{array}{l}\text { Northing } \\
\text { (UTM) }\end{array}$ & \begin{tabular}{|c|}
$\begin{array}{c}\text { Land-Surface } \\
\text { Altitude } \\
\text { (meters) }\end{array}$ \\
\end{tabular} & $\begin{array}{c}\text { Mean Water- } \\
\text { level Altitude } \\
\text { (meters) }\end{array}$ & Source \\
\hline $5 / 15 / 2003$ & $\begin{array}{l}\text { NC-EWDP-10P, } \\
\text { shallow }\end{array}$ & 553149 & 4064916 & 903.6 & 727.0 & $\begin{array}{l}\text { MO0405NYE05819.215 } \\
\text { MO0206GSC02074.000 }\end{array}$ \\
\hline $5 / 15 / 2003$ & $\begin{array}{l}\text { NC-EWDP-10P, } \\
\text { deep }\end{array}$ & 553149 & 4064916 & 903.6 & 726.9 & $\begin{array}{l}\text { MO0405NYE05819.215 } \\
\text { MO0206GSC02074.000 }\end{array}$ \\
\hline 6/10/2003 & $\begin{array}{l}\text { NC-EWDP-10S, } \\
\text { probe } 1\end{array}$ & 553140 & 4064899 & 903.3 & 727.0 & $\begin{array}{l}\text { MO0405NYE05819.215 } \\
\text { MO0203GSC02034.000 }\end{array}$ \\
\hline 6/10/2003 & $\begin{array}{l}\text { NC-EWDP-10S, } \\
\text { probe } 2\end{array}$ & 553140 & 4064899 & 903.3 & 727.0 & $\begin{array}{l}\text { MO0405NYE05819.215 } \\
\text { MO0203GSC02034.000 }\end{array}$ \\
\hline $5 / 15 / 2003$ & NC-EWDP-18P & 549416 & 4067233 & 964.5 & 727.5 & $\begin{array}{l}\text { MO0405NYE05819.215 } \\
\text { MO0203GSC02034.000 }\end{array}$ \\
\hline $7 / 10 / 2003$ & $\begin{array}{l}\text { NC-EWDP-19IM1, } \\
\text { probe } 1\end{array}$ & 549317 & 4058290 & 819.1 & 710.2 & $\begin{array}{l}\text { MO0405NYE05819.215 } \\
\text { MO0206GSC02074.000 }\end{array}$ \\
\hline $7 / 10 / 2003$ & $\begin{array}{l}\text { NC-EWDP-19IM1, } \\
\text { probe } 2\end{array}$ & 549317 & 4058290 & 819.1 & 710.6 & $\begin{array}{l}\text { MO0405NYE05819.215 } \\
\text { MO0206GSC02074.000 }\end{array}$ \\
\hline $7 / 10 / 2003$ & $\begin{array}{l}\text { NC-EWDP-19IM1, } \\
\text { probe } 3\end{array}$ & 549317 & 4058290 & 819.1 & 710.9 & $\begin{array}{l}\text { MO0405NYE05819.215 } \\
\text { MO0206GSC02074.000 }\end{array}$ \\
\hline 7/9/2003 & $\begin{array}{l}\text { NC-EWDP-19IM1, } \\
\text { probe } 4\end{array}$ & 549317 & 4058290 & 819.1 & 711.6 & $\begin{array}{l}\text { MO0405NYE05819.215 } \\
\text { MO0206GSC02074.000 }\end{array}$ \\
\hline 7/9/2003 & $\begin{array}{l}\text { NC-EWDP-19IM1, } \\
\text { probe } 5\end{array}$ & 549317 & 4058290 & 819.1 & 712.4 & $\begin{array}{l}\text { MO0405NYE05819.215 } \\
\text { MO0206GSC02074.000 }\end{array}$ \\
\hline $5 / 15 / 2003$ & NC-EWDP-19M2 & 549337 & 4058291 & 819.3 & 711.7 & $\begin{array}{l}\text { MO0405NYE05819.215 } \\
\text { MO0206GSC02074.000 }\end{array}$ \\
\hline $5 / 15 / 2003$ & $\begin{array}{l}\text { NC-EWDP-22PA, } \\
\text { shallow }\end{array}$ & 552020 & 4062038 & 868.6 & 724.9 & $\begin{array}{l}\text { MO0405NYE05819.215 } \\
\text { MO0206GSC02074.000 }\end{array}$ \\
\hline $5 / 15 / 2003$ & $\begin{array}{l}\text { NC-EWDP-22PA, } \\
\text { deep }\end{array}$ & 552020 & 4062038 & 868.6 & 724.8 & $\begin{array}{l}\text { MO0405NYE05819.215 } \\
\text { MO0206GSC02074.000 }\end{array}$ \\
\hline $5 / 15 / 2003$ & $\begin{array}{l}\text { NC-EWDP-22PB, } \\
\text { shallow }\end{array}$ & 552038 & 4062037 & 868.5 & 724.8 & $\begin{array}{l}\text { MO0405NYE05819.215 } \\
\text { MO0206GSC02074.000 }\end{array}$ \\
\hline $5 / 15 / 2003$ & $\begin{array}{l}\text { NC-EWDP-22PB, } \\
\text { deep }\end{array}$ & 552038 & 4062037 & 868.5 & 724.8 & $\begin{array}{l}\text { MO0405NYE05819.215 } \\
\text { MO0206GSC02074.000 }\end{array}$ \\
\hline $6 / 17 / 2003$ & $\begin{array}{l}\text { NC-EWDP-22S, } \\
\text { probe } 1\end{array}$ & 552019 & 4062020 & 868.4 & 724.9 & $\begin{array}{l}\text { MO0405NYE05819.215 } \\
\text { MO0203GSC02034.000 }\end{array}$ \\
\hline $6 / 17 / 2003$ & $\begin{array}{l}\text { NC-EWDP-22S, } \\
\text { probe } 2\end{array}$ & 552019 & 4062020 & 868.4 & 724.9 & $\begin{array}{l}\text { MO0405NYE05819.215 } \\
\text { MO0203GSC02034.000 }\end{array}$ \\
\hline 6/17/2003 & $\begin{array}{l}\text { NC-EWDP-22S, } \\
\text { probe } 3\end{array}$ & 552019 & 4062020 & 868.4 & 724.9 & $\begin{array}{l}\text { MO0405NYE05819.215 } \\
\text { MO0203GSC02034.000 }\end{array}$ \\
\hline $6 / 17 / 2003$ & $\begin{array}{l}\text { NC-EWDP-22S, } \\
\text { probe } 4\end{array}$ & 552019 & 4062020 & 868.4 & 724.9 & $\begin{array}{l}\text { MO0405NYE05819.215 } \\
\text { MO0203GSC02034.000 }\end{array}$ \\
\hline
\end{tabular}


Table 6-5. Water Levels for the Time Period of January 2003 to August 2003 Used for Assessment of New Data (Continued)

\begin{tabular}{|c|l|c|c|c|c|c|}
\hline $\begin{array}{c}\text { Date of Water } \\
\text { Level } \\
\text { Measurement }\end{array}$ & \multicolumn{1}{|c|}{ Site Name } & $\begin{array}{c}\text { Easting } \\
\text { (UTM) }\end{array}$ & $\begin{array}{c}\text { Northing } \\
\text { (UTM) }\end{array}$ & $\begin{array}{c}\text { Land-Surface } \\
\text { Altitude } \\
\text { (meters) }\end{array}$ & $\begin{array}{c}\text { Mean Water- } \\
\text { level Altitude } \\
\text { (meters) }\end{array}$ & Source \\
\hline $5 / 15 / 2003$ & $\begin{array}{l}\text { NC-EWDP-23P, } \\
\text { shallow }\end{array}$ & 553924 & 4059875 & 853.5 & 724.3 & $\begin{array}{l}\text { MO0405NYE05819.215 } \\
\text { MO0206GSC02074.000 }\end{array}$ \\
\hline $5 / 15 / 2003$ & $\begin{array}{l}\text { NC-EWDP-23P, } \\
\text { deep }\end{array}$ & 553924 & 4059875 & 853.5 & 724.3 & $\begin{array}{l}\text { MO0405NYE05819.215 } \\
\text { MO0206GSC02074.000 }\end{array}$ \\
\hline $5 / 14 / 2003$ & NC-EWDP-16P & 545665 & 4064263 & $880.6^{a}$ & 729.4 & $\begin{array}{l}\text { MO0405NYE05819.215 } \\
\text { MO0307GSC03094.000 }\end{array}$ \\
\hline $5 / 14 / 2003$ & NC-EWDP-27P & 544935 & 4065275 & $906.4^{a}$ & 728.6 & $\begin{array}{l}\text { MO0405NYE05819.215 } \\
\text { MO0307GSC03094.000 }\end{array}$ \\
\hline $5 / 14 / 2003$ & NC-EWDP-28P & 545746 & 4062393 & $843.5^{a}$ & 729.3 & $\begin{array}{l}\text { MO0405NYE05819.215 } \\
\text { MO0307GSC03094.000 }\end{array}$ \\
\hline
\end{tabular}

${ }^{a}$ Slab Elevation (not ground surface elevation).

To the west, the three new wells (NC-EWDP-16P, 27P, and 28P) have lower water levels than were estimated during the creation of Figure 6-1. To account for the new data, part of the $730 \mathrm{~m}$ contour line would be moved to the west, almost as far as the Solitario Canyon fault. The new 730-m contour line is more complex. In the south along U.S. Highway 95, a small region of higher water level extends from southern crater flat along U.S. Highway 95 toward Fortymile Wash. If the water level in Well NC-EWDP-7S is not considered perched, then a higher water-table mound could be drawn centered on Well NC-EWDP-7S and extending to the east. The assumption of perched water at Well NC-EWDP-7S does not alter the location of the revised 730-m contour on Figure 6-2. North of the three new wells, the revised contour creates a more southerly hydraulic gradient near the southern end of Yucca Mountain. Finally, for the most part, the distance between the 730- and 725-m lines has increased for the area south and southeast of Yucca Mountain. This increased distance leads to a decrease in the hydraulic gradient in this region.

This analysis suggests that the hydraulic gradient in the volcanic units beneath southern Fortymile Wash may be 50 percent less than initially estimated. In the alluvium near the accessible environment, the hydraulic gradient may be 30 percent larger than initially estimated from the interpolated contours on Figure 6-1. If hydraulic conductivity is unchanged, then the changes in the hydraulic gradient translate directly to changes in groundwater-specific discharge. Specific discharge can be calculated as the product of hydraulic conductivity and hydraulic gradient (Freeze and Cherry 1979 [DIRS 101173], p. 16). A 50 percent decrease in hydraulic gradient that reflects a hydraulic conductivity increase of 50 percent will produce no change in the specific discharge. With specific discharge 50 percent smaller along a large portion of the flow path along Fortymile Wash, the radionuclide transport times would be expected to be twice as long through that same region. Conversely, in the alluvium near U.S. Highway 95, the specific discharge could be 30 percent larger leading to a decrease in transport time in that region. On the other hand, the changes in hydraulic gradient resulting from the new well data may simply reflect heterogeneity in the hydraulic conductivity. Perhaps the hydraulic conductivity in the mid reaches of Fortymile Wash is 50 percent larger and the conductivity of the alluvium in the area of U.S. Highway 95 is 30 percent smaller than used in the calibrated 
flow model (BSC 2004 [DIRS 170037]). In that case, the updated hydraulic gradients would simply reflect the differences in hydraulic conductivity between the model and reality, but the specific discharge is unchanged.

The uncertainty in the specific discharge, as indicated by the revised water-level contours, is much less than the range of uncertainty currently used in the SZ flow and transport model abstraction (BSC 2004 [DIRS 170042]). The uncertainty in specific discharge is implemented by a multiplication factor in the abstraction model (BSC 2004 [DIRS 170042], Figure 6-15) where the base case specific discharge is assigned a multiplication factor of 1.0. The specific discharge multiplication factor is characterized by a cumulative probability density function with three probability regions. There is: 1) a 10 percent probability the specific discharge lies between $1 / 30$ and $1 / 3$ of the calibrated specific discharge from the SZ site-scale flow model (BSC 2004 [DIRS 170037]); 2) an 80 percent chance it lies between 1/3 and 3 times the calibrated value; and 3) a 10 percent chance it lies between 3 and 10 times the calibrated value. The uncertainty in the potentiometric surface due to data gaps was shown to be in the range of 30 percent to 50 percent in the Fortymile Wash region near U.S. Highway 95. This magnitude of uncertain is much less than the total range of uncertainty currently included in the groundwater flow and transport abstraction model (BSC 2004 [DIRS 170042]). In addition, the uncertainty in the specific discharge from the change in contours lies within the range of $1 / 3$ to 3 times the calibrated value where 80 percent of the realizations are expected to occur.

The impact to the groundwater flow paths from the EWDP Phase III and IV well data is expected to be small. The modified contour lines would be consistent with more southerly flow paths, from the southern portions of the Yucca Mountain repository. In the vicinity of Fortymile Wash, the convergence of the flow lines would not change as a result of the relatively small shift in the contours. In all cases, the hydraulic gradient south and southeast of Yucca Mountain is smaller than on Figure 6-1.

\section{Open Interval}

To be representative of the shallow hydraulic head distribution, the open interval of the wells should be representative of the interval near the top of the aquifer. Table A-5 in Appendix A includes a listing of the top and bottom of the open interval for each well. Several wells have multiple completions that have been used to assess vertical gradients. Additionally, most wells are open at or near the water table. The length of the open interval varies greatly from well to well. Some wells are open to relatively narrow intervals such as UE-25 WT \#14 where the length of the open interval is $52 \mathrm{~m}$. Other wells are open over much longer intervals, such as USW G-3, with an open interval of $741 \mathrm{~m}$. The longer screened wells (USW G-1, USW G-3, UE-25 J-13, USW H-4, USW G-4, USW H-3, USW H-6, UE 25 a\#1, UE 25 b\#1, UE 25 c\#1, UE $25 \mathrm{c} \# 2$, and UE $25 \mathrm{c \# 3}$ ) are scattered throughout, or near, the low hydraulic gradient region and provide the opportunity to measure composite water levels and perhaps the ability to measure vertical flow caused by vertical hydraulic gradients. However, wells with longer open intervals add additional uncertainty to the potentiometric-surface map.

As noted above, uncertainty in the water level is increased because of water temperature effects. Additionally, if there is vertical variation of hydraulic head with depth, the measured depth to water in the long-open interval wells may not represent the water table, but rather the hydraulic 
head at some other depth in the aquifer. The composite water level in a long-screened well is representative of the most permeable zones in the well. If the most permeable zone is near the water table, then the water level in the long open interval well will likely be representative of the water table. However, if the most permeable zone is deeper in the well, then the water level in the well will be representative of the deep permeable zone. Without knowledge of the permeability distribution with depth, and the hydraulic head distribution with depth, it is difficult to quantify this uncertainty. Of the wells noted above, only USW G-1 and USW H-4 are located in close proximity to other wells. This proximity provides information to assess the potential errors.

Consider Well USW G-1, with Wells USW UZ-14 and USW H-1 nearby. Well USW G-1 is open from $754.2 \mathrm{~m}$ to $-503 \mathrm{~m}$ with a water level of $754.2 \mathrm{~m}$. Well USW UZ-14 lies to the northwest of Well USW G-1, and is open near the water table and has a high water level of $779.0 \mathrm{~m}$. To the east of Well USW G-1, Well USW H-1 is completed in four intervals, the shallow interval of $730 \mathrm{~m}$ to $630 \mathrm{~m}$ has a water level of $730.8 \mathrm{~m}$ while the deepest interval of $-480 \mathrm{~m}$ to $-511 \mathrm{~m}$ has a water level of $785.5 \mathrm{~m}$. The measured water level in Well USW G-1 appears to be in a transition zone about midway between Well USW UZ-14 and the shallow interval of Well USW H-1. However, the 754.2-m value measured in Well USW G-1 is also about midway between the measured water levels in the deep and shallow completions of Well USW H-1. The long open interval of Well USW G-1 may be a composite water level in a region where water level varies with depth and may not representative of the water table. It is possible that the water level measured in Well USW G-1 may misrepresent the water table elevation by as much as $20 \mathrm{~m}$ or $25 \mathrm{~m}$. Even if that is the case, the position of Well USW G-1 with respect to the nearby wells suggests that the influence on the potentiometric-surface contours would be very small because the water levels in Wells USW UZ-14 and USW H-1 are reliable. If the true water table elevation at location Well USW G-1 is actually closer to $730 \mathrm{~m}$ rather than the measured composite level of $754.2 \mathrm{~m}$, the $750 \mathrm{~m}$ contour would shift to the west or northwest by perhaps a few hundred meters. This magnitude change is small considering the scale of the map on Figure 6-1.

The other example is Well USW H-4 with nearby Wells USW SD-12, USW WT-2, and USW SD-7. Of the two completions in Well USW H-4, the shallow is open from $730.4 \mathrm{~m}$ to $60.5 \mathrm{~m}$ with a water level of $730.4 \mathrm{~m}$ and the deep completion is open from $60.5 \mathrm{~m}$ to $29.5 \mathrm{~m}$ with a water level of 730.5. The other wells are all completed near the water table and have measured water levels that range from $727.6 \mathrm{~m}$ to $730.6 \mathrm{~m}$. Recall that Wells USW SD-12 and Well USW SD-7 have few measurements and are considered less reliable (see Tables A-2 and A-3 of Appendix A). However, the similarity of the water levels in all these wells suggests that the water level measured in Well USW H-4 is probably not influenced by the long open interval because there does not appear to be much of a difference in water level with depth in any of the nearby wells.

The impact of different open intervals appears to be relatively small for several reasons. In the case of Well USW G-1, an error of as large as $20 \mathrm{~m}$ would impact the potentiometric-surface contour by a small amount because of control provided by other nearby wells. At Well USW H-4, there does not appear to be a significant difference in hydraulic head with depth, thus there would be little impact of the longer open interval from permeability or water-level fluctuations with depth. For the other wells, the fact that measured water levels are consistent 
with other water levels in the area suggests that if errors in the water levels are due to the long open interval, the errors must be small (perhaps $1 \mathrm{~m}$ or less), or large discontinuities would be observed.

\section{Conceptual Model Uncertainty}

The final component to uncertainty in the potentiometric-surface map is the conceptual model. The conceptual model defines the intent of the map and thus controls how data will be interpreted and is tied to the assumptions presented in Section 5. The potentiometric map is intended to represent:

- The average water-level conditions that existed in the early $1990 \mathrm{~s}$

- The water levels in the shallow portion of the aquifer and not depth-averaged conditions

- The regional potentiometric surface and not perched water

- The role of faults - primarily as barriers to flow.

Each of these will be discussed in turn. The selection of a time period eliminates the need to approximate predevelopment water levels. The assumption is made that current conditions will continue into the future, thus pumping effects in the southern portion of the model area are included in the water levels that are lower than predevelopment levels. Water levels collected from periods of time other than the early 1990s may introduce uncertainty into the potentiometric-surface map. For example, the water-level data obtained from the Nye County wells through December 2000 may introduce errors if water levels in the region are changing. Fenelon and Moreo (2002 [DIRS 164662]) examine water-level trends in the Yucca Mountain region from 1960 to 2000. For two wells located near the community of Amargosa Valley (near the intersection of U.S. Highway 95 and Nevada State Highway 373), a downward trend in water level of 1 foot $(0.3 \mathrm{~m})$ from 1992 to 2000 was observed in the Airport Well (Fenelon and Moreo 2000 [DIRS 164662], Table 6 site AD-2). A second well, the NDOT well (Fenelon and Moreo 2002 [DIRS 164662], Table 6, site AD-2a) does not exhibit a trend in water levels, but the variability in measured levels in that well spans a range of about 2 feet $(0.61 \mathrm{~m})$. Trends in other wells, such as UE-25 J-12 and UE-25 J-13 produce water-level changes of less than $0.5 \mathrm{ft}$ $(0.15 \mathrm{~m})$ over the time frame of 1992 to 2000 . It would appear that using a reference time of the early 1990 s does not introduce significant uncertainty into the potentiometric map.

The potentiometric-surface map is intended to represent the shallow portion of the flow system, but not perched water. From the data in Table 6-4, it is clear that vertical differences in hydraulic head exist in the Yucca Mountain vicinity. Excluding the Nye County wells for the moment, the hydraulic head variation with depth ranges from $0.1 \mathrm{~m}$ in Well USW H-4 to as much as $54.7 \mathrm{~m}$ in Well USW H-1. Two wells with long open intervals, USW G-1 and USW G-3 are both located in proximity to other wells with observed vertical head differences. The data from Wells USW G-1 and USW G-3 should be viewed skeptically because they may not be representative of the conditions in the shallow portion of the flow system. As noted in the previous sections, the error at Well USW G-1 could be as large as $20 \mathrm{~m}$, but the impact of errors at Well USW G-1 on the potentiometric contours are expected to be very small because of the constraints provided by nearby wells. The similarity of water levels at Well USW G-3 and other nearby wells is an indication that the errors at Well USW G-3 are likely not as large as at Well USW G-1. 


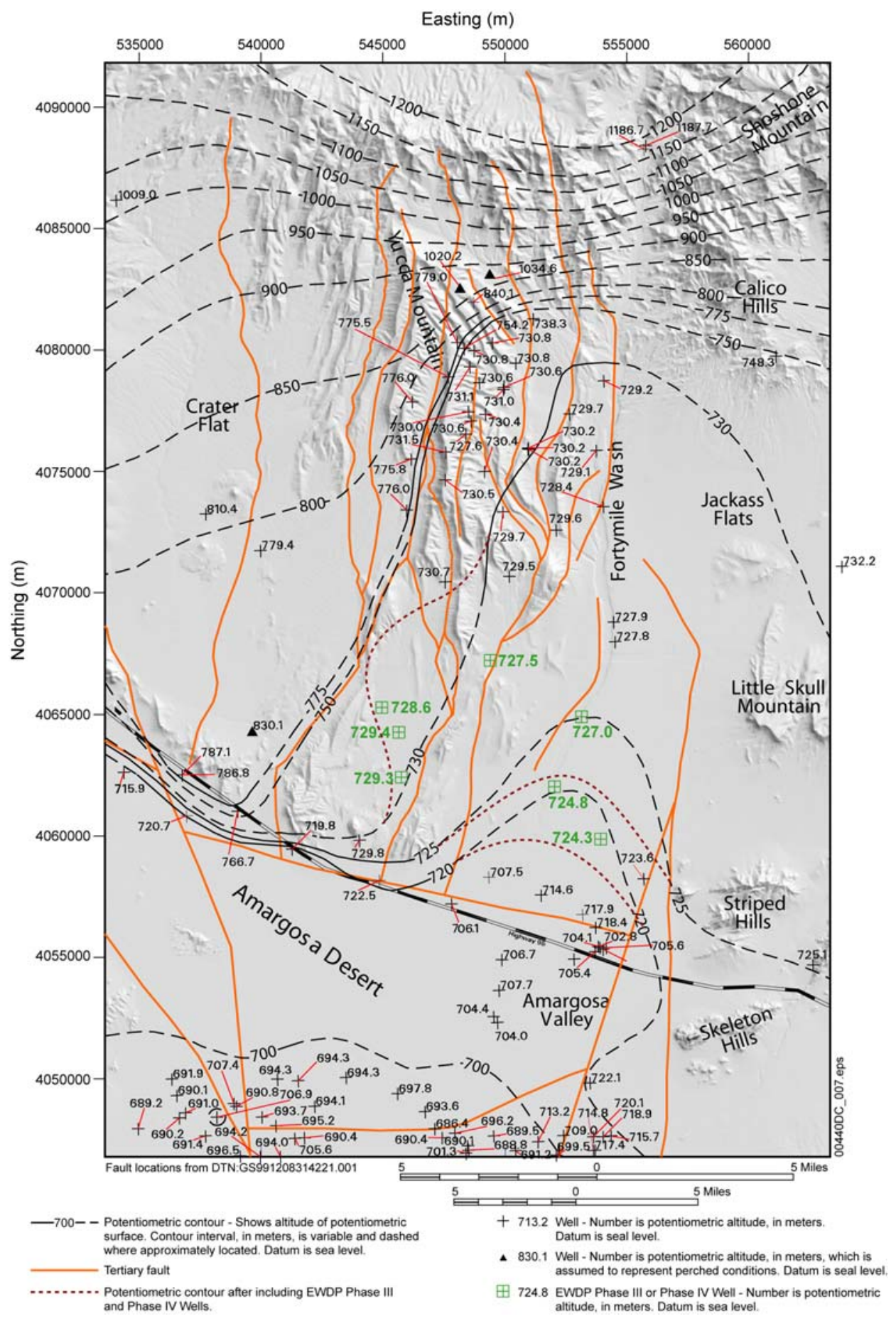

DTN: $\quad$ MO0409SEPPSMPC.000 [OUTPUT].

Sources: USGS (2004 [DIRS168473]), DTNs: GS010608312332.001, MO0203GSC02034.000 [DIRS 168375], MO0206GSC02074.000 [DIRS 168378], MO0307GSC03094.000 [DIRS 170556].

Figure 6-2. Revised Potentiometric-Surface Map Showing Possible Changes After Including EWDP Phases III and IV Wells 
Data from the Nye County wells can be grouped into two distinct regions. The data from wells in Fortymile Wash near U.S. Highway 95 indicate upward hydraulic gradients while the wells west of Fortymile Wash generally show a downward hydraulic gradient. The potentiometric surface in the vicinity of U.S. Highway 95 is represented by data from open intervals in the shallow portions of the aquifer.

Several wells in the region, including Wells USW G-2 and UE-25 WT \#6, are assumed to represent perched water conditions and are excluded from the potentiometric surface contouring. The assumption of perched water has a significant impact on the potentiometric surface. Consider Well UE-25 WT \#6, where the measured water level is about $160 \mathrm{~m}$ above the expected regional potentiometric surface depicted on Figure 6-1. The potentiometric surface would be very different north of the repository location if the perched water levels were treated as part of the regional flow system.

As noted in Section 5.2, faults may impact the flow of groundwater and hence also impact the contouring of the potentiometric surface on Figure 6-1. Faults may have no impact to flow or act as barriers to flow because of fault gouge or the juxtaposition of low permeability rocks against high permeability rocks. Faults also create zones of fracturing that may act as conduits for groundwater flow. As noted in Section 5.2, Assumption 2, some faults appear to exhibit both characteristics; acting as a barrier to flow across the fault, but as a conduit to flow along the fault. No consistent assumption regarding the role of faults was applied to the contour mapping. There are several reasons for this: (1) The hydraulic properties of individual faults are generally not known; (2) the orientation of the fault with respect to the regional flow system may heighten or lessen the impact; and (3) the distribution of data over the area limits the areas where the impact of faults would be apparent.

The hydraulic properties of individual faults are not measured. If they were, it is likely that the hydraulic conductivity would be anisotropic, with different hydraulic conductivity values perpendicular and parallel to the fault. Additionally, the properties would likely be heterogeneous, thus, even if measurements were available for selected locations along some faults, extrapolation of those properties to the entire length of the fault or to other faults would be difficult.

The orientation of a fault, regardless of its properties, may cause the fault to have no impact to the potentiometric surface. Consider a fault with hydraulic properties of a barrier, oriented such that the strike of the fault is parallel to the regional direction of flow. Water would flow parallel to the fault and no impact to the potentiometric surface would be observed. Similarly, a permeable fault oriented perpendicular to flow would also have a minimal impact on the potentiometric surface. Thus, faults of most concern would be barriers oriented perpendicular to flow and permeable faults oriented parallel to flow. Faults acting as barriers have been represented as steep water-level gradient zones along Solitario Canyon and U.S. Highway 95, south of Crater Flat, to account for the higher water levels in Crater Flat, yet reproduce the relatively flat potentiometric surface east of Solitario Canyon and in the Amargosa Desert. Other faults, especially those east of the Yucca Mountain crest, are not depicted as influencing the potentiometric-surface contours. This is not to suggest that these faults have no impact, rather it indicates the impact is not readily apparent given the magnitude of water levels and the density of data points. 
Another factor that will add uncertainty to the potentiometric-surface contours is the uncertainty in the assignment and juxtaposition of the underlying hydrogeologic units, as well as positioning and depth of faults. The extrapolation of water levels to areas without measurements relies on professional judgment, which includes assessing the material properties of the hydrogeologic units as they affect flow. Identification of the hydrogeologic rock types, fault extent, and depths is based on the hydrogeologic framework model (HFM), which is subject to interpretation and interpolation where data are not available. It is reasonable to expect the potentiometric-surface contours to have greater uncertainty in regions where the underlying HFM is also uncertain. One area of HFM uncertainty is the high-gradient region to the north, because there is not enough data to fully constrain the HFM in that region.

These alternative conceptual models of the hydrogeologic system have led to the creation of alternative potentiometric-surface maps. To assess the importance of these alternatives, the SZ site-scale flow model (BSC 2004 [DIRS 170037], Section 6.7) simulated the groundwater flow fields with different configurations of the potentiometric surface. Thus, the uncertainty due to different conceptual models has been incorporated in the SZ site-scale flow model.

\section{Summary of Potential Errors}

Table 6-6 presents a summary of the potential errors or corrections to water levels that may impact the water levels presented on Figure 6-1. The minimum error is on the order of $0.1 \mathrm{~m}$. This represents a vertical well with careful measurements, surveyed wellhead elevation, short-screened interval near the water table, and a long record with no temporal trend. The other extreme is a deviated, long open interval well, with few water-level measurements, a temperature variation with depth, and a temporal trend. In this case, the error in water level could be as large as $1.61 \mathrm{~m}$, or more, depending on the magnitude of the temperature correction and how well the composite water level represents the shallow portion of the aquifer.

Table 6-6. Summary of Potential Uncertainties Impacting the Potentiometric-Surface Map

\begin{tabular}{|l|c|c|}
\hline \multicolumn{1}{|c|}{ Type of Error } & Minimum (meters) & Maximum (meters) \\
\hline Measurement Error & 0.1 & 0.33 \\
\hline Barometric and Earth Tides & 0 & 0.13 \\
\hline Water Temperature & 0 & 0.8 or more \\
\hline Borehole Deviation & 0 & 0.15 \\
\hline Temporal Trends & 0.01 & 0.1 \\
\hline Total & 0.11 & 1.51 or more \\
\hline
\end{tabular}

\section{Implications of Uncertainty for the Potentiometric-surface Map}

A number of uncertainties have been identified that might impact the potentiometric-surface map presented on Figure 6-1. Items noted in Table 6-6 (measurement error, barometric and earth tide effects, water density, borehole deviation, and temporal trends) may introduce errors in water level from $0.1 \mathrm{~m}$ to more than $1 \mathrm{~m}$. Errors of this magnitude will have a negligible effect in areas west of Solitario Canyon, north of the Yucca Mountain site, Jackass Flats, and south of U.S. Highway 95. These potential errors, however, would be much more important in the low-gradient area east of the Yucca Mountain crest and into Fortymile Wash. Water-level 
contours could shift laterally up to several kilometers if the water levels were adjusted as much as $1 \mathrm{~m}$ up or down in several key wells. Even so, the general trend of higher water levels west of Solitario Canyon and lower water levels in Fortymile Wash would remain. Thus, the observation of flow from the repository to Fortymile Wash remains. Another key point is that in the low gradient area of the repository, water-level differences from well to well are typically only a few tenths of a meter. Given the uncertainties, it is not defensible to draw detailed resolution contours in the low gradient region in an attempt to match each of the individual water levels. Doing so would lead to local scale (on the order of 1 or $2 \mathrm{~km}$ ) variations in the direction of the hydraulic gradient that may have no basis in reality.

Other factors that may contribute more uncertainty include the long interval completions that may be measuring water levels at an unknown depth in the well. In a long completion, the composite water level represents the most permeable zone or zones in the well. The permeable zones may not necessarily occur at the shallow depths represented by the contours on Figure 6-1. Locations with significant hydraulic head differences with depth occur at several locations in the low gradient region. This suggests that measured hydraulic head in long open interval wells should be used with caution.

\section{Discussion of Alternative Potentiometric-Surface Maps}

The potentiometric-surface map presented by Czarnecki et al. (1997 [DIRS 141643], Figure 5) is identical in areal extent to the potentiometric-surface map developed for this revision (Figure 6-1). Examination of that potentiometric-surface map illustrates an alternative interpretation constructed from similar water-level data. Differences in the two maps occur at the boundaries of the maps, where there are little or no data; and where the potentiometric surface is influenced by major faults. The major difference in the shape of the potentiometric contours occurs in the northern and northwestern area of the maps. Czarnecki et al. (1997 [DIRS 141643]) suggest a closing of the contour lines to the north of the LHG, water-level altitudes as much as $150 \mathrm{~m}$ shallower, and an east-west trend of the contours in southern Crater Flat. Other major differences occur south of Yucca Mountain, because Nye County EWDP data were not available to Czarnecki et al. (1997 [DIRS 141643]).

Larger-scale potentiometric-surface maps (Ervin et al. 1994 [DIRS 100633], Plate 1; Tucci and Burkhardt 1995 [DIRS 101060], Figure 4; Lehman and Brown 1996 [DIRS 149173], Figure 16) cover only a small portion of the site-scale model area in the vicinity of Yucca Mountain. The potentiometric-surface map by Ervin et al. (1994 [DIRS 100633], Plate 1) has water-level contour intervals of $0.25 \mathrm{~m}$. That map does not attempt to contour the areas of the LHG or the moderate hydraulic gradient to the west of the Solitario Canyon fault, but the general shape of the potentiometric contours is similar to the map constructed for this revision (Figure 6-1). The potentiometric-surface map by Tucci and Burkhardt (1995 [DIRS 101060], Figure 4) has contour intervals that vary from $0.5 \mathrm{~m}$ to $20 \mathrm{~m}$. Comparing the same potentiometric contours $(800-\mathrm{m}$ and 730-m contours) on that map and the map constructed for this revision reveals a similarity in shape, although the gradient in the LHG area in the Tucci and Burkhardt (1995 [DIRS 101060]) map is larger that the gradient presented in this revision. Other similarities include the steep gradient along Solitario Canyon and trough in the potentiometric surface along Fortymile Wash. The map by Lehman and Brown (1996 [DIRS 149173], Figure 16) presents an alternate concept of groundwater flow at Yucca Mountain, in which faults and fracture zones act as very 
permeable conduits for flow. Although such features play an important role in the groundwater flow system at Yucca Mountain, the map is based on corrections to water levels that may not be valid due to the large open intervals in some of the wells (Graves et al. 1997 [DIRS 101046], Appendix A). The map that Lehman and Brown present in their report (1996 [DIRS 149173], Figure 16) is also somewhat spatially distorted, so that it cannot be directly compared to other potentiometric maps constructed for Yucca Mountain.

The regional potentiometric surface of the Death Valley region (D'Agnese et al. 1997 [DIRS 100131], Plate 1) is at a much smaller scale than the potentiometric-surface map in this revision. The contour interval used by D'Agnese et al. (1997 [DIRS 100131], Plate 1) is $100 \mathrm{~m}$, resulting in only a few contour lines intersecting the SZ site-scale flow model area. As with the larger-scale potentiometric-surface maps, the same water-level contours that occur in the work of D'Agnese et al. (1997 [DIRS 100131], Plate 1) and the potentiometric-surface map in this revision can be compared. This comparison reveals that the potentiometric contours on both maps are similar, although, because D'Agnese et al. (1997 [DIRS 100131]) assume that water levels in Wells USW G-2 and UE-25 WT \#6 represent regional levels, the gradient in the LHG area is larger than the gradient presented in this revision.

Two recent alternative potentiometric-surface maps have been created as part of the ongoing work at Yucca Mountain. An alternative potentiometric-surface map was published by USGS (2001 [DIRS 154625]) and is shown on Figure 6-3. This map was created with a different conceptual model: (1) several faults were explicitly loaded into the gridding software and offsets of water level across the faults was allowed even for the case of the hydraulic gradient parallel to the strike of the fault, and (2) perched water levels in the northern portion of the area were assumed to represent the water table. The data used by the USGS (2001 [DIRS 154625]) to create the map are similar to the data used in the current report, and in many cases, the two maps are similar (e.g., consider the $750 \mathrm{~m}$ contour line). In general, the water-level contours from the repository to the Amargosa Desert are quite similar because there are numerous measured water levels to control the contour locations. However, in Crater Flat, north of Yucca Mountain, and in Jackass Flats, the water-level contours of both maps are quite different in some cases because of the limited number of water-level measurement points in those areas. In both maps, the direction of groundwater flow from the repository will be to the southeast toward Fortymile Canyon and then south to the Amargosa Valley. The impact of differences to water level contours in the outer areas on the groundwater flow paths from the repository is expected to be relatively small for several reasons. First, the flow paths in the repository area will be controlled by the local hydraulic gradient and hydraulic properties, both of which are constrained by site data. Second, the hydraulic properties in the outside areas are poorly constrained, thus there is significant latitude in the calibration process to adjust hydraulic parameters to match observed water-level contours. Third, corroborating evidence such as the analysis of water chemistry data (BSC 2004 [DIRS 170037], Appendix A) indicates that the groundwater flow lines calculated by BSC (2004 [DIRS 170037], Figure 6-43) are consistent with flow paths determined from the water chemistry data (BSC 2004 [DIRS 170037], Figure A6-62). The corroboration of the two analyses does not indicate that the contours provided on Figure 6-1 are exactly correct, rather, it means that the uncertainties in the water levels are not large enough to invalidate any of the potentiometric-surface maps. 
A second alternative water-level map of the portion of the study area near the repository was created to serve as the lower boundary condition for the unsaturated zone (UZ) flow model (BSC 2004 [DIRS 169855]). This map is shown on Figure 6-4. Near the repository, the map in this report (Figure 6-1) and Figure 6-4 are quite similar, but to the southeast and northwest, the two maps differ. In the high-gradient region, the water levels on Figure 6-4 are about $60 \mathrm{~m}$ higher at Well UE-25 WT \#6 than they are on Figure 6-1. The Solitario Canyon fault is believed to act as a barrier to flow and the water-level contours on Figure 6-1 are drawn to reflect that expectation. On Figure 6-4, the role of the fault is not explicitly reflected in the contours and the gradient through the Solitario Canyon on Figure 6-4 is more gradual than on Figure 6-1. As noted by BSC (2004 [DIRS 169855], Section 6.4.2 and p. 6-45) the two-step process used to create Figure 6-4 may have produced deviations in water table elevation that are typically less than $5 \mathrm{~m}$ in the area of the repository. The two-step process used the irregularly spaced observed water-level data and the contours from Figure 6-1 of this report to create a coarse grid of regularly spaced $(182.88-\mathrm{m}$ by $182.88-\mathrm{m})$ data. This data set was then interpolated again onto a $60.96-\mathrm{m}$ by $60.96-\mathrm{m}$ grid. The second grid was then edited to ensure no values less than $730 \mathrm{~m}$. Therefore, the potentiometric-surface map on Figure 6-4 is expected to show deviations from that shown on Figure 6-1.

Another consideration when examining the difference between the maps on Figure 6-4 and Figure 6-1 is the intended use of the map. The bottom of the UZ (Figure 6-4) is used to create the bottom of the UZ model grid and the primary concern is the depth to the water table from the repository level to the base of the UZ model.

From the perspective of the groundwater flow system, the 730-m contour on Figure 6-4 indicates flow directions that vary locally more than $180^{\circ}$, from north, to east, to south. As was noted earlier, uncertainty in the water levels could be larger than $1 \mathrm{~m}$ in the flat gradient region, however, the water-level differences from well to well are typically only a few tenths of 1 meter. The detail in the $730-\mathrm{m}$ water-level contour on Figure 6-4 is inappropriate because of the uncertainty in the measurements. 


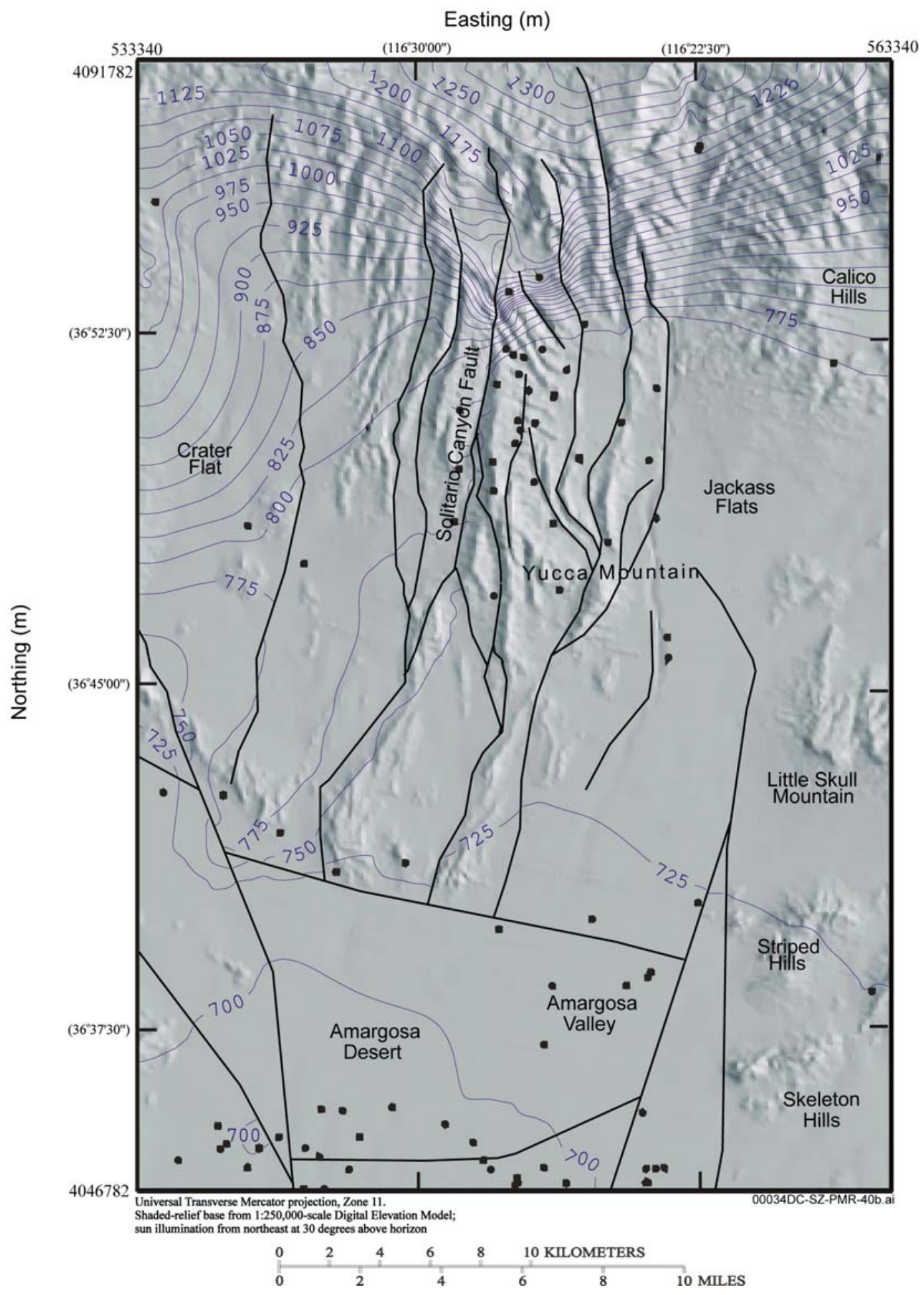

Source: USGS 2001 [DIRS 154625].

Figure 6-3. 2000 Potentiometric Surface used in the SZ Site-Scale Flow Model 


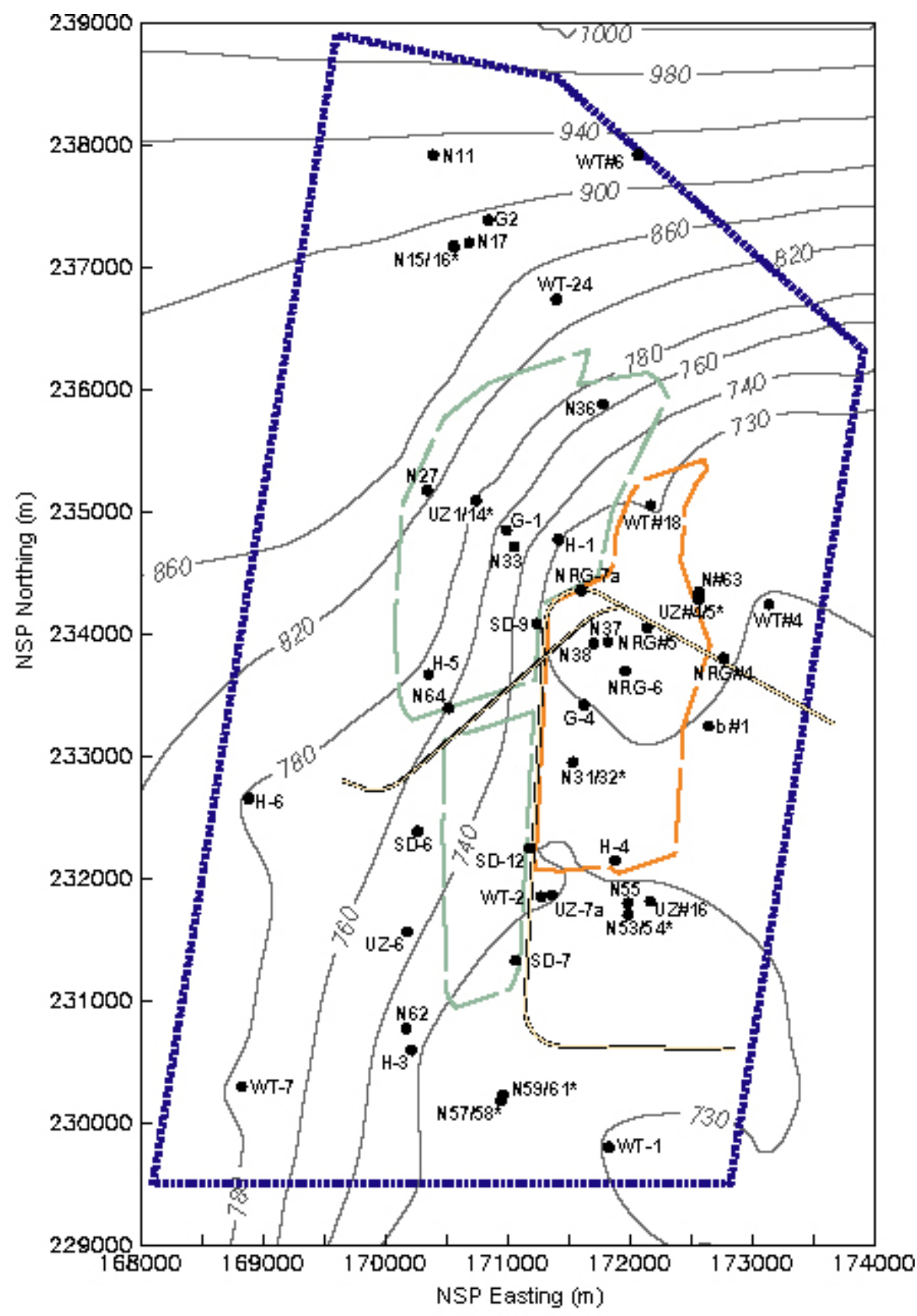

\begin{tabular}{|c|c|}
\hline \multicolumn{2}{|l|}{ LEGEND } \\
\hline "..."..."n uzzoce Mods Bandary & - 900 - Water Tatle Elevation Contars \\
\hline - zoce Repository Bandary & - Uz:n6 Borehdes \\
\hline $\begin{array}{l}\text { —— } 200 \text { Repository Lower Blodk } \\
\text { ESF and ECRB }\end{array}$ & $\begin{array}{l}\text { Note: * Denotes single location used for } \\
\text { agacerk borktoles. }\end{array}$ \\
\hline
\end{tabular}

Source: BSC 2004 [DIRS 169855], Figure 6-2.

Figure 6-4. Plan-View Schematic Showing Boreholes, Contoured Water Table (Elevations in Meters), UZ Model Boundary, Repository Outline, ESF, and ECRB Cross-Drift 


\section{INTENTIONALLY LEFT BLANK}




\section{CONCLUSIONS}

\subsection{SUMMARY}

An analysis of water levels measured in wells in and around the Yucca Mountain repository site was conducted to assess current water-level conditions, provide a potentiometric-surface map representative of the water table, and to provide target water levels for SZ site-scale flow model calibration. The analysis included an assessment of water-level data representative of the period of time in the early 1990s. The analysis included assessment of temporal trends, measurement errors, representativeness and vertical hydraulic head relationships. The water-level data are summarized into a series of tables that include the average water level to be used as calibration targets in SZ site-scale flow modeling. In addition, a potentiometric-surface map representative of conditions near the upper part of the SZ was created and presented on Figure 6-1.

\subsubsection{Water-Level Data}

Water-level altitudes in the SZ site-scale flow and transport model area range over $400 \mathrm{~m}$. The data distribution generally is very uneven, and the hydraulic character of the formations and the location of recharge areas, both of which influence the water level, are variable. As a result, water levels vary significantly over the SZ site-scale flow and transport model area.

Most of the water levels used in this analysis are composite levels in which water is produced from one or more hydrogeologic units or fracture zones as indicated in Appendix A, Table A-5, of this revision. Because of long open (uncased) or perforated/screened intervals, many boreholes intercept multiple permeable zones, resulting in a composite water-level altitude.

Potential errors in the potentiometric surface can result from the use of data from wells completed in potentially perched-water bodies, and from inaccuracies in the borehole site-location, land-surface altitude, water-level measurements, water-level altitude calculation, barometric fluctuations, water density corrections, and temporal fluctuations. Evaluation of methodology accuracy is documented in Appendix A, Tables A-6, A-7, and A-8. This information may be used to evaluate the representativeness of the water-level altitudes used in this analysis and to determine whether or not these altitudes represent the potentiometric surface of the upper SZ. Additional discussion of uncertainty in Section 6.5 points out that measurement uncertainty is a small part of the total uncertainty in the potentiometric-surface map.

In addition to measurement uncertainties, the range in water levels for a borehole can be used in the determination of an uncertainty of a mean water level at that site. Pumping is included in the flux rates used in the regional model (D’Agnese et al. 1997 [DIRS 100131], p. 48); therefore, water levels that may be influenced by pumping are included in the SZ site-scale flow model (BSC 2004 [DIRS 170037]). Because of the uncertainties in water levels discussed in the previous paragraph, the range in water-level altitudes and the possible causes of that variability should be taken into account during SZ site-scale flow model calibration. The calculated average water levels from Table A-1 are available from the TDMS under DTN: GS010908312332.002. 


\section{Vertical Head Differences}

Vertical head differences documented in Table 6-4 are variable throughout the SZ site-scale model area. The data are available in the TDMS under DTN: GS010908312332.003. Of the 17 sites at which vertical gradients have been evaluated, most gradients (12) are upward, and fewer (5) are downward. No correlation is evident in the spatial location of boreholes and the location of upward or downward vertical gradients. The reason for this lack of spatial correlation is probably because of the wide variation in the monitored hydrogeologic units and depths of the monitored intervals. Gradients appear to be upward from the Paleozoic carbonate aquifer and from the deepest parts of the volcanic-rock aquifers where potentiometric levels may be influenced by the underlying carbonates. A significant downward gradient exists at Borehole NC-EWDP-1DX at a paleospring deposit south of Crater Flat. A slight downward gradient at Borehole UE-25 J-13 may be indicative of recharge to the groundwater system along Fortymile Wash; however, the gradient is so small that it might only be an artifact of errors in water-level measurements.

\subsubsection{Potentiometric-Surface Map}

The 2000 potentiometric surface map presented in Figure 6-3 (USGS 2001 [DIRS 154625]) was used in the development of the SZ site-scale flow model. The 2001 potentiometric surface shown on Figure 6-1 provides a contour-map representation of the potentiometric surface from water-level data that were developed for this report and that are available from the TDMS under DTN: GS010608312332.001. This 2001 potentiometric surface presents an alternate concept of the LHG area north of Yucca Mountain to that presented in Revision 00 of this report (USGS 2001 [DIRS 154625]). In this revision, water levels in Boreholes USW G-2 and UE-25 WT \#6 are assumed to represent perched conditions and not regional potentiometric levels. If perched conditions do not actually exist at those boreholes and the water level in Well USW WT-24 also represents the regional potentiometric level, then the hydraulic gradient north of Yucca Mountain would be much greater than previously reported.

The 2001 potentiometric surface developed from the input data sets listed in Table 4-1 incorporates the potential errors and uncertainties identified in this revision. Hence, the accuracy of the potentiometric surface will vary spatially. In the repository area, the potentiometric surface may be characterized within one meter; however, in other areas within the SZ site-scale flow and transport model area, the uncertainty in water levels is much greater because of lack of data. Other sources of uncertainty include: (1) areas of perched-water, (2) water-level drawdown associated with pumping in the Amargosa Valley, and (3) the unknown effect of faults on water-level altitudes. The potentiometric surface presented herein does not strictly represent the water table, which is a concept reserved for the actual interface between the SZ and UZ. However, the potentiometric surface presented is a close and reasonable representation of the water table for the early 1990s (see Assumption 1 in Section 5.1).

Large portions of the SZ site-scale flow and transport model area contain no water-level data, and potentiometric contours drawn through those areas (indicated by dashed contours on Figure 6-1) are speculative and subject to other equally valid interpretations. Recent wells drilled as part of the Nye County EWDP near Fortymile Wash, south of Well UE-25 J-12, suggest that if the new data were incorporated into the potentiometric-surface map, the contours 
in that region may shift laterally about $1.5 \mathrm{~km}$. The resulting map (Figure 6-2) depicts a change in horizontal hydraulic gradient of 30 to 50 percent, well within the range of uncertainty in specific discharge. Potential errors in the location of those contours will be represented in the SZ site-scale model in one of three ways: (1) as potential errors in the saturated thickness of the uppermost saturated unit, (2) by a difference in which geologic unit constitutes the uppermost saturated unit, or (3) variation in the specific discharge along the flow path. The potentiometric surface uncertainty varies across the study area and can be grouped into two regions; one near the repository and downgradient, and the other near the boundaries of the model area.

The errors in the potentiometric surface will be larger in areas of less data (which are located near the western, northern, and eastern boundaries). The uncertainty in the potentiometric surface will lead to uncertainty in saturated thickness and uppermost saturated geologic units at the boundary of the model area. These uncertainties can be characterized by changes in the transmissivity of the model layers. Changes in transmissivity influence how much groundwater crosses the boundary into the model area. This uncertainty in the potentiometric surface at the boundary of the model is addressed in the SZ site-scale flow model via an analysis of the sensitivity and uncertainty to uncertainty in the boundary fluxes. The uncertainty in the potentiometric surface near the model boundaries will also impact the magnitude and direction of the specific discharge. This uncertainty in specific discharge in areas away from the repository is unlikely to cause significant uncertainty in the transport from the repository because the relatively high density of data near the repository provide sufficient data to constrain the flow system.

In the vicinity of the repository and downgradient toward Amargosa Desert, the uncertainty in the water-level elevation are going to be on the order of a few meters at most. This small error will not be important to either the saturated thickness or the identification of the uppermost saturated unit. The largest impact will be associated with the magnitude and direction of the specific discharge. In this case, the specific discharge is strongly influenced by the data in the vicinity of the repository. As noted in this document, the uncertainty in the potentiometric surface may change the magnitude of the hydraulic gradient by up to 50 percent and may alter the direction of the hydraulic gradient over portions of the area. To accommodate this uncertainty, the SZ site-scale flow model will need to account for different possible flow paths from the repository to the accessible environment.

The analysis of water levels presented in this report is adequate to define the shape of the potentiometric surface, the water levels appropriate for SZ site-scale flow model calibration, and the uncertainty associated with those water levels. The water levels are representative of the conditions near the top of the SZ for the period of the early to mid 1990s, the same time frame as the regional scale model (D'Agnese 1997 [DIRS 100131]). The uncertainty in the potentiometric-surface analysis can be incorporated into the SZ site-scale flow model and the resulting site-scale flow and transport abstraction model.

\subsection{INCORPORATION OF THE YMRP ACCEPTANCE CRITERIA}

A list of the YMRP criteria that have been addressed in this report were presented in Section 4.2. 


\subsection{Flow Paths in the Saturated Zone}

AC 1 (2) Adequacy of Descriptions of Flow Path Influences

The detailed description of the potentiometric-surface map in Section 6.4 includes geologic information that shows how potentiometric-surface contours may be affected in the SZ. Hydraulic gradient (which along with anisotropy define the flow paths) are determined from the potentiometric-surface contours.

AC1 (4) The Propagation of Initial and Boundary Conditions

The potentiometric surface is used as the boundary condition for the SZ site-scale flow model. It defines the upper surface of the computational mesh and as the hydraulic head conditions on the boundary of the model. Section 6.4 presents the potentiometric surface and data points that are propagated to the other models. Section 6.5 presents the consistency of the map on Figure 6-1 with other site-scale and regional scale potentiometric data.

\section{AC 1 (5) Sufficiency of Bases for Including FEPs}

The analyses in this report provide support to the technical bases for the FEPs that are presented in other documents. Section 6.2 and Table 6-1 show that this work supports the technical bases of several FEPs.

\section{AC 1(6) Adequacy of Flow Path Delineation}

The discussion in Section 6.4 of the relation between the distribution of water-level data and the complex geology in the SZ site-scale flow and transport model area and the potentiometric-surface map shows how natural site conditions have been considered to adequately delineate the flow paths in the SZ.

AC 1(10) Acceptable Approaches for Peer Review and Qualification

This report was developed in accordance with the QARD (DOE 2004 [DIRS 171539]), which commits to NUREGs 1297 and 1298. Moreover, compliance with the DOE procedures which are designed to implement these commitments is verified by audits by QA and other oversight activities. Accordingly, the guidance in NUREGs 1297 and 1298 has been followed, as appropriate.

\section{AC 2 (1) Justification of Values and Adequacy of Descriptions}

The description in Section 6.3 of how water-level data were collected shows that the values used to determine the potentiometric surface are adequately justified. The discussion in Section 6.4 on how the potentiometric-surface map was created and determined to be an accurate interpretation based on the available water-level data shows that the use, interpretation, and synthesis of the data was appropriate. 


\section{AC 2 (2) Data Sufficiency}

The variety and quality of data sources described in Section 6.3 show that sufficient data were collected to determine the potentiometric surface. The water-level data for the SZ site-scale flow and transport model were compiled from project data sources and NWIS water-level data.

\section{AC 2 (3) Appropriateness of Data Techniques}

The discussion in Section 6.1 on (1) how water-level data were collected by compiling existing USGS data and measuring water levels in boreholes throughout and adjacent to the SZ site-scale flow and transport model domain; (2) the use of professional judgment guided by specific criteria to determine whether water-level altitudes represented perched-water conditions; and (3) the assessment of long-term hydrograph records on the fluctuation of water level over time shows that data are based on appropriate techniques. 


\section{INTENTIONALLY LEFT BLANK}




\section{INPUTS AND REFERENCES}

The following is a list of the references cited in this document. Column 2 represents the unique six digit numerical identifier (the Document Input Reference System number), which is placed in the text following the reference callout (e.g., BSC 2001 [DIRS 155974]). The purpose of these numbers is to assist in locating a specific reference. Within the reference list, multiple sources by the same author (e.g., BSC 2001) are sorted alphabetically by title.

\subsection{DOCUMENTS CITED}

Blankennagel, R.K. 1967. Hydraulic Testing Techniques of Deep Drill Holes at 103092 Pahute Mesa, Nevada Test Site. Interagency Report: Special Studies I-1. Washington, D.C.: U.S. Geological Survey. ACC: NNA.19870729.0095.

BSC (Bechtel SAIC Company) 2001. Calibration of the Site-Scale Saturated 155974 Zone Flow Model. MDL-NBS-HS-000011 REV 00 ICN 01. Las Vegas, Nevada: Bechtel SAIC Company. ACC: MOL.20010713.0049.

BSC 2004. Development of Numerical Grids for UZ Flow and Transport 169855 Modeling. ANL-NBS-HS-000015 REV 02. Las Vegas, Nevada: Bechtel SAIC Company. ACC: DOC.20040901.0001.

BSC 2004. Features, Events, and Processes in SZ Flow and Transport. 170013 ANL-NBS-MD-000002, Rev. 03. Las Vegas, Nevada: Bechtel SAIC Company.

BSC 2004. Q-List. 000-30R-MGR0-00500-000-000 REV 00. Las Vegas, 168361 Nevada: Bechtel SAIC Company. ACC: ENG.20040721.0007.

BSC 2004. Saturated Zone Flow and Transport Model Abstraction. 170042 MDL-NBS-HS-000021, Rev. 02. Las Vegas, Nevada: Bechtel SAIC Company.

BSC 2004. Saturated Zone Site-Scale Flow Model. MDL-NBS-HS-000011, Rev. 02. Las Vegas, Nevada: Bechtel SAIC Company.

BSC 2004. Site-Scale Saturated Zone Transport. MDL-NBS-HS-000010, 170036 Rev. 02. Las Vegas, Nevada: Bechtel SAIC Company.

BSC 2004. Technical Work Plan for: Natural System - Saturated Zone Analysis 171421 and Model Report Integration. TWP-NBS-MD-000002 REV 02 ICN 01. Las Vegas, Nevada: Bechtel SAIC Company. ACC: DOC.20040818.0004.

Canori, G.F. and Leitner, M.M. 2003. Project Requirements Document. 166275 TER-MGR-MD-000001 REV 02. Las Vegas, Nevada: Bechtel SAIC Company. ACC: DOC.20031222.0006. 
Craig, R.W. and Robison, J.H. 1984. Geohydrology of Rocks Penetrated by Test Investigations Report 84-4248. Denver, Colorado: U.S. Geological Survey. ACC: NNA.19890905.0209.

CRWMS M\&O (Civilian Radioactive Waste Management: System Management 100353 and Operating Contractor) 1998. Saturated Zone Flow and Transport Expert Elicitation Project. Deliverable SL5X4AM3. Las Vegas, Nevada: CRWMS M\&O. ACC: MOL.19980825.0008.

Czarnecki, J.B. and Waddell, R.K. 1984. Finite-Element Simulation of

101042

Ground-Water Flow in the Vicinity of Yucca Mountain, Nevada-California.

Water-Resources Investigations Report 84-4349. Denver,

Colorado: U.S. Geological Survey. ACC: NNA.19870407.0173.

Czarnecki, J.B.; Faunt, C.C.; Gable, C.W.; and Zyvoloski, G.A. 1997.

Hydrogeology and Preliminary Three-Dimensional Finite-Element Ground-Water Flow Model of the Site Saturated Zone, Yucca Mountain, Nevada. Milestone SP23NM3. Denver, Colorado: U.S. Geological Survey.

ACC: MOL.19990812.0180.

D'Agnese, F.A.; Faunt, C.C.; Turner, A.K.; and Hill, M.C. 1997. Hydrogeologic

Evaluation and Numerical Simulation of the Death Valley Regional

Ground-Water Flow System, Nevada and California. Water-Resources

Investigations Report 96-4300. Denver, Colorado: U.S. Geological Survey.

ACC: MOL.19980306.0253.

DOE (U.S. Department of Energy) 2004. Quality Assurance Requirements and Description. DOE/RW-0333P, Rev. 16. Washington, D.C.: U.S. Department of Energy, Office of Civilian Radioactive Waste Management.

ACC: DOC.20040823.0004.

Ervin, E.M.; Luckey, R.R.; and Burkhardt, D.J. 1994. Revised

Potentiometric-Surface Map, Yucca Mountain and Vicinity, Nevada.

Water-Resources Investigations Report 93-4000. Denver, Colorado:

U.S. Geological Survey. ACC: NNA.19930212.0018.

Fenelon, J.M. 2000. Quality Assurance and Analysis of Water Levels in Wells on 160881 Pahute Mesa and Vicinity, Nevada Test Site, Nye County, Nevada.

Water-Resources Investigations Report 00-4014. Carson City, Nevada:

U.S. Geological Survey. ACC: MOL.20030904.0304.

Fenelon, J.M. and Moreo, M.T. 2002. Trend Analysis of Ground-Water Levels and Spring Discharge in the Yucca Mountain Region, Nevada and California, 1960-2000. Water-Resources Investigations Report 02-4178. Carson City, Nevada: U.S. Geological Survey. ACC: MOL.20030812.0306. 
Freeze, R.A. and Cherry, J.A. 1979. Groundwater. Englewood Cliffs,

Fridrich, C.J.; Dudley, W.W., Jr.; and Stuckless, J.S. 1994. "Hydrogeologic

100575

Analysis of the Saturated-Zone Ground-Water System, Under Yucca Mountain, Nevada." Journal of Hydrology, 154, 133-168. Amsterdam, The Netherlands:

Elsevier. TIC: 224606.

Graves, R.P. 1998. Water Levels in the Yucca Mountain Area, Nevada, 1996.

155411

Open-File Report 98-169. Denver, Colorado: U.S. Geological Survey.

ACC: MOL.19981117.0340.

Graves, R.P. 2001. "Re: WT-24." E-mail from R.P. Graves (USGS) to P. Tucci

155942 (USGS), August 29, 2001. ACC: MOL.20010907.0002.

Graves, R.P.; Tucci, P.; and O’Brien, G.M. 1997. Analysis of Water-Level Data in the Yucca Mountain Area, Nevada, 1985-95. Water-Resources Investigations Report 96-4256. Denver, Colorado: U.S. Geological Survey. ACC: MOL.19980219.0851.

Lehman, L.L. and Brown, T.P. 1996. Summary of State of Nevada - Funded 149173 Studies of the Saturated Zone at Yucca Mountain, Nevada, Performed by L. Lehman \& Associates, Inc. Burnsville, Minnesota: L. Lehman and Associates. TIC: 231894

Luckey, R.R.; Tucci, P.; Faunt, C.C.; Ervin, E.M.; Steinkampf, W.C.; D’Agnese, 100465 F.A.; and Patterson, G.L. 1996. Status of Understanding of the Saturated-Zone Ground-Water Flow System at Yucca Mountain, Nevada, as of 1995.

Water-Resources Investigations Report 96-4077. Denver, Colorado: U.S. Geological Survey. ACC: MOL.19970513.0209.

NRC (U.S. Nuclear Regulatory Commission) 2003. Yucca Mountain Review 163274 Plan, Final Report. NUREG-1804, Rev. 2. Washington, D.C.: U.S. Nuclear Regulatory Commission, Office of Nuclear Material Safety and Safeguards. TIC: 254568.

O’Brien, G. 1998. "Milestone SPH40NM4 (Level 4) - Memo to TPO: Possible Perched-Water Occurrences North of Yucca Mountain." Memorandum from G. O'Brien (USGS) to R. Craig (USGS), September 28, 1998, with attachment, "Perched-Water Analysis Death Valley Region." ACC: MOL.19981130.0221; MOL.19990113.0213.

Paces, J.B.; Ludwig, K.R.; Peterman, Z.E.; and Neymark, L.A. 2002. ${ }^{، 234} \mathrm{U} /{ }^{238} \mathrm{U}$ 158817 Evidence for Local Recharge and Patterns of Ground-Water Flow in the Vicinity of Yucca Mountain, Nevada, USA.” Applied Geochemistry, 17, ([6]), 751-779. New York, New York: Elsevier. TIC: 252809. 
Rousseau, J.P.; Kwicklis, E.M.; and Gillies, D.C., eds. 1999. Hydrogeology of the 102097 Unsaturated Zone, North Ramp Area of the Exploratory Studies Facility, Yucca Mountain, Nevada. Water-Resources Investigations Report 98-4050. Denver, Colorado: U.S. Geological Survey. ACC: MOL.19990419.0335.

Savard, C.S. 2001. Water Levels in the Yucca Mountain Area, Nevada, 1999. 165604 Open-File Report 01-343. Denver, Colorado: U.S. Geological Survey. ACC: MOL.20020513.0059.

Spengler, R. 2001. "Pz in NC-EWDP-2DB." E-mail from R. Spengler to 155823 P. McKinley, August 30, 2001. ACC: MOL.20010907.0001.

Thordarson, W. 1983. Geohydrologic Data and Test Results from Well J-13, 101057 Nevada Test Site, Nye County, Nevada. Water-Resources Investigations Report 83-4171. Denver, Colorado: U.S. Geological Survey.

ACC: NNA.19870518.0071.

Tucci, P. 2001. Segment of SN-USGS-SCI-126-V1: Revision of Water Level 155410 AMR (ANL-NBS-HS-000034, Rev 00/ICN 01). Scientific Notebook SN-USGS-SCI-126-V1. ACC: MOL.20010712.0271.

Tucci, P. and Burkhardt, D.J. 1995. Potentiometric-Surface Map, 1993, Yucca 101060 Mountain and Vicinity, Nevada. Water-Resources Investigations Report 95-4149. Denver, Colorado: U.S. Geological Survey. ACC: MOL.19960924.0517.

USGS (U.S. Geological Survey) n.d. Raw Data Field Notebook \#1, Ue25P\#1 Book I of II Record. Denver, Colorado: U.S. Geological Survey. ACC: MOL.20000301.0894.

USGS 2001. Water-Level Data Analysis for the Saturated Zone Site-Scale Flow and Transport Model. ANL-NBS-HS-000034 REV 00 ICN 01. Denver, Colorado: U.S. Geological Survey. ACC: MOL.20010405.0211.

USGS 2004. Water-Level Data Analysis for the Saturated Zone Site-Scale Flow and Transport Model. ANL-NBS-HS-000034 REV 01, with errata. Denver, Colorado: U.S. Geological Survey. ACC: MOL.20020209.0058; MOL.20020917.0136; DOC.20040303.0006.

Weast, R.C., ed. 1985. CRC Handbook of Chemistry and Physics. 66th Edition. 111561 Boca Raton, Florida: CRC Press. TIC: 216054.

Weir, J.E., Jr., and Nelson, J.W. 1976. Operation and maintenance of a deep-well 170983 water-level measuring device, the "Iron Horse". Water-Resources Investigations 76-27. 28 p. Denver, CO: U.S. Geological Survey. 
Williams, N.H. 2003 “Contract No. DE-AC28-01RW12101 - Transmittal of

Report Technical Basis Document No. 11: Saturated Zone Flow and Transport Revision 2 Addressing Twenty-Five Key Technical Issue (KTI) Agreements Related to Saturated Zone Flow and Transport." Letter from N.H. Williams (BSC) to C.M. Newbury (DOE/ORD), September 30, 2003, MP: cg - 0930038958, with enclosure. ACC: MOL.20040105.0270.

\subsection{STANDARDS, REGULATIONS, AND PROCEDURES}

10 CFR 63. ENERGY: Disposal of High-Level Radioactive Wastes in a Geologic Repository at Yucca Mountain, Nevada. Readily Available.

AP-2.22Q, Rev. 1, ICN 1. Classification Analyses and Maintenance of the Q-List. Washington, D.C.: U.S. Department of Energy, Office of Civilian Radioactive Waste Management. ACC: DOC.20040714.0002.

AP-2.27Q, Rev. 1, ICN 4. Planning for Science Activities. Washington, D.C.: U.S. Department of Energy, Office of Civilian Radioactive Waste Management. ACC: DOC.20040610.0006.

LP-SI.11Q-BSC, Rev. 0 ICN 1. Software Management. Washington, D.C.: U.S. Department of Energy, Office of Civilian Radioactive Waste Management. ACC: DOC.20041005.0008.

AP-SIII.2Q, Rev 1, ICN 2. Qualification of Unqualified Data. Washington, D.C.: U.S. Department of Energy, Office of Civilian Radioactive Waste Management. ACC: DOC.20040127.0008.

AP-SIII.9Q, Rev 1, ICN 7. Scientific Analyses. Washington, D.C.: U.S. Department of Energy, Office of Civilian Radioactive Waste Management. ACC: DOC.20040920.0001.

\subsection{SOURCE DATA, LISTED BY DATA TRACKING NUMBER}

GS000400002332.001. Digital Elevation Models Death Valley East Scale 148924 1:250,000. Submittal date: 04/12/2000.

GS000408312312.001. Water-Level Measurements at UE-25 C \#2 and C \#3, 1989. Submittal date: $04 / 10 / 2000$.

GS000608312312.003. Water-Level Altitude Data from the Periodic Network, January 1999 through March 1999. Submittal date: 07/10/2000.

GS000608312312.004. Revised Water-Level Altitude Data from the Periodic Network, First Quarter 1995. Submittal date: 06/27/2000. 
GS000708312312.005. Water-Level Altitude Data, 1993. Submittal date:

$07 / 10 / 2000$.

GS000808312312.007. Ground-Water Altitudes from Manual Depth-to-Water

155270

Measurements at Various Boreholes November 1998 through December 1999.

Submittal date: 08/21/2000.

GS001208312312.009. Ground-Water Altitudes from Manual Depth-to-Water

Measurements at Various Boreholes January through June 2000. Submittal date: $12 / 29 / 2000$.

GS010808312312.003. Ground-Water Altitudes from Manual Depth-to-Water

168536

Measurements at Various Boreholes, January through June 2001. Submittal date: $08 / 28 / 2001$.

GS010908314221.001. Geologic Map of the Yucca Mountain Region, Nye

County, Nevada. Submittal date: 01/23/2002.

GS920408312314.009. Geohydrology of Rocks Penetrated by Test Well

148168 UE-25p\#1 (UE-25 p\#1), Yucca Mountain Area, Nye County, Nevada. Submittal date: $04 / 27 / 1992$.

GS930408312132.007. Geohydrologic Data and Test Results from Well J-13, Nevada Test Site, Nye County, Nevada. Submittal date: 04/23/1993.

129625

GS930408312312.015. Water Levels in the Yucca Mountain Area, Nevada, 1990-

148665

91. Submittal date: 04/28/1993.

GS931008312312.025. Water Levels in Periodically Measured Wells in the

148668

Yucca Mountain Area, Nevada, 1981-87. Submittal date: 04/23/1993.

GS950108312312.001. Water-Level Altitude Data from the Periodic Network, Fourth Quarter 1994. Submittal date: 01/19/1995.

GS950508312312.005. Potentiometric-Surface Map, 1993, Yucca Mountain and 105068 Vicinity, Nevada. Submittal date: 06/06/1995.

GS950808312312.008. Water-Level Altitude Data from USW H-1, 12/20/1994.

171370

Submittal date: 08/30/1995.

GS960208312312.003. 28 Water-Level Measurements from the Periodic

148672

Network, Third Quarter, 1995 (7/1/95 - 9/30/95). Submittal date: 02/20/1996.

GS960908312312.010. Analysis of Water-Level Data in the Yucca Mountain

105063 Area, Nevada, 1985-1995. Submittal date: 09/19/1996. 
GS970600012847.001. Water-Level Altitude Data at GEXA Well 4 and at USW

G-4. Submittal date: 06/11/1997.

GS980308312312.004. Water Levels in the Yucca Mountain Area, Nevada, 1996.

155272

Submittal date: 03/31/1998.

GS990908312312.005. Water-Level Altitude Data, April - June 1999. Submittal

155269 date: $09 / 20 / 1999$.

GS991100002330.001. Water Level Data for Yucca Mountain Region and

122818

Amargosa Desert. Submittal date: 03/29/2000.

GS991208314221.001. Geologic Map of the Yucca Mountain Region. Submittal

145263 date: $12 / 01 / 1999$.

MO0008WTRALTG4.000. Water-Table-Altitude Data for Well USW G-4, 155456 Yucca Mountain Area, Nye County, Nevada. Submittal date: 08/24/2000.

MO0011ELLOCAMD.000. Locations and Elevations for Selected Wells in the 153274 Yucca Mountain Region and Amargosa Desert from the USGS NWIS Database. Submittal date: 11/06/2000.

MO0103COV01031.000. Coverage: BORES3Q. Submittal date: 03/22/2001.

155271

MO0107COV01057.000. Coverage: NCEWDPS. Submittal date: 07/18/2001.

157194

MO0111DQRWLNYE.002. Water Level Data from Westbay Instrumented

157172

Borehole NC-EWDP-1S. Submittal date: 11/29/2001.

MO0111DQRWLNYE.003. Water Level Data from Westbay Instrumented

157173

Borehole NC-EWDP-3S. Submittal date: 11/29/2001.

MO0111DQRWLNYE.004. Water Level Data from Westbay Instrumented

157174

Borehole NC-EWDP-9SX. Submittal date: 11/29/2001.

MO0112DQRWLNYE.005. Well Completion Diagram for Borehole

157175

NC-EWDP-4PA. Submittal date: 12/03/2001.

MO0112DQRWLNYE.006. Well Completion Diagram for Borehole

157176

NC-EWDP-4PB. Submittal date: 12/04/2001.

MO0112DQRWLNYE.007. Well Completion Diagram for Borehole

157177

NC-EWDP-7S. Submittal date: 12/04/2001.

MO0112DQRWLNYE.008. Well Completion Diagram for Borehole

157178

NC-EWDP-5SB. Submittal date: 12/04/2001. 
MO0112DQRWLNYE.009. Well Completion Diagram for Borehole

NC-EWDP-15P. Submittal date: 12/04/2001.

MO0112DQRWLNYE.010. Well Completion Diagram for Borehole

157180

NC-EWDP-12PA. Submittal date: 12/04/2001.

MO0112DQRWLNYE.011. Well Completion Diagram for Borehole

157181

NC-EWDP-9SX. Submittal date: 12/04/2001.

MO0112DQRWLNYE.012. Well Completion Diagram for Borehole

157182

NC-EWDP-12PB. Submittal date: 12/04/2001.

MO0112DQRWLNYE.013. Well Completion Diagram for Borehole

157183

NC-EWDP-12PC. Submittal date: 12/04/2001.

MO0112DQRWLNYE.014. Well Completion Diagram for Borehole

157184

NC-EWDP-19P. Submittal date: 12/04/2001.

MO0112DQRWLNYE.015. Well Completion Diagram for Borehole

157200

NC-EWDP-3S. Submittal date: 12/04/2001.

MO0112DQRWLNYE.016. Well Completion Diagram for Borehole

157185

NC-EWDP-3D. Submittal date: 12/04/2001.

MO0112DQRWLNYE.017. Well Completion Diagram for Borehole

157186

NC-EWDP-1DX. Submittal date: 12/05/2001.

MO0112DQRWLNYE.018. Well Completion Diagram for Borehole

157187

NC-EWDP-19D. Submittal date: 12/05/2001.

MO0112DQRWLNYE.019. Multi-Level Monitoring Port Depths in Nye County

157188

Boreholes NC-EWDP-1S, -3S and -9SX. Submittal date: 12/05/2001.

MO0112DQRWLNYE.020. Water Level Depth Data for Nye County Boreholes

157199

NC-EWDP-2D and -2DB. Submittal date: 12/05/2001.

MO0112DQRWLNYE.021. Multilevel Piezometer Casing Log for Borehole

157189

NC-EWDP-9SX. Submittal date: 12/05/2001.

MO0112DQRWLNYE.022. Multilevel Piezometer Casing Log for Borehole

157190

NC-EWDP-3S. Submittal date: 12/05/2001.

MO0112DQRWLNYE.023. Multilevel Piezometer Casing Log for Borehole

157191

NC-EWDP-1S. Submittal date: 12/05/2001.

MO0112DQRWLNYE.024. EWDP Phase I Manual Water Level Measurements.

157192 Submittal date: 12/06/2001. 
MO0112DQRWLNYE.025. EWDP Phase II Manual Water Level Measurements. 157193 Submittal date: 12/06/2001.

MO0203GSC02034.000. As-Built Survey of Nye County Early Warning Drilling 168375 Program (EWDP) Phase III Boreholes NC-EWDP-10S, NC-EWDP-18P, and NC-EWDP-22S - Partial Phase III List. Submittal date: 03/21/2002.

MO0206GSC02074.000. As-Built Survey of Nye County Early Warning Drilling Program (EWDP) Phase III Boreholes, Second Set. Submittal date: 06/03/2002.

MO0307GSC03094.000. As-Built Survey of Nye County Early Warning Drilling 170556 Program Phase IV Boreholes EWDP-16P, EWDP-27P \& EWDP-28P. Submittal date: $07 / 14 / 2003$.

MO0401COV03168.000. Coverage: NCEWDPS. Submittal date: 01/27/2004. 168534 MO0405NYE05819.215. Manual Water Level Data for EWDP Phase I Wells 170539 from 05/02 - 08/03, Phase II Wells from 05/02 - 08/03, Phase III Wells from 11/02 - 8/03 and Phase IV Wells from 01/03 - 08/03. Submittal date: 05/20/2004.

MO0407SEPFEPLA.000. LA FEP List. Submittal date: 07/20/2004.

170760

TM0000SD7SUPER.001. USW SD-7 Structure Logs (48.5' to 2675.1'). 168540 Submittal date: 12/14/1995.

TM0000SD9SUPER.002. USW SD-9 Structure Logs (53.6' to 2223.1'). Submittal date: 12/14/1995.

TM000SD12SUPER.003. USW SD-12 Structure Logs (5.3' - 2166.3). Submittal 168543 date: $05 / 22 / 1996$.

\subsection{OUTPUT DATA, LISTED BY DATA TRACKING NUMBER}

GS010608312332.001. Potentiometric-Surface Map, assuming perched conditions north of Yucca Mountain, in the Saturated Zone Site-Scale Flow and Transport Model Area. Submittal date: 6/18/2001.

GS010908312332.002. Borehole Data from Water-Level Data Analysis for the Saturated Zone Site-Scale Flow and Transport Model. Submittal date: $10 / 02 / 2001$.

GS010908312332.003. Vertical Head Differences from Water-Level Data Analysis for the Saturated Zone Site-Scale Flow and Transport Model. Submittal date: $09 / 28 / 2001$ 
MO0409SEPPSMPC.000. Potentiometric-Surface Map Showing Possible Changes After Including EWDP Phases III and IV Wells. Submittal date: $09 / 29 / 2001$.

\subsection{SOFTWARE CODES}

USGS (U.S. Geological Survey) 2000. Software Code: ARCINFO. V7.2.1.

148304 10033-7.2.1-01.

Dynamic Graphics 2000. Software Code: EARTHVISION. V5.1. SGI/IRIX 6.5.

167994 10174-5.1-00. 
APPENDIX A

BOREHOLE DATA 
The following sections describe the information pertaining to terms used in Appendix A.

\section{USGS SITE IDENTIFICATION}

Unique site IDs are assigned to each borehole for which the USGS maintains water-level data. Boreholes that contain multiple monitoring zones are assigned a unique site ID for each of the different zones. The site IDs are different from the site ID for the entire borehole, but usually contain a portion of the borehole site ID. Where more than one site ID for a given borehole exists (multiple monitoring zones), the site ID for the entire borehole is used in Appendix A.

\section{Site Name}

Except for private wells, the common borehole site name available for a given site was recorded. The private wells have been given identifier $\mathrm{PW}-\mathrm{n}$, where $\mathrm{n}$ is an integer. The private wells identified in the appendices by PW are also identified the same way on the well location map (Figure 1-2). For comparison to the data sources where private well names are used, a list with the well name and the private well identifier is given in Table A-9. 


\section{TABLE A-1: EASTING, NORTHING, LAND SURFACE ALTITUDE, MEAN WATER-LEVEL ALTITUDE (DTN: GS010908312332.002)}

\section{Easting and Northing}

Coordinates for boreholes used in this table are from DTNs: MO0011ELLOCAMD.000 [DIRS 153274] (see Assumption 5.3), MO0103COV01031.000 [DIRS 155271], and MO0401COV03168.000 [DIRS 168534]. DTN: MO0107COV01057.000 [DIRS 157194] (which is superceded by DTN: MO0401COV03168.000) was used for coordinates as presented in Figures 6-1 and 6-2 and is justified for intended use in this document in Section 4.1. The Impact Review Action Notice (IRAN) 3505 (MOL.20040304.0206) indicates no impact to the figures in this document as a result of superceding the previous DTN. UTM easting and northing were calculated using EarthVision Version 5.1. The latitude/longitude, or state plane coordinate, was projected into UTM (meters, Zone 11, North American Datum 1927) coordinates and rounded to the nearest meter.

\section{Land-Surface Altitude Above Sea Level (meters)}

The land-surface altitudes for boreholes used in this analysis are from DTNs: MO0011ELLOCAMD.000 (see Assumption 5.3), MO0107COV01057.000, and MO0103COV01031.000. The altitude was converted from feet to meters by the following formula, where necessary:

$$
\begin{aligned}
& \text { Altitude }(\mathrm{ft}) \times 0.3048(\mathrm{~m} / \mathrm{ft})=\text { Altitude (meters) } \\
& \text { For example, } 2401.52 \mathrm{ft} \times 0.3048 \mathrm{~m} / \mathrm{ft}=731.98 \mathrm{~m}
\end{aligned}
$$

The altitude was subsequently rounded to the nearest tenth of 1 meter for use in this table.

\section{Mean Water-Level Altitude Above Sea Level (Meters)}

Mean water-level altitudes were calculated as time averages over the period of available measurements. For the wells tabulated by Graves et al. (1997 [DIRS 101046], Table 2) and DTN: GS960908312312.010 [DIRS 105063], monthly mean water-level altitudes computed from hourly transducer data and periodic manual water-level altitude measurements were used to compute the mean water-level altitude. The mean water level for each site was not included in the work of Graves et al. (1997 [DIRS 10146]; DTN: GS960908312312.010), but was calculated based on the available record. An example calculation is:

$$
(730.98+731.07+731.09) / 3=731.05
$$

The altitude was subsequently rounded to the nearest tenth of 1 meter for use in this table.

In addition, the following exceptions were made when calculating the mean:

- NDOT well: Deleted the 1972 measurement $(2,291.8 \mathrm{ft})$ from the calculation of the average. It is anomalously low and not representative of average conditions in the borehole. 
- PW-16 well: Deleted the 1961 measurement $(2,342.0 \mathrm{ft})$ from the calculation of the average. It is anomalously high and not representative of average conditions in the borehole.

- PW-20 well: Deleted the 1958 measurement (2,362.0 ft) from the calculation of the average. It is anomalously high and not representative of average conditions in the borehole.

- PW-30 well: Deleted the 1963 measurement $(2,324.6 \mathrm{ft})$ from the calculation of the average. It is anomalously low and not representative of average conditions in the borehole.

- PW-31 well: Deleted the 2 measurements of 2,333.8 ft (12/20/1961) and 2,335.3 ft $(04 / 09 / 1991)$ from the calculation of the average. They are anomalously low and not representative of average conditions in the borehole.

- PW-21 well: Used the data obtained after 1982 as being more representative of the calibration period.

- Airport well: Deleted the 1964 measurement $(2,348.8 \mathrm{ft})$ from the calculation of the average. It is anomalously high and not representative of average conditions in the borehole.

- GEXA well 4: Deleted the 1991 measurement (3133.2 ft.) from the calculations of the average. It is anomalously low and not representative of average conditions in the borehole.

- Nye County EWDP boreholes: Water-level measurements made prior to development of the well were not used to calculate the mean. Additionally, water-level measurements obtained by transducers that were believed to be unreliable (based on comparison to measurements in other zones within the borehole and/or comparison to other boreholes) were not used. Those individual unreliable measurements consist of thousands of data points and are too numerous to list here.

Table A-1. Easting, Northing, Land Surface Altitude, Mean Water-Level Altitude (DTN: GS010908312332.002)

\begin{tabular}{|l|l|l|l|l|l|}
\hline \multicolumn{1}{|c|}{ USGS Site ID } & \multicolumn{1}{|c|}{ Site Name } & $\begin{array}{c}\text { Easting } \\
\text { (UTM) }\end{array}$ & $\begin{array}{c}\text { Northing } \\
\text { (UTM) }\end{array}$ & $\begin{array}{c}\text { Land-surface } \\
\text { Altitude } \\
\text { (meters) }\end{array}$ & $\begin{array}{c}\text { Mean Water- } \\
\text { Level Altitude } \\
\text { (meters) }\end{array}$ \\
\hline 365629116222602 & UE-29 a\#2 & 555753 & 4088351 & 1215.4 & 1187.7 \\
\hline 365520116370301 & GEXA Well 4 & 534069 & 4086110 & 1198.1 & 1009.0 \\
\hline 365340116264601 & UE-25 WT\#6 & 549352 & 4083103 & 1314.8 & 1034.6 \\
\hline 365322116273501 & USW G-2 & 548143 & 4082542 & 1554.0 & 1020.2 \\
\hline 365239116253401 & UE-25 WT\#16 & 551146 & 4081234 & 1210.9 & 738.3 \\
\hline 365208116274001 & USW UZ-14 & 548032 & 4080261 & 1348.9 & 779.0 \\
\hline 365207116264201 & UE-25 WT\#18 & 549468 & 4080238 & 1336.4 & 730.8 \\
\hline
\end{tabular}


Table A-1. Easting, Northing, Land Surface Altitude, Mean Water-Level Altitude (DTN: GS010908312332.002) (Continued)

\begin{tabular}{|c|c|c|c|c|c|}
\hline USGS Site ID & Site Name & $\begin{array}{c}\text { Easting } \\
\text { (UTM) }\end{array}$ & $\begin{array}{l}\text { Northing } \\
\text { (UTM) }\end{array}$ & $\begin{array}{c}\text { Land-surface } \\
\text { Altitude } \\
\text { (meters) } \\
\end{array}$ & $\begin{array}{c}\text { Mean Water- } \\
\text { Level Altitude } \\
\text { (meters) }\end{array}$ \\
\hline 365200116272901 & USW G-1 & 548306 & 4080016 & 1325.9 & 754.2 \\
\hline 365147116185301 & UE-25 a\#3 & 561084 & 4079697 & 1385.6 & 748.3 \\
\hline 365140116260301 & UE-25 WT\#4 & 550439 & 4079412 & 1169.3 & 730.8 \\
\hline 365116116233801 & UE-25 WT\#15 & 554034 & 4078694 & 1083.2 & 729.2 \\
\hline 365114116270401 & USW G-4 & 548933 & 4078602 & 1269.5 & 730.6 \\
\hline 365105116262401 & UE-25 a\#1 & 549925 & 4078330 & 1199.2 & 731.0 \\
\hline 365032116243501 & UE-25 WT\#14 & 552630 & 4077330 & 1076.4 & 729.7 \\
\hline 365023116271801 & USW WT-2 & 548595 & 4077028 & 1301.4 & 730.6 \\
\hline 364947116254300 & UE-25 c\#1 & 550955 & 4075933 & 1130.6 & 730.2 \\
\hline 364947116254501 & UE-25 c\#3 & 550930 & 4075902 & 1132.4 & 730.2 \\
\hline 364947116254401 & UE-25 c\#2 & 550955 & 4075871 & 1132.2 & 730.2 \\
\hline 364945116235001 & UE-25 WT\#13 & 553730 & 4075827 & 1032.5 & 729.1 \\
\hline 364933116285701 & USW WT- 7 & 546151 & 4075474 & 1196.9 & 775.8 \\
\hline 364916116265601 & USW WT- 1 & 549152 & 4074967 & 1201.4 & 730.4 \\
\hline 364905116280101 & USW G-3 & 547543 & 4074619 & 1480.6 & 730.5 \\
\hline 364828116234001 & UE-25 J-13 & 554017 & 4073517 & 1011.3 & 728.4 \\
\hline 364825116290501 & USW WT-10 & 545964 & 4073378 & 1123.4 & 776.0 \\
\hline 364822116262601 & UE-25 WT\#17 & 549905 & 4073307 & 1124.0 & 729.7 \\
\hline 365821116343701 & USW VH-2 & 537738 & 4073214 & 974.5 & 810.4 \\
\hline 364757116245801 & UE-25 WT\#3 & 552090 & 4072550 & 1030.0 & 729.6 \\
\hline 364732116330701 & USW VH-1 & 539976 & 4071714 & 963.5 & 779.4 \\
\hline 364656116261601 & UE-25 WT\#12 & 550168 & 4070659 & 1074.7 & 729.5 \\
\hline 364649116280201 & USW WT-11 & 547542 & 4070428 & 1094.1 & 730.7 \\
\hline 364554116232400 & UE-25 J-12 & 554444 & 4068774 & 953.6 & 727.9 \\
\hline 364528116232201 & UE-25 JF\#3 & 554498 & 4067974 & 944.4 & 727.8 \\
\hline 364105116302601 & Cind-R-Lite Well & 544027 & 4059809 & 830.8 & 729.8 \\
\hline 363907116235701 & PW-1 & 553704 & 4056228 & 819.9 & 718.4 \\
\hline 363836116234001 & PW-2 & 553808 & 4055459 & 811.4 & 702.8 \\
\hline 363840116235000 & PW-3 & 553883 & 4055398 & 813.8 & 704.1 \\
\hline 363840116234001 & PW-4 & 554131 & 4055399 & 810.8 & 705.6 \\
\hline 363840116233501 & PW-5 & 554008 & 4055337 & 811.4 & 701.6 \\
\hline 363835116234001 & NDOT Well & 553685 & 4055242 & 809.8 & 705.4 \\
\hline 363742116263201 & PW-6 & 549863 & 4054911 & 795.5 & 706.7 \\
\hline 363830116241401 & Airport Well & 552818 & 4054929 & 804.3 & 705.3 \\
\hline 363815116175901 & TW- 5 & 562604 & 4054686 & 931.5 & 725.1 \\
\hline 363711116263701 & PW-7 & 549746 & 4053647 & 783.9 & 707.7 \\
\hline 363621116263201 & PW-8 & 549679 & 4052322 & 774.2 & 704.4 \\
\hline 363549116305001 & Nye County Development Co. & 543481 & 4050069 & 742.2 & 694.3 \\
\hline 363523116353701 & PW-9 & 536350 & 4050006 & 731.8 & 691.9 \\
\hline 363525116325601 & PW-10 & 540673 & 4049994 & 735.2 & 694.3 \\
\hline
\end{tabular}


Table A-1. Easting, Northing, Land Surface Altitude, Mean Water-Level Altitude (DTN: GS010908312332.002) (Continued)

\begin{tabular}{|c|c|c|c|c|c|}
\hline USGS Site ID & Site Name & $\begin{array}{c}\text { Easting } \\
\text { (UTM) }\end{array}$ & $\begin{array}{l}\text { Northing } \\
\text { (UTM) }\end{array}$ & \begin{tabular}{|c|} 
Land-surface \\
Altitude \\
(meters)
\end{tabular} & $\begin{array}{c}\text { Mean Water- } \\
\text { Level Altitude } \\
\text { (meters) }\end{array}$ \\
\hline 363519116322001 & PW-11 & 541518 & 4049937 & 737.0 & 694.3 \\
\hline 363540116240801 & PW-12 & 553471 & 4049848 & 771.1 & 722.1 \\
\hline 363527116292501 & PW-13 & 545596 & 4049403 & 744.0 & 697.8 \\
\hline 363521116352501 & PW-14 & 536552 & 4049329 & 730.1 & 690.1 \\
\hline 363456116335501 & PW-15 & 538889 & 4049000 & 740.7 & 707.4 \\
\hline 363454116314201 & PW-16 & 542194 & 4048892 & 733.7 & 694.1 \\
\hline 363503116351501 & PW-17 & 536903 & 4048621 & 727.9 & 691.0 \\
\hline 363503116284001 & PW-18 & 546718 & 4048669 & 740.7 & 693.6 \\
\hline 363436116342301 & PW-19 & 538196 & 4048442 & 740.7 & 706.9 \\
\hline 363436116333201 & PW-20 & 540035 & 4048450 & 731.5 & 693.7 \\
\hline 363434116354001 & PW-21 & 536655 & 4048405 & 727.1 & 690.2 \\
\hline 363438116324601 & PW-22 & 540608 & 4048083 & 727.9 & 695.2 \\
\hline 363442116363301 & PW-23 & 534967 & 4047966 & 725.7 & 689.2 \\
\hline 363440116282401 & PW-24 & 547120 & 4047963 & 731.5 & 686.4 \\
\hline 363415116275101 & PW-25 & 547941 & 4047782 & 741.9 & 696.2 \\
\hline 363407116342501 & PW-26 & 537727 & 4047670 & 723.3 & 691.4 \\
\hline 363407116243501 & PW-27 & 552390 & 4047685 & 762.0 & 709.0 \\
\hline 363429116315901 & PW-28 & 541778 & 4047596 & 729.1 & 690.4 \\
\hline 363405116321501 & PW-29 & 541381 & 4047563 & 740.7 & 705.6 \\
\hline 363428116240301 & PW-30 & 553609 & 4047631 & 754.3 & 720.1 \\
\hline 363428116234701 & PW-31 & 554006 & 4047633 & 755.2 & 718.9 \\
\hline 363417116271801 & Nye County Land Company & 548466 & 4047261 & 740.7 & 690.1 \\
\hline 363411116272901 & Amargosa Town Complex & 548492 & 4047077 & 739.1 & 688.8 \\
\hline 363410116261101 & Nye County Development Co. & 550431 & 4047057 & 743.7 & 691.2 \\
\hline 363410116240301 & PW-32 & 553612 & 4047076 & 748.6 & 717.4 \\
\hline 363410116240001 & PW-33 & 553687 & 4047077 & 749.8 & 714.8 \\
\hline 363407116273301 & Amargosa Valley Water & 548393 & 4046953 & 737.9 & 701.3 \\
\hline 363342116335701 & PW-34 & 539147 & 4046844 & 723.9 & 696.5 \\
\hline 363340116332901 & PW-35 & 539968 & 4046817 & 724.2 & 694.2 \\
\hline 363342116325101 & PW-36 & 540788 & 4046821 & 724.2 & 694.0 \\
\hline 363350116252101 & PW-37 & 552097 & 4046882 & 746.8 & 699.5 \\
\hline 365157116271202 & USW H-1 tube 1 & 548727 & 4079926 & 1303.0 & 785.5 \\
\hline 365157116271203 & USW H-1 tube 2 & 548727 & 4079926 & 1303.0 & 736.0 \\
\hline 365157116271204 & USW H-1 tube 3 & 548727 & 4079926 & 1303.0 & 730.6 \\
\hline 365157116271205 & USW H-1 tube 4 & 548727 & 4079926 & 1303.0 & 730.8 \\
\hline 365122116275502 & USW H-5 upper & 547668 & 4078841 & 1478.9 & 775.5 \\
\hline 365122116275503 & USW H-5 lower & 547668 & 4078841 & 1478.9 & 775.6 \\
\hline 365108116262302 & UE-25 b\#1 lower & 549949 & 4078423 & 1200.7 & 729.7 \\
\hline 365108116262303 & UE-25 b\#1 upper & 549949 & 4078423 & 1200.7 & 730.6 \\
\hline 365049116285502 & USW H-6 upper & 546188 & 4077816 & 1301.8 & 776.0 \\
\hline
\end{tabular}


Table A-1. Easting, Northing, Land Surface Altitude, Mean Water-Level Altitude (DTN: GS010908312332.002) (Continued)

\begin{tabular}{|c|c|c|c|c|c|}
\hline USGS Site ID & Site Name & $\begin{array}{l}\text { Easting } \\
\text { (UTM) }\end{array}$ & $\begin{array}{c}\text { Northing } \\
\text { (UTM) }\end{array}$ & \begin{tabular}{|c|} 
Land-surface \\
Altitude \\
(meters)
\end{tabular} & $\begin{array}{c}\begin{array}{c}\text { Mean Water- } \\
\text { Level Altitude } \\
\text { (meters) }\end{array} \\
\end{array}$ \\
\hline 365049116285505 & USW H-6 lower & 546188 & 4077816 & 1301.8 & 775.9 \\
\hline 365032116265402 & USW H-4 upper & 549188 & 4077309 & 1248.5 & 730.4 \\
\hline 365032116265403 & USW H-4 lower & 549188 & 4077309 & 1248.5 & 730.5 \\
\hline 364942116280002 & USW H-3 upper & 547562 & 4075759 & 1483.2 & 731.5 \\
\hline 364942116280003 & USW H-3 lower & 547562 & 4075759 & 1483.2 & 755.9 \\
\hline 364938116252102 & UE-25 p\#1 (Lwr Intrvl) & 551501 & 4075659 & 1114.2 & 752.4 \\
\hline not available yet & USW SD-7 & 548384 & 4076499 & 1363.1 & 727.6 \\
\hline not available yet & USW SD-9 & 548550 & 4079257 & 1303.4 & 731.1 \\
\hline not available yet & USW SD-12 & 548492 & 4077415 & 1323.7 & 730.0 \\
\hline 364234116351501 & NC-EWDP-1DX, shallow zone & 536848 & 4062508 & 803.6 & 786.8 \\
\hline 364234116351501 & NC-EWDP-1DX, deep zone & 536848 & 4062508 & 803.6 & 748.8 \\
\hline 364233116351501 & NC-EWDP-1S, probe 1 & 536851 & 4062504 & 803.8 & 787.1 \\
\hline 364233116351501 & NC-EWDP-1S, probe 2 & 536851 & 4062504 & 803.8 & 786.8 \\
\hline 363939116275401 & NC-EWDP-2D & 547823 & 4057170 & 801.2 & 706.1 \\
\hline 363940116275501 & NC-EWDP-2DB & 547800 & 4057195 & 801.3 & 713.3 \\
\hline 364054116321401 & NC-EWDP-3D & 541352 & 4059450 & 799.4 & 718.3 \\
\hline 364054116321301 & NC-EWDP-3S, probe 2 & 541349 & 4059450 & 798.8 & 719.8 \\
\hline 364332116332201 & NC-EWDP-7S & 539638 & 4064323 & 836.9 & 830.1 \\
\hline 364145116334401 & NC-EWDP-9SX, probe 1 & 539118 & 4061010 & 797.3 & 766.7 \\
\hline 364145116334401 & NC-EWDP-9SX, probe 2 & 539118 & 4061010 & 797.3 & 767.3 \\
\hline 364145116334401 & NC-EWDP-9SX, probe 4 & 539118 & 4061010 & 797.3 & 766.8 \\
\hline 364137116351001 & NC-EWDP-12PA & 536985 & 4060772 & 774.7 & 722.9 \\
\hline 364138116351001 & NC-EWDP-12PB & 536952 & 4060799 & 774.7 & 723.0 \\
\hline 364139116351001 & NC-EWDP-12PC & 536951 & 4060814 & 774.5 & 720.7 \\
\hline 364011116294901 & NC-EWDP-15P & 544927 & 4058163 & 786.9 & 722.5 \\
\hline 364015116265301 & NC-EWDP-19P & 549329 & 4058292 & 819.2 & 707.5 \\
\hline 364014116265301 & NC-EWDP-19D & 549317 & 4058270 & 819.2 & 712.8 \\
\hline 365301116271301 & USW WT-24 & 548697 & 4081910 & 1493.6 & 840.1 \\
\hline 363951116252401 & NC-Washburn-1X & 551545 & 4057569 & 824.1 & 714.6 \\
\hline 364706116170601 & UE-25 J-11 & 563799 & 4071058 & 1049.5 & 732.2 \\
\hline 364237116365401 & BGMW-11 & 534386 & 4062600 & 787.9 & 715.9 \\
\hline 363709116264601 & PW-38 & 549529 & 4052567 & 775.7 & 704.0 \\
\hline 363409116233701 & PW-39 & 551348 & 4047432 & 755.9 & 713.2 \\
\hline 363411116264701 & PW-40 & 549532 & 4047668 & 745.2 & 689.5 \\
\hline 363428116281201 & Amargosa Water & 547420 & 4047594 & 738.2 & 690.4 \\
\hline 363429116233401 & PW-41 & 554329 & 4047666 & 755.3 & 715.7 \\
\hline 363511116335101 & PW-42 & 538989 & 4048877 & 729.4 & 690.8 \\
\hline 365624116222901 & USW UZ-N91 & 555680 & 4088196 & 1203.0 & 1186.7 \\
\hline
\end{tabular}




\section{A2. TABLE A-2: NUMBER OF DATA POINTS USED, SOURCE, AND USE}

\section{Number of Data Points Used}

The number of data points used to determine the mean in Table A-1.

\section{Source}

The DTN for the source from which the water-level data was used to determine the mean (Table A-1) and minimum and maximum (Table A-4) water-level altitude.

\section{Use}

The most appropriate use for each water level was identified as:

- Potentiometric-surface map and calibration (WT).

- Calibration (C).

- Assumed to be perched (P). These water-level observations are not considered in construction of the potentiometric-surface map nor considered as calibration points.

- Unreliable (U) and therefore not used in the construction of the potentiometric-surface map. These water-level observations are not recommended for use in SZ site-scale model calibration.

A water-level measurement was identified as applicable for potentiometric-surface map construction if it was:

- The water level from the upper interval (or only interval) from a borehole

- The water-level interval in the shallow (uppermost) aquifer system, typically the volcanic- or alluvial-aquifer system.

A water-level measurement was identified as unreliable on the basis of the criteria listed in Table A-3. In addition, the following boreholes contained in the Appendix were excluded from this analysis for the reasons stated below:

- Boreholes PW-32 and PW-33 (ID numbers 363410116240301 and 363410116240001 ): The data for these boreholes consist of two water-level measurements in each borehole that span more than 20 years and differ by $6.9 \mathrm{~m}$ and $17.7 \mathrm{~m}$, respectively. An average of the two values for each of these boreholes does not produce a water level that is representative of the early 1990s. The average is considered "Unreliable." There are other boreholes nearby that have reliable water levels that are sufficient for the SZ site-scale modeling. 
- PW-2 well: Similar arguments apply here as for Boreholes PW-32 and PW-33. Water-level data from this borehole are not critical for model calibration because there are other boreholes nearby that have reliable water levels.

- PW-9 well: Similar arguments apply as for Boreholes PW-32 and PW-33. Water-level data from this borehole are not critical for model calibration because there are other boreholes nearby that have reliable water levels.

The remaining water-level data were labeled as suitable for calibration only. Calibration of the SZ site-scale model should be based on all water-level data except those labeled as unreliable (U).

\section{Clarification}

The data provided in DTN: GS991100002330.001 [DIRS 122818] are located in one of three records packages. Data for most wells are located in records package MOL.20000425.0964. Data for wells UE-29 a\#2 and USW UZ-N91 are in records package MOL.20000609.0113. One additional data point for each of the two wells UE-25 a\#1 and UE-25 c\#1 is given in records package MOL.20000919.0380.

Well UE-25 c\#3 - Data from four different source DTNs were combined. For DTN: GS000408312312.001 [DIRS 148696], only the four data points from the upper interval were used in the analysis. The other data from this DTN were not from the same interval as the later DTNs and could not be used.

Table A-2. Number of Data Points Used, Source, and Use

\begin{tabular}{|l|l|l|l|l|}
\hline \multicolumn{1}{|c|}{ USGS Site ID } & \multicolumn{1}{|c|}{ Site Name } & \multicolumn{1}{c|}{$\begin{array}{c}\text { Number of } \\
\text { Data Points Used }\end{array}$} & \multicolumn{1}{c|}{ Source } & \multicolumn{1}{c|}{ Use } \\
\hline 365629116222602 & UE-29 a\#2 & 208 & GS991100002330.001 & WT \\
\hline 365520116370301 & GEXA Well 4 & 52 & GS991100002330.001 & WT \\
\hline 365340116264601 & UE-25 WT\#6 & 117 & GS960908312312.010 & P \\
\hline 365322116273501 & USW G-2 & 28 & GS960908312312.010 & P \\
\hline 365239116253401 & UE-25 WT\#16 & 123 & GS960908312312.010 & WT \\
\hline 365208116274001 & USW UZ-14 & Estimate & GS950508312312.005 & WT \\
\hline 365207116264201 & UE-25 WT\#18 & 38 & GS960908312312.010 & WT \\
\hline 365200116272901 & USW G-1 & 1 & GS991100002330.001 & WT \\
\hline 365147116185301 & UE-25 a\#3 & 1 & GS991100002330.001 & WT \\
\hline 365140116260301 & UE-25 WT\#4 & 131 & GS960908312312.010 & WT \\
\hline 365116116233801 & UE-25 WT\#15 & 124 & GS960908312312.010 & WT \\
\hline 365114116270401 & USW G-4 & 29 & GS931008312312.025 & WT \\
& & & GS970600012847.001 & \\
\hline 365105116262401 & UE-25 a\#1 & 40 & GS991100002330.001 & \\
\hline 365032116243501 & UE-25 WT\#14 & 135 & GS960908312312.010 & WT \\
\hline 365023116271801 & USW WT-2 & 106 & GS960908312312.010 & WT \\
\hline
\end{tabular}


Table A-2. Number of Data Points Used, Source, and Use (Continued)

\begin{tabular}{|c|c|c|c|c|}
\hline USGS Site ID & Site Name & $\begin{array}{c}\text { Number of } \\
\text { Data Points Used }\end{array}$ & Source & Use \\
\hline 364947116254300 & UE-25 c\#1 & 3 & GS991100002330.001 & WT \\
\hline 364947116254501 & UE-25 c\#3 & 8 & $\begin{array}{l}\text { GS960208312312.003 } \\
\text { GS000608312312.004 } \\
\text { GS000708312312.005 } \\
\text { GS000408312312.001 }\end{array}$ & WT \\
\hline 364947116254401 & UE-25 c\#2 & 10 & $\begin{array}{l}\text { GS930408312312.015 } \\
\text { GS000608312312.004 } \\
\text { GS000708312312.005 } \\
\text { GS000408312312.001 } \\
\text { GS950108312312.001 }\end{array}$ & WT \\
\hline 364945116235001 & UE-25 WT\#13 & 118 & GS960908312312.010 & WT \\
\hline 364933116285701 & USW WT- 7 & 113 & GS960908312312.010 & WT \\
\hline 364916116265601 & USW WT- 1 & 128 & GS960908312312.010 & WT \\
\hline 364905116280101 & USW G-3 & 113 & GS960908312312.010 & WT \\
\hline 364828116234001 & UE-25 J-13 & 121 & GS960908312312.010 & WT \\
\hline 364825116290501 & USW WT-10 & 132 & GS960908312312.010 & WT \\
\hline 364822116262601 & UE-25 WT\#17 & 117 & GS960908312312.010 & WT \\
\hline 365821116343701 & USW VH-2 & 1 & GS991100002330.001 & WT \\
\hline 364757116245801 & UE-25 WT\#3 & 119 & GS960908312312.010 & WT \\
\hline 364732116330701 & USW VH-1 & 147 & GS960908312312.010 & WT \\
\hline 364656116261601 & UE-25 WT\#12 & 123 & GS960908312312.010 & WT \\
\hline 364649116280201 & USW WT-11 & 119 & GS960908312312.010 & WT \\
\hline 364554116232400 & UE-25 J-12 & 100 & GS960908312312.010 & WT \\
\hline 364528116232201 & UE-25 JF\#3 & 234 & GS991100002330.001 & WT \\
\hline 364105116302601 & Cind-R-Lite Well & 62 & GS991100002330.001 & WT \\
\hline 363907116235701 & PW-1 & 1 & GS991100002330.001 & $\mathrm{U}$ \\
\hline 363836116234001 & PW-2 & 2 & GS991100002330.001 & $U$ \\
\hline 363840116235000 & PW-3 & 1 & GS991100002330.001 & $U$ \\
\hline 363840116234001 & PW-4 & 1 & GS991100002330.001 & $U$ \\
\hline 363840116233501 & PW-5 & 1 & GS991100002330.001 & $U$ \\
\hline 363835116234001 & NDOT Well & 87 & GS991100002330.001 & WT \\
\hline 363742116263201 & PW-6 & 3 & GS991100002330.001 & WT \\
\hline 363830116241401 & Airport Well & 90 & GS991100002330.001 & WT \\
\hline 363815116175901 & TW- 5 & 99 & GS991100002330.001 & WT \\
\hline 363711116263701 & PW-7 & 4 & GS991100002330.001 & $U$ \\
\hline 363621116263201 & PW-8 & 1 & GS991100002330.001 & $U$ \\
\hline 363549116305001 & Nye County Development Co. & 3 & GS991100002330.001 & WT \\
\hline 363523116353701 & PW-9 & 3 & GS991100002330.001 & $U$ \\
\hline 363525116325601 & PW-10 & 6 & GS991100002330.001 & WT \\
\hline 363519116322001 & PW-11 & 4 & GS991100002330.001 & WT \\
\hline 363540116240801 & PW-12 & 22 & GS991100002330.001 & WT \\
\hline 363527116292501 & PW-13 & 2 & GS991100002330.001 & WT \\
\hline 363521116352501 & PW-14 & 63 & GS991100002330.001 & WT \\
\hline
\end{tabular}


Table A-2. Number of Data Points Used, Source, and Use (Continued)

\begin{tabular}{|c|c|c|c|c|}
\hline USGS Site ID & Site Name & $\begin{array}{c}\text { Number of } \\
\text { Data Points Used }\end{array}$ & Source & Use \\
\hline 363456116335501 & PW-15 & 4 & GS9911000002330.001 & $\mathrm{U}$ \\
\hline 363454116314201 & PW-16 & 4 & GS991100002330.001 & WT \\
\hline 363503116351501 & PW-17 & 3 & GS991100002330.001 & WT \\
\hline 363503116284001 & PW-18 & 2 & GS991100002330.001 & WT \\
\hline 363436116342301 & PW-19 & 5 & GS991100002330.001 & WT \\
\hline 363436116333201 & PW-20 & 4 & GS991100002330.001 & WT \\
\hline 363434116354001 & PW-21 & 19 & GS991100002330.001 & WT \\
\hline 363438116324601 & PW-22 & 4 & GS991100002330.001 & WT \\
\hline 363442116363301 & PW-23 & 1 & GS991100002330.001 & WT \\
\hline 363440116282401 & PW-24 & 1 & GS991100002330.001 & $\mathrm{U}$ \\
\hline 363415116275101 & PW-25 & 1 & GS991100002330.001 & $\mathrm{U}$ \\
\hline 363407116342501 & PW-26 & 3 & GS991100002330.001 & WT \\
\hline 363407116243501 & PW-27 & 1 & GS991100002330.001 & $\mathrm{U}$ \\
\hline 363429116315901 & PW-28 & 3 & GS991100002330.001 & WT \\
\hline 363405116321501 & PW-29 & 2 & GS991100002330.001 & $\mathrm{U}$ \\
\hline 363428116240301 & PW-30 & 3 & GS991100002330.001 & WT \\
\hline 363428116234701 & PW-31 & 88 & GS991100002330.001 & WT \\
\hline 363417116271801 & Nye County Land Company & 2 & GS991100002330.001 & WT \\
\hline 363411116272901 & Amargosa Town Complex & 1 & GS991100002330.001 & WT \\
\hline 363410116261101 & Nye County Development Co. & 1 & GS9911000002330.001 & WT \\
\hline 363410116240301 & PW-32 & 2 & GS991100002330.001 & $U$ \\
\hline 363410116240001 & PW-33 & 2 & GS991100002330.001 & U \\
\hline 363407116273301 & Amargosa Valley Water & 1 & GS991100002330.001 & WT \\
\hline 363342116335701 & PW-34 & 1 & GS991100002330.001 & $\mathrm{U}$ \\
\hline 363340116332901 & PW-35 & 48 & GS991100002330.001 & WT \\
\hline 363342116325101 & PW-36 & 46 & GS991100002330.001 & WT \\
\hline 363350116252101 & PW-37 & 2 & GS991100002330.001 & $\mathrm{U}$ \\
\hline 365157116271202 & USW $\mathrm{H}-1$ tube 1 & 101 & GS960908312312.010 & C \\
\hline 365157116271203 & USW H-1 tube 2 & 75 & GS960908312312.010 & C \\
\hline 365157116271204 & USW $\mathrm{H}-1$ tube 3 & 108 & GS960908312312.010 & C \\
\hline 365157116271205 & USW H-1 tube 4 & 124 & GS960908312312.010 & WT \\
\hline 365122116275502 & USW H-5 upper & 106 & GS960908312312.010 & WT \\
\hline 365122116275503 & USW H-5 lower & 54 & GS960908312312.010 & C \\
\hline 365108116262302 & UE-25 b\#1 lower & 67 & GS960908312312.010 & C \\
\hline 365108116262303 & UE-25 b\#1 upper & 99 & GS960908312312.010 & WT \\
\hline \begin{tabular}{|l|}
365049116285502 \\
\end{tabular} & USW H-6 upper & 118 & GS960908312312.010 & WT \\
\hline 365049116285505 & USW H-6 lower & 79 & GS960908312312.010 & C \\
\hline 365032116265402 & USW H-4 upper & 128 & GS960908312312.010 & WT \\
\hline 365032116265403 & USW H-4 lower & 101 & GS960908312312.010 & C \\
\hline 364942116280002 & USW H-3 upper & 128 & GS960908312312.010 & WT \\
\hline
\end{tabular}


Table A-2. Number of Data Points Used, Source, and Use (Continued)

\begin{tabular}{|c|c|c|c|c|}
\hline USGS Site ID & Site Name & $\begin{array}{c}\text { Number of } \\
\text { Data Points Used }\end{array}$ & Source & Use \\
\hline 364942116280003 & USW H-3 lower & 59 & $\begin{array}{l}\text { GS960908312312.010 } \\
\text { GS980308312312.004 }\end{array}$ & C \\
\hline 364938116252102 & UE-25 p\#1(Lwr Intrvl) & 120 & GS960908312312.010 & C \\
\hline not available & USW SD-7 & 1 & $\begin{array}{l}\text { TM0000SD7SUPER.001: } \\
\text { DRC.19960926.0376 } \\
\text { page } 234\end{array}$ & $U$ \\
\hline not available & USW SD-9 & 1 & $\begin{array}{l}\text { TM0000SD9SUPER.002: } \\
\text { DRC.19960819.0101 } \\
\text { page } 88\end{array}$ & WT \\
\hline not available & USW SD-12 & 1 & $\begin{array}{l}\text { TM000SD12SUPER.003: } \\
\text { DRC.19960926.0180 } \\
\text { page } 207\end{array}$ & WT \\
\hline 364234116351501 & NC-EWDP-1DX, shallow & 14 & $\begin{array}{l}\text { MO0112DQRWLNYE.024 } \\
\text { MO0112DQRWLNYE.017 }\end{array}$ & WT \\
\hline 364234116351501 & NC-EWDP-1DX, deep & 13 & $\begin{array}{l}\text { MO0112DQRWLNYE.024 } \\
\text { MO0112DQRWLNYE.017 }\end{array}$ & C \\
\hline 364233116351501 & NC-EWDP-1S, probe 1 & 26816 & $\begin{array}{l}\text { MO0112DQRWLNYE.019 } \\
\text { MO0111DQRWLNYE.002 } \\
\text { MO0112DQRWLNYE.023 }\end{array}$ & WT \\
\hline 364233116351501 & NC-EWDP-1S, probe 2 & 18783 & $\begin{array}{l}\text { MO0112DQRWLNYE.019 } \\
\text { MO0111DQRWLNYE.002 } \\
\text { MO0112DQRWLNYE.023 }\end{array}$ & C \\
\hline 363939116275401 & NC-EWDP-2D & 2 & $\begin{array}{l}\text { MO0112DQRWLNYE.024 } \\
\text { MO0112DQRWLNYE.020 }\end{array}$ & WT \\
\hline 363940116275501 & NC-EWDP-2DB & 2 & $\begin{array}{l}\text { MO0112DQRWLNYE.025 } \\
\text { MO0112DQRWLNYE.020 }\end{array}$ & C \\
\hline 364054116321401 & NC-EWDP-3D & 30 & $\begin{array}{l}\text { MO0112DQRWLNYE.024 } \\
\text { MO0112DQRWLNYE.016 }\end{array}$ & C \\
\hline 364054116321301 & NC-EWDP-3S, probe 2 & 27991 & $\begin{array}{l}\text { MO0112DQRWLNYE.019 } \\
\text { MO0112DQRWLNYE.015 } \\
\text { MO0112DQRWLNYE.022 } \\
\text { MO0111DQRWLNYE.003 }\end{array}$ & WT \\
\hline 364054116321301 & NC-EWDP-3S, probe 3 & 32124 & $\begin{array}{l}\text { MO0112DQRWLNYE.019 } \\
\text { MO0112DQRWLNYE.015 } \\
\text { MO0111DQRWLNYE.003 } \\
\text { MO0112DQRWLNYE.022 }\end{array}$ & C \\
\hline 363925116241501 & NC-EWDP-4PA & 14 & $\begin{array}{l}\text { MO0112DQRWLNYE.025 } \\
\text { MO0112DQRWLNYE.005 }\end{array}$ & WT \\
\hline 363925116241401 & NC-EWDP-4PB & 6 & $\begin{array}{l}\text { MO0112DQRWLNYE.025 } \\
\text { MO0112DQRWLNYE.006 }\end{array}$ & C \\
\hline 364012116223401 & NC-EWDP-5SB & 11 & $\begin{array}{l}\text { MO0112DQRWLNYE.025 } \\
\text { MO0112DQRWLNYE.008 }\end{array}$ & WT \\
\hline 364332116332201 & NC-EWDP-7S & 3 & $\begin{array}{l}\text { MO0112DQRWLNYE.025 } \\
\text { MO0112DQRWLNYE.007 }\end{array}$ & $P$ \\
\hline
\end{tabular}


Table A-2. Number of Data Points Used, Source, and Use (Continued)

\begin{tabular}{|c|c|c|c|c|}
\hline USGS Site ID & Site Name & $\begin{array}{c}\text { Number of } \\
\text { Data Points Used }\end{array}$ & Source & Use \\
\hline 364145116334401 & NC-EWDP-9SX, probe 1 & 29059 & $\begin{array}{l}\text { MO0112DQRWLNYE.019 } \\
\text { MO0111DQRWLNYE.004 } \\
\text { MO0112DQRWLNYE.021 } \\
\text { MO0112DQRWLNYE.011 }\end{array}$ & WT \\
\hline 364145116334401 & NC-EWDP-9SX, probe 2 & 38706 & $\begin{array}{l}\text { MO0112DQRWLNYE.019 } \\
\text { MO0111DQRWLNYE.004 } \\
\text { MO0112DQRWLNYE.021 } \\
\text { MO0112DQRWLNYE.011 }\end{array}$ & C \\
\hline 364145116334401 & NC-EWDP-9SX, probe 4 & 38560 & $\begin{array}{l}\text { MO0112DQRWLNYE.019 } \\
\text { MO0111DQRWLNYE.004 } \\
\text { MO0112DQRWLNYE.021 } \\
\text { MO0112DQRWLNYE.011 }\end{array}$ & C \\
\hline 364137116351001 & NC-EWDP-12PA & 25 & $\begin{array}{l}\text { MO0112DQRWLNYE.025 } \\
\text { MO0112DQRWLNYE.010 }\end{array}$ & C \\
\hline 364138116351001 & NC-EWDP-12PB & 25 & $\begin{array}{l}\text { MO0112DQRWLNYE.025 } \\
\text { MO0112DQRWLNYE.012 }\end{array}$ & C \\
\hline 364139116351001 & NC-EWDP-12PC & 26 & $\begin{array}{l}\text { MO0112DQRWLNYE.025 } \\
\text { MO0112DQRWLNYE.013 }\end{array}$ & WT \\
\hline 364011116294901 & NC-EWDP-15P & 12 & $\begin{array}{l}\text { MO0112DQRWLNYE.025 } \\
\text { MO0112DQRWLNYE.009 }\end{array}$ & WT \\
\hline 364015116265301 & NC-EWDP-19P & 9 & $\begin{array}{l}\text { MO0112DQRWLNYE.025 } \\
\text { MO0112DQRWLNYE.014 }\end{array}$ & WT \\
\hline 364014116265301 & NC-EWDP-19D & 5 & $\begin{array}{l}\text { MO0112DQRWLNYE.025 } \\
\text { MO0112DQRWLNYE.018 }\end{array}$ & C \\
\hline 365301116271301 & USW WT-24 & 11 & $\begin{array}{l}\text { GS990908312312.005 } \\
\text { GS000608312312.003 } \\
\text { GS000808312312.007 } \\
\end{array}$ & WT \\
\hline 363951116252401 & NC-Washburn-1X & 59 & MO0112DQRWLNYE.024 & WT \\
\hline 364706116170601 & UE-25 J-11 & 71 & GS960908312312.010 & WT \\
\hline 364237116365401 & BGMW-11 & 51 & GS991100002330.001 & WT \\
\hline 363709116264601 & PW-38 & 1 & GS991100002330.001 & WT \\
\hline 363409116233701 & PW-39 & 1 & GS991100002330.001 & U \\
\hline 363411116264701 & PW-40 & 1 & GS991100002330.001 & WT \\
\hline 363428116281201 & Amargosa Water & 1 & GS991100002330.001 & WT \\
\hline 363429116233401 & PW-41 & 1 & GS991100002330.001 & WT \\
\hline 363511116335101 & PW-42 & 1 & GS991100002330.001 & WT \\
\hline 365624116222901 & USW UZ-N91 & 209 & GS991100002330.001 & WT \\
\hline
\end{tabular}




\section{A3. TABLE A-3: RELIABILITY OF MEASUREMENTS}

\section{Reliability of Measurements}

Using professional judgment, an assessment of the overall reliability of the average water-level data to represent 1990's water levels (Table A-1) was made. The following categories were assigned:

- Best (average water level documented by Graves et al. 1997 [DIRS 10146]; DTN: GS960908312312.010)

- Reliable (all others not identified in the other three categories)

- Less Reliable (fewer than 5 water-level measurements) or (latest measurement made prior to 1980) or (only data point available within a 5-km radius)

- Unreliable (fewer than 5 measurements, all made prior to 1980).

Table A-3. Reliability of Measurements

\begin{tabular}{|c|c|c|}
\hline USGS Site ID & Site Name & Reliability of Measurements \\
\hline 365629116222602 & UE-29 a\#2 & Reliable \\
\hline 365520116370301 & GEXA Well 4 & Reliable \\
\hline 365340116264601 & UE-25 WT\#6 & Best \\
\hline 365322116273501 & USW G-2 & Best \\
\hline 365239116253401 & UE-25 WT\#16 & Best \\
\hline 365208116274001 & USW UZ-14 & Less Reliable (fewer than 5 measurements) \\
\hline 365207116264201 & UE-25 WT\#18 & Best \\
\hline 365200116272901 & USW G-1 & Less Reliable (fewer than 5 measurements) \\
\hline 365147116185301 & UE-25 a\#3 & Less Reliable (only data points within 5-km radius) \\
\hline 365140116260301 & UE-25 WT\#4 & Best \\
\hline 365116116233801 & UE-25 WT\#15 & Best \\
\hline 365114116270401 & USW G-4 & Reliable \\
\hline 365105116262401 & UE-25 a\#1 & Reliable \\
\hline 365032116243501 & UE-25 WT\#14 & Best \\
\hline 365023116271801 & USW WT-2 & Best \\
\hline 364947116254300 & UE-25 c\#1 & Less Reliable (fewer than 5 measurements) \\
\hline 364947116254501 & UE-25 c\#3 & Reliable \\
\hline 364947116254401 & UE-25 c\#2 & Reliable \\
\hline 364945116235001 & UE-25 WT\#13 & Best \\
\hline 364933116285701 & USW WT-7 & Best \\
\hline 364916116265601 & USW WT-1 & Best \\
\hline 364905116280101 & USW G-3 & Best \\
\hline 364828116234001 & UE-25 J-13 & Best \\
\hline 364825116290501 & USW WT-10 & Best \\
\hline 364822116262601 & UE-25 WT\#17 & Best \\
\hline
\end{tabular}


Table A-3. Reliability of Measurements (Continued)

\begin{tabular}{|c|c|c|}
\hline USGS Site ID & Site Name & Reliability of Measurements \\
\hline 365821116343701 & USW VH-2 & Less Reliable (fewer than 5 measurements) \\
\hline 364757116245801 & UE-25 WT\#3 & Best \\
\hline 364732116330701 & USW VH-1 & Best \\
\hline 364656116261601 & UE-25 WT\#12 & Best \\
\hline 364649116280201 & USW WT-11 & Best \\
\hline 364554116232400 & UE-25 J-12 & Best \\
\hline 364528116232201 & UE-25 JF\#3 & Reliable \\
\hline 364105116302601 & Cind-R-Lite Well & Reliable \\
\hline 363907116235701 & PW-1 & Unreliable (fewer than 5 measurements before 1980) \\
\hline 363836116234001 & PW-2 & Unreliable (fewer than 5 measurements before 1980) \\
\hline 363840116235000 & PW-3 & Unreliable (fewer than 5 measurements before 1980) \\
\hline 363840116234001 & PW-4 & Unreliable (fewer than 5 measurements before 1980) \\
\hline 363840116233501 & PW-5 & Unreliable (fewer than 5 measurements before 1980) \\
\hline 363835116234001 & NDOT Well & Reliable \\
\hline 363742116263201 & PW-6 & Less Reliable (fewer than 5 measurements) \\
\hline 363830116241401 & Airport Well & Reliable \\
\hline 363815116175901 & TW- 5 & Reliable \\
\hline 363711116263701 & PW-7 & Unreliable (fewer than 5 measurements before 1980) \\
\hline 363621116263201 & PW-8 & Unreliable (fewer than 5 measurements before 1980) \\
\hline 363549116305001 & Nye County Development Co. & Less Reliable (fewer than 5 measurements) \\
\hline 363523116353701 & PW-9 & Unreliable (fewer than 5 measurements before 1980) \\
\hline 363525116325601 & PW-10 & Reliable \\
\hline 363519116322001 & PW-11 & Less Reliable (fewer than 5 measurements) \\
\hline 363540116240801 & PW-12 & Less Reliable (latest measurement prior to 1980) \\
\hline 363527116292501 & PW-13 & Less Reliable (fewer than 5 measurements) \\
\hline 363521116352501 & PW-14 & Reliable \\
\hline 363456116335501 & PW-15 & Unreliable (fewer than 5 measurements before 1980) \\
\hline 363454116314201 & PW-16 & Reliable \\
\hline 363503116351501 & PW-17 & Less Reliable (fewer than 5 measurements) \\
\hline 363503116284001 & PW-18 & Less Reliable (fewer than 5 measurements) \\
\hline 363436116342301 & PW-19 & Reliable \\
\hline 363436116333201 & PW-20 & Reliable \\
\hline 363434116354001 & PW-21 & Reliable \\
\hline 363438116324601 & PW-22 & Less Reliable (fewer than 5 measurements) \\
\hline 363442116363301 & PW-23 & Less Reliable (fewer than 5 measurements) \\
\hline 363440116282401 & PW-24 & Unreliable (fewer than 5 measurements before 1980) \\
\hline 363415116275101 & PW-25 & Unreliable (fewer than 5 measurements before 1980) \\
\hline 363407116342501 & PW-26 & Less Reliable (fewer than 5 measurements) \\
\hline 363407116243501 & PW-27 & Unreliable (fewer than 5 measurements before 1980) \\
\hline 363429116315901 & PW-28 & Less Reliable (fewer than 5 measurements) \\
\hline 363405116321501 & PW-29 & Unreliable (fewer than 5 measurements before 1980) \\
\hline 363428116240301 & PW-30 & Less Reliable (fewer than 5 measurements) \\
\hline 363428116234701 & PW-31 & Reliable \\
\hline
\end{tabular}


Table A-3. Reliability of Measurements (Continued)

\begin{tabular}{|c|c|c|}
\hline USGS Site ID & Site Name & Reliability of Measurements \\
\hline 363417116271801 & Nye County Land Company & Less Reliable (fewer than 5 measurements) \\
\hline 363411116272901 & Amargosa Town Complex & Less Reliable (fewer than 5 measurements) \\
\hline 363410116261101 & Nye County Development Co. & Less Reliable (fewer than 5 measurements) \\
\hline 363410116240301 & PW-32 & Unreliable (fewer than 5 measurements before 1980) \\
\hline 363410116240001 & PW-33 & Unreliable (fewer than 5 measurements before 1980) \\
\hline 363407116273301 & Amargosa Valley Water & Less Reliable (fewer than 5 measurements) \\
\hline 363342116335701 & PW-34 & Unreliable (fewer than 5 measurements before 1980) \\
\hline 363340116332901 & PW-35 & Reliable \\
\hline 363342116325101 & PW-36 & Less Reliable (latest measurement prior to 1980) \\
\hline 363350116252101 & PW-37 & Unreliable (fewer than 5 measurements before 1980) \\
\hline 365157116271202 & USW H-1 tube 1 & Best \\
\hline 365157116271203 & USW H-1 tube 2 & Best \\
\hline 365157116271204 & USW $\mathrm{H}-1$ tube 3 & Best \\
\hline 365157116271205 & USW H-1 tube 4 & Best \\
\hline 365122116275502 & USW H-5 upper & Best \\
\hline 365122116275503 & USW H-5 lower & Best \\
\hline 365108116262302 & UE-25 b\#1 lower & Best \\
\hline 365108116262303 & UE-25 b\#1 upper & Best \\
\hline 365049116285502 & USW H-6 upper & Best \\
\hline 365049116285505 & USW H-6 lower & Best \\
\hline 365032116265402 & USW H-4 upper & Best \\
\hline 365032116265403 & USW H-4 lower & Best \\
\hline 364942116280002 & USW H-3 upper & Best \\
\hline 364942116280003 & USW H-3 lower & Best \\
\hline 364938116252102 & UE-25 p\#1 (Lwr Intrvl) & Best \\
\hline not available yet & USW SD-7 & Less Reliable (fewer than 5 measurements) \\
\hline not available yet & USW SD-9 & Less Reliable (fewer than 5 measurements) \\
\hline not available yet & USW SD-12 & Less Reliable (fewer than 5 measurements) \\
\hline 364234116351501 & NC-EWDP-1DX, shallow & Reliable \\
\hline 364234116351501 & NC-EWDP-1DX, deep & Reliable \\
\hline 364233116351501 & NC-EWDP-1S, probe 1 & Reliable \\
\hline 364233116351501 & NC-EWDP-1S, probe 2 & Reliable \\
\hline 363939116275401 & NC-EWDP-2D & Less Reliable (fewer than 5 measurements) \\
\hline 363940116275501 & NC-EWDP-2DB & Less Reliable (fewer than 5 measurements) \\
\hline 364054116321401 & NC-EWDP-3D & Reliable \\
\hline 364054116321301 & NC-EWDP-3S, probe 2 & Reliable \\
\hline 364054116321301 & NC-EWDP-3S, probe 3 & Reliable \\
\hline 363925116241501 & NC-EWDP-4PA & Reliable \\
\hline 363925116241401 & NC-EWDP-4PB & Reliable \\
\hline 364012116223401 & NC-EWDP-5SB & Reliable \\
\hline 364332116332201 & NC-EWDP-7S & Less Reliable (fewer than 5 measurements) \\
\hline 364145116334401 & NC-EWDP-9SX, probe 1 & Reliable \\
\hline 364145116334401 & NC-EWDP-9SX, probe 2 & Reliable \\
\hline
\end{tabular}


Table A-3. Reliability of Measurements (Continued)

\begin{tabular}{|l|l|l|}
\hline \multicolumn{1}{|c|}{ USGS Site ID } & \multicolumn{1}{|c|}{ Site Name } & \multicolumn{1}{c|}{ Reliability of Measurements } \\
\hline 364145116334401 & NC-EWDP-9SX, probe 4 & Reliable \\
\hline 364137116351001 & NC-EWDP-12PA & Reliable \\
\hline 364138116351001 & NC-EWDP-12PB & Reliable \\
\hline 364139116351001 & NC-EWDP-12PC & Reliable \\
\hline 364011116294901 & NC-EWDP-15P & Reliable \\
\hline 364015116265301 & NC-EWDP-19P & Reliable \\
\hline 364014116265301 & NC-EWDP-19D & Reliable \\
\hline 365301116271301 & USW WT-24 & Reliable \\
\hline 363951116252401 & NC-Washburn-1X & Reliable \\
\hline 364706116170601 & UE-25 J-11 & Best \\
\hline 364237116365401 & BGMW-11 & Reliable \\
\hline 363709116264601 & PW-38 & Less Reliable (fewer than 5 measurements) \\
\hline 363409116233701 & PW-39 & Unreliable (fewer than 5 measurements before 1980) \\
\hline 363411116264701 & PW-40 & Less Reliable (fewer than 5 measurements) \\
\hline 363428116281201 & Amargosa Water & Less Reliable (fewer than 5 measurements) \\
\hline 363429116233401 & PW-41 & Less Reliable (fewer than 5 measurements) \\
\hline 363511116335101 & PW-42 & Less Reliable (fewer than 5 measurements) \\
\hline 365624116222901 & USW UZ-N91 & Reliable \\
\hline
\end{tabular}




\section{A4. TABLE A-4: EARLIEST YEAR OF MEASUREMENT, LATEST YEAR OF MEASUREMENT, MINIMUM WATER-LEVEL ALTITUDE, AND MAXIMUM WATER-LEVEL ALTITUDE (DTN: GS010908312332.002)}

\section{Earliest Year of Measurement/Latest Year of Measurement}

The earliest and latest year of reported measurement used in the calculation of the mean were determined and recorded. The data reported in GS960908312312.010 [DIRS 105063] were collected for the water-level monitoring studies being conducted as part of Yucca Mountain site characterization activities after 1986 under an approved quality assurance program. The data tabulated in GS960908312312.010 [DIRS 105063] were not checked for earlier or later measurements.

\section{Minimum Water-Level Altitude/Maximum Water-Level Altitude (Meters)}

The smallest and largest water-level altitudes for the data used to calculate mean water-level altitude were compiled and tabulated. The altitude was converted from feet to meters by the following formula, where necessary:

$$
\text { Altitude }(\mathrm{ft}) \times 0.3048(\mathrm{~m} / \mathrm{ft})=\text { Altitude (meters) }
$$

The altitude was rounded to the nearest tenth of 1 meter.

Table A-4. Earliest Year of Measurement, Latest Year of Measurement, Minimum Water-Level Altitude, and Maximum Water-Level Altitude (DTN: GS010908312332.002)

\begin{tabular}{|l|l|l|l|l|l|}
\hline USGS Site ID & \multicolumn{1}{|c|}{ Site Name } & \multicolumn{1}{|c|}{$\begin{array}{c}\text { Earliest Year } \\
\text { of Measurement }\end{array}$} & \multicolumn{1}{|c|}{$\begin{array}{c}\text { Latest Year } \\
\text { of Measurement }\end{array}$} & $\begin{array}{c}\text { Maximum } \\
\text { Altitude } \\
\text { (meters) }\end{array}$ & $\begin{array}{c}\text { Water-Level } \\
\text { Altitude } \\
\text { (meters) }\end{array}$ \\
\hline 365629116222602 & UE-29 a\#2 & 1985 & 1996 & 1186.2 & 1191.3 \\
\hline 365520116370301 & GEXA Well 4 & 1989 & 1996 & 995.3 & 1010.1 \\
\hline 365340116264601 & UE-25 WT\#6 & 1985 & 1995 & 1033.3 & 1036.1 \\
\hline 365322116273501 & USW G-2 & 1992 & 1995 & 1019.6 & 1020.6 \\
\hline 365239116253401 & UE-25 WT\#16 & 1985 & 1995 & 737.8 & 738.6 \\
\hline 365208116274001 & USW UZ-14 & N/A & N/A & N/A & N/A \\
\hline 365207116264201 & UE-25 WT\#18 & 1991 & 1995 & 730.5 & 730.9 \\
\hline 365200116272901 & USW G-1 & 1982 & 1982 & 754.2 & 754.2 \\
\hline 365147116185301 & UE-25 a\#3 & 1979 & 1979 & 748.3 & 748.3 \\
\hline 365140116260301 & UE-25 WT\#4 & 1985 & 1995 & 730.3 & 731.2 \\
\hline 365116116233801 & UE-25 WT\#15 & 1985 & 1995 & 729.0 & 729.4 \\
\hline 365114116270401 & USW G-4 & 1983 & 1990 & 730.0 & 730.9 \\
\hline 365105116262401 & UE-25 a\#1 & 1982 & 1985 & 730.7 & 731.2 \\
\hline 365032116243501 & UE-25 WT\#14 & 1985 & 1995 & 729.3 & 730.0 \\
\hline 365023116271801 & USW WT-2 & 1985 & 1995 & 730.1 & 730.8 \\
\hline 364947116254300 & UE-25 C\#1 & 1983 & 1984 & 730.1 & 730.3 \\
\hline 364947116254501 & UE-25 c\#3 & 1989 & 1995 & 730.1 & 730.3 \\
\hline
\end{tabular}


Table A-4. Earliest Year of Measurement, Latest Year of Measurement, Minimum Water-Level Altitude, and Maximum Water-Level Altitude (DTN: GS010908312332.002) (Continued)

\begin{tabular}{|c|c|c|c|c|c|}
\hline USGS Site ID & Site Name & \begin{tabular}{|c|}
$\begin{array}{c}\text { Earliest Year } \\
\text { of Measurement }\end{array}$ \\
\end{tabular} & $\begin{array}{c}\text { Latest Year } \\
\text { of Measurement }\end{array}$ & $\begin{array}{c}\text { Minimum } \\
\text { Water-Level } \\
\text { Altitude } \\
\text { (meters) } \\
\end{array}$ & \begin{tabular}{|c|}
$\begin{array}{c}\text { Maximum } \\
\text { Water-Level } \\
\text { Altitude } \\
\text { (meters) }\end{array}$ \\
\end{tabular} \\
\hline 364947116254401 & UE-25 c\#2 & 1989 & 1995 & 729.9 & 730.3 \\
\hline 364945116235001 & UE-25 WT\#13 & 1985 & 1995 & 728.5 & 729.4 \\
\hline 364933116285701 & USW WT-7 & 1985 & 1995 & 775.5 & 776.0 \\
\hline 364916116265601 & USW WT- 1 & 1985 & 1995 & 730.0 & 730.5 \\
\hline 364905116280101 & USW G-3 & 1985 & 1995 & 730.0 & 730.8 \\
\hline 364828116234001 & UE-25 J-13 & 1986 & 1995 & 728.3 & 728.7 \\
\hline 364825116290501 & USW WT-10 & 1985 & 1995 & 775.6 & 776.2 \\
\hline 364822116262601 & UE-25 WT\#17 & 1985 & 1995 & 729.5 & 729.8 \\
\hline 365821116343701 & USW VH-2 & 1983 & 1983 & 810.4 & 810.4 \\
\hline 364757116245801 & UE-25 WT\#3 & 1985 & 1995 & 729.4 & 729.9 \\
\hline 364732116330701 & USW VH-1 & 1985 & 1995 & 779.3 & 779.6 \\
\hline 364656116261601 & UE-25 WT\#12 & 1985 & 1995 & 729.1 & 729.6 \\
\hline 364649116280201 & USW WT-11 & 1985 & 1995 & 730.2 & 730.8 \\
\hline 364554116232400 & UE-25 J-12 & 1989 & 1995 & 727.8 & 728.2 \\
\hline 364528116232201 & UE-25 JF\#3 & 1992 & 1998 & 727.3 & 728.1 \\
\hline 364105116302601 & Cind-R-Lite Well & 1992 & 1998 & 727.1 & 729.9 \\
\hline 363907116235701 & $\mathrm{PW}-1$ & 1961 & 1961 & 718.4 & 718.4 \\
\hline 363836116234001 & $\mathrm{PW}-2$ & 1964 & 1990 & 700.1 & 705.4 \\
\hline 363840116235000 & $\mathrm{PW}-3$ & 1955 & 1955 & 704.1 & 704.1 \\
\hline 363840116234001 & PW-4 & 1952 & 1952 & 705.6 & 705.6 \\
\hline 363840116233501 & $P W-5$ & 1955 & 1955 & 701.6 & 701.6 \\
\hline 363835116234001 & NDOT Well & 1991 & 1998 & 704.9 & 705.6 \\
\hline 363742116263201 & PW-6 & 1953 & 1987 & 705.4 & 708.1 \\
\hline 363830116241401 & Airport Well & 1987 & 1998 & 705.2 & 705.5 \\
\hline 363815116175901 & TW-5 & 1962 & 1998 & 724.8 & 729.2 \\
\hline 363711116263701 & PW-7 & 1958 & 1962 & 706.1 & 709.3 \\
\hline 363621116263201 & $\mathrm{PW}-8$ & 1958 & 1958 & 704.4 & 704.4 \\
\hline 363549116305001 & $\begin{array}{l}\text { Nye County Development } \\
\text { Co. }\end{array}$ & 1963 & 1987 & 691.3 & 695.9 \\
\hline 363523116353701 & PW-9 & 1960 & 1984 & 688.4 & 694.0 \\
\hline 363525116325601 & $\mathrm{PW}-10$ & 1960 & 1987 & 691.4 & 696.3 \\
\hline 363519116322001 & $\mathrm{PW}-11$ & 1962 & 1987 & 693.5 & 696.1 \\
\hline 363540116240801 & $\mathrm{PW}-12$ & 1963 & 1973 & 721.5 & 726.0 \\
\hline 363527116292501 & $\mathrm{PW}-13$ & 1962 & 1987 & 696.9 & 698.7 \\
\hline 363521116352501 & $\mathrm{PW}-14$ & 1963 & 1998 & 689.7 & 692.0 \\
\hline 363456116335501 & $\mathrm{PW}-15$ & 1961 & 1962 & 707.1 & 707.7 \\
\hline 363454116314201 & PW-16 & 1962 & 1987 & 693.2 & 696.2 \\
\hline 363503116351501 & PW-17 & 1984 & 1987 & 690.5 & 691.3 \\
\hline 363503116284001 & PW-18 & 1965 & 1987 & 692.2 & 694.9 \\
\hline
\end{tabular}


Water-Level Data Analysis for the Saturated Zone Site-Scale Flow and Transport Model

Table A-4. Earliest Year of Measurement, Latest Year of Measurement, Minimum Water-Level Altitude, and Maximum Water-Level Altitude (DTN: GS010908312332.002) (Continued)

\begin{tabular}{|c|c|c|c|c|c|}
\hline USGS Site ID & Site Name & \begin{tabular}{|c|} 
Earliest Year \\
of Measurement
\end{tabular} & \begin{tabular}{|c|} 
Latest Year \\
of Measurement
\end{tabular} & \begin{tabular}{|c|} 
Minimum \\
Water-Level \\
Altitude \\
(meters) \\
\end{tabular} & \begin{tabular}{|c} 
Maximum \\
Water-Level \\
Altitude \\
(meters) \\
\end{tabular} \\
\hline 363436116342301 & PW-19 & 1959 & 1987 & 705.3 & 708.8 \\
\hline 363436116333201 & $\mathrm{PW}-20$ & 1962 & 1987 & 691.5 & 696.0 \\
\hline 363434116354001 & $\mathrm{PW}-21$ & 1987 & 1993 & 689.1 & 690.4 \\
\hline 363438116324601 & $\mathrm{PW}-22$ & 1961 & 1987 & 692.4 & 698.0 \\
\hline 363442116363301 & PW-23 & 1982 & 1982 & 689.2 & 689.2 \\
\hline 363440116282401 & PW-24 & 1962 & 1962 & 686.4 & 686.4 \\
\hline 363415116275101 & $\mathrm{PW}-25$ & 1958 & 1958 & 696.2 & 696.2 \\
\hline 363407116342501 & PW-26 & 1958 & 1984 & 690.1 & 692.8 \\
\hline 363407116243501 & PW-27 & 1962 & 1962 & 709.0 & 709.0 \\
\hline 363429116315901 & PW-28 & 1965 & 1987 & 689.5 & 692.1 \\
\hline 363405116321501 & PW-29 & 1960 & 1962 & 704.7 & 706.6 \\
\hline 363428116240301 & $\mathrm{PW}-30$ & 1987 & 1991 & 719.6 & 720.4 \\
\hline 363428116234701 & $\mathrm{PW}-31$ & 1987 & 1998 & 717.7 & 720.3 \\
\hline 363417116271801 & $\begin{array}{l}\text { Nye County Land } \\
\text { Company }\end{array}$ & 1962 & 1984 & 688.3 & 691.9 \\
\hline 363411116272901 & Amargosa Town Complex & 1980 & 1980 & 688.8 & 688.8 \\
\hline 363410116261101 & $\begin{array}{l}\text { Nye County Development } \\
\text { Co. }\end{array}$ & 1987 & 1987 & 691.2 & 691.2 \\
\hline 363410116240301 & PW-32 & 1966 & 1987 & 714.0 & 720.9 \\
\hline 363410116240001 & PW-33 & 1962 & 1987 & 705.9 & 723.6 \\
\hline 363407116273301 & Amargosa Valley Water & 1988 & 1988 & 701.3 & 701.3 \\
\hline 363342116335701 & PW-34 & 1958 & 1958 & 696.5 & 696.5 \\
\hline 363340116332901 & PW-35 & 1954 & 1987 & 692.4 & 696.2 \\
\hline 363342116325101 & PW-36 & 1955 & 1974 & 692.7 & 695.1 \\
\hline 363350116252101 & PW-37 & 1959 & 1962 & 698.5 & 700.4 \\
\hline 365157116271202 & USW H-1 tube 1 & 1985 & 1995 & 785.0 & 786.1 \\
\hline 365157116271203 & USW H-1 tube 2 & 1985 & 1995 & 735.7 & 736.3 \\
\hline 365157116271204 & USW H-1 tube 3 & 1985 & 1995 & 730.4 & 730.8 \\
\hline 365157116271205 & USW H-1 tube 4 & 1985 & 1995 & 730.5 & 731.0 \\
\hline 365122116275502 & USW H-5 upper & 1985 & 1995 & 775.0 & 775.7 \\
\hline 365122116275503 & USW H-5 lower & 1985 & 1995 & 775.0 & 775.9 \\
\hline 365108116262302 & UE-25 b\#1 lower & 1985 & 1995 & 728.5 & 730.3 \\
\hline 365108116262303 & UE-25 b\#1 upper & 1985 & 1995 & 730.5 & 730.8 \\
\hline 365049116285502 & USW H-6 upper & 1985 & 1995 & 775.8 & 776.2 \\
\hline 365049116285505 & USW H-6 lower & 1988 & 1995 & 775.7 & 776.1 \\
\hline 365032116265402 & USW H-4 upper & 1985 & 1995 & 730.2 & 730.5 \\
\hline 365032116265403 & USW H-4 lower & 1985 & 1995 & 730.2 & 730.8 \\
\hline 364942116280002 & USW H-3 upper & 1985 & 1995 & 731.1 & 731.9 \\
\hline 364942116280003 & USW H-3 lower & 1991 & 1996 & 747.4 & 760.3 \\
\hline 364938116252102 & UE-25 p\#1 (Lwr Intrvl) & 1985 & 1995 & 751.9 & 752.7 \\
\hline
\end{tabular}


Table A-4. Earliest Year of Measurement, Latest Year of Measurement, Minimum Water-Level Altitude, and Maximum Water-Level Altitude (DTN: GS010908312332.002) (Continued)

\begin{tabular}{|c|c|c|c|c|c|}
\hline USGS Site ID & Site Name & $\begin{array}{c}\text { Earliest Year } \\
\text { of Measurement }\end{array}$ & $\begin{array}{c}\text { Latest Year } \\
\text { of Measurement }\end{array}$ & \begin{tabular}{|c} 
Minimum \\
Water-Level \\
Altitude \\
(meters)
\end{tabular} & \begin{tabular}{|c} 
Maximum \\
Water-Level \\
Altitude \\
(meters)
\end{tabular} \\
\hline not available yet & USW SD-7 & 1995 & 1995 & 727.6 & 727.6 \\
\hline not available yet & USW SD-9 & 1994 & 1994 & 731.1 & 731.1 \\
\hline not available yet & USW SD-12 & 1995 & 1995 & 730.0 & 730.0 \\
\hline 364234116351501 & NC-EWDP-1DX, shallow & 1999 & 2000 & 786.7 & 786.8 \\
\hline 364234116351501 & NC-EWDP-1DX, deep & 1999 & 2000 & 748.7 & 748.9 \\
\hline 364233116351501 & NC-EWDP-1S, probe 1 & 1999 & 2000 & 787.1 & 787.2 \\
\hline 364233116351501 & NC-EWDP-1S, probe 2 & 1999 & 2000 & 786.7 & 786.9 \\
\hline 363939116275401 & NC-EWDP-2D & 1999 & 1999 & 706.1 & 706.2 \\
\hline 363940116275501 & NC-EWDP-2DB & 2000 & 2000 & 712.3 & 713.7 \\
\hline 364054116321401 & NC-EWDP-3D & 1999 & 2000 & 717.4 & 719.3 \\
\hline 364054116321301 & NC-EWDP-3S, probe 2 & 1999 & 2000 & 719.7 & 720.0 \\
\hline 364054116321301 & NC-EWDP-3S, probe 3 & 1999 & 2000 & 719.2 & 719.5 \\
\hline 363925116241501 & NC-EWDP-4PA & 2000 & 2000 & 717.1 & 718.7 \\
\hline 363925116241401 & NC-EWDP-4PB & 2000 & 2000 & 723.4 & 723.8 \\
\hline 364012116223401 & NC-EWDP-5SB & 2000 & 2000 & 723.4 & 723.6 \\
\hline 364332116332201 & NC-EWDP-7S & 2000 & 2000 & 829.9 & 830.2 \\
\hline 364145116334401 & NC-EWDP-9SX, probe 1 & 1999 & 2000 & 766.6 & 766.7 \\
\hline 364145116334401 & NC-EWDP-9SX, probe 2 & 1999 & 2000 & 767.2 & 767.4 \\
\hline 364145116334401 & NC-EWDP-9SX, probe 4 & 1999 & 2000 & 766.7 & 766.8 \\
\hline 364137116351001 & NC-EWDP-12PA & 2000 & 2000 & 722.8 & 723.0 \\
\hline 364138116351001 & NC-EWDP-12PB & 2000 & 2000 & 722.9 & 732.2 \\
\hline 364139116351001 & NC-EWDP-12PC & 2000 & 2000 & 720.3 & 720.8 \\
\hline 364011116294901 & NC-EWDP-15P & 2000 & 2000 & 722.4 & 722.6 \\
\hline 364015116265301 & NC-EWDP-19P & 2000 & 2000 & 707.4 & 707.7 \\
\hline 364014116265301 & NC-EWDP-19D & 2000 & 2000 & 712.6 & 712.9 \\
\hline 365301116271301 & USW WT-24 & 1999 & 1999 & 839.7 & 840.7 \\
\hline 363951116252401 & NC-Washburn-1X & 1999 & 2000 & 714.4 & 714.7 \\
\hline 364706116170601 & UE-25 J-11 & 1989 & 1995 & 732.1 & 732.4 \\
\hline 364237116365401 & BGMW-11 & 1989 & 1999 & 715.5 & 716.2 \\
\hline 363709116264601 & PW-38 & 1987 & 1987 & 704.0 & 704.0 \\
\hline 363409116233701 & PW-39 & 1962 & 1962 & 713.2 & 713.2 \\
\hline 363411116264701 & PW-40 & 1987 & 1987 & 689.5 & 689.5 \\
\hline 363428116281201 & Amargosa Water & 1987 & 1987 & 690.4 & 690.4 \\
\hline 363429116233401 & PW-41 & 1987 & 1987 & 715.7 & 715.7 \\
\hline 363511116335101 & PW-42 & 1987 & 1987 & 690.8 & 690.8 \\
\hline 365624116222901 & USW UZ-N91 & 1986 & 1996 & 1185.6 & 1191.3 \\
\hline
\end{tabular}




\section{A5. TABLE A-5: TOP OF INTERVAL, BOTTOM OF INTERVAL, AND MIDPOINT OF INTERVAL}

\section{Top of Interval/Bottom of Interval (Meters)}

Where available, the altitude of the top and bottom of screened or packed-off intervals were used. If the altitude of the screened or packed-off interval was not available, the borehole was treated as an open borehole. If the altitude of the bottom of a borehole interval was not available, the altitude of the base of the borehole was used for the bottom of the interval. Likewise, if the altitude of the top of a borehole interval was not available, the maximum water level was used for the altitude of the top of the interval. The altitudes were converted from feet to meters by the following formula:

$$
\text { Altitude }(\mathrm{ft}) \times 0.3048(\mathrm{~m} / \mathrm{ft})=\text { Altitude (meters) }
$$

The altitude was rounded to the nearest tenth of 1 meter.

\section{Midpoint of Interval (Meters)}

Most of the water levels represent a composite water-level altitude for a borehole. Composite water-level altitudes refer to water levels derived from an open interval that may encompass one or more hydrogeologic units, in which any portion of the open interval may contribute to the water level. Because the altitude at which the hydraulic head measurement applies is uncertain, the midpoint of either the water column for open (uncased) boreholes or the midpoint of a screened or packed-off interval within the borehole is identified. The altitude of the midpoint of the interval was calculated by the following formula:

$$
\text { Midpoint }=(\text { Top }+ \text { Bottom }) / 2
$$

The altitude was rounded to the nearest tenth of 1 meter.

\section{Sources}

Sources are tabulated in Table A-2.

Table A-5. Top of Interval, Bottom of Interval, and Midpoint of Interval

\begin{tabular}{|l|l|l|l|l|}
\hline \multicolumn{1}{|c|}{ USGS Site ID } & \multicolumn{1}{|c|}{ Site Name } & $\begin{array}{c}\text { Top of Interval } \\
\text { (meters) }\end{array}$ & $\begin{array}{c}\text { Bottom of Interval } \\
\text { (meters) }\end{array}$ & $\begin{array}{c}\text { Midpoint of Interval } \\
\text { (meters) }\end{array}$ \\
\hline 365629116222602 & UE-29 a\#2 & 1187.7 & 793.9 & 990.8 \\
\hline 365520116370301 & GEXA Well 4 & 1008.0 & 710.5 & 859.2 \\
\hline 365340116264601 & UE-25 WT\#6 & 1034.6 & 931.8 & 983.2 \\
\hline 365322116273501 & USW G-2 & 1020.2 & 748.0 & 884.1 \\
\hline 365239116253401 & UE-25 WT\#16 & 738.3 & 689.9 & 714.1 \\
\hline 365208116274001 & USW UZ-14 & 915.9 & 670.9 & 793.4 \\
\hline 365207116264201 & UE-25 WT\#18 & 730.8 & 713.4 & 722.1 \\
\hline 365200116272901 & USW G-1 & 754.2 & -502.9 & 125.7 \\
\hline
\end{tabular}


Table A-5. Top of Interval, Bottom of Interval, and Midpoint of Interval (Continued)

\begin{tabular}{|c|c|c|c|c|}
\hline USGS Site ID & Site Name & \begin{tabular}{|c|} 
Top of Interval \\
(meters)
\end{tabular} & $\begin{array}{c}\text { Bottom of Interval } \\
\text { (meters) }\end{array}$ & $\begin{array}{c}\text { Midpoint of Interva } \\
\text { (meters) }\end{array}$ \\
\hline 365147116185301 & UE-25 a\#3 & 748.3 & 614.5 & 681.4 \\
\hline 365140116260301 & UE-25 WT\#4 & 730.8 & 687.3 & 709.0 \\
\hline 365116116233801 & UE-25 WT\#15 & 729.2 & 668.2 & 698.7 \\
\hline 365114116270401 & USW G-4 & 730.1 & 354.2 & 542.2 \\
\hline 365105116262401 & UE-25 a\#1 & 731.0 & 436.9 & 584.0 \\
\hline 365032116243501 & UE-25 WT\#14 & 729.7 & 677.4 & 703.6 \\
\hline 365023116271801 & USW WT-2 & 730.7 & 673.4 & 702.0 \\
\hline 364947116254300 & UE-25 c\#1 & 730.3 & 216.2 & 473.2 \\
\hline 364947116254501 & UE-25 c\#3 & 730.3 & 218.3 & 474.3 \\
\hline 364947116254401 & UE-25 c\#2 & 730.2 & 376.3 & 553.2 \\
\hline 364945116235001 & UE-25 WT\#13 & 729.1 & 678.5 & 703.8 \\
\hline 364933116285701 & USW WT- 7 & 775.8 & 705.9 & 740.9 \\
\hline 364916116265601 & USW WT- 1 & 730.4 & 686.4 & 708.4 \\
\hline 364905116280101 & USW G-3 & 688.6 & -52.4 & 318.1 \\
\hline 364828116234001 & UE-25 J-13 & 707.7 & 1.8 & 354.8 \\
\hline 364825116290501 & USW WT-10 & 776.0 & 692.4 & 734.2 \\
\hline 364822116262601 & UE-25 WT\#17 & 729.7 & 681.0 & 705.4 \\
\hline 365821116343701 & USW VH-2 & 810.5 & -244.8 & 282.8 \\
\hline 364757116245801 & UE-25 WT\#3 & 729.6 & 682.0 & 705.8 \\
\hline 364732116330701 & USW VH-1 & 779.4 & 201.5 & 490.5 \\
\hline 364656116261601 & UE-25 WT\#12 & 729.5 & 675.7 & 702.6 \\
\hline 364649116280201 & USW WT-11 & 730.7 & 653.1 & 691.9 \\
\hline 364554116232400 & UE-25 J-12 & 712.6 & 606.6 & 659.6 \\
\hline 364528116232201 & UE-25 JF\#3 & 727.8 & 597.5 & 662.7 \\
\hline 364105116302601 & Cind-R-Lite Well & 729.8 & 690.6 & 710.2 \\
\hline 363907116235701 & PW-1 & 718.4 & 676.4 & 697.4 \\
\hline 363836116234001 & PW-2 & 702.8 & 648.3 & 675.6 \\
\hline 363840116235000 & PW-3 & 704.1 & 659.9 & 682.0 \\
\hline 363840116234001 & PW-4 & 705.6 & 690.4 & 698.0 \\
\hline 363840116233501 & PW-5 & 701.7 & 656.9 & 679.3 \\
\hline 363835116234001 & NDOT Well & 705.3 & 658.9 & 682.1 \\
\hline 363742116263201 & PW-6 & 706.7 & 621.8 & 664.3 \\
\hline 363830116241401 & Airport Well & 705.5 & 567.5 & 636.5 \\
\hline 363815116175901 & TW-5 & 725.1 & 652.3 & 688.7 \\
\hline 363711116263701 & PW-7 & 707.7 & 632.2 & 669.9 \\
\hline 363621116263201 & PW-8 & 704.4 & 646.2 & 675.3 \\
\hline 363549116305001 & Nye County Development Co. & 694.4 & 582.8 & 638.6 \\
\hline 363523116353701 & PW-9 & 691.9 & 655.6 & 673.8 \\
\hline 363525116325601 & PW-10 & 694.3 & 659.0 & 676.7 \\
\hline 363519116322001 & PW-11 & 694.4 & 615.1 & 654.7 \\
\hline 363540116240801 & PW-12 & 722.1 & 676.4 & 699.2 \\
\hline 363527116292501 & PW-13 & 697.8 & 637.4 & 667.6 \\
\hline
\end{tabular}


Table A-5. Top of Interval, Bottom of Interval, and Midpoint of Interval (Continued)

\begin{tabular}{|c|c|c|c|c|}
\hline USGS Site ID & Site Name & \begin{tabular}{|c|} 
Top of Interval \\
(meters)
\end{tabular} & $\begin{array}{c}\text { Bottom of Interval } \\
\text { (meters) }\end{array}$ & $\begin{array}{c}\text { Midpoint of Interval } \\
\text { (meters) }\end{array}$ \\
\hline 363521116352501 & PW-14 & 690.2 & 653.9 & 672.0 \\
\hline 363456116335501 & PW-15 & 707.4 & 649.9 & 678.6 \\
\hline 363454116314201 & PW-16 & 698.1 & 605.0 & 651.6 \\
\hline 363503116351501 & PW-17 & 691.0 & 679.1 & 685.1 \\
\hline 363503116284001 & PW-18 & 693.6 & 679.7 & 686.7 \\
\hline 363436116342301 & PW-19 & 706.9 & 664.5 & 685.7 \\
\hline 363436116333201 & $\mathrm{PW}-20$ & 699.0 & 640.1 & 669.5 \\
\hline 363434116354001 & $\mathrm{PW}-21$ & 691.3 & 650.9 & 671.1 \\
\hline 363438116324601 & $\mathrm{PW}-22$ & 695.2 & 630.3 & 662.8 \\
\hline 363442116363301 & PW-23 & 689.2 & 664.8 & 677.0 \\
\hline 363440116282401 & PW-24 & 686.4 & 642.8 & 664.6 \\
\hline 363415116275101 & PW-25 & 696.2 & 650.5 & 673.3 \\
\hline 363407116342501 & PW-26 & 691.4 & 617.5 & 654.5 \\
\hline 363407116243501 & PW-27 & 709.0 & 625.5 & 667.2 \\
\hline 363429116315901 & PW-28 & 690.4 & 637.7 & 664.0 \\
\hline 363405116321501 & PW-29 & 705.7 & 648.6 & 677.1 \\
\hline 363428116240301 & PW-30 & 717.2 & 663.1 & 690.2 \\
\hline 363428116234701 & PW-31 & 718.8 & 668.1 & 693.4 \\
\hline 363417116271801 & Nye County Land Company & 690.1 & 740.7 & 715.4 \\
\hline 363411116272901 & Amargosa Town Complex & 688.9 & 647.7 & 668.3 \\
\hline 363410116261101 & Nye County Development Co. & 691.2 & 539.5 & 615.4 \\
\hline 363410116240301 & PW-32 & 717.4 & 687.7 & 702.5 \\
\hline 363410116240001 & PW-33 & 714.8 & 662.7 & 688.7 \\
\hline 363407116273301 & Amargosa Valley Water & 701.4 & 646.5 & 673.9 \\
\hline 363342116335701 & PW-34 & 696.5 & 647.7 & 672.1 \\
\hline 363340116332901 & PW-35 & 694.2 & 635.2 & 664.7 \\
\hline 363342116325101 & PW-36 & 694.0 & 678.5 & 686.2 \\
\hline 363350116252101 & PW-37 & 699.5 & 658.4 & 678.9 \\
\hline 365157116271202 & USW H-1 tube 1 & -480.0 & -511.0 & -495.5 \\
\hline 365157116271203 & USW H-1 tube 2 & 206.0 & 180.0 & 193.0 \\
\hline 365157116271204 & USW H-1 tube 3 & 587.0 & 538.0 & 562.5 \\
\hline 365157116271205 & USW H-1 tube 4 & 731.0 & 630.0 & 680.5 \\
\hline 365122116275502 & USW H-5 upper & 775.5 & 632.9 & 704.2 \\
\hline 365122116275503 & USW H-5 lower & 632.9 & 259.9 & 446.4 \\
\hline 365108116262302 & UE-25 b\#1 lower & 1.7 & -19.3 & -8.8 \\
\hline 365108116262303 & UE-25 b\#1 upper & 730.7 & 1.7 & 366.2 \\
\hline 365049116285502 & USW H-6 upper & 776.0 & 549.8 & 662.9 \\
\hline 365049116285505 & USW H-6 lower & 549.8 & 81.8 & 315.8 \\
\hline 365032116265402 & USW H-4 upper & 730.4 & 60.5 & 395.5 \\
\hline 365032116265403 & USW H-4 lower & 60.5 & 29.5 & 45.0 \\
\hline 364942116280002 & USW H-3 upper & 731.5 & 422.2 & 576.9 \\
\hline 364942116280003 & USW H-3 lower & 422.2 & 264.2 & 343.2 \\
\hline
\end{tabular}


Table A-5. Top of Interval, Bottom of Interval, and Midpoint of Interval (Continued)

\begin{tabular}{|c|c|c|c|c|}
\hline USGS Site ID & Site Name & $\begin{array}{c}\text { Top of Interval } \\
\text { (meters) }\end{array}$ & $\begin{array}{c}\text { Bottom of Interval } \\
\text { (meters) }\end{array}$ & $\begin{array}{c}\text { Midpoint of Interval } \\
\text { (meters) }\end{array}$ \\
\hline 364938116252102 & UE-25 p\#1 (Lwr Intrvl) & -129.8 & -690.8 & -410.3 \\
\hline not available & USW SD-7 & 727.6 & 547.7 & 637.7 \\
\hline not available & USW SD-9 & 731.1 & 625.6 & 678.3 \\
\hline not available & USW SD-12 & 730.0 & 663.4 & 696.7 \\
\hline 364234116351501 & NC-EWDP-1DX, shallow & 786.8 & 384.6 & 585.7 \\
\hline 364234116351501 & NC-EWDP-1DX, deep & 145.6 & 120.6 & 133.1 \\
\hline 364233116351501 & NC-EWDP-1S, probe 1 & 754.8 & 748.8 & 751.8 \\
\hline 364233116351501 & NC-EWDP-1S, probe 2 & 739.8 & 721.8 & 730.8 \\
\hline 363939116275401 & NC-EWDP-2D & 706.1 & 308.1 & 294.3 \\
\hline 363940116275501 & NC-EWDP-2DB & -18.7 & -136 & -77 \\
\hline 364054116321401 & NC-EWDP-3D & 718.3 & 37.4 & 377.9 \\
\hline 364054116321301 & NC-EWDP-3S, probe 2 & 695.8 & 669.8 & 682.8 \\
\hline 364054116321301 & NC-EWDP-3S, probe 3 & 653.8 & 630.8 & 642.3 \\
\hline 363925116241501 & NC-EWDP-4PA & 699.0 & 675.0 & 687.0 \\
\hline 363925116241401 & NC-EWDP-4PB & 598.0 & 567.0 & 582.5 \\
\hline 364012116223401 & NC-EWDP-5SB & 724.3 & 691.3 & 707.8 \\
\hline 364332116332201 & NC-EWDP-7S & 828.4 & 824.7 & 826.6 \\
\hline 364145116334401 & NC-EWDP-9SX, probe 1 & 770.3 & 760.3 & 765.3 \\
\hline 364145116334401 & NC-EWDP-9SX, probe 2 & 754.3 & 748.3 & 751.3 \\
\hline 364145116334401 & NC-EWDP-9SX, probe 4 & 696.3 & 693.3 & 694.8 \\
\hline 364137116351001 & NC-EWDP-12PA & 675.7 & 657.7 & 666.7 \\
\hline 364138116351001 & NC-EWDP-12PB & 675.7 & 657.7 & 666.7 \\
\hline 364139116351001 & NC-EWDP-12PC & 722.7 & 704.7 & 713.7 \\
\hline 364011116294901 & NC-EWDP-15P & 725.9 & 707.9 & 716.9 \\
\hline 364015116265301 & NC-EWDP-19P & 710.2 & 679.2 & 694.7 \\
\hline 364014116265301 & NC-EWDP-19D & 713.2 & 386.2 & 549.7 \\
\hline 365301116271301 & USW WT-24 & 840.1 & 629.8 & 734.7 \\
\hline 363951116252401 & NC-Washburn-1X & 696.1 & 677.8 & 687.0 \\
\hline 364706116170601 & UE-25 J-11 & 721.2 & 653.3 & 687.2 \\
\hline 364237116365401 & BGMW-11 & 715.9 & 631.0 & 673.4 \\
\hline 363709116264601 & PW-38 & 704.1 & 775.7 & 739.9 \\
\hline 363409116233701 & PW-39 & 713.3 & 695.0 & 704.1 \\
\hline 363411116264701 & PW-40 & 689.5 & 694.1 & 691.8 \\
\hline 363428116281201 & Amargosa Water & 690.4 & 738.2 & 714.3 \\
\hline 363429116233401 & PW-41 & 715.7 & 755.3 & 735.5 \\
\hline 363511116335101 & PW-42 & 690.8 & 729.4 & 710.1 \\
\hline 365624116222901 & USW UZ-N91 & 1186.8 & 1174.4 & 1180.6 \\
\hline
\end{tabular}




\section{A6. TABLE A-6: INTERVAL DESCRIPTION AND ACCURACY OF LOCATION}

\section{Interval Description}

Where available, the interval type and description were compiled from the NWIS data files (DTN: GS991100002330.001 [DIRS 122818]) and from various Nye County datasets as identified in Table A-2.

\section{Accuracy of Location/Accuracy of Land-Surface Altitude (Meters)}

Location and land-surface altitude accuracy, where available, were compiled from the NWIS data files (DTN: GS991100002330.001 [DIRS 122818]).

\section{Sources}

Sources are tabulated in Table A-2.

Table A-6. Interval Description and Accuracy of Location

\begin{tabular}{|c|c|c|c|}
\hline USGS Site ID & Site Name & Interval Description & $\begin{array}{c}\text { Accuracy } \\
\text { of Location }\end{array}$ \\
\hline 365629116222602 & UE-29 a\#2 & Open Hole, No Screen & +/- 1 second \\
\hline 365520116370301 & GEXA Well 4 & Perforated, Porous, or Slotted Casing & $+/-1$ second \\
\hline 365340116264601 & UE-25 WT\#6 & Wire-Wound Screen & $+/-10$ seconds \\
\hline 365322116273501 & USW G-2 & Open Hole, No Screen & $+/-1$ second \\
\hline 365239116253401 & UE-25 WT\#16 & Wire-Wound Screen & $+/-1$ second \\
\hline 365208116274001 & USW UZ-14 & Fractured Rock Openings & Unknown \\
\hline 365207116264201 & UE-25 WT\#18 & Wire-Wound Screen & $+/-1$ second \\
\hline 365200116272901 & USW G-1 & Open Hole, No Screen & +/- 1 second \\
\hline 365147116185301 & UE-25 a\#3 & Open Hole, No Screen & $+/-1$ second \\
\hline 365140116260301 & UE-25 WT\#4 & Wire-Wound Screen & +/- 1 second \\
\hline 365116116233801 & UE-25 WT\#15 & Open Hole, No Screen & $+/-1$ second \\
\hline 365114116270401 & USW G-4 & Open Hole, No Screen & $+/-1$ second \\
\hline 365105116262401 & UE-25 a\#1 & Unknown & $+/-1$ second \\
\hline 365032116243501 & UE-25 WT\#14 & Wire-Wound Screen & $+/-1$ second \\
\hline 365023116271801 & USW WT-2 & Wire-Wound Screen & +/- 1 second \\
\hline 364947116254300 & UE-25 c\#1 & Composite interval - entire saturated section & $+/-1$ second \\
\hline 364947116254501 & UE-25 c\#3 & Composite interval - entire saturated section & $+/-1$ second \\
\hline 364947116254401 & UE-25 c\#2 & Upper interval - above inflatable packer & $+/-1$ second \\
\hline 364945116235001 & UE-25 WT\#13 & Open Hole, No Screen & $+/-1$ second \\
\hline 364933116285701 & USW WT- 7 & Wire-Wound Screen & +/- 1 second \\
\hline 364916116265601 & USW WT- 1 & Wire-Wound Screen & $+/-1$ second \\
\hline 364905116280101 & USW G-3 & Open Hole, No Screen & $+/-1$ second \\
\hline 364828116234001 & UE-25 J -13 & Open Hole, No Screen & $+/-1$ second \\
\hline 364825116290501 & USW WT-10 & Wire-Wound Screen & $+/-1$ second \\
\hline 364822116262601 & UE-25 WT\#17 & Wire-Wound Screen & $+/-1$ second \\
\hline 365821116343701 & USW VH-2 & Fractured Rock Openings & - \\
\hline
\end{tabular}


Table A-6. Interval Description and Accuracy of Location (Continued)

\begin{tabular}{|c|c|c|c|}
\hline USGS Site ID & Site Name & Interval Description & $\begin{array}{l}\text { Accuracy } \\
\text { of Location }\end{array}$ \\
\hline 364757116245801 & UE-25 WT\#3 & Wire-Wound Screen & $+/-1$ second \\
\hline 364732116330701 & USW VH-1 & Open Hole, No Screen & $+/-1$ second \\
\hline 364656116261601 & UE-25 WT\#12 & Wire-Wound Screen & $+/-1$ second \\
\hline 364649116280201 & USW WT-11 & Wire-Wound Screen & $+/-1$ second \\
\hline 364554116232400 & UE-25 J-12 & Perforated, Porous, or Slotted Casing & - \\
\hline 364528116232201 & UE-25 JF\#3 & Perforated, Porous, or Slotted Casing & $+/-1$ second \\
\hline 364105116302601 & Cind-R-Lite Well & Perforated, Porous, or Slotted Casing & $+/-1$ second \\
\hline 363907116235701 & $\mathrm{PW}-1$ & Perforated, Porous, or Slotted Casing & $+/-1$ second \\
\hline 363836116234001 & $\mathrm{PW}-2$ & Perforated, Porous, or Slotted Casing & $+/-1$ second \\
\hline 363840116235000 & PW-3 & Perforated, Porous, or Slotted Casing & $+/-5$ seconds \\
\hline 363840116234001 & PW-4 & Perforated, Porous, or Slotted Casing & $+/-1$ minute \\
\hline 363840116233501 & PW-5 & Perforated, Porous, or Slotted Casing & $+/-1$ second \\
\hline 363835116234001 & NDOT Well & Perforated, Porous, or Slotted Casing & $+/-1$ second \\
\hline 363742116263201 & PW-6 & Perforated, Porous, or Slotted Casing & $+/-5$ seconds \\
\hline 363830116241401 & Airport Well & Perforated, Porous, or Slotted Casing & $+/-1$ second \\
\hline 363815116175901 & TW- 5 & Open Hole, No Screen & $+/-1$ second \\
\hline 363711116263701 & PW-7 & Perforated, Porous, or Slotted Casing & $+/-5$ seconds \\
\hline 363621116263201 & $\mathrm{PW}-8$ & Perforated, Porous, or Slotted Casing & $+/-5$ seconds \\
\hline 363549116305001 & $\begin{array}{l}\text { Nye County Development } \\
\text { Co. }\end{array}$ & Perforated, Porous, or Slotted Casing & $+/-1$ second \\
\hline 363523116353701 & PW-9 & Perforated, Porous, or Slotted Casing & $+/-10$ seconds \\
\hline 363525116325601 & $\mathrm{PW}-10$ & Perforated, Porous, or Slotted Casing & $+/-5$ seconds \\
\hline 363519116322001 & PW-11 & Perforated, Porous, or Slotted Casing & $+/-5$ seconds \\
\hline 363540116240801 & $\mathrm{PW}-12$ & Perforated, Porous, or Slotted Casing & $+/-10$ seconds \\
\hline 363527116292501 & PW-13 & Unknown & $+/-5$ seconds \\
\hline 363521116352501 & PW-14 & Perforated, Porous, or Slotted Casing & $+/-5$ seconds \\
\hline 363456116335501 & PW-15 & Perforated, Porous, or Slotted Casing & $+/-1$ minute \\
\hline 363454116314201 & PW-16 & Perforated, Porous, or Slotted Casing & $+/-5$ seconds \\
\hline 363503116351501 & PW-17 & Perforated, Porous, or Slotted Casing & $+/-5$ seconds \\
\hline 363503116284001 & $\mathrm{PW}-18$ & Perforated, Porous, or Slotted Casing & $+/-5$ seconds \\
\hline 363436116342301 & PW-19 & Perforated, Porous, or Slotted Casing & $+/-5$ seconds \\
\hline 363436116333201 & PW-20 & Perforated, Porous, or Slotted Casing & $+/-5$ seconds \\
\hline 363434116354001 & $\mathrm{PW}-21$ & Perforated, Porous, or Slotted Casing & $+/-5$ seconds \\
\hline 363438116324601 & PW-22 & Perforated, Porous, or Slotted Casing & $+/-5$ seconds \\
\hline 363442116363301 & $\mathrm{PW}-23$ & Perforated, Porous, or Slotted Casing & $+/-1$ second \\
\hline 363440116282401 & PW-24 & Unknown & $+/-1$ minute \\
\hline 363415116275101 & PW-25 & Unknown & $+/-10$ seconds \\
\hline 363407116342501 & PW-26 & Perforated, Porous, or Slotted Casing & $+/-10$ seconds \\
\hline 363407116243501 & PW-27 & Unknown & $+/-1$ minute \\
\hline 363429116315901 & PW-28 & Perforated, Porous, or Slotted Casing & $+/-1$ second \\
\hline 363405116321501 & PW-29 & Perforated, Porous, or Slotted Casing & $+/-10$ seconds \\
\hline 363428116240301 & PW-30 & Perforated, Porous, or Slotted Casing & $+/-5$ seconds \\
\hline
\end{tabular}


Table A-6. Interval Description and Accuracy of Location (Continued)

\begin{tabular}{|c|c|c|c|}
\hline USGS Site ID & Site Name & Interval Description & $\begin{array}{l}\text { Accuracy } \\
\text { of Location }\end{array}$ \\
\hline 363428116234701 & PW-31 & Perforated, Porous, or Slotted Casing & $+/-5$ seconds \\
\hline 363417116271801 & $\begin{array}{l}\text { Nye County Land } \\
\text { Company }\end{array}$ & Unknown & $+/-1$ minute \\
\hline 363411116272901 & Amargosa Town Complex & Perforated, Porous, or Slotted Casing & $+/-1$ second \\
\hline 363410116261101 & $\begin{array}{l}\text { Nye County Development } \\
\text { Co. }\end{array}$ & Perforated, Porous, or Slotted Casing & $+/-5$ seconds \\
\hline 363410116240301 & PW-32 & Perforated, Porous, or Slotted Casing & $+/-5$ seconds \\
\hline 363410116240001 & PW-33 & Perforated, Porous, or Slotted Casing & $+/-5$ seconds \\
\hline 363407116273301 & Amargosa Valley Water & Perforated, Porous, or Slotted Casing & $+/-1$ second \\
\hline 363342116335701 & PW-34 & Unknown & $+/-10$ seconds \\
\hline 363340116332901 & PW-35 & Perforated, Porous, or Slotted Casing & $+/-5$ seconds \\
\hline 363342116325101 & PW-36 & Unknown & $+/-10$ seconds \\
\hline 363350116252101 & PW-37 & Perforated, Porous, or Slotted Casing & $+/-10$ seconds \\
\hline 365157116271202 & USW H-1 tube 1 & Tube 1 - deepest interval in piezometer & $+/-1$ second \\
\hline 365157116271203 & USW H-1 tube 2 & $\begin{array}{l}\text { Tube } 2 \text { - second deepest interval in } \\
\text { piezometer }\end{array}$ & $+/-1$ second \\
\hline 365157116271204 & USW H-1 tube 3 & $\begin{array}{l}\text { Tube } 3 \text { - second shallowest interval in } \\
\text { piezometer }\end{array}$ & $+/-1$ second \\
\hline 365157116271205 & USW H-1 tube 4 & Tube 4 - shallowest interval in piezometer & $+/-1$ second \\
\hline 365122116275502 & USW H-5 upper & Upper interval - above inflatable packer & $+/-1$ second \\
\hline 365122116275503 & USW H-5 lower & Lower interval - below inflatable packer & $+/-1$ second \\
\hline 365108116262302 & UE-25 b\#1 lower & Lower interval - below inflatable packer & $+/-1$ second \\
\hline 365108116262303 & UE-25 b\#1 upper & Upper interval - above inflatable packer & $+/-1$ second \\
\hline 365049116285502 & USW H-6 upper & Upper interval - above inflatable packer & $+/-1$ second \\
\hline 365049116285505 & USW H-6 lower & Lower interval - below inflatable packer & $+/-1$ second \\
\hline 365032116265402 & USW H-4 upper & Upper interval - above inflatable packer & $+/-1$ second \\
\hline 365032116265403 & USW H-4 lower & Lower interval - below inflatable packer & $+/-1$ second \\
\hline 364942116280002 & USW H-3 upper & Upper interval - above inflatable packer & $+/-1$ second \\
\hline 364942116280003 & USW H-3 lower & Lower interval - below inflatable packer & $+/-1$ second \\
\hline 364938116252102 & UE-25 p\#1 (Lwr Intrvl) & Paleozoics units monitored & $+/-1$ second \\
\hline not available yet & USW SD-7 & Fractured Rock Openings & Unknown \\
\hline not available yet & USW SD-9 & Fractured Rock Openings & Unknown \\
\hline not available yet & USW SD-12 & Fractured Rock Openings & Unknown \\
\hline 364234116351501 & NC-EWDP-1DX, shallow & Screen, above inflatable packer & Unknown \\
\hline 364234116351501 & NC-EWDP-1DX, deep & Screen, below inflatable packer & Unknown \\
\hline 364233116351501 & NC-EWDP-1S, probe 1 & Screen, between inflatable packers & Unknown \\
\hline 364233116351501 & NC-EWDP-1S, probe 2 & Screen, between inflatable packers & Unknown \\
\hline 363939116275401 & NC-EWDP-2D & Open Hole, No Screen & Unknown \\
\hline 363940116275501 & NC-EWDP-2DB & Open Hole, No Screen & Unknown \\
\hline 364054116321401 & NC-EWDP-3D & Open Hole, No Screen & Unknown \\
\hline 364054116321301 & NC-EWDP-3S, probe 2 & Screen, between inflatable packers & Unknown \\
\hline 364054116321301 & NC-EWDP-3S, probe 3 & Screen, between inflatable packers & Unknown \\
\hline 363925116241501 & NC-EWDP-4PA & Screen, above inflatable packer & Unknown \\
\hline
\end{tabular}


Table A-6. Interval Description and Accuracy of Location (Continued)

\begin{tabular}{|c|c|c|c|}
\hline USGS Site ID & Site Name & Interval Description & $\begin{array}{l}\text { Accuracy } \\
\text { of Location }\end{array}$ \\
\hline 363925116241401 & NC-EWDP-4PB & Screen, below inflatable packer & Unknown \\
\hline 364012116223401 & NC-EWDP-5SB & Screen & Unknown \\
\hline 364332116332201 & NC-EWDP-7S & Screen & Unknown \\
\hline 364145116334401 & NC-EWDP-9SX, probe 1 & Screen, between inflatable packers & Unknown \\
\hline 364145116334401 & NC-EWDP-9SX, probe 2 & Screen, between inflatable packers & Unknown \\
\hline 364145116334401 & NC-EWDP-9SX, probe 4 & Screen, below inflatable packer & Unknown \\
\hline 364137116351001 & NC-EWDP-12PA & Screen, between inflatable packers & Unknown \\
\hline 364138116351001 & NC-EWDP-12PB & Screen, between inflatable packers & Unknown \\
\hline 364139116351001 & NC-EWDP-12PC & Screen, between inflatable packers & Unknown \\
\hline 364011116294901 & NC-EWDP-15P & Screen & Unknown \\
\hline 364015116265301 & NC-EWDP-19P & Screen & Unknown \\
\hline 364014116265301 & NC-EWDP-19D & Screen, various intervals & Unknown \\
\hline 365301116271301 & USW WT-24 & Open Hole, No Screen & Unknown \\
\hline 363951116252401 & NC-Washburn-1X & Screen & Unknown \\
\hline 364706116170601 & UE-25 J-11 & Open Hole, No Screen & $+/-1$ second \\
\hline 364237116365401 & BGMW-11 & Open Hole, No Screen & +/- 1 second \\
\hline 363709116264601 & PW-38 & Unknown & $+/-1$ second \\
\hline 363409116233701 & PW-39 & Unknown & $+/-1$ minute \\
\hline 363411116264701 & PW-40 & Unknown & $+/-5$ seconds \\
\hline 363428116281201 & Amargosa Water & Unknown & $+/-5$ seconds \\
\hline 363429116233401 & $\mathrm{PW}-41$ & Unknown & $+/-5$ seconds \\
\hline 363511116335101 & PW-42 & Unknown & $+/-1$ second \\
\hline 365624116222901 & USW UZ-N91 & Open Hole, No Screen & Unknown \\
\hline
\end{tabular}




\section{A7. TABLE A-7: ACCURACY OF LAND-SURFACE ALTITUDE AND LATEST WATER-LEVEL MEASUREMENT METHOD DESCRIPTION}

\section{Accuracy of Location/Accuracy of Land-Surface Altitude (Meters)}

Location and land-surface altitude accuracy, where available, were compiled from the NWIS data files (DTN: GS991100002330.001 [DIRS 122818]).

\section{Latest Water-Level Measurement Method Description}

Typical water-level measurement method was compiled from the NWIS data files (DTN: GS991100002330.001 [DIRS 122818]) and from the Nye County datasets listed in Table 4-1.

Table A-7. Accuracy of Land-Surface Altitude and Latest Water-Level Measurement Method Description

\begin{tabular}{|l|l|l|l|}
\hline \multicolumn{1}{|c|}{ USGS Site ID } & \multicolumn{1}{|c|}{ Site Name } & $\begin{array}{c}\text { Accuracy of Land-Surface } \\
\text { Altitude (meters) }\end{array}$ & $\begin{array}{c}\text { Latest Water-Level Measurement } \\
\text { Method Description }\end{array}$ \\
\hline 365629116222602 & UE-29 a\#2 & 0.1 & Steel-tape measurement \\
\hline 365520116370301 & GEXA Well 4 & 0.1 & Electric-tape measurement \\
\hline 365340116264601 & UE-25 WT\#6 & 0.1 & Steel-tape measurement \\
\hline 365322116273501 & USW G-2 & 0.1 & Electric-tape measurement \\
\hline 365239116253401 & UE-25 WT\#16 & 0.1 & Steel-tape measurement \\
\hline 365208116274001 & USW UZ-14 & Unknown & Unknown \\
\hline 365207116264201 & UE-25 WT\#18 & 0.1 & Steel-tape measurement \\
\hline 365200116272901 & USW G-1 & 0.1 & Unknown \\
\hline 365147116185301 & UE-25 a\#3 & 0.1 & Reported, method not known \\
\hline 365140116260301 & UE-25 WT\#4 & 0.1 & Steel-tape measurement \\
\hline 365116116233801 & UE-25 WT\#15 & 0.1 & Steel-tape measurement \\
\hline 365114116270401 & USW G-4 & 0.1 & Manometer measurement \\
\hline 365105116262401 & UE-25 a\#1 & 0.1 & Calibrated electric-tape measurement \\
\hline 365032116243501 & UE-25 WT\#14 & 0.1 & Steel-tape measurement \\
\hline 365023116271801 & USW WT-2 & 0.1 & Steel-tape measurement \\
\hline 364947116254300 & UE-25 c\#1 & 0.1 & Analogue or graphic recorder \\
\hline 364947116254501 & UE-25 c\#3 & 0.1 & Steel-tape measurement \\
\hline 364947116254401 & UE-25 c\#2 & 0.1 & Reported, method not known \\
\hline 364945116235001 & UE-25 WT\#13 & 0.1 & Steel-tape measurement \\
\hline 364933116285701 & USW WT- 7 & 0.1 & Manometer measurement \\
\hline 364916116265601 & USW WT-1 & 0.1 & Electric-tape measurement \\
\hline 364905116280101 & USW G-3 & 0.1 & Unknown \\
\hline 364828116234001 & UE-25 J-13 & 0.1 & Steel-tape measurement \\
\hline 364825116290501 & USW WT-10 & 0.1 & Electric-tape measurement \\
\hline 364822116262601 & UE-25 WT\#17 & 0.1 & Steel-tape measurement \\
\hline 365821116343701 & USW VH-2 & Unknown & Unknown \\
\hline 364757116245801 & UE-25 WT\#3 & 0.1 & Steel-tape measurement \\
\hline 364732116330701 & USW VH-1 & 0.1 & Steel-tape measurement \\
\hline 364656116261601 & UE-25 WT\#12 & Steel-tape measurement \\
\hline
\end{tabular}


Water-Level Data Analysis for the Saturated Zone Site-Scale Flow and Transport Model

Table A-7. Accuracy of Land-Surface Altitude and Latest Water-Level Measurement Method Description (Continued)

\begin{tabular}{|c|c|c|c|}
\hline USGS Site ID & Site Name & $\begin{array}{c}\text { Accuracy of Land-Surface } \\
\text { Altitude (meters) }\end{array}$ & $\begin{array}{c}\text { Latest Water-Level Measurement } \\
\text { Method Description }\end{array}$ \\
\hline 364649116280201 & USW WT-11 & 0.1 & Reported, method not known \\
\hline 364554116232400 & UE-25 J-12 & 0.1 & Unknown \\
\hline 364528116232201 & UE-25 JF\#3 & 0.1 & Unknown \\
\hline 364105116302601 & Cind-R-Lite Well & 0.1 & Unknown \\
\hline 363907116235701 & $\mathrm{PW}-1$ & 1.0 & Reported, method not known \\
\hline 363836116234001 & PW-2 & 0.5 & Electric-tape measurement \\
\hline 363840116235000 & PW-3 & 3.0 & Reported, method not known \\
\hline 363840116234001 & PW-4 & 2.0 & Reported, method not known \\
\hline 363840116233501 & PW-5 & 0.5 & Reported, method not known \\
\hline 363835116234001 & NDOT Well & 0.1 & Steel-tape measurement \\
\hline 363742116263201 & PW-6 & 0.5 & Steel-tape measurement \\
\hline 363830116241401 & Airport Well & 0.1 & Calibrated electric-tape measurement \\
\hline 363815116175901 & TW- 5 & 0.1 & Electric-tape measurement \\
\hline 363711116263701 & PW-7 & 0.1 & Steel-tape measurement \\
\hline 363621116263201 & PW-8 & 0.5 & Reported, method not known \\
\hline 363549116305001 & $\begin{array}{l}\text { Nye County Development } \\
\text { Co. }\end{array}$ & 2.0 & Steel-tape measurement \\
\hline 363523116353701 & PW-9 & 0.5 & Unknown \\
\hline 363525116325601 & PW-10 & 0.5 & Electric-tape measurement \\
\hline 363519116322001 & $\mathrm{PW}-11$ & 2.0 & Steel-tape measurement \\
\hline 363540116240801 & $\mathrm{PW}-12$ & 1.0 & Unknown \\
\hline 363527116292501 & $\mathrm{PW}-13$ & 0.5 & Electric-tape measurement \\
\hline 363521116352501 & PW-14 & 0.5 & Steel-tape measurement \\
\hline 363456116335501 & $\mathrm{PW}-15$ & 0.5 & Steel-tape measurement \\
\hline 363454116314201 & PW-16 & 0.5 & Steel-tape measurement \\
\hline 363503116351501 & PW-17 & 0.5 & Steel-tape measurement \\
\hline 363503116284001 & PW-18 & 0.5 & Steel-tape measurement \\
\hline 363436116342301 & PW-19 & 2.0 & Electric-tape measurement \\
\hline 363436116333201 & PW-20 & 2.0 & Steel-tape measurement \\
\hline 363434116354001 & $\mathrm{PW}-21$ & 0.1 & Steel-tape measurement \\
\hline 363438116324601 & $\mathrm{PW}-22$ & 0.5 & Steel-tape measurement \\
\hline 363442116363301 & PW-23 & 0.5 & Reported, method not known \\
\hline 363440116282401 & PW-24 & 0.1 & Unknown \\
\hline 363415116275101 & PW-25 & 0.5 & Reported, method not known \\
\hline 363407116342501 & $\mathrm{PW}-26$ & 2.0 & Unknown \\
\hline 363407116243501 & PW-27 & 1.0 & Steel-tape measurement \\
\hline 363429116315901 & PW-28 & 0.5 & Steel-tape measurement \\
\hline 363405116321501 & PW-29 & 2.0 & Steel-tape measurement \\
\hline 363428116240301 & PW-30 & 2.0 & Steel-tape measurement \\
\hline 363428116234701 & PW-31 & 0.1 & Calibrated electric-tape measurement \\
\hline 363417116271801 & Nye County Land Company & 0.1 & Unknown \\
\hline
\end{tabular}


Water-Level Data Analysis for the Saturated Zone Site-Scale Flow and Transport Model

Table A-7. Accuracy of Land-Surface Altitude and Latest Water-Level Measurement Method Description (Continued)

\begin{tabular}{|c|c|c|c|}
\hline USGS Site ID & Site Name & $\begin{array}{c}\text { Accuracy of Land-Surface } \\
\text { Altitude (meters) }\end{array}$ & $\begin{array}{l}\text { Latest Water-Level Measurement } \\
\text { Method Description }\end{array}$ \\
\hline 363411116272901 & Amargosa Town Complex & 0.5 & Reported, method not known \\
\hline 363410116261101 & $\begin{array}{l}\text { Nye County Development } \\
\text { Co. }\end{array}$ & 0.5 & Steel-tape measurement \\
\hline 363410116240301 & PW-32 & 0.5 & Steel-tape measurement \\
\hline 363410116240001 & PW-33 & 1.0 & Steel-tape measurement \\
\hline 363407116273301 & Amargosa Valley Water & 0.5 & Reported, method not known \\
\hline 363342116335701 & PW-34 & 0.5 & Reported, method not known \\
\hline 363340116332901 & PW-35 & 2.0 & Steel-tape measurement \\
\hline 363342116325101 & $\mathrm{PW}-36$ & 2.0 & Unknown \\
\hline 363350116252101 & PW-37 & 0.5 & Steel-tape measurement \\
\hline 365157116271202 & USW H-1 tube 1 & 0.1 & Steel-tape measurement \\
\hline 365157116271203 & USW H-1 tube 2 & 0.1 & Steel-tape measurement \\
\hline 365157116271204 & USW H-1 tube 3 & 0.1 & Steel-tape measurement \\
\hline 365157116271205 & USW H-1 tube 4 & 0.1 & Steel-tape measurement \\
\hline 365122116275502 & USW H-5 upper & 0.1 & Steel-tape measurement \\
\hline 365122116275503 & USW H-5 lower & 0.1 & Steel-tape measurement \\
\hline 365108116262302 & UE-25 b\#1 lower & 0.1 & Steel-tape measurement \\
\hline 365108116262303 & UE-25 b\#1 upper & 0.1 & Steel-tape measurement \\
\hline 365049116285502 & USW H-6 upper & 0.1 & Steel-tape measurement \\
\hline 365049116285505 & USW H-6 lower & 0.1 & Unknown \\
\hline 365032116265402 & USW H-4 upper & 0.1 & Unknown \\
\hline 365032116265403 & USW H-4 lower & 0.1 & Steel-tape measurement \\
\hline 364942116280002 & USW H-3 upper & 0.1 & Steel-tape measurement \\
\hline 364942116280003 & USW H-3 lower & 0.1 & Calibrated electric-tape measurement \\
\hline 364938116252102 & UE-25 p\#1 (Lwr Intrvl) & 0.1 & Steel-tape measurement \\
\hline not available yet & USW SD-7 & Unknown & Unknown \\
\hline not available yet & USW SD-9 & Unknown & Unknown \\
\hline not available yet & USW SD-12 & Unknown & Unknown \\
\hline 364234116351501 & NC-EWDP-1DX, shallow & Unknown & Calibrated electric-tape measurement \\
\hline 364234116351501 & NC-EWDP-1DX, deep & Unknown & Calibrated electric-tape measurement \\
\hline 364233116351501 & NC-EWDP-1S, probe 1 & Unknown & Calibrated transducer \\
\hline 364233116351501 & NC-EWDP-1S, probe 2 & Unknown & Calibrated transducer \\
\hline 363939116275401 & NC-EWDP-2D & Unknown & Calibrated electric-tape measurement \\
\hline 363940116275501 & NC-EWDP-2DB & Unknown & Calibrated electric-tape measurement \\
\hline 364054116321401 & NC-EWDP-3D & Unknown & Calibrated electric-tape measurement \\
\hline 364054116321301 & NC-EWDP-3S, probe 2 & Unknown & Calibrated transducer \\
\hline 364054116321301 & NC-EWDP-3S, probe 3 & Unknown & Calibrated transducer \\
\hline 363925116241501 & NC-EWDP-4PA & Unknown & Calibrated electric-tape measurement \\
\hline 363925116241401 & NC-EWDP-4PB & Unknown & Calibrated electric-tape measurement \\
\hline 364012116223401 & NC-EWDP-5SB & Unknown & Calibrated electric-tape measurement \\
\hline 364332116332201 & NC-EWDP-7S & Unknown & Calibrated electric-tape measurement \\
\hline
\end{tabular}


Table A-7. Accuracy of Land-Surface Altitude and Latest Water-Level Measurement Method Description (Continued)

\begin{tabular}{|c|c|c|c|}
\hline USGS Site ID & Site Name & $\begin{array}{c}\text { Accuracy of Land-Surface } \\
\text { Altitude (meters) }\end{array}$ & $\begin{array}{l}\text { Latest Water-Level Measurement } \\
\text { Method Description }\end{array}$ \\
\hline 364145116334401 & NC-EWDP-9SX, probe 1 & Unknown & Calibrated transducer \\
\hline 364145116334401 & NC-EWDP-9SX, probe 2 & Unknown & Calibrated transducer \\
\hline 364145116334401 & NC-EWDP-9SX, probe 4 & Unknown & Calibrated transducer \\
\hline 364137116351001 & NC-EWDP-12PA & Unknown & Calibrated electric-tape measurement \\
\hline 364138116351001 & NC-EWDP-12PB & Unknown & Calibrated electric-tape measurement \\
\hline 364139116351001 & NC-EWDP-12PC & Unknown & Calibrated electric-tape measurement \\
\hline 364011116294901 & NC-EWDP-15P & Unknown & Calibrated electric-tape measurement \\
\hline 364015116265301 & NC-EWDP-19P & Unknown & Calibrated electric-tape measurement \\
\hline 364014116265301 & NC-EWDP-19D & Unknown & Calibrated electric-tape measurement \\
\hline 365301116271301 & USW WT-24 & Unknown & Steel-tape measurement \\
\hline 363951116252401 & NC-Washburn-1X & Unknown & Calibrated electric-tape measurement \\
\hline 364706116170601 & UE-25 J-11 & 0.1 & Calibrated electric-tape measurement \\
\hline 364237116365401 & BGMW-11 & 0.5 & Steel-tape measurement \\
\hline 363709116264601 & PW-38 & 0.5 & Steel-tape measurement \\
\hline 363409116233701 & PW-39 & 0.1 & Reported, method not known \\
\hline 363411116264701 & PW-40 & 0.1 & Steel-tape measurement \\
\hline 363428116281201 & Amargosa Water & 0.5 & Steel-tape measurement \\
\hline 363429116233401 & PW-41 & 1.0 & Steel-tape measurement \\
\hline 363511116335101 & PW-42 & 0.5 & Steel-tape measurement \\
\hline 365624116222901 & USW UZ-N91 & Unknown & Steel-tape measurement \\
\hline
\end{tabular}




\section{A8. TABLE A-8: WATER-LEVEL MEASUREMENT ACCURACY AND PERCHED IDENTIFIER}

\section{Water-Level Measurement Accuracy}

Water-level altitude accuracy, where available, was compiled from the NWIS data files (DTN: GS991100002330.001 [DIRS 122818]).

\section{Perched Identifier}

Potential perched-water levels identified during this analysis were flagged and identified as "Suspected perched” or "Assumed perched."

Table A-8. Water-Level Measurement Accuracy and Perched Identifier

\begin{tabular}{|l|l|l|l|}
\hline \multicolumn{1}{|c|}{ USGS Site ID } & \multicolumn{1}{|c|}{ Site Name } & \multicolumn{1}{|c|}{$\begin{array}{c}\text { Water-Level } \\
\text { Measurement Accuracy }\end{array}$} & \multicolumn{1}{c|}{ Perched Identifier } \\
\hline 365629116222602 & UE-29 a\#2 & Nearest 0.01 feet & Suspected perched \\
\hline 365520116370301 & GEXA Well 4 & Nearest 0.01 feet & \\
\hline 365340116264601 & UE-25 WT\#6 & Nearest 0.01 feet & Assumed perched \\
\hline 365322116273501 & USW G-2 & Unknown & Assumed perched \\
\hline 365239116253401 & UE-25 WT\#16 & Nearest 0.01 feet & \\
\hline 365208116274001 & USW UZ-14 & Unknown & \\
\hline 365207116264201 & UE-25 WT\#18 & Nearest 0.01 feet & Suspected perched \\
\hline 365200116272901 & USW G-1 & Unknown & Suspected perched \\
\hline 365147116185301 & UE-25 a\#3 & Nearest foot & Suspected perched \\
\hline 365140116260301 & UE-25 WT\#4 & Nearest 0.01 feet & \\
\hline 365116116233801 & UE-25 WT\#15 & Nearest 0.01 feet & \\
\hline 365114116270401 & USW G-4 & Nearest 0.01 feet & \\
\hline 365105116262401 & UE-25 a\#1 & Unknown & \\
\hline 365032116243501 & UE-25 WT\#14 & Nearest 0.01 feet & \\
\hline 365023116271801 & USW WT-2 & Nearest 0.01 feet & \\
\hline 364947116254300 & UE-25 C\#1 & Nearest 0.01 feet & \\
\hline 364947116254501 & UE-25 C\#3 & Nearest 0.01 feet & \\
\hline 364947116254401 & UE-25 C\#2 & Nearest foot & \\
\hline 364945116235001 & UE-25 WT\#13 & Nearest 0.01 feet & \\
\hline 364933116285701 & USW WT-7 & Nearest 0.01 feet & \\
\hline 364916116265601 & USW WT-1 & Nearest 0.1 feet & \\
\hline 364905116280101 & USW G-3 3 & Unknown & \\
\hline 364828116234001 & UE-25 J-13 & Nearest 0.01 feet & \\
\hline 364825116290501 & USW WT-10 & Nearest foot & \\
\hline 364822116262601 & UE-25 WT\#17 & Nearest 0.01 feet & \\
\hline 365821116343701 & USW VH-2 & Nnknown & \\
\hline 364757116245801 & UE-25 WT\#3 & Nearest 0.01 feet & \\
\hline 364732116330701 & USW VH-1 & Nearest 0.01 feet & \\
\hline 364656116261601 & UE-25 WT\#12 & Nearest foot & \\
\hline 364649116280201 & USW WT-11 & \\
\hline
\end{tabular}


Table A-8. Water-Level Measurement Accuracy and Perched Identifier (Continued)

\begin{tabular}{|c|c|c|c|}
\hline USGS Site ID & Site Name & $\begin{array}{c}\text { Water-Level } \\
\text { Measurement Accuracy }\end{array}$ & Perched Identifier \\
\hline 364554116232400 & UE-25 J-12 & Nearest 0.01 feet & \\
\hline 364528116232201 & UE-25 JF\#3 & Unknown & \\
\hline 364105116302601 & Cind-R-Lite Well & Nearest 0.1 feet & \\
\hline 363907116235701 & $\mathrm{PW}-1$ & Nearest foot & \\
\hline 363836116234001 & PW-2 & Nearest 0.1 feet & \\
\hline 363840116235000 & PW-3 & Nearest foot & \\
\hline 363840116234001 & PW-4 & Nearest foot & \\
\hline 363840116233501 & $P W-5$ & Nearest foot & \\
\hline 363835116234001 & NDOT Well & Nearest 0.01 feet & \\
\hline 363742116263201 & PW-6 & Nearest 0.01 feet & \\
\hline 363830116241401 & Airport Well & Nearest 0.01 feet & \\
\hline 363815116175901 & TW- 5 & Nearest 0.01 feet & \\
\hline 363711116263701 & $\mathrm{PW}-7$ & Nearest 0.01 feet & \\
\hline 363621116263201 & PW-8 & Nearest foot & \\
\hline 363549116305001 & Nye County Development Co. & Nearest 0.01 feet & \\
\hline 363523116353701 & PW-9 & Nearest 0.1 feet & \\
\hline 363525116325601 & PW-10 & Nearest 0.1 feet & \\
\hline 363519116322001 & PW-11 & Nearest 0.01 feet & \\
\hline 363540116240801 & PW-12 & Nearest 0.01 feet & \\
\hline 363527116292501 & PW-13 & Nearest 0.1 feet & \\
\hline 363521116352501 & PW-14 & Nearest 0.01 feet & \\
\hline 363456116335501 & PW-15 & Nearest 0.01 feet & \\
\hline 363454116314201 & PW-16 & Nearest 0.01 feet & \\
\hline 363503116351501 & PW-17 & Nearest 0.01 feet & \\
\hline 363503116284001 & PW-18 & Nearest 0.01 feet & \\
\hline 363436116342301 & PW-19 & Nearest 0.1 feet & \\
\hline 363436116333201 & PW-20 & Nearest 0.01 feet & \\
\hline 363434116354001 & PW-21 & Nearest 0.01 feet & \\
\hline 363438116324601 & PW-22 & Nearest 0.01 feet & \\
\hline 363442116363301 & PW-23 & Nearest foot & \\
\hline 363440116282401 & PW-24 & Nearest 0.01 feet & \\
\hline 363415116275101 & PW-25 & Nearest foot & \\
\hline 363407116342501 & PW-26 & Nearest foot & \\
\hline 363407116243501 & PW-27 & Nearest 0.01 feet & \\
\hline 363429116315901 & PW-28 & Nearest 0.01 feet & \\
\hline 363405116321501 & PW-29 & Nearest 0.01 feet & \\
\hline 363428116240301 & PW-30 & Nearest 0.01 feet & \\
\hline 363428116234701 & PW-31 & Nearest 0.01 feet & \\
\hline 363417116271801 & Nye County Land Company & Nearest 0.1 feet & \\
\hline 363411116272901 & Amargosa Town Complex & Nearest foot & \\
\hline 363410116261101 & Nye County Development Co. & Nearest 0.01 feet & \\
\hline 363410116240301 & PW-32 & Nearest 0.01 feet & \\
\hline
\end{tabular}


Table A-8. Water-Level Measurement Accuracy and Perched Identifier (Continued)

\begin{tabular}{|c|c|c|c|}
\hline USGS Site ID & Site Name & $\begin{array}{c}\text { Water-Level } \\
\text { Measurement Accuracy }\end{array}$ & Perched Identifier \\
\hline 363410116240001 & PW-33 & Nearest 0.01 feet & \\
\hline 363407116273301 & Amargosa Valley Water & Nearest foot & \\
\hline 363342116335701 & PW-34 & Nearest foot & \\
\hline 363340116332901 & PW-35 & Nearest 0.01 feet & \\
\hline 363342116325101 & PW-36 & Nearest 0.01 feet & \\
\hline 363350116252101 & PW-37 & Nearest 0.01 feet & \\
\hline 365157116271202 & USW $\mathrm{H}-1$ tube 1 & Nearest 0.01 feet & \\
\hline 365157116271203 & USW $\mathrm{H}-1$ tube 2 & Nearest 0.01 feet & \\
\hline 365157116271204 & USW $\mathrm{H}-1$ tube 3 & Nearest 0.01 feet & \\
\hline 365157116271205 & USW $\mathrm{H}-1$ tube 4 & Nearest 0.01 feet & \\
\hline 365122116275502 & USW H-5 upper & Nearest 0.01 feet & \\
\hline 365122116275503 & USW H-5 lower & Nearest 0.01 feet & \\
\hline 365108116262302 & UE-25 b\#1 lower & Nearest 0.01 feet & \\
\hline 365108116262303 & UE-25 b\#1 upper & Nearest 0.01 feet & \\
\hline 365049116285502 & USW H-6 upper & Nearest 0.01 feet & \\
\hline 365049116285505 & USW H-6 lower & Unknown & \\
\hline 365032116265402 & USW H-4 upper & Unknown & \\
\hline 365032116265403 & USW H-4 lower & Nearest 0.01 feet & \\
\hline 364942116280002 & USW H-3 upper & Nearest 0.01 feet & \\
\hline 364942116280003 & USW H-3 lower & Unknown & \\
\hline 364938116252102 & UE-25 p\#1 (Lwr Intrvl) & Nearest 0.01 feet & \\
\hline not available yet & USW SD-7 & Unknown & \\
\hline not available yet & USW SD-9 & Unknown & \\
\hline not available yet & USW SD-12 & Unknown & \\
\hline 364234116351501 & NC-EWDP-1DX, shallow & Unknown & \\
\hline 364234116351501 & NC-EWDP-1DX, deep & Unknown & \\
\hline 364233116351501 & NC-EWDP-1S, probe 1 & Unknown & \\
\hline 364233116351501 & NC-EWDP-1S, probe 2 & Unknown & \\
\hline 363939116275401 & NC-EWDP-2D & Unknown & \\
\hline 363940116275501 & NC-EWDP-2DB & Unknown & \\
\hline 364054116321401 & NC-EWDP-3D & Unknown & \\
\hline 364054116321301 & NC-EWDP-3S, probe 2 & Unknown & \\
\hline 364054116321301 & NC-EWDP-3S, probe 3 & Unknown & \\
\hline 363925116241501 & NC-EWDP-4PA & Unknown & \\
\hline 363925116241401 & NC-EWDP-4PB & Unknown & \\
\hline 364012116223401 & NC-EWDP-5SB & Unknown & \\
\hline 364332116332201 & NC-EWDP-7S & Unknown & Assumed perched \\
\hline 364145116334401 & NC-EWDP-9SX, probe 1 & Unknown & \\
\hline 364145116334401 & NC-EWDP-9SX, probe 2 & Unknown & \\
\hline 364145116334401 & NC-EWDP-9SX, probe 4 & Unknown & \\
\hline 364137116351001 & NC-EWDP-12PA & Unknown & \\
\hline 364138116351001 & NC-EWDP-12PB & Unknown & \\
\hline
\end{tabular}


Table A-8. Water-Level Measurement Accuracy and Perched Identifier (Continued)

\begin{tabular}{|l|l|l|l|}
\hline \multicolumn{1}{|c|}{ USGS Site ID } & \multicolumn{1}{|c|}{ Site Name } & \multicolumn{1}{c|}{$\begin{array}{c}\text { Water-Level } \\
\text { Measurement Accuracy }\end{array}$} & \multicolumn{1}{c|}{ Perched Identifier } \\
\hline 364139116351001 & NC-EWDP-12PC & Unknown & \\
\hline 364011116294901 & NC-EWDP-15P & Unknown & \\
\hline 364015116265301 & NC-EWDP-19P & Unknown & \\
\hline 364014116265301 & NC-EWDP-19D & Unknown & \\
\hline 365301116271301 & USW WT-24 & Nearest 0.01 feet & \\
\hline 363951116252401 & NC-Washburn-1X & Unknown & \\
\hline 364706116170601 & UE-25 J-11 & Nearest 0.01 feet & \\
\hline 364237116365401 & BGMW-11 & Nearest 0.01 feet & \\
\hline 363709116264601 & PW-38 & Nearest 0.01 feet & \\
\hline 363409116233701 & PW-39 & Nearest foot & \\
\hline 363411116264701 & PW-40 & Nearest 0.01 feet & \\
\hline 363428116281201 & Amargosa Water & Nearest 0.01 feet & Suspected perched \\
\hline 363429116233401 & PW-41 & Nearest 0.01 feet & \\
\hline 363511116335101 & PW-42 & Nearest 0.01 feet & Nearest 0.01 feet \\
\hline 365624116222901 & USW UZ-N91 & & \\
\hline
\end{tabular}

Table A-9. List of Private Wells and the Corresponding Symbol Name

\begin{tabular}{|c|c|c|c|}
\hline Private Well Name & Private Well ID \# & Private Well Name & Private Well ID \# \\
\hline Ben Bossingham & PW 1 & Davidson Well & PW 14 \\
\hline Fred Cobb & PW 2 & Eugene J. Mankinen & PW 15 \\
\hline Bob Whellock & PW 3 & Donald O. Heath & PW 16 \\
\hline Louise Pereidra & PW 4 & Elvis Kelley & PW 17 \\
\hline Joe Richards & PW 5 & Manuel Rodela & PW 18 \\
\hline James H. Shaw & PW 6 & Charles C. DeFir Jr. & PW 19 \\
\hline Richard Washburn & PW 7 & William R. Monroe & PW 20 \\
\hline Richard Washburn & PW 8 & DeFir Well & PW 21 \\
\hline Fred Woolridge & PW 9 & Edwin H. Mankinen & PW 22 \\
\hline Fred J. Keefe & PW 10 & Bill Strickland & PW 23 \\
\hline Leslie Nickels & PW 11 & M. Meese & PW 24 \\
\hline L. Mason & PW 12 & Theo E. Selbach & PW 25 \\
\hline Unknown & PW 13 & C. L. Caldwell & PW 26 \\
\hline Leonard Siegel & PW 27 & Lewis N. Dansby & PW 35 \\
\hline James K. Pierce & PW 28 & Edwin H. Mankinen & PW 36 \\
\hline James K. Pierce & PW 29 & Willard Johns & PW 37 \\
\hline Cooks West Well & PW 30 & Richard Washburn & PW 38 \\
\hline Cooks East Well & PW 31 & L. Cook & PW 39 \\
\hline Lewis C. Cook & PW 32 & Unknown & PW 40 \\
\hline Lewis C. Cook & PW 33 & Lewis C. Cook & PW 41 \\
\hline Earl N. Selbach & PW 34 & Unknown & PW 42 \\
\hline
\end{tabular}




\section{APPENDIX B}

\section{QUALIFICATION OF WATER-LEVEL DATA}


This appendix contains the qualification of two water-level data sets. Qualification of Water-level Data Collected in Well UE-25 p\#1 is found immediately below and Qualification of Water-level Data Collected in Well UE-25 J-13 is found beginning on page B-6. The data from Well UE-25 p\#1 is considered unqualified project data per AP-SIII.9Q and is qualified within this work product per AP-SIII.2Q to provide a desired level of confidence that the data are suitable for the intended use of determining vertical gradients. The data from Well UE-25 J-13 was collected in 1963 and is considered data from an outside source per AP-SIII.9Q, Section 5.2.1 l. The decision was made to also qualify this data per AP-SIII.2Q to provide a consistent treatment of the two data sets.

\section{QUALIFICATION OF WATER-LEVEL DATA COLLECTED IN WELL UE-25 P\#1. DTN: GS920408312314.009 Table S97274_001}

\section{Data Set for Qualification}

The qualification of this data set is intended to provide a desired level of confidence that the data is suitable for the intended use of determining the vertical hydraulic head difference between the Paleozoic carbonate aquifer and the overlying Tertiary age aquifers at the UE-25 p\#1 location. The Qualification Plan for this data is presented in Appendix C.

The data set for qualification consists of 23 rows of data obtained from DTN: GS920408312314.009 Table S97274_001 and are shown in Table B-1. The data are water-level measurements collected during packer testing of Borehole UE-25 p\#1. The static water level in each packed off interval of the borehole was recorded during the packer testing as part of the method to determine hydraulic properties of the different intervals in the borehole.

The tested intervals and corresponding water-level data are separated into two groups, Tertiary Section and Paleozoic Section, representing the primary hydrogeologic unit of the tested interval. The data have been previously published by Craig and Robinson (1984 [DIRS 101040], Table 2). The data in the original field notebooks can be viewed in record MOL.20000301.0894, pp. 95 to 100 and 298 to 314 (USGS n.d [DIRS 171099]).

The water-level data are to be used in this water-level analysis report (Table 6-4) to document the vertical hydraulic head difference between the carbonate aquifer and the overlying volcanic aquifers.

Table B-1. Water-Level Data from DTN: GS920408312314.009 Table S97274_001

\begin{tabular}{|l|l|l|l|l|l|}
\hline ROW \# & $\begin{array}{c}\text { Water Table } \\
\text { Altitude } \\
\text { (masl) }\end{array}$ & \multicolumn{1}{|c|}{ Location } & $\begin{array}{c}\text { Depth Interval } \\
\text { (meters) }\end{array}$ & $\begin{array}{c}\text { Depth to Water } \\
\text { (meters) }\end{array}$ & \multicolumn{1}{c|}{ Comments } \\
\hline 1 & 729.9 & UE-25 p\#1 & Static-500 & 383.9 & Tertiary section \\
\hline 2 & 730.4 & UE-25 p\#1 & $500-550$ & 383.5 & Tertiary section \\
\hline 3 & 729.9 & UE-25 p\#1 & $550-600$ & 383.9 & Tertiary section \\
\hline 4 & 730.6 & UE-25 p\#1 & $739-789$ & 383.3 & Tertiary section \\
\hline 5 & 730.8 & UE-25 p\#1 & $764-834$ & 383.1 & Tertiary section \\
\hline
\end{tabular}


Table B-1. Water-Level Data From DTN: GS920408312314.009 Table S97274_001 (Continued)

\begin{tabular}{|c|c|c|c|c|c|}
\hline ROW \# & $\begin{array}{c}\text { Water Table } \\
\text { Altitude } \\
\text { (masl) }\end{array}$ & Location & $\begin{array}{c}\text { Depth Interval } \\
\text { (meters) }\end{array}$ & $\begin{array}{c}\text { Depth to Water } \\
\text { (meters) }\end{array}$ & Comments \\
\hline 6 & 732.7 & UE-25 p\#1 & $834-904$ & 381.1 & Tertiary section \\
\hline 7 & 731.7 & UE-25 p\#1 & 904-974 & 382.2 & Tertiary section \\
\hline 8 & 733.0 & UE-25 p\#1 & 974-1044 & 380.9 & Tertiary section \\
\hline 9 & 734.5 & UE-25 p\#1 & $1044-1114$ & 379.4 & Tertiary section \\
\hline 10 & 752.2 & UE-25 p\#1 & $1110-1180$ & 361.7 & Tertiary section \\
\hline 11 & 751.9 & UE-25 p\#1 & $1297-1308$ & 362.0 & Paleozoic section \\
\hline 12 & 751.6 & UE-25 p\#1 & $1297-1338$ & 362.3 & Paleozoic section \\
\hline 13 & 751.6 & UE-25 p\#1 & $1341-1381$ & 362.3 & Paleozoic section \\
\hline 14 & 751.5 & UE-25 p\#1 & $1381-1420$ & 362.4 & Paleozoic section \\
\hline 15 & 751.4 & UE-25 p\#1 & $1423-1463$ & 362.5 & Paleozoic section \\
\hline 16 & 751.5 & UE-25 p\#1 & 1463-1509 & 362.4 & Paleozoic section \\
\hline 17 & 751.3 & UE-25 p\#1 & 1509-1554 & 362.6 & Paleozoic section \\
\hline 18 & 751.4 & UE-25 p\#1 & $1554-1585$ & 362.5 & Paleozoic section \\
\hline 19 & 751.2 & UE-25 p\#1 & $1597-1643$ & 362.7 & Paleozoic section \\
\hline 20 & 750.9 & UE-25 p\#1 & $1643-1689$ & 363.0 & Paleozoic section \\
\hline 21 & 751.0 & UE-25 p\#1 & 1689-1734 & 362.9 & Paleozoic section \\
\hline 22 & 750.8 & UE-25 p\#1 & $1734-1780$ & 363.1 & Paleozoic section \\
\hline 23 & 750.9 & UE-25 p\#1 & 1780-1805 & 363.0 & Paleozoic section \\
\hline
\end{tabular}

\section{Method of Qualification}

The method chosen to qualify this data is the corroborating data method. This method is appropriate for this data set because corroborating data are available for comparison and inferences drawn to corroborate the unqualified data can be clearly identified, justified, and documented.

The water-level data from Well UE-25 p\#1 to be qualified (Table B-1) can be grouped into the Tertiary or Paleozoic section according to the rock unit of the tested interval. Corroborating data from the Tertiary section come from water-level measurements in six wells located from $600 \mathrm{~m}$ to 2,500 $\mathrm{m}$ away from Well UE-25 p\#1. The six wells are UE-25c\#1, UE-25c\#2, UE-25c\#3, UE-25 WT\#13, UE-25 WT\#14, and USW WT-1. The water-level data for these six wells is qualified and is documented in Table A-1 of this report. The water-level data from these six wells are appropriate for corroboration because they are located near Well UE-25 p\#1 in the low-gradient region where water levels vary only a small amount spatially. The corroborating data are averages of measured water levels for periods of time from 1 to 10 years. The long-term average water levels and the similarity of water levels in the low-gradient region make the measured water levels in these six wells appropriate for corroborating the water levels in the Tertiary section of UE-25 p\#1.

Corroborating data for the Paleozoic section come from water-level measurements in Well UE-25 p\#1 from the time period of 1985 to 1995 as documented in this report (Tables A-1 
and A-4). These data are qualified and were obtained after Well UE-25 p\#1 was completed and open only to the Paleozoic section. These corroborating data are appropriate because they were measured in the same well as the unqualified data, are qualified, and represent a long-term record of measurements. There is additional data available for corroboration from the U.S. Geological Survey NWIS Web database. This database contains data from October 1983 through February 2004. The data are unqualified, but are sufficient for corroborating purposes.

\section{Evaluation Criteria}

The water level as measured in the Tertiary section of Well UE-25 p\#1 is considered corroborated if it falls within $1.5 \mathrm{~m}$ of the average water levels from the six corroborating wells. The 1.5-m criterion is the maximum water-level uncertainty as determined from the uncertainty analyses in Section 6.5. As seen in Figure 6-1, the potentiometric surface in this region has a low-hydraulic gradient, and there is a small range in average water levels among the six corroborating wells. Thus, this criterion provides a high degree of confidence that the data to be qualified are acceptable for estimating the vertical hydraulic head difference.

The water level in the Paleozoic section is corroborated by other water-level measurements in the same well from the time period of 1983 to 2004. Again, the criterion for corroboration is that the unqualified data fall within $1.5 \mathrm{~m}$ of the corroborating water levels from the deep completion in well UE-25 p\#1. The 1.5-m criterion is the maximum water-level uncertainty as determined from the uncertainty analyses in Section 6.5.

\section{Evaluation of the Technical Correctness of the Data}

In accordance with procedure AP-SIII.2Q (Qualification of Unqualified Data) the following steps are being taken to evaluate the technical correctness of this data set. First, an initial evaluation of the data quality and correctness will be presented by comparing the methods used to plan, collect, and analyze the data against accepted scientific practice. Second, the data corroboration approach will be used to compare the unqualified data to equivalent corroborating qualified data.

The field operations associated with the drilling and testing of Borehole UE-25 p\#1 are documented in the data record (USGS [n.d] [DIRS 171099]). The record contains 3,080 pages of information including field notebooks, time logs, and copies of the data collection forms and the hard copy of the data collected. The data of interest associated with packer testing are presented in Field Notebooks (USGS [n.d] [DIRS 171099], pp. 95 to 101 for the Tertiary section and pp. 293 to 315 for the Paleozoic section). The notebooks document the names of scientists and technicians performing the work, the date and time activities occurred, the type of data collected, method used to collect water-level data (transducer or float switch), and the data values themselves. Testing methods at the time of data collection were standardized as noted by Blankennagel (1967 [DIRS 103092]). Updates to equipment have occurred since 1967, but the Blankennagel reference demonstrates that the USGS has followed established procedures for many years. The analysis of the data is documented in the field notebooks and follows a standard approach of collecting a depth to water measurement from a reference point, then correcting that measurement for the distance of the measuring point above land surface. In 
summary, the water-level data were collected by a well established and known agency (the USGS) using established USGS methods and were carefully documented in field notebooks.

The water-level data collected by the U.S. Geological Survey during packer testing of Well UE-25 p\#1 are deemed adequate for implementation of the corroborating data method of qualification.

Tertiary Section: Ten water-level measurement intervals are presented from the static water position to a depth of $1,114 \mathrm{~m}$. The only data point used in this report is the water level from the interval of static $-500 \mathrm{~m}$ because this value is expected to best represent the water table. From Table B-1, the water-level altitude for the interval of static-500 m is 729.9 masl.

The six wells nearest to UE-25 p\#1 have been identified from Figure 1-2. These six wells are: UE-25 c\#1, UE-25 c\#2, UE-25 c\#3, UE-25 WT\#13, UE-25 WT\#14, and USW WT-1. Table B-2 contains the well name, depth interval, and water-level elevation for comparison to UE-25 p\#1. The data for corroboration are taken from Tables A-1 and A-5 of this document. The interval identified in Table B-2 is modified from Table A-5 in two ways. First, the term static is used if the top of the interval is the measured water level. Second, the bottom of the interval is converted from elevation to depth by subtracting the interval-bottom elevation (Table A-5) from the land surface elevation in Table A-1.

The corroborating wells are located from approximately $600 \mathrm{~m}$ to 2,500 $\mathrm{m}$ away from UE-25 p\#1. The land surface elevation of the wells are all similar, with the elevation of UE-25 p\#1 between the range of elevations of the corroborating wells. The intervals all begin at the static water table and extend downward. The lower depth of the corroborating well intervals ranges from $354 \mathrm{~m}$ to $914 \mathrm{~m}$. In general, the completion intervals and land surface elevations of the UE-25 p\#1 data and the corroborating data are similar. The measured water level in UE-25 \#1 of 729.9 falls between the average water levels in the corroborating wells. In addition, the corroborating wells with higher land surface elevation also have a higher water-level elevation. The criterion of qualification of the water level in UE-25 p\#1 is met because the water level is within $1.5 \mathrm{~m}$ of the corroborating data. Thus, there is consistency among all the wells that support the qualification of the shallow interval water level measured in Well UE-25 p\#1.

Table B-2. Corroborating Data for UE-25 p\#1, Tertiary Section

\begin{tabular}{|c|c|c|c|}
\hline Well Name & $\begin{array}{c}\text { Interval (depth below land } \\
\text { surface (meters)) }\end{array}$ & $\begin{array}{c}\text { Land Surface } \\
\text { Elevation } \\
\text { (meters) }\end{array}$ & $\begin{array}{c}\text { Water Level } \\
\text { (meters) }\end{array}$ \\
\hline UE-25 $\mathrm{p \# 1}$ & Static-500 & 1113.9 & 729.9 \\
\hline UE-25 c\#1 & Static-914 & 1130.6 & 730.2 \\
\hline UE-25 c\#2 & Static-756 & 1132.2 & 730.1 \\
\hline UE-25 c\#3 & Static-914 & 1132.4 & 730.2 \\
\hline UE-25 WT\#13 & Static-354 & 1032.5 & 729.1 \\
\hline UE-25 WT\#14 & Static-399 & 1076.4 & 729.7 \\
\hline USW WT-1 & Static-515 & 1201.4 & 730.4 \\
\hline
\end{tabular}


Paleozoic Section: Thirteen water-level measurements in the Paleozoic section are presented in Table B-1. Of those 13 measurements, the first two represent nearly the same interval. For the analysis of the water level in the Paleozoic section, the last 12 measurements are averaged and produce a value of $751.26 \mathrm{~m}$, with a range of values from $750.8 \mathrm{~m}$ to $751.6 \mathrm{~m}$. From Tables A-1 and A-4, the average water level in the carbonate completion in Well UE-25 p\#1 collected over the time period of 1985 to 1995 is $752.4 \mathrm{~m}$ with a range of values from 751.9 to 752.7 . The difference in the average water levels is $1.14 \mathrm{~m}$. Additional water-level information is available from the U.S. Geologic Survey NWIS Web Site (http://waterdata.usgs.gov/nwis) for the carbonate completion of Well UE-25 p\#1 (Site ID 364938116252102). Water levels in Well UE-25 p\#1 in late 1983, throughout 1984, and into early 1985 range from $749.47 \mathrm{~m}$ to $752.1 \mathrm{~m}$, similar to the values in Table B-1. Water levels appear to have risen since 1985, thus the difference of $1.14 \mathrm{~m}$ between the data in Tables B-1 and A-4 may be due, in part, to natural temporal variability in water levels. Nonetheless, the water level measured in the Paleozoic section during packer testing in 1983 (as presented in Table B-1) may be in error by $1.14 \mathrm{~m}$ based on average values. Even if the error is $1.14 \mathrm{~m}$, the water levels for the Paleozoic section in Table B-2 are adequate for the intended purpose in this document because the difference is less than the 1.5 criterion defined for qualification.

\section{Evaluation of Results, Conclusion for Qualification, and Limitations}

The water-level difference between the Paleozoic section (carbonate aquifer) and the water table in the Tertiary section (volcanic aquifers) is given as $21.4 \mathrm{~m}$ in Table 6-4. Even if the values in Table B-2 are in error by $3 \mathrm{~m}$ (two times the criterion), it would produce only a 15-percent error in the calculated vertical hydraulic head difference. In fact, the error is less than $3 \mathrm{~m}$ based on the similarity of corroborating data in the Tertiary section and $1.14 \mathrm{~m}$ difference in the Paleozoic section. It is expected that the potential error in the UE-25 p\#1 data produces between a 5 -percent and 10-percent error in the calculated vertical water-level difference.

The conclusion of this qualification process is the water levels in Table B-1 for Well UE-25 p\#1 are adequate for the intended use in this document and should be considered qualified.

The data as qualified are considered to be accurate with at most a 10-percent error. Future users of the data should be cognizant of this potential error.

The qualification of this data is for use in this document only. 


\section{QUALIFICATION OF WATER LEVEL DATA COLLECTED IN WELL UE-25 J-13. DTN: GS930408312132.007 TABLE S97276_013}

\section{Data Set for Qualification}

The qualification of this data set is intended to provide a desired level of confidence that the data is suitable for the intended use of determining the vertical hydraulic head difference within the Tertiary age aquifers at the UE-25 J-13 location. The Qualification Plan for this data is presented in Appendix C.

The data set for qualification consists of 13 rows of data obtained from DTN: GS930408312132.007 Table S97276_013 [DIRS 129625] and are shown in Table B-3. The data are water-level measurements collected during packer testing of Borehole UE-25 J-13. The static water level in each packed off interval of the borehole was recorded during the packer testing as part of the method to determine hydraulic properties of the different intervals in the borehole.

The data in the above-referenced DTN and table have been previously published by Thordarson (1983 [DIRS 101057]), in Geohydrologic Data and Test Results from Well J-13, Nevada Test Site, Nye County, Nevada. U.S. Geological Survey Water-Resources Investigation Report 83-4171. ACC: NNA.19870518.0071.

The water-level data are to be used in this water-level analysis report (Table 6-4) to document the vertical hydraulic head difference within the volcanic aquifers at the UE-25 J-13 location.

Table B-3. Water-Level Data from DTN: GS930408312132.007 Table S97276_013

\begin{tabular}{|l|l|l|l|l|l|}
\hline ROW \# & $\begin{array}{c}\text { Depth to } \\
\text { Water } \\
\text { (meters) }\end{array}$ & \multicolumn{1}{|c|}{ Location } & $\begin{array}{c}\text { Depth Interval } \\
\text { (meters) }\end{array}$ & Tithostratigraphy & \multicolumn{1}{|c|}{ Test } \\
\hline 1 & 282.2 & UE-25 J-13 & $282.2-334.1$ & $\begin{array}{l}\text { Topopah Spring } \\
\text { Member }\end{array}$ & - \\
\hline 2 & 282.5 & UE-25 J-13 & $282.5-451.1$ & $\begin{array}{l}\text { Topopah Spring } \\
\text { Member }\end{array}$ & Pumping 1 \\
\hline 3 & 282.7 & UE-25 J-13 & $282.7-451.1$ & $\begin{array}{l}\text { Topopah Spring } \\
\text { Member }\end{array}$ & Pumping 2 \\
\hline 4 & 282.5 & UE-25 J-13 & $471.2-502.0$ & $\begin{array}{l}\text { Tuffaceous beds } \\
\text { of Calico Hills }\end{array}$ & Injection 19 \\
\hline 5 & 282.3 & UE-25 J-13 & $471.2-502.0$ & $\begin{array}{l}\text { Tuffaceous beds } \\
\text { of Calico Hills }\end{array}$ & Swabbing 19 \\
\hline 6 & 282.4 & UE-25 J-13 & $501.1-562.1$ & $\begin{array}{l}\text { Tuffaceous beds } \\
\text { of Calico Hills and } \\
\text { Prow Pass } \\
\text { Member }\end{array}$ & Injection 16 \\
\hline 7 & 282.2 & UE-25 J-13 & $501.1-562.1$ & $\begin{array}{l}\text { Tuffaceous beds } \\
\text { of Calico Hills and } \\
\text { Prow Pass } \\
\text { Member }\end{array}$ & Swabbing 18 \\
\hline
\end{tabular}


Table B-3. Water-Level Data from DTN: GS930408312132.007 Table S97276_013 (Continued)

\begin{tabular}{|c|c|c|c|c|c|}
\hline ROW \# & $\begin{array}{c}\text { Depth to } \\
\text { Water } \\
\text { (meters) }\end{array}$ & Location & $\begin{array}{c}\text { Depth Interval } \\
\text { (meters) }\end{array}$ & Lithostratigraphy & Test \\
\hline 8 & 282.0 & UE-25 J-13 & $471.2-612.6$ & $\begin{array}{l}\text { Tuffaceous beds } \\
\text { of Calico Hills, } \\
\text { Prow Pass } \\
\text { Member, and } \\
\text { tuffaceous } \\
\text { sandstone }\end{array}$ & Swabbing 2 \\
\hline 9 & 282.4 & UE-25 J-13 & $471.2-612.6$ & $\begin{array}{l}\text { Tuffaceous beds } \\
\text { of Calico Hills, } \\
\text { Prow Pass } \\
\text { Member, and } \\
\text { tuffaceous } \\
\text { sandstone }\end{array}$ & Swabbing 3 \\
\hline 10 & 282.1 & UE-25 J-13 & $471.2-661.4$ & $\begin{array}{l}\text { Tuffaceous beds } \\
\text { of Calico Hills, } \\
\text { Prow Pass } \\
\text { Member, } \\
\text { tuffaceous } \\
\text { sandstone, and } \\
\text { Bullfrog Member }\end{array}$ & Swabbing 6 \\
\hline 11 & 282.4 & UE-25 J-13 & $584.6-645.6$ & $\begin{array}{l}\text { Prow Pass } \\
\text { Member, } \\
\text { tuffaceous } \\
\text { sandstone, and } \\
\text { Bullfrog Member }\end{array}$ & Injection 15 \\
\hline 12 & $283.6+/-2^{*}$ & UE-25 J-13 & $772.7-803.1$ & Tram Unit & Swabbing 11 \\
\hline 13 & 283.3 & UE-25 J-13 & 819.9-1063.1 & $\begin{array}{l}\text { Tram Unit, bedded } \\
\text { tuff, and Tuff of } \\
\text { Lithic Ridge }\end{array}$ & Swabbing 20 \\
\hline
\end{tabular}

* Indicates nearly recovered to static water level after 270 minutes.

\section{Method of Qualification}

The method chosen to qualify this data is the corroborating data method. This method is appropriate for this data set because corroborating data are available for comparison and inferences drawn to corroborate the unqualified data can be clearly identified, justified, and documented.

The water-level data from Well UE-25 J-13 to be qualified (Table B-3) represent different units within the Tertiary volcanic sequence at the J-13 location. Corroborating data from the Tertiary section come from water-level measurements in Well UE-25 J-13 from the time period of 1986 to 1995 as documented in this report (Tables A-1 and A-4). These data are qualified and were obtained after Well UE-25 J-13 was completed. These corroborating data are appropriate because they were measured in the same well as the unqualified data, are qualified, and represent a long-term record of measurements. There is additional data available for corroboration from the USGS NWIS Web database. This database contains data from December 1962 through June 2004. These data are unqualified, but are sufficient for corroborating purposes. 


\section{Evaluation Criteria}

The water level as measured in the Tertiary section of Well UE-25 J-13 is considered corroborated if it falls within $1.5 \mathrm{~m}$ of other water-level measurements in the same well from the time period of 1962 to 2004. The criterion for corroboration is that the unqualified data fall within $1.5 \mathrm{~m}$ of the corroborating water levels from completed Well UE-25 J-13. The 1.5-m criterion is the maximum water-level uncertainty as determined from the uncertainty analyses in Section 6.5.

\section{Evaluation of the Technical Correctness of the Data}

In accordance with procedure AP-SIII.2Q (Qualification of Unqualified Data) the following steps are being taken to evaluate the technical correctness of this data set. First, an initial evaluation of the data quality and correctness will be presented by comparing the methods used to plan, collect, and analyze the data against accepted scientific practice. Second, the data corroboration approach will be used to compare the unqualified data to equivalent corroborating qualified data.

The data to be qualified are the static water levels measured during hydraulic testing and presented as the depth to water in DTN: GS930408312132.007 [DIRS 129625], Table S97276_013. The data were previously published by Thordarson (1983 [DIRS 101057], Table 10, p. 21). The data were obtained in 1962 and 1963 during the construction of Well UE-25 J-13. Established procedures for making these water-level measurements using a device called the "Iron Horse" are documented by Weir and Nelson (1976 [DIRS 170983]). Personnel performing this work had been involved with hydrology studies in Southern Nevada for a number of years. Therefore, the reliability of the data source and qualifications of personnel working for the USGS collecting the data are sufficient. The period of record for this well as provided by the USGS in the NWIS Groundwater Database spans the time frame of December 30, 1962, to June 29, 2004, and contains 253 water-level measurements. The range of hydraulic head values over this time period ranges from $728.0 \mathrm{~m}$ to $729.0 \mathrm{~m}$ above sea level (using the USGS depth to water values and land surface datum of $1011.3 \mathrm{~m}$ amsl - Table A-1). Savard (2001 [DIRS 165604], p. 73) presents analysis of water-level data and showed that average water-level elevation in the J-13 well over the period of 1986 to 1999 was about $728.5 \mathrm{~m}$. The water-level data reported in Table A-4 of this report cover the time period of 1986 to 1995 and range from $728.3 \mathrm{~m}$ to $728.7 \mathrm{~m}$. The data from DTN: GS930408312132.007, [DIRS 129625] Table S97276_013 fall within the range of values from $727.7 \mathrm{~m}$ to $729.3 \mathrm{~m}$ using the provided depth to water and land surface elevation from Table A-4 of $1011.3 \mathrm{~m}$ amsl. Based on the similarity of water levels measured in Well UE-25 J-13 during the packer testing and over the time period of 1962 to 2004, the data from DTN: GS930408312132.007, [DIRS 129625], Table S97276_013 are considered acceptable for use in this report.

\section{Evaluation of Results, Conclusion for Qualification, and Limitations}

The water levels measured in Well UE-25 J-13 have been shown to be very stable, varying over only $1 \mathrm{~m}$ from 1962 to 2004. The water levels measured during hydraulic testing in Well UE-25 $\mathrm{J}-13$ fall from $0.3 \mathrm{~m}$ below to $0.3 \mathrm{~m}$ above the range of values reported for this well. The 
criterion for qualification of less than a $1.5 \mathrm{~m}$ difference between the unqualified data and the corroborating data has been met.

The conclusion of this qualification process is the water levels in Table B-3 for Well UE-25 J-13 are adequate for the intended use in this document and should be considered qualified.

The data as qualified are considered to be accurate within about $1 \mathrm{~m}$. Future users of the data should be cognizant of this potential error.

The qualification of this data is for use in this document only. 


\section{INTENTIONALLY LEFT BLANK}




\section{APPENDIX C}

\section{DATA QUALIFICATION PLANS}


This appendix contains the Data Qualification Plans for the qualification of water-level data from Wells UE-25 p\#1 and UE-25 J-13. The plans were modified as noted in the hand written comments. The modifications were added to provide missing information or to clarify the existing text. 


\begin{tabular}{|c|c|c|}
\hline \multirow{2}{*}{ BSC } & \multirow{2}{*}{ DATA QUALIFICATION PLAN } & QA: QA \\
\hline & & Page 1 of 1 \\
\hline \multicolumn{3}{|c|}{ Section I. Organizational Information } \\
\hline \multicolumn{3}{|l|}{ Qualification Title } \\
\hline \multicolumn{3}{|c|}{ UE25P\#1 Water Level Data Collected During Packer Testing } \\
\hline \multicolumn{3}{|c|}{ Requesting Organization } \\
\hline \multicolumn{3}{|c|}{ RIT Natural Systems Team } \\
\hline \multicolumn{3}{|c|}{ Section II. Process Planning Requirements } \\
\hline \multicolumn{3}{|c|}{$\begin{array}{l}\text { 1. List of Unqualified Data to be Evaluated } \\
\text { Data from DTN: GS920408312314.009, T }\end{array}$} \\
\hline \multirow{2}{*}{\multicolumn{3}{|c|}{ 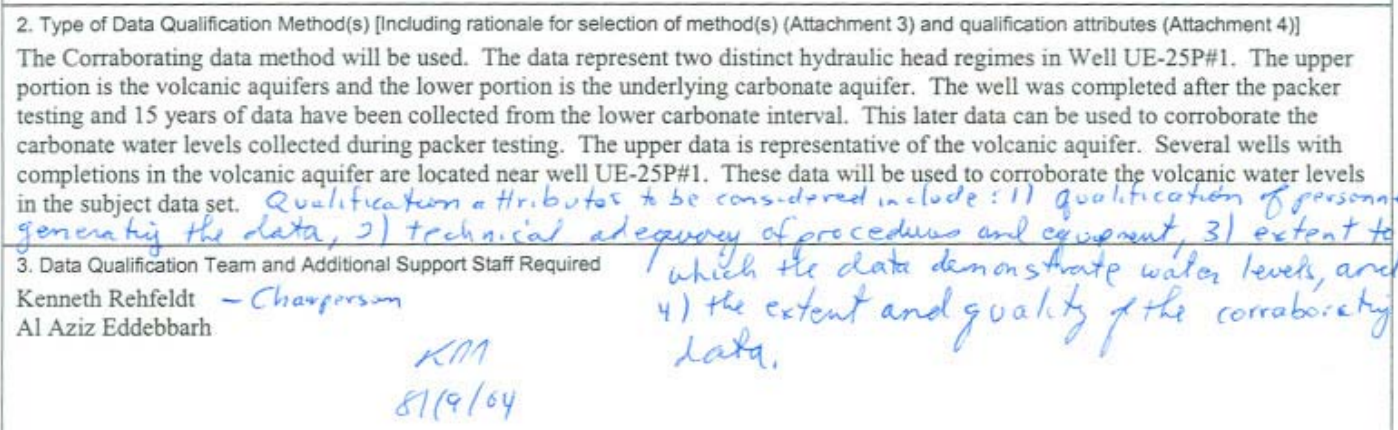 }} \\
\hline & & \\
\hline \multirow{2}{*}{\multicolumn{3}{|c|}{$\begin{array}{l}\text { 4. Data Evaluation Criteria } \\
\text { The data will be evaluated to show that water levels from UE-25P\#1 during packer testing are consistent with the corroborating data. } \\
\text { In this regard consistency is defined to mean that data from UE-25P\#1 packer testing fall within the range of values of the } \\
\text { corroborating data. The comporisen need, to accomat for the poten that error in } \\
\text { weter level measurements of } 1.5 m \text { as presented in section } 6.5 \text { the } \\
\text { docoment. }\end{array}$}} \\
\hline & & \\
\hline \multicolumn{3}{|c|}{ 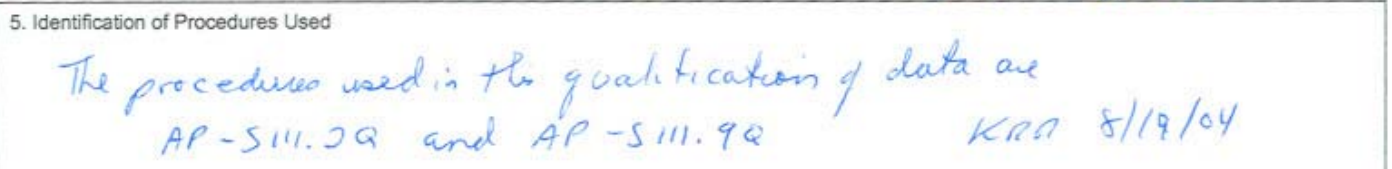 } \\
\hline \multicolumn{3}{|c|}{ Section III. Approval } \\
\hline $\begin{array}{l}\text { Qualification Chairperson Printed Name } \\
\text { Kenneth Rehfeldt }\end{array}$ & $\begin{array}{l}\text { Qualification Chairperson Signaturg } \\
\text { Ten }\end{array}$ & 13,2004 \\
\hline $\begin{array}{l}\text { Responsible Manager Printed Name } \\
\text { Ahmed Monib }\end{array}$ & Responsible Manger Signature - 1 & 13,2004 \\
\hline
\end{tabular}




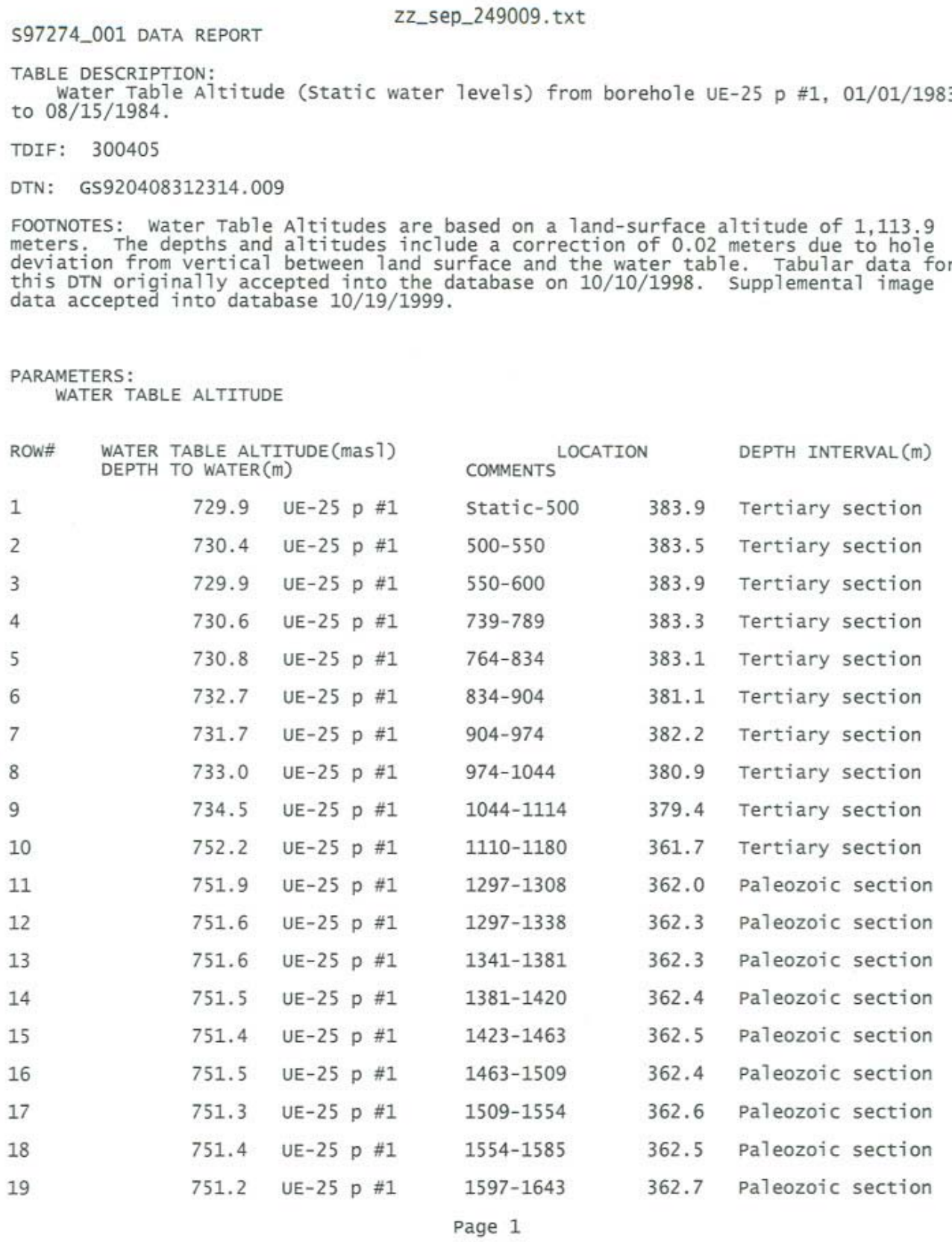


20

21

22

23

$\begin{array}{lll}750.9 & \text { UE-25 p \#1 } & \text { zZ_sep_249009.txt } \\ 751.0 & \text { UE-25 p \#1 } & 1689-1689 \\ 750.8 & \text { UE-25 p \#1 } & 1734-1780 \\ 750.9 & \text { UE-25 p \#1 } & 1780-1805\end{array}$

363.0 Paleozoic section

362.9 Paleozoic section

363.1 Paleozoic section

363.0 Paleozoic section

Page 2 


\begin{tabular}{|l|l|l|}
\hline \multirow{2}{*}{ BSC } & DATA QUALIFICATION PLAN & QA: QA \\
& Page 1 of 1 \\
\hline
\end{tabular}

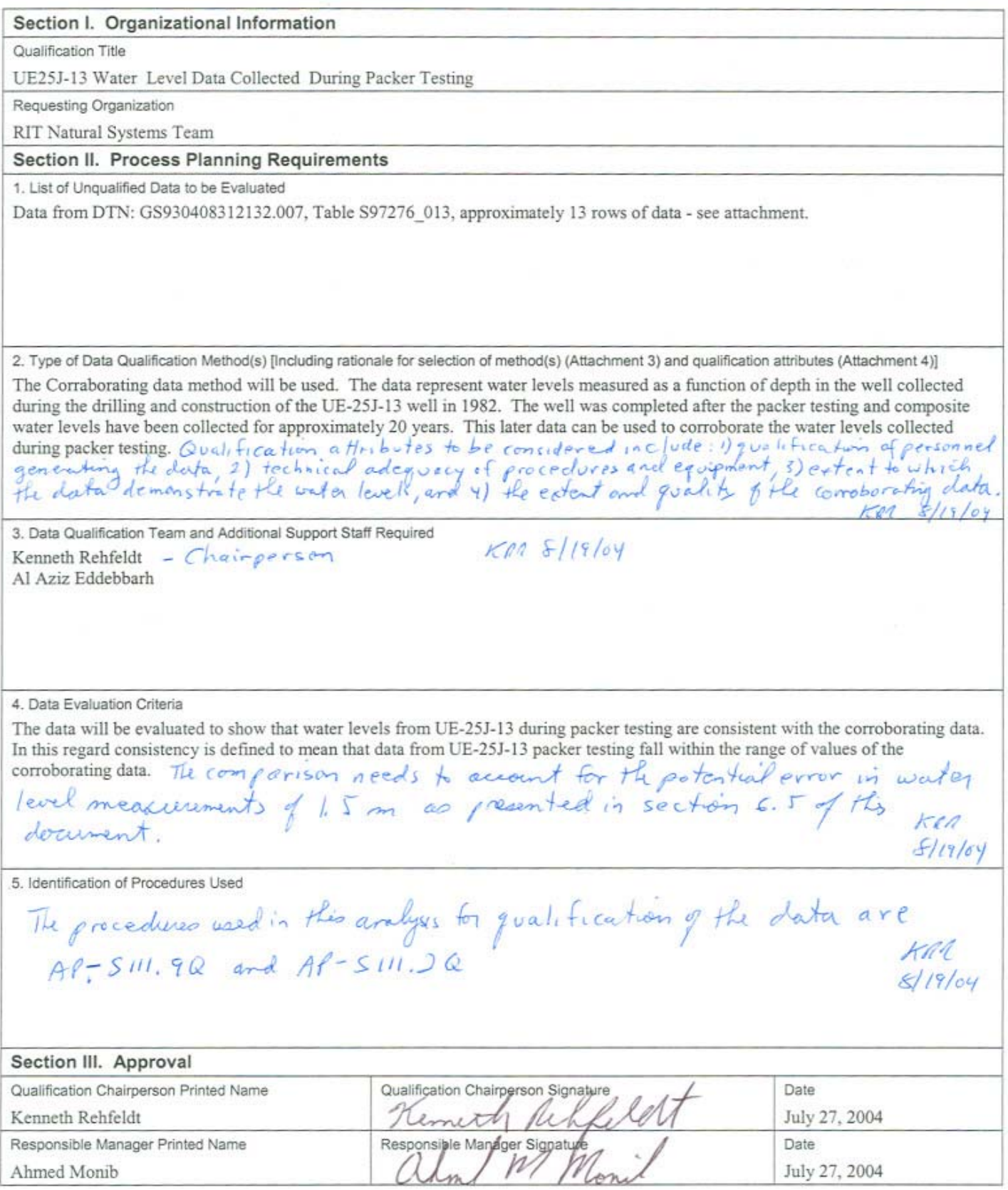


S97276_013 DATA REPORT

zz_sep_247705.txt

TABLE DESCRIPTION:

Depth to water data (static water levels) during hydraulic testing and construction from UE-25 J-13,01/01/1982 to 01/01/1983.

TDIF: 301327

DTN： GS930408312132.007

FOOTNOTES: In the Depth to water column, * indicates nearly recovered to static water leve 1 after 270 minutes. Test is type of test and number. DISCLAIMERS:

Accuracy of static water level values is dependant on the success of certain testing methods. However, accuracy of these methods were not evaluated during collection of the data. Stallman's Method was applied to pumping test values to correct for a highly fractured substrate.

PARAMETERS:

DEPTH TO WATER LITHOSTRATIGRAPHY TEST

LOCATION DEPTH INTERVAL $(m)$

Member

282.2

Member

282.5

$\mathrm{UE}-25 \mathrm{~J}-13$

$282.2-334.1$

Topopah spring

Pumping 1

Member

Pumping 2

4

282.7

UE $-25 \quad J-13$

$282.5-451.1$

Topopah Spring

Calico Hills

Injection 19

5 Calico Hills

Swabbing 19

6282

282.5

UE $-25 \mathrm{~J}-13$

$282.7-451.1$

Topopah Spring

Calico Hil1s

7 282.2 UE-25 J-13 501.1-562.1 Tuffaceous beds of

$\mathrm{Calico} \mathrm{Hills}$ and Prow Pass Member

Swabbing 18

Calico Hills, Prow $282.0 \quad$ UE- 25 J-13 $471.2-612.6$

$\begin{array}{lllll}\text { Swabbing } 2 & 282.4 & \text { UE-25 J-13 } & \text { 371.2-612.6 Tuffaceous beds of }\end{array}$

Calico Hil1s, Prow Pass Member, and tuffaceous sandstone

Swabbing 3

10 282.1 UE-25 J-13 471.2-661.4 Tuffaceous beds of

Calico Hills, Prow Pass Member, tuffaceous sandstone, and Bul1frog Member

Swabbing $6282.4 \quad 584.6-645.6$

11 282.4 UE-25 J-13 584.6-645.6 Prow Pass Member,

15

$283.6+/-2$ * $\quad$ UE -25 J -13

772.7-803.1 Tram unit

Swabbing 11

13

283.3

UE-25 J-13

Page 1

819.9-1063.1 Tram unit, bedded 
tuff, and Tuff of Lithic Ridge zz_sep_249011.txt
Swabbing 20

Page 2 


\section{INTENTIONALLY LEFT BLANK}

